\title{
Versuche an Mauerwerksscheiben unter Normalkraft und Querkraft
}

\section{Working Paper}

Author(s):

Ganz, Hans Rudolf; Thürlimann, Bruno

Publication date:

1984

Permanent link:

https://doi.org/10.3929/ethz-a-000328538

Rights / license:

In Copyright - Non-Commercial Use Permitted

Originally published in:

Bericht / Institut für Baustatik und Konstruktion ETH Zürich 7502(4) 
Versuche an Mauerwerksscheiben unter Normalkraft und Querkraft

Hansruedi Ganz

Bruno Thürlimann 


\section{CIP-Kurztitelaufnahme der Deutschen Bibliothek}

\section{Ganz, Hansruedi:}

Versuche an Mauerwerksscheiben unter Normalkraft und Querkraft / von Hansruedi Ganz; Bruno

Thürlimann. - Basel - Boston - Stuttgart:

Birkhäuser, 1984.

(Bericht/ Institut für Baustatik und Konstruktion,

ETH Zürich; Nr. 7502-4)

ISBN 3-7643-1665-9

NE: Thürlimann, Bruno:; Institut für Baustatik

und Konstruktion <Zürich>: Bericht

Nachdruck verboten.

Alle Rechte, insbesondere das der Übersetzungen in fremde Sprachen und der Reproduktion auf photostatischem Wege oder durch Mikrofilm,

vorbehalten. 


\title{
Versuche an Mauerwerksscheiben \\ unter \\ Normalkraft und Querkraft
}

\author{
von \\ Dipl. Ing. Hansruedi Ganz \\ Prof. Dr. Bruno Thürlimann \\ Institut für Baustatik und Konstruktion \\ Eidgenössische Technische Hochschule Zürich
}




\section{Inhal tsverzeichnis}

1. EINLEITUNG

Seite

1.1 Problemstellung und Zielsetzung

1.2 Versuchsprogramm

2. VERSUCHSKOERPER

2.1 Beschreibung 3

2.2 Baustoffe

2.2.1 Steine

3

2.2.2 Mörtel

3

2.2.3 Bewehrung

3

2.2.3.1 Lagerfugenbewehrung $\quad 3$

$\begin{array}{ll}2.2 .3 .2 \text { Spannstahl } & 4\end{array}$

3. VERSUCHSDURCHFUEHRUNG 25

3.1 Versuchsanlage $\quad 5$

$\begin{array}{ll}3.1 .1 \text { Belastungseinrichtung } & 5\end{array}$

$\begin{array}{ll}3.1 .1 .1 \text { Normalkraft } & 5\end{array}$

3.1.1.2 Querkraft $\quad 5$

$\begin{array}{ll}3.1 .2 \text { Lagerung des Versuchskörpers } & 5\end{array}$

3.2 Versuchsablauf $\quad 6$

3.2.1 Einbau des Versuchskörpers $\quad 6$

$\begin{array}{ll}3.2 .2 \text { Belastung } & 6\end{array}$

$\begin{array}{lr}3.3 \text { Messstellenplan } & 6\end{array}$

4. VERSUCHSRESULTATE $\quad 8$

$\begin{array}{lr}4.1 \text { Auswertung der Versuchsresultate } & 8\end{array}$

$\begin{array}{ll}4.2 \text { Trag- und Bruchverhalten } & 9\end{array}$

4.2.1 Allgemeines $\quad 9$

$\begin{array}{lr}4.2 .2 \text { Versuch } w 1 & 10\end{array}$

4.2.3 Versuch W2 11

4.2 .4 Versuch W3 11

4.2.5 Versuch W4 12

\begin{tabular}{lr}
4.2 .6 Versuch $W 5$ & 12 \\
\hline
\end{tabular}

$\begin{array}{lr}4.2 .7 \text { Versuch } w 6 & 13\end{array}$

4.2.8 Versuch $W 7 \quad 14$

$\begin{array}{ll}4.3 \text { Randverschiebungen } & 15\end{array}$

4.3.1 Horizontale Randverschiebungen $\quad 15$

$\begin{array}{lr}4.3 .2 \text { Vertikale Randverschiebungen } & 15\end{array}$

4.4 Relativverschiebungen zwischen Wand und Betonplatten 16

$\begin{array}{ll}4.5 \text { Verzerrungen } & 16\end{array}$

$\begin{array}{lr}4.5 .1 \text { Dehnungen } \varepsilon_{x} & 17\end{array}$

$\begin{array}{ll}4.5 .2 \text { Dehnungen } \varepsilon_{y} & 17\end{array}$

$\begin{array}{ll}4.5 .3 \text { Schiebungen } \gamma_{x y} & 17\end{array}$

$\begin{array}{lr}4.6 \text { Hauptdehnungen } & 18\end{array}$

$\begin{array}{lr}4.7 \text { Rissverhalten } & 18\end{array}$ 
RESUME

VERDANKUNGEN

BEZEICHNUNGEN

26

TABELLEN

BILDER

31

ANHANG 
1. Elnleltung

\subsection{Problemstellung und Zielsetzung}

Gemauerte Wandscheiben, eingebunden in Stahibetondecken, sind in der Schweiz ein im Hochbau bewährtes und häufig verwendetes Konstruktionsprinzip. Sie verbinden gute bauphysikalische Eigenschaften mit grosser Steifigkeit und Tragfähigkeit unter Vertikal- und Horizontallasten.

Trotz ihres grossen Marktanteils ist das Verhalten von Mauerwerksscheiben unter Horizontallasten wenig erforscht. Der Sicherheitsnachweis beruht auf dem Vergleich von elastisch gerechneten Spannungen mit normierten zulässigen Spannungen. Die zulässigen Spannungen basieren auf empirischen Formeln. Es fehlt also ein physikalisches Tragmode11, das die wesentlichen Einflusse auf das Tragverhalten der Mauerwerksscheiben richtig erfasst.

Ziel des Forschungsprojektes "Trag- und Bruchverhalten von Mauerwerksscheiben" ist das Bereitstellen von Grundlagen für die rechnerische Erfassung des Verhaltens von Scheiben unter Normalkraft, Biegung und Querkraft in der Wandebene. Dazu wurden im ersten Teil des Forschungsprojektes Versuche am Mauerwerks-Element, sogenannte "Kleinkörper-Versuche" [1], durchgeführt. Ziel dieser Versuche war die Ermittlung der Bruchbedingung und des Stoffgesetzes von Mauerwerk unter einem beliebigen zweiachsigen Spannungszustand. Ziel des zweiten Teils des Forschungsprojektes ist die Ermittlung des Tragverhaltens von Mauerwerksscheiben. Aufgrund dieser "Wand-Versuche" soll ein theoretisches Tragmodell überprift und verbessert werden. Werden Bruchbedingungen und Stoffgesetze in dieses Tragmodell eingesetzt, lassen sich Bruchlast und Verformungen der Mauerwerksscheibe berechnen.

Das Forschungsprojekt sol1 Grundlagen für ein neues Verfahren bereitstellen, das eine sichere und wirtschaftliche Bemessung von Mauerwerksscheiben auf die Grenzzustände der Tragfähigkeit und der Gebrauchsfähigkeit erlaubt.

\subsection{Versuchsprogramm}

Das experimentelle Programm zur Ermittlung des Tragverhaltens von Mauerwerksscheiben ist in Tabelle 1 zusammengestellt. Die Bilder 7 und 8 zeigen ein Schema der Versuchsanlage.

Die Mauerwerksscheiben waren zwischen zwei Betonplatten gelagert. Die untere Platte war auf den Aufspannboden fixiert. An ihrem oberen Ende wurden die Wände durch eine Normalkraft mit vorgegebener Exzentrizität belastet. Anschliessend wurde das obere Ende durch eine Querkraft (Schub) belastet und die Horizontalverschiebung stufenweise bis zum Bruch der Wände gesteigert.

Folgende Parameter wurden variiert:

\section{- Normalkraft:}

Es wurden zwei Lastniveaus untersucht. Die kleinere bzw. grössere Normalkraft entspricht der Last im untersten Geschoss eines ca. vierstöckigen resp. ca. zehnstöckigen Gebäudes (vgl. [3]).

\section{- Exzentrizität der Normalkraft in der Wandebene:}

Nebst gleichmässig verteilter Normalkraft (Exzentrizität $e=0$ ) wurde der Einfluss von zusätzlicher Biegung auf die Schubtragfähigkeit der Wände untersucht.

\section{- Bewehrung:}

In der Schweiz werden vermehrt Bewehrungen in die Lagerfugen eingelegt. Deshalb wurde in zwei Versuchen der Einfluss dieser "Bügel" auf das Verhalten der Wände untersucht.

Da die Geometrie der in der Schweiz gebräuchlichen Backsteine das Einlegen einer schlaffen Vertikalbewehrung (senkrecht zu den Lagerfugen) praktisch kaum ermöglicht, wurde statt dessen in einem Versuch 
eine vertikale Vorspannung ohne Verbund (ausserhalb der Wand) aufgebracht.

\section{- Schub-Belastung:}

In den ersten fünf Versuchen wurde die Horizontallast progressiv gesteigert. An zwei weiteren Wandscheiben wurde untersucht, welchen Einfluss eine Aenderung der Belastungsrichtung auf das Verhalten der Wände hat. Mit diesen zyklischen Versuchen wurde die Wirkung von Wind oder Erdbeben auf Mauerwerksscheiben simuliert.

In den Versuchen wurden folgende Grössen beobachtet:

- Querkraft (Schub) und Normalkraft

- Horizontale Verschiebung des oberen Wandendes

- Horizontale und vertikale Randverschiebungen der Wände

- Relativverschiebung zwischen Wand und Betonplatten

- Knotenverschiebungen auf den Mauerwerkswänden

- Rissbild und Rissweiten

In allen Versuchen wurde eine einzige Steinsorte und ein Zementmörtel verwendet. 


\section{Versuchskörper}

\subsection{Beschreibung}

Der Versuchskörper ist im Bild 1 dargestellt. Um den Messaufwand etwas zu reduzieren, wurde die Höhe der Wände im Vergleich zur normalen Stockwerkshöhe auf ca. $80 \%$ verkleinert.

Die Wände wurden von einem Maurer des Prüf- und Forschungsinstitutes des Verbandes der Schweizerischen Ziegel- und Steinfabrikanten erste11t.

Die Scheiben waren $3.6 \mathrm{~m}$ lang und $2 \mathrm{~m}$ hoch. An beiden Wandenden waren sie durch einen $\mathrm{Flansch}$ von $0.6 \mathrm{~m}$ Breite seitlich stabilisiert. In den zwei Versuchen mit exzentrischer Normalkraft wurden die Flanschen auf $0.9 \mathrm{~m}$ Breite verstärkt. Die Mauerdicke betrug durchwegs $150 \mathrm{~mm}$.

Die Mauerwerkswände standen auf einer $180 \mathrm{~mm}$ dicken Betonplatte, die auf dem Aufspannboden lag. Auf das obere Wandende wurde mit einer Mörtelfuge eine in Längsrichtung auf $600 \mathrm{kN}$ vorgespannte Platte von $160 \mathrm{~mm}$ Dicke aufgelegt.

Die Lagerfugen hatten eine mittlere Dicke von $10 \mathrm{~mm}$ und waren vollflächig vermörtelt. In den Stossfugen wurde der Mörtel nur als verdeckter Spatz in die Steinnuten der Modulsteine eingebracht. Dadurch hatten die Aussenschalen der Backsteine keinen Kontakt in den Stossfugen.

In die Versuchskörper W3 und W5 wurden in jede Lagerfuge zwei Bewehrungsstäbe $\emptyset 5 \mathrm{~mm}$ eingelegt und ausserhalb der Wand mit Stahlplatten $80 \times 150 \times 8 \mathrm{~mm}$ verankert. Die Flanschen waren in ihrer Ebene nicht bewehrt. Zusätzlich wurde der Versuchskörper W5 mit zwei Spannstählen $\emptyset 12 \mathrm{~mm}$ vertikal vorgespannt. Die Lage der Spannstähle ist im Bild 1 festgehalten.

\subsection{Baustoffe}

Backstein und Mörtel wurden den Normprüfungen unterzogen. Die Normprüfungen sind in der Norm SIA 177 [2] beschrieben.

\subsubsection{Steine}

Es wurde eine einzige Steinsorte, ein Modulbackstein, verwendet. Bild 2 zeigt das Lochbild dieses Steines. In der Tabelle 2 sind die Resultate der Normprüfungen aufgeführt. Da der gleiche Stein, wie er in den Kleinkörper-Versuchen [1] verwendet worden war, nicht mehr zur Verfügung stand, wurde ein neuer Stein verwendet. Materialkennwerte und Lochbild der beiden Provenienzen unterschieden sich nur geringfügig.

\subsubsection{Mörtel}

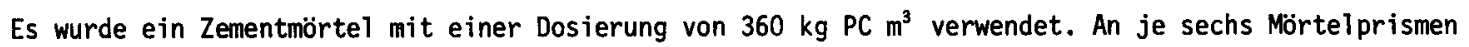
$40 \times 40 \times 160 \mathrm{~mm}$ wurden die Biegezugfestigkeit und die Würfeldruckfestigkeit bestimmt und gemittelt. In der Tabelle 3 sind die Prüfwerte zusammengestellt. Bild 3 zeigt die Siebkurven der verwendeten Sande.

\subsubsection{Bewehrung}

\subsubsection{Lagerfugenbewehrung}

In den Versuchen $W 3$ und W5 wurden in jede Lagerfuge zwei kaltverformte, gerippte Stäbe $\emptyset \mathrm{mm}$ eingelegt. Auf dem Markt werden diese Stäbe mit einer eingeschweissten Diagonale angeboten (Murfor). Die Verankerung der Bewehrung an den Wandenden wurde mit Ankerplatten gewährleistet.

Die Materialkennwerte der Bewehrung sind in der Tabelle 4 zusammengestellt. Die Festigkeitswerte wurden verformungsgesteuert mit einer Dehnungsgeschwindigkeit gemäss Norm SIA 162 , RL 9, von $\dot{\varepsilon}=0.3 \cdot 10^{-2} / \mathrm{min}$ [4] bestimmt. Die statischen Festigkeitswerte wurden nach einem zweiminütigen Konstanthalten der Dehnung abge- 
lesen. Die freie Probenlänge betrug $960 \mathrm{~mm}$. Die Stahldehnung $\varepsilon_{s}$ wurde aus dem Kolbenhub der Prüfmaschine und der freien Probenlänge berechnet. Bild 4 zeigt das aus einem Zugversuch gewonnene Spannungs-DehnungsDiagramm.

Die Stabquerschnittsfläche $A_{\text {eff }}$ wurde aus einer Gewichtsmessung bestimmt.

\subsubsection{Spannstahl}

Die Wand W5 war mit zwei Spannstählen $12 \mathrm{~mm}$ vertikal vorgespannt. Die dynamischen Festigkeitswerte des Vorspannstahls sind ebenfalls in der Tabelle 4 zusammengestellt. Laut Angaben des Herstellers wurden diese Werte an je zwei Proben aus acht Ringen (insgesamt 16 Proben) ermittelt. 


\section{Versuchsdurchfuhrung}

Die Versuche wurden auf dem Aufspannboden der Forschungshalle der ETH-Hönggerberg in Zürich durchgeführt.

\subsection{Versuchsanlage}

In der speziell für diese Versuche entworfenen Versuchsanlage konnten die Wände durch Normalkraft und Querkraft belastet werden. Die Resultierende der Normalkraft wirkte zentrisch oder exzentrisch zur Scheibenachse. Die Richtung der Schubbelastung war umkehrbar. Die Bilder 5 und 6 zeigen je eine Gesamtansicht der Anlage für zentrische bzw. exzentrische Normalkraft.

\subsubsection{Belastungseinrichtung}

Die Versuchsanlage ist schematisch in den Bildern 7 und 8 dargestellt.

\subsubsection{Norma lkraft}

Die zentrische Normalkraft wurde von je sechs vertikal montierten $135 \mathrm{kN}$-Zugkolben beidseits der Wand an die obere Betonplatte abgegeben. Die Kolben waren über ein Stahlprofil auf den Aufspannboden verankert. Alle Zylinder waren an einen Ausgang eines Pendelmanometers angeschlossen. Ihre Verteilung entlang der Scheibe war so gewählt, dass jeder Kolben auf eine gleich grosse Wandfläche wirkte. In den Versuchen mit hoher Normalkraft (W2, W7) musste die obere Betonplatte mit Stahlprofilen verstärkt werden.

Die exzentrische Normalkraft wurde von je einem $400 \mathrm{kN}$-Zugkolben beidseits der Wand an einen Stahlquerträger abgegeben. Der Querträger lag auf einem Stahlkastenträger, der die Betonplatte in Längsrichtung versteifte und damit die Last über die Wand verteilte. Die Lagerung der Kolben und der Anschluss an das Pendelmanometer waren gleich gelöst wie bei zentrischer Normalkraft.

\subsubsection{Querkraft}

Der Schub wurde von einem servohydraulisch gesteuerten $Z y l$ inder $\left(F_{\max }= \pm 1000 \mathrm{kN}\right)$ aufgebracht. Der $Z y l i n-$ der war gelenkig an einen Stahlrahmen montiert. Abspannungen mit Stahlseilen hielten den Zylinder in horizontaler Lage und übernahmen sein Eigengewicht. In den statischen Versuchen wurde die Querkraft als Zugkraft über eine Stahlplatte auf die vorgespannten Stahlstangen $26 \mathrm{~mm}$ in der oberen Betonplatte abgegeben. Bei umgekehrter Lastrichtung (zyklische Versuche) wurde eine Druckkraft direkt auf die Stirnseite der Betonplatte ausgeübt.

\subsubsection{Lagerung des Versuchskörpers}

Die untere Betonplatte wurde mit acht Stahlstäben auf den Aufspannboden gespannt. Dadurch wurde ein Abheben der Platte vom Boden verhindert. Um ein gleichmässiges Aufliegen auf dem Boden zu gewährleisten, wurde unter die Betonplatte eine $5 \mathrm{~mm}$ dicke Hartfaserplatte eingelegt. Wie vor allem im Versuch W2 festgestelit werden konnte, wurden die Unebenheiten der Betonplatte durch die Hartfaserplatte zu wenig ausgeglichen: Ab Versuch W3 wurde deshalb zwischen Betonplatte und Aufspannboden eine Gipsausgleichsschicht eingebracht. Die Betonplatte war an beiden Enden mit Stahlwiderlager gegen Gleiten gesichert.

An der oberen Betonplatte waren Stahlstangen zur Stabilisierung der Platte angebracht. Mit zwei (in den zyklischen Versuchen vier) horizontalen Stangen konnte ein seitliches Ausweichen in Plattenebene verhindert werden. Mit zwei vertikalen Stangen konnte die Platte parallel zum Boden gehalten werden. Die vertikalen Stangen brauchten nie, die horizontalen nur sehr selten eingesetzt zu werden. 


\subsubsection{Einbau des Versuchskörpers}

Die Wände wurden auf der unteren Betonplatte in der Versuchsanlage erstellt. Um einen guten Verbund zwischen Betonplatte und Wand zu gewährleisten, wurden die Betonplatten mit einem Meissel aufgerauht. Nach ca. zwei Tagen wurde die obere, in Längsrichtung vorgespannte Betonplatte mit Zementmörtel auf die Wand versetzt. Wegen der Saugfähigkeit der Backsteine wurde dem Mörtel das Wasser sehr schnell entzogen. Dies ergab einige Probleme beim Versetzen der Platte beim Versuchskörper W1. Ab Versuch W2 wurde dem Mörtel ein Zusatzmittel (Methocel) beigemengt, das die Wasserabgabe verringerte. Danach wurden der Horizontalkolben (Querkraft) und die Vertikalkolben (Normalkraft) angeschlossen und zentriert.

Um die Rissentwicklung möglichst gut verfolgen zu können, wurden die Wände weiss gestrichen.

\subsubsection{Belastung}

Das Alter der Wände bei Prüfbeginn lag zwischen 16 und 22 Tagen. Die Versuche dauerten 3 bis 6 Tage. Alle Versuche wurden verformungsgesteuert durchgeführt. Steuergrösse war die Horizontalverschiebung der oberen Betonplatte. Während der Messungen wurde die Horizontalverschiebung konstant gehalten. Die dabei auftretende Relaxation zeigte sich im Abfallen der Querkraft vom Anfangswert $V_{A}$ auf den Endwert $V_{E}$. Bei allen Versuchen wurde die Normalkraft zuerst aufgebracht und danach während der ganzen Versuchsdauer konstant gehaiten.

In den Versuchen mit progressiver Belastung wurde die Horizontalverschiebung (in Richtung der Querkraft) der oberen Betonplatte stufenweise gesteigert bis zum Bruch der Wand. Die Querkraft hatte während des ganzen Versuchs das gleiche Vorzeichen.

Die zyklische Beanspruchung der Versuchskörper w6 und W7 wurde ebenfalls statisch (keine Trägheitskräfte) aufgebracht. Unter zyklischer Beanspruchung wird in diesem Versuchsbericht eine alternierende Beanspruchung mit Vorzeichenwechsel der Querkraft verstanden. Mit zwei bis drei Zwischenablesungen wurde die gewünschte Maximalverschiebung $+\delta_{h, i}$ der Laststufe LSi aufgebracht. Genau gleich wie in den Versuchen mit progressiver Belastung wurde nun eine volle Ablesung vorgenommen. Nach zehn Zyklen mit Zwischenablesungen be $i+\delta_{h, j}$ und $-\delta_{h, i}$ wurde bei $+\delta_{h, i}$ wieder eine volle Ablesung gemacht. Anschliessend wurde eine neue Maximalverschiebung $+\delta_{h, i+1}$ der LS $i+1$ aufgebracht und der gesamte Vorgang wiederholt.

Die Zwischenablesungen wurden nach 30 Sekunden Konstanthalten der Verschiebung $\delta_{h}$ durchgeführt, vor einer vollen Ablesung nach 30 Sekunden und nach zwei Minuten. Der zeitliche Ablauf einer Laststufe in einem Versuch mit progressiver bzw. zyklischer Belastung ist im Bild 9 dargestellt.

\subsection{Messstellenplan}

Folgende Messungen wurden durchgeführt:

\section{a) Normal kraft}

- Messung der Normalkräfte an jedem Kolben mittels $200 \mathrm{kN}$ bzw. $300 \mathrm{kN}$ Kraftmessdosen.

- Messung des 0eldruckes am Pendelmanometer mittels einer 500 bar Flüssigkeitsdruckmessdose.

- Lastablesung am Pendelmanometer.

\section{b) Querkraft}

- Messung der Querkraft mittels zwei $300 \mathrm{kN}$ oder $500 \mathrm{kN}$ Kraftmessdosen (Zug) und vier $300 \mathrm{kN}$ Kraftmessdosen (Druck). In den zyklischen Versuchen wurde zwischen den Kraftmessdosen auf Zug und auf Druck ein Spalt belassen, damit die Messdosen eine eindeutige Nullstellung behielten. 
- Messung des Differenzdruckes in den Kammern des servohydraulischen Zylinders mittels zweier 200 bar Flüssigkeitsdruckmessdosen.

\section{c) Horizontalverschiebung der oberen Betonplatte}

- Messung der Verschiebung an beiden Plattenenden mit insgesamt drei induktiven Wegaufnehmern (Genauigkeit $2 \cdot 10^{-3} \mathrm{~mm}$ ).

- Messung des Kolbenweges.

d) Horizontalverschiebung der unteren Betonplatte

- Messung der Verschiebung mit einem induktiven Wegaufnehmer (Genauigkeit $1 \cdot 10^{-3} \mathrm{~mm}$ ).

e) Scheibenrandverschiebungen

- Messungen der horizontalen Verschiebungen der seitlichen Ränder gegen einen Messrahmen mit einer induktiven Messstange (Genauigkeit $5 \cdot 10^{-3} \mathrm{~mm}$ ).

- Messung der vertikalen Verschiebungen der Wand zwischen den Betonplatten mit einer induktiven Messstange auf beiden Seiten der Wand (Genauigkeit $1 \cdot 10^{-2} \mathrm{~mm}$ ).

\section{f) Knotenverschiebungen}

- Messung der Verschiebungen auf der Wandvorder- und -rückseite sowie auf den Aussenseiten der Flanschen in einem Messnetz. Auf der Rückseite der Wand war nur ein Ausschnitt des vollen Netzes der Vorderseite angeordnet.

Die Relativverschiebungen zwischen Mauerwerkswand und Betonplatten konnten ebenfalls gemessen werden. Dazu wurden Winkelprofile auf die Betonplatten geklebt und an das Messnetz angeschlossen.

Die Verschiebungen wurden mit induktiven Deformetern der Basislängen 150, 200, 250,300, 400 und $500 \mathrm{~mm}$ gemessen. Die Genauigkeit beträgt beim $400 \mathrm{~mm}$ Gerät $2 \cdot 10^{-3} \mathrm{~mm}$, bei allen anderen Geräten $1 \cdot 10^{-3} \mathrm{~mm}$. Die Deformeter wurden auf Bolzen aufgesetzt, die auf die Backsteine geklebt waren.

\section{g) Neigung der oberen Betonplatte}

- Messung der Verdrehung der oberen Betonplatte um die Wandebene mit einem Klinometer (Genauigkeit $2 \cdot 10^{-3}$ Altgrad). Diese Messung steuerte den Einsatz der horizontalen Stabilisierungsstangen.

\section{h) Seitliche Auslenkung der oberen Betonplatte}

- Messung der horizontalen Auslenkung in der Ebene der oberen Betonplatte mit einer Messuhr (Genauigkeit $\left.1 \cdot 10^{-2} \mathrm{~mm}\right)$. Diese Messung steuerte den Einsatz der horizontalen Stabilisierungsstangen.

\section{i) Risse}

Nach jeder Laststufe wurden die Risse mit schwarzem Filzschreiber auf der Wand nachgezeichnet. Die Weiten einer repräsentativen Auswahl von Rissen wurden mit einem Rissmikroskop (Abschätzung auf $1 \cdot 10^{-2} \mathrm{~mm}$ ), ab Rissweiten von ca. $0.5 \mathrm{~mm}$ mit einem Rissmassstab (Abschätzung auf $5 \cdot 10^{-2} \mathrm{~mm}$ ), bestimmt. Die Rissweiten wurden in einem Protokoll und auf der Wand festgehalten. Anschliessend wurde die Wand fotografiert.

In den zyklischen Versuchen wurden bei jeder vollen Ablesung (d.h. zweimal pro LS) Rissbild und Rissweiten nachgetragen.

In den Zwischenablesungen wurden nur die Messungen a) bis d), g) und h) durchgeführt.

Die Lage der Geräte für die Messungen a), b), g) und h) ist in den Bildern 7 und 8 eingetragen. Die Anordnung der restlichen Messstellen ist im Bild 10 dargestellt. 
4. Versuchsresul tate

\subsection{Auswertung der Versuchsresul tate}

\section{a) Normalkraft}

Die in diesem Bericht angegebenen Normalkräfte setzen sich aus den Kolbenlasten und aus dem rechnerischen Gewicht der oberen Betonplatte und allfälliger Stahlaufbauten zusammen. Sie beziehen sich auf einen Schnitt an der Unterkante der oberen Betonplatte.

Eine Korrektur der Normalkraft infolge Schiefstellung der Zugkolben wurde nicht vorgenommen, da der Kosinus der auftretenden Winkel stets grösser als 0.9995 war ( $\mathrm{vgl}$. auch b) Querkraft).

\section{b) Querkraft}

Infolge von Horizontalverschiebung der oberen Betonplatte stellten sich die Zugkolben für die Normalkraft schief. Dadurch wurde eine der aufgebrachten Querkraft entgegenwirkende Komponente erzeugt. Die im Bericht angegebenen Querkräfte $V$ wurden nach folgender Beziehung berechnet:

$$
V=v_{e x}-\left|N_{e x}\right| \cdot \frac{\delta_{h}}{H}
$$

Die Bedeutung der Grössen $V_{e x}, N_{e x}, \delta_{h}$ und $H$ ist aus Bild 11 ersichtlich.

\section{c) Horizontal verschiebung}

Die im Bericht angegebenen Horizontalverschiebungen $\delta_{h}$ wurden als Differenzen der Messstellen 22 und 38 (bzw. 22 und 23 für die Versuche $W 6$ und W7) berechnet. Eine allfällige Starrkörperverschiebung der Wand konnte so eliminiert werden. Die Messstellen 22, 23 und 38 sind im Bild 10 dargestellt.

\section{d) Randverschiebungen}

Die horizontalen Randverschiebungen wurden als Differenzen zur Messstelle 38 (bzw. 23 für die Versuche W6 und W7) bestimmt (vgl, auch c)).

Als vertikale Randverschiebungen wurden die Mittelwerte der Messstellen 49 bis 52 und 54 bis 57 dargestellt (vgl. Bild 10).

\section{e; Relativverschiebung zwischen Wand und Betonplatte}

Die horizontalen Relativverschiebungen wurden als Mittelwerte aus je zwei Messstrecken bestimmt.

Durch das Ausmessen von zwei horizontalen Verschiebungen pro Anschlusspunkt Betonplatte - Messnetz konnte der Einfluss der vertikalen auf die horizontale Verschiebung rechnerisch eliminiert werden.

\section{f) Knotenverschiebungen}

Durch die Ausmessung des Messnetzes (Bild 10) wurden die Knotenverschiebungen überbestimmt. Um den notwendigen Ausgleich der Messungen zu erreichen, wurde das Messnetz auf der Mauerwerksscheibe als Fachwerk aufgefasst. Die aus den Messungen gerechneten Dehnungen wurden als Temperaturdehnungen der Stäbe betrachtet. Mit diesen Zwängungen wurden das statisch unbestimmte Fachwerk belastet und die Verschiebungen der Messnetzknoten berechnet. Die Steifigkeit der Fachwerkstäbe, E•A/1, wurde konstant angesetzt.

\section{g) Verzerrungen}

Die Dehnungen und Schiebungen wurden aus den Knotenverschiebungen berechnet. Als Richtungen des Koordinatensystems wurden die Fugenrichtungen gewählt. 
Die Knoten des Messnetzes wurden als Eckpunkte rechteckiger Elemente mit bekannten Verschiebungen, den Knotenverschiebungen, aufgefasst. Aus den Eckpunktverschiebungen wurden dann mittlere, über das Element konstante, Verzerrungen berechnet. Die Elementbreite (parallel zur Lagerfuge) betrug durchwegs eine Steinund Fugenbreite, die Elementhöhe (senkrecht zur Lagerfuge) eine bzw. zwei Stein- und Fugenhöhen (vgl. Bild 10).

Die Geometrie und die verwendeten Formeln sind im Bild 12 dargestellt.

\section{h) Risse}

Es wurden in allen Bereichen der Wandscheiben Risse gemessen und ihre Entwicklung während des Versuchs verfolgt. Ihre Grösse entsprach mittleren bis maximalen Rissweiten in diesen Bereichen. Im Bericht sind sowohl die auf der Wand maximal gemessenen Rissweiten als auch die Entwicklung einer Auswahl von Rissen dargeste11t.

Es wurde in "Risse in Steinen" und in "Risse in Lagerfugen" unterteilt. Wegen der besonderen Ausbildung der Stossfugen (Mörtel nur als verdeckter Spatz in der Steinnut) konnten dort keine Rissweiten bestimmt werden.

\subsection{Trag- und Bruchverhalten}

\subsubsection{Allgemeines}

Das generelle Tragverhalten der Mauerwerkswände ist in den Bildern 13 bis 23, Querkraft-Horizontalverschiebung, wiedergegeben. Eine tabellarische Zusammenstellung der wichtigsten Messgrössen mit der Bezeichnung der Laststufen ist im Anhang gegeben.

In den Abbildungen 27, 28 und 78, 79 sind die Bruchbilder bzw. Rissbilder der Versuchskörper festgehalten.

Die Kurven "Querkraft-Horizontalverschiebung" lassen sich für alle Versuche in drei Abschnitte aufteilen.

- Im elastischen Bereich nehmen die Verschiebungen proportional zur Querkraft zu. Die Wände sind ungerissen und die, allerdings kleine, Zugfestigkeit des Mauerwerks ist noch voll wirksam. Die elastische Phase reichte in allen Versuchen bis ca. zur halben Maximallast $v_{E, u}$. Wegen der grossen Steifigkeit der Wände lag die zugehörige Verschiebung unter $1.0 \mathrm{~mm}$.

- Ab ca. der halben Maximallast setzt die Rissbildung ein. Die Verschiebungen nehmen nun stärker zu und die Last-Verschiebungs-Kurven krümmen sich. Mit einsetzender Rissbildung wird die Zugfestigkeit des Mauerwerks sukzessive abgebaut. Parallel zu diesem Abbau lagern sich die Spannungen in den Wänden um. Kurz vor oder gleichzeitig mit dem Erreichen der Maximallast treten die ersten Risse im Schnitt von Druckflansch und Wand auf. Sie verlaufen vertikal und spalten die Wände in ihrer Ebene vertikal auf. Der erste Riss im Druckflansch trat immer in der -zweiten Steinlage von unten auf. Damit ist die Umlagerung der Spannungen abgeschlossen und der maximale Widerstand $V_{E, u}$ der Wand ist mobilisiert.

- Wird die Horizontal-Verschiebung weiter gesteigert, so fällt die Querkraft ab. Die Neigung der LastVerschiebungs-Kurve hängt vor allem von der Höhe der Normalkraft ab. Die Steine in der Druckecke der Wand werden nach und nach zerstört und der Oruckflansch spaltet sich in seiner Ebene auf. Damit wird der Bruch der Wand eingeleitet.

Die Höhe der Normalkraft hat den grössten Einfluss auf das Tragverhalten der Wände. Bei kleiner Normalkraft verhalten sich die. Wände recht duktil. Der Lastabfall nach Ueberschreiten der Maximallast ist gering. Wird die Normalkraft gesteigert, so wächst auch die maximal erreichbare Querkraft an. Dagegen sinkt die Duktilität der Versuchskörper. Die Verschiebungen bis zum Erreichen des Bruchs nehmen stark ab. 
Dagegen hat die Normalkraft keinen Einfluss auf das Verhalten (Steifigkeit) der Wände im elastischen Bereich.

Die Flanschen an den Enden der Wände erwiesen sich als sehr günstig für die Versuchsdurchführung. Kleine, unvermeidbare Exzentrizitäten der Normalkraft aus der Wandebene wurden durch die Flanschen aufgenommen. Dadurch wurde eine zentrische Beanspruchung der Wände erreicht. Weiterhin lässt sich vermuten, dass die Flanschen das Aufreissen der Steine senkrecht zur Wandebene im Druckbereich etwas behinderten und so ein duktileres Verhalten der Wände bewirkten.

Die Bewehrung in den Versuchskörpern $W 3$ und W5 hatten unterschiedliche Folgen. Eine Anordnung von Lagerfugen-Bewehrung allein, wie im Versuch $W 3$, bewirkte nur eine geringe Erhöhung der maximalen Querkraft. Dagegen war die maximale Verschiebung ca. dreimal so gross wie im unbewehrten Versuch W1. Die maximale Querkraft konnte über weite Bereiche gehalten werden. Jedoch muss hier auf die gute Verankerung der Bügel aufmerksam gemacht werden. Ohne die Anwendung von Ankerplatten wären wohl kaum die gleich guten Resultate erzielt worden.

Die vertikale Vorspannung im Versuch W5 ermöglichte eine starke Zunahme der maximalen Querkraft. Da das Versagen von Wänden mit kleiner exzentrischer Normalkraft eher als Biegebruch denn als Schubbruch bezeichnet werden muss, wirkt sich die Erhöhung des Biegewiderstandes mit einer Bewehrung sehr günstig aus.

Bei gegebener Normalkraft bewirkt ein zusätzliches Biegemoment in Richtung der Querkraft eine Reduktion der maximal möglichen Schubkraft.

Die zyklische Belastung der Mauerwerkswände, wie sie durch Wind und Erdbeben entstehen, hatte bis zu einer Höhe der Querkraft von ca. der Hälfte der statischen Traglast keinen Einfluss auf das Verhalten der Wände. Die Abminderung der Traglast infolge zyklischer Belastung im Vergleich zur progressiven Belastung lag bei ca. $15 \%$. Dagegen wurde die Bruchverformung in den zyklischen Versuchen stark reduziert.

\subsubsection{Versuch w1}

Der Verlauf von Querkraft und Horizontalverschiebung im Versuch $W 1$ ist im Bild 13 dargestellt. Die zugehörigen Messwerte sind in der Tabelle Al zusammengestellt.

Die Normalkraft betrug in diesem Versuch $-415 \mathrm{kN}$ und war gleichmässig verteilt. Die Wand war unbewehrt.

Die elastische Phase reichte bei W1 bis zu 50\% der Bruchlast, bei einer Horizontalverschiebung von ca. $0.8 \mathrm{~mm}$. Auf dieser Lasthöhe waren nur ganz wenige Anrisse zu sehen. Sie traten in den Lagerfugen des vorderen Wandteils (Seite Flansch R, Bild 10) auf und hatten eine maximale Rissweite von $0.05 \mathrm{~mm}$. Kurz danach erschienen die ersten Risse im Zugflansch, und die Risse in der Wand verlagerten sich nach hinten (Seite Flansch L, Bild 10). Bei 73\% der Bruchlast war der Zugflansch in der zweiten Mörtelfuge über der unteren Betonplatte durchgehend gerissen. Parallel zu diesem Abheben auf der Zugseite konzentrierten sich die Druckspannungen auf der gegenüberliegenden Wandseite. Dies führte bei ca. $85 \%$ der Bruchlast zu ersten Rissen in Steinen. Bei 95\% der Bruchlast trat der erste Riss im Druckflansch auf. Bei einer Horizontalverschiebung von ca. $8 \mathrm{~mm}$ war die maximale Querkraft, die Bruchlast, erreicht. Der grösste Riss lag im Zugflansch, seine Weite betrug $3 \mathrm{~mm}$. Im Bild 78 ist die Rissverteilung in diesem Stadium des Versuchs festgehalten. Mit zunehmender Horizontalverschiebung fiel die Querkraft leicht, aber konstant ab. Bei $16 \mathrm{~mm}$, der doppelten Verschiebung bei Maximallast, betrug die Querkraft noch ca. 85\% der Bruchlast. Bei ca. $17 \mathrm{~mm}$ Horizontalverschiebung spaltete sich der Druckflansch in seiner Ebene auf. Der totale Verlust an Tragfähigkeit erfolgte beim Herausstossen der Druckdiagonalen aus dem Druckflansch. Dabei wurde auch der unterste Stein (direkt auf der Betonplatte) zerstört, und die Wand schob entlang einer geneigten Linie ab. Entlang dieser Linie und in der Druckecke der Wand waren die Steine zerstört. Die Horizontalverschiebung betrug in dieser Phase ca. $28 \mathrm{~mm}$, und die Wand trug kurz zuvor noch eine Querkraft von ungefähr $120 \mathrm{kN}$. Das Bruchbild von W1 ist im Bild 27 dargestellt. 


\subsubsection{Versuch w2}

Der Verlauf von Querkraft und Horizontalverschiebung im Versuch W2 ist im Bild 14 festgehalten. Die zugehörigen Messwerte sind in der Tabelle A2 zusammengestellt. Die gleichmässig verteilte Normalkraft betrug in diesem Versuch $-1287 \mathrm{kN}$. Die Wand war unbewehrt.

Wie bereits früher bemerkt wurde, genügte die Hartfaserplatte zwischen der unteren Betonplatte und dem Aufspannboden nicht, um alle Unebenheiten auszugleichen. Wie die Auswertung des Deformeter-Messnetzes zeigte, lag die Betonplatte in der Mitte der Wand nicht satt auf dem Boden auf. Der darüberstehende Teil der Wand entzog sich deshalb der Last, und die Wandenden wurden stärker beansprucht. Die ungleichmässige Lastverteilung machte sich in starkem Masse in der elastischen Phase des Versuches bemerkbar (siehe Bilder 53 bis 55 und 72). So entstanden bereits unter Normalkraft allein mehrere Risse, und die Steifigkeit der Wand war kleiner als in den anderen Versuchen. Durch unelastische Verformungen wurde die ungleichmässige Lastverteilung im Verlaufe des Versuches zum Teil korrigiert. Doch scheint sie, verglichen mit dem Versuch W7, trotzdem einen Einfluss auf die Bruchlast gehabt zu haben. Die Abminderung der Bruchlast infolge der ungieichmässigen Lagerung dürfte zwischen 10 bis $15 \%$ gelegen haben.

Unter Normalkraft allein betrug die maximale Rissweite bereits $0.12 \mathrm{~mm}$. Sie wuchs bis zur halben Bruchlast auf $0.45 \mathrm{~mm}$. Bei gleicher Querkraft betrug die maximale Rissweite im Versuch W7 dagegen nur $0.10 \mathrm{~mm}$. Die Horizontalverschiebung betrug in dieser Laststufe im Versuch $W 21.4 \mathrm{~mm}$, im Versuch W7 $0.85 \mathrm{~mm}$.

Die Risse verliefen sehr steil und entstanden vorwiegend in den Steinen. Der Teil der Wand, der nicht satt auflag, blieb bis zum Versuchsende rissefrei. Kurz vor Erreichen der Maximallast entstanden die ersten Risse im Druckflansch. Die Steine in der Druckecke waren schon stark zerrissen und durch Abklopfen konnte festgestellt werden, dass sich die äusseren Schalen vieler Steine bereits losgelöst hatten. Beim Erreichen der Maximallast spaltete sich der Druckflansch in seiner Ebene; die Horizontalverschiebung betrug $6.3 \mathrm{~mm}$. Das Bild 78 zeigt die Wand in diesem Zustand. In der folgenden Laststufe wurde die Horizontalverschiebung um $1.6 \mathrm{~mm}$ gesteigert. Dabei fiel die Querkraft auf $87 \%$ der Bruchlast zusamen und Teile des Druckflansches fielen ab.

Der Einsturz der Wand ereignete sich während einer Belastungsperiode mit konstant gehaltener Verformung in Abwesenheit des Versuchspersonals.

\subsubsection{Versuch W3}

Der Verlauf von Querkraft und Horizontalverschiebung im Versuch W3 ist im Bild 15 festgehalten. Die zugehörigen Messwerte sind in der Tabelle A3 zusammengestel1t. Die Normalkraft betrug $-415 \mathrm{kN}$ und war gleichmässig verteilt. Die Wand war in jeder Lagerfuge bewehrt.

Die elastische Phase reichte wieder bis zu ca. 50\% der Bruchlast. Die Steifigkeit.war etwas grösser als im Versuch W1, da die untere Platte nun satt auflag. Bei $68 \%$ der Bruchlast und 0.9 mm Horizontalverschiebung lag der maximale Riss im Zugflansch und hatte eine Weite von $0.15 \mathrm{~mm}$. Die Risse traten fast ausschliesslich in den Fugen auf und waren gleichmässig verteilt. Im Zugflansch konzentrierten sich die Verformungen auf einen Riss in der zweiten Mörtelfuge oberhaib der Betonplatte. Dadurch nahm die Rissweite stark zu. Bei 94\% der Bruchlast und einer Verschiebung von $6 \mathrm{~mm}$ erschien der erste Riss im Druckflansch. Die Rissverteilung der Wand bei $19 \mathrm{~mm}$ Horizontalverschiebung ist im Bild 78 gezeigt. Die Horizontalverschiebung konnte nun bis $31 \mathrm{~mm}$ gesteigert werden, bis die Maximallast erreicht war. Gleichzeitig entstanden die ersten Steinrisse in der Druckecke der Wand. Die Maximallast konnte bis zu einer Verschiebung von $50 \mathrm{~mm}$ praktisch ohne Abfall gehalten werden, obwohl sich der Flansch bei $40 \mathrm{~mm}$ Verschiebung spaltete. In dieser Phase waren auch Biegerisse an der Oberkante der oberen Betonplatte zu beobachten. Der maximale Riss im Zugflansch hatte sich auf über $29 \mathrm{~mm}$ geöffnet. Ab ca. $58 \mathrm{~mm}$ Horizontalverschiebung fiel die Querkraft relativ rasch ab. Parallel dazu fielen die Steine in den untersten zwei Schichten in der Druckecke und im Druckflansch auseinander. Das Bruchbild von W3 ist im Bild 27 dargestellt. 
Die grosse Verformungsfähigkeit der Wand W3 ist auf die günstige Wirkung der gut verankerten Bügel zurückzuführen. Dadurch wurde ein frühzeitiges Auseinanderfallen der Druckecke verhindert. Die Bruchlast wurde durch die Bügel allerdings nur wenig erhöht, sie lag $5 \%$ höher als bei W1. Bei einer gleichen Verschiebung von $8 \mathrm{~mm}$ betrug die zugehörige Querkraft in beiden Versuchen $260 \mathrm{kN}$.

\subsubsection{Versuch W4}

Der Verlauf von Querkraft und Horizontalverschiebung im Versuch w4 ist im Bild 16 festgehalten. Die zugehörigen Messwerte sind in der Tabelle A4 zusammengestellt. Die Normalkraft betrug $-423 \mathrm{kN}$ und griff über einen Verteilträger aus Stahl (Bild 8) mit einer Exzentrizität von $0.84 \mathrm{~m}$ - ca. im Viertelspunkt der Wand an. Die Wand war unbewehrt. Für diesen Versuch waren die Flanschen auf $0.9 \mathrm{~m}$ verbreitert worden.

Bereits bei $26 \%$ der Bruchlast war der erste Riss im Zugflansch mit einer Weite von $0.05 \mathrm{~mm}$ zu beobachten. Doch auch in diesem Versuch reichte die elastische Phase bis $2450 \%$ der Bruchlast und $0.6 \mathrm{~mm}$ Horizontalverschiebung. Auf dieser Lasthöhe lag der grösste Riss im Zugflansch in der zweiten Fuge oberhalb der Betonplatte. Seine Grösse betrug $0.15 \mathrm{~mm}$. Im Bereich der Einleitung der Normalkraft waren einige Steinrisse von maximal $0.08 \mathrm{~mm} z u$ beobachten. Bei $74 \%$ der Bruchlast rissen die ersten Steine in der Druckecke der Wand. Im restlichen Bereich waren vorwiegend Fugenrisse festzustellen. Ab $88 \%$ der Bruchlast oder $2.5 \mathrm{~mm}$ Horizontalverschiebung öffnete sich nur noch die fünfte Mörtelfuge oberhalb der Betonplatte im Zugflansch. Bei ca. $9 \mathrm{~mm}$ Verschiebung war die Maximallast erreicht. Gleichzeitig riss der Druckflansch über eine Höhe von sechs Steinen auf. Bild 78 zeigt die Wand $W 4$ in diesem Zustand. Deutlich erkennbar sind der kiaffende horizontale Riss, der vom Zugflansch ausgeht sowie ein bis zwei grosse treppenförmige Risse ungefähr in der Diagonalen. Bei weiterer Steigerung der Horizontalverschiebung blieb die Querkraft praktisch konstant, bei $18 \mathrm{~mm}$ Verschiebung betrug sie noch immer 95\% der Bruchlast. Die beiden oben beschriebenen grossen Risse öffneten sich weiter, so dass das dreieckige Stück Mauerwerk dazwischen nur noch an der oberen Betonplatte klebte. Bei ca. $20 \mathrm{~mm}$ Verschiebung löste es sich von der Betonplatte und fiel auf das darunter1 iegende Mauerstück hinunter. Nun'spaltete sich auch der Druckflansch, und der Flansch schob entlang dieser Trennlinie ab. Dadurch fiel die Querkraft stark ab. Das Bruchbild von w4 ist im Bild 27 festgehalten.

\subsubsection{Versuch W5}

Der Verlauf von Querkraft und Horizontalverschiebung im Versuch W5 ist im Bild 17 festgehalten. Die zugehörigen Messwerte sind in der Tabelle A5 zusammengestellt. Der Verlauf der Vorspannkraft ist aus dem Bild 26 ersichtlich. Die Nomalkraft betrug $-424 \mathrm{kN}$ und griff mit einer Exzentrizität von $1.72 \mathrm{~m}-\mathrm{ca}$. in der Mitte des Druckflansches - an. Die Wand war in jeder Lagerfuge bewehrt und auf der Zugseite vertikal vorgespannt. Auch in diesem Versuch betrug die Breite der Flanschen $0.9 \mathrm{~m}$.

Die Vorspannung auf die Wand wirkte sich sehr günstig auf den Rissbeginn aus, da das Abheben auf der Zugseite behindert war. Bei $45 \%$ der Bruchlast und $1.6 \mathrm{~mm}$ Verschiebung betrug die maximale Rissweite erst $0.05 \mathrm{~mm}$. Es waren durchwegs Fugenrisse im Mittelbereich der Wand. Wie günstig sich die Wand W5 bezüglich der Rissweiten verhalten hat, ist deutlich aus der Abbildung 86 ersichtlich. Leider konnte diese Entwicklung nicht weiter verfolgt werden, da durch einen Defekt in der Servohydraulik ein Zug-Druck-Stoss auf die Wand ausgeübt wurde. Der Stoss dauerte wenige Sekunden und verformte die Wand auf ca. $30 \mathrm{~mm}$ ( $\mathrm{vgl}$. Bild 17). Infolge dieses Stosses waren praktisch alle Fugen und viele Steine gerissen, so dass im weiteren Verlauf des Versuches auf Rissmessungen verzichtet wurde.

Danach wurde eine neue Nullablesung vorgenommen, die Wand wieder vorgespannt und der Versuch von neuem begonnen. Durch den Stoss wurde die Steifigkeit der Wand auf ca. 40\% reduziert (vgl. Bild 17). Durch die Horizontalverschiebung wuchs auch die Vorspannkraft an (vgl. Bild 26). Bei $86 \%$ der Bruchlast war die zweite Mörtelfuge oberhalb der Betonplatte im Zugflansch durchgerissen. Bei $18 \mathrm{~mm}$ Verschiebung war die maximal gemessene Endlast erreicht. Gleichzeitig waren Risse im Druckflansch zu beobachten. Die Querkraft stieg bis zu einer Verschiebung von $21.4 \mathrm{~mm}$ weiter an. Die zugehörige Endlast $V_{E}$ lässt sich auf ca. 365 kN abschätzen. Danach fiel die Querkraft ab. Bei $24 \mathrm{~mm}$ Verschiebung spaltete sich der Druckflansch 
bis hoch hinauf, und die Querkraft fiel auf 93\% der Maximallast ab. Das Rissbild der Wand in dieser Laststufe ist im Bild 79 festgehalten. Die Wand wurde bis ca. $28.5 \mathrm{~mm}$ weiter verformt. Auch in diesem Versuch wurden die Steine in der Druckecke zerstört, und der Flansch schob entlang des Spaltrisses ab. Die Querkraft fiel dabei stark zurück, und der Versuch wurde abgebrochen. Das Bruchbild von W5 ist im Bild 28 dargestellt. Die im Bild erkennbare Spindel diente als Sicherung der oberen Betonplatte.

Die Vorspannkraft fiel jeweils zu Beginn des Versuches um 6 bis $9 \mathrm{kN}$ ab. Dies ist auf Kriechen in der Wand und einen gewissen Schlupf der Verankerung in der unteren Betonplatte (Risse) zurückzuführen. Ab Horizonalverschiebungen von ca. $1 \mathrm{~mm}$ stieg dann die Vorspannkraft kontinuierlich an und erreichte bei ca. $20 \mathrm{~mm}$ ein Maximum von $310 \mathrm{kN}$.

Obwohl die Wand durch einen Stoss der Servohydraulik stark beschädigt worden war, konnte praktisch dieselbe Maximallast erreicht werden wie beim ungeplanten Stoss (vgl. Bild 17). Ein Vergleich der Aufzeichnungen des Plotters während des Stosses und bei der Wiederbelastung ergab im zweiten Versuch eine um $8 \%$ geringere Maximallast gegenüber dem Stoss der Servohydraulik. Diese Differenz kann aber ohne weiteres mit der sehr hohen Belastungsgeschwindigkeit des Stosses erklärt werden.

\subsubsection{Versuch w6}

Die Normalkraft im Versuch w6 betrug $-418 \mathrm{kN}$ und war gleichmässig verteilt. Die Wand war unbewehrt. Sie wurde zyklisch verformt, wie dies im Bild 9 dargestellt ist.

Der Verlauf von Querkraft und Horizontalverschiebung im Versuch w6 für positive Verschiebungen ist im Bild 18 dargestellt. In den Bildern 20 und 21 sind die entsprechenden Kurven der einzelnen Laststufen für positive und negative Verschiebungen festgehalten. Schliesslich zeigt das Bild 24 den Lastabfall in jeder Laststufe in Abhängigkeit der Anzahl Zyklen. Die entsprechenden Messwerte sind in den Tabellen A6 und A8 zusammengestellt.

Bis zu 64\% der Maximallast im ersten Zyklus $\left(V_{E, u(n=0)}\right)$ verhielt sich die Wand w6 linear elastisch. Der grösste Riss lag in der Mitte der Wand und hatte eine Weite von $0.05 \mathrm{~mm}$. Es waren ausschliesslich Risse in den Fugen zu beobachten. Die Querkraft veränderte sich infolge der Zyklen nicht.

Bei $85 \%$ der Maximallast $V_{E, u(n=0)}$ und $1.5 \mathrm{~mm}$ Verschiebung wurde der erste Riss im Zugflansch beobachtet. Seine Weite betrug $0.04 \mathrm{~mm}$. In der Wand war die Rissweite auf maximal $0.15 \mathrm{~mm}$ angestiegen. Während der Zyklen fiel die maximale Querkraft geringfügig ab, während die Rissweiten ungefähr konstant blieben. Bei $3 \mathrm{~mm}$ Verschiebung waren $97 \%$ der Maximallast im ersten Zyklus erreicht. Bei der Erstbelastung wurde der erste Riss im Druckflansch beobachtet. Auch in dieser Laststufe war der Lastabfall infolge der Zyklen gering. Allerdings rissen während der Zyklen mehrere Steine in der Druckecke. In dieser Laststufe wurde die maximale Endlast nach zehn Zyklen gemessen. Sie betrug 94\% der maximalen Querkraft im ersten Zykius. Verglichen mit der Bruchlast des entsprechenden statischen Versuches W1 ergab die zyklische Belastung eine Reduktion der Maximallast $V_{E, u(n=10)}$ von $11 \%$. Die maximale Rissweite betrug $0.6 \mathrm{~mm}$. Bei $6 \mathrm{~mm}$ Verschiebung war die Maximallast $V_{E, u(n=0)}$ erreicht. Gleichzeitig erschienen neue Risse im Druckflansch. Während der Zyklen spaltete sich der Druckflansch für negative Verschiebungen. Die Entwicklung der Rissweiten war unterschiedlich, doch schienen die Steinrisse mehrheitlich anzuwachsen, während die Fugenrisse gleich blieben oder sogar kleiner wurden. Der Zustand der Wand am Ende dieser Laststufe ist im Bild 79 dargestellt. Bei $9 \mathrm{~mm}$ Verschiebung fiel die Querkraft im ersten Zyklus auf 93\% der Maximallast zurück. Während die Maximallast in den ersten sechs Zyklen etwa linear abfiel, nahm der Lastabfall im 7. und im 8. Zyklus bedeutend $z u$, so dass der Versuch im 8. Zyklus abgebrochen wurde. In den sieben Zyklen wurde vor allem der Bereich in der Mitte der Wand stark zerstört. Einzelne Steinschalen fielen aus der Wand, und es waren für beide Querkraftrichtungen Trennlinien in der Wand erkennbar. Das Bruchbild von W6 ist im Bild 28 dargestellt.

Im Bild 21, LS 9, ist sehr schön erkennbar, wie die Kurve "Querkraft-Horizontalverschiebung" während der 
Zyklen - oder im Bruchvorgang - ihre Form änderte. Im ersten Zyklus hatte die Kurve die Form des Buchstabens $S$, wie in den früheren Laststufen. Die Kurve ist bei der Entlastung so lange konkav bezüglich des Ursprungs, bis sich die Risse im Zugflansch geschlossen haben. Infolge der Zerstörung während der Zyklen sank die Wand in sich zusammen (Bild 41), der Flansch öffnete sich nicht mehr, und es entstand eine konvexe Kurve.

Aus den Kurven Querkraft-Horizontalverschiebung in den Bildern 20 und 21 bzw. im Anhang A8 können die folgenden Energien berechnet werden:

- Die während des Verformungsprozesses vom Kolben geleistete Energie:

Sie entspricht dem Integral unter der Kurve Querkraft-Horizontalverschiebung bei der Belastung von $V=0$ bis $V_{A(n=1 / 10)}$ (Summe für positive und negative Querkräfte).

- Die von der Wand dissipierte (absorbierte) Energie:

Sie entspricht der von der Kurve Querkraft-Horizontalverschiebung während eines vollen Zyklus eingeschlossenen Fläche.

Die Wand w6 zeigte bis zur Laststufe 6 ein ungefähr elastisches Verhalten. Während dieser Zeit stieg das Verhältnis dissipierte/geleistete Energie im 10. Zyklus von $10 \%$ auf $20 \%$ an. Sobald sich die Kurve Querkraft-Horizontalverschiebung stärker krümmte (ab LS 7), stieg das Verhältnis deutlich an. Es erreichte in der LS 8 40\% und während des Bruchvorgangs in LS 9 sogar $70 \%$.

\subsubsection{Versuch $W 7$}

Die Normalkraft im Versuch 67 betrug $-1 ' 290 \mathrm{kN}$. und war gleichmässig verteilt. Die Wand war unbewehrt. Sie wurde zyklisch verformt, wie dies im Bild 9 dargestellt ist.

Der Verlauf von Querkraft und Horizontalverschiebung im Versuch W7 für positive Verschiebungen ist im Bild 19 dargestellt. In den Bildern 22 und 23 sind die entsprechenden Kurven der einzelnen Laststufen für positive und negative Verschiebungen festgehalten. Schliesslich zeigt das Bild 25 den Lastabfall in jeder Laststufe in Abhängigkeit der Anzahl Zyklen. Die entsprechenden Messwerte sind in den Tabellen A7 und A9 zusammenges te $11 \mathrm{t}$.

Die Wand verhielt sich bis zu $51 \%$ der Maximallast im ersten Zyklus linear elastisch. Der grösste Riss bei einer Verschiebung von $0.8 \mathrm{~mm}$ befand sich in der Scheibe, seine Weite betrug $0.1 \mathrm{~mm}$. Es waren fast ausschliesslich sehr steile Risse in den Steinen zu beobachten. Die Querkraft fiel infolge der Zyklen nicht ab. Die Last-Verschiebungs-Kurve krümte sich danach nur gering. Die Steifigkeit der Wand blieb sehr hoch. Auch bei $69 \%$ der Maximallast $V_{E, u(n=0)}$ blieb die Querkraft praktisch konstant. Die maximale Rissweite betrug erst $0.2 \mathrm{~mm}$.

Bei einer Anfangslast von 88\% der Maximallast nahm die Querkraft infolge Zyklen um 6\% ab. Der Druckflansch für negative Verschiebungen spaltete sich während der Zyklen. Bei einer Anfangslast von 99\% der Maximallast und $3 \mathrm{~mm}$ Verschiebung erschien auch der erste Riss im Druckflansch für positive Verschiebungen. Entsprechend den Erfahrungen in früheren Versuchen war damit die maximale Endlast $v_{E, u}(n=10)$ erreicht. Während der Zyklen riss auch der zweite Flansch, und die Querkraft fiel um 11\% ab. Die maximale Rissweite betrug $0.5 \mathrm{~mm}$. Der Zustand der Wand in dieser Phase ist im Bild 79 festgehalten. Die Anfangslast $V_{E(n=0)}$ konnte bis zur Verschiebung von $4 \mathrm{~mm}$ noch gering gesteigert werden. Es erschienen zahlreiche neue Risse. Während der Zyklen wurde wie beim Versuch w6 vor allem der Bereich in der Mitte der Wand stark zerstört. Die Last sank in den Zyklen um 25\% ab. Da die Last-Verschiebungs-Kurve so steil abfiel, wurde der Versuch an dieser Stelle abgebrochen. Das Bruchbild von $W 7$ ist im Bild 28 dargestellt.

In der Laststufe 4 nahm die Querkraft während der Zyklen geringfügig zu. Dies ist auf eine Verschiebung der Hysteresis entlang der Achse $\delta_{h}$ zurückzuführen. 
Ab Laststufe 7 ergaben sich Differenzen in der Zentralsymmetrie der Last-Verformungs-Kurven. Bei negativen Verschiebungen war der Betrag der Querkraft kleiner als bei positiven Verschiebungen. Die Ursachen dafür sind nicht genau bekannt. Wahrscheinlich liegt der Grund in der unterschiedlichen Lasteinleitung der Querkraft auf Zug bzw. auf Druck.

Im Versuch W7 zeigten die Last-Verschiebungs-Kurven keine S-Form, da der Zugflansch nie abhob.

Aus den Bildern 22 und 23 bzw. Anhang $A 9$ lässt sich die geleistete bzw. die dissipierte Energie für die Wand W7 berechnen (vgl. Abschnitt 4.2.7). Wie die Bilder 22 und 23 zeigen, verhielt sich die Wand W7 praktisch in allen Laststufen elastisch. Das Verhältnis dissipierte Energie/geleistete Energie blieb dementsprechend gering. In der Laststufe 4 betrug es im 10. Zyklus nur gerade 3\% und wuchs bis Laststufe 8 stetig auf $17 \%$ an. In der Laststufe 9 war das Verhältnis maximal und betrug 27\%. Damit war die EnergieDissipation (Energie-Absorption) bei gleicher Horizontalverschiebung im Versuch w6 mit kleiner Normalkraft sowohl relativ als auch absolut, z.T. bedeutend höher als im Versuch w7 mit hoher Normalkraft.

\subsection{Randverschiebungen}

Die Randverschiebungen stellen integrale Grössen der weiter hinten beschriebenen Verzerrungen dar. Sie sind die Verschiebungen des ganzen Tragwerks, währeṇd die Verzerrungen Aufschluss über lokale Vorgänge geben.

\subsubsection{Horizontale Randverschiebungen}

Die horizontalen Randverschiebungen sind in den Bildern 29 bis 35 dargestellt. Auf der vertikalen Achse sind die Messstellen (MS) in massstäblichem Abstand aufgetragen. Die Messstellennummern sind im Bild 29 eingetragen (vgl. Bild 10). Die Messwerte der Messstellen 32 und 40 konnten nicht ausgewertet weriden.

Bei den unbewehrten Wänden weisen die Kurven z.T. beachtliche Krümmungen auf. Im Druckflansch rühren diese Krümmungen im unteren Flanschbereich daher, dass das Druckfeld den freien Mauerwerksrand hinausdrückt. Im Zugflansch können die gleichen Phänomene, jedoch weniger ausgeprägt, im oberen Flanschbereich einen Einfluss haben; oder aber der Knick in der Kurve entsteht bei einer Diskontinuität im Flansch, beispielsweise bei einem grossen Riss wie im Versuch W4 (Bild 32). Beim Knick im "Zugflansch" im Versuch W2 (Bild 30) waren in der Laststufe 11 Biegerisse mit einer maximalen Weite von $0.25 \mathrm{~mm}$ zu beobachten.

Bei den bewehrten Wänden verliefen die Kurven geradlinig oder nur leicht gekrümmt. Die Lagerfugenbewehrungen mit den Verankerungsplatten stützten die Ränder gut ab.

Die Versuchskörper mit zyklischer Belastung waren unbewehrt und zeigten deshalb ein ähnliches Verhalten wie in den Versuchen mit progressiver Belastung. Die Krümungen traten in diesen Versuchen jedoch an den oberen und unteren Flanschenden auf, weil die unelastischen Verformungen bei der Aenderung der Lastrichtung nicht vollständig rückgängig gemacht wurden. So wies der Zugflansch in beiden Versuchen bei maximaler positiver Verschiebung $\delta_{h}$ noch negative Randverschiebungen auf. Infolge der Zyklen verlängerten sich die Wände in horizontaler Richtung um bis $z u 10 \mathrm{~mm}$, die Wandstruktur wurde also aufgelockert.

\subsubsection{Vertikale Randverschiebungen}

Die vertikalen Randverschiebungen sind in den Bildern 36 bis 42 dargestellt. Auf der horizontalen Achse sind die Messstellen (MS) in massstäblichem Abstand aufgetragen. Die Messstellennummern sind im Bild 36 eingetragen ( $\mathrm{vgl}$. Bild 10).

A1len Versuchen mit kleiner Normalkraft, W1, W3, W4, W5 und W6, war gemeinsam, dass sich die Wände mit zunehmender Horizontalverschiebung $\delta_{h}$ vertikal verlängerten. Die grössten vertikalen Verschiebungen wurden 
in den duktilen Versuchen W3 und W4 erreicht. Beim Versuch W3 ist das auf die Lagerfugenbewehrung, beim Versuch w4 auf die Biegung zurückzuführen.

Bei den Versuchen mit grosser Normalkraft, W2 und W7, wurden nur Stauchungen beobachtet.

Infolge der zyklischen Verformungen nahmen die vertikalen Randverschiebungen ab. Im Versuch W7, Laststufe 9, ist auch gut ersichtlich, wie vor allem der mittlere Bereich der Wand zerstört und deshalb gestaucht wurde.

Beim Versuch W2 fällt die unregelmässige Form der Kurven auf. Dies ist auf die Unebenheiten zwischen unterer Betonplatte und Aufspannboden zurückzuführen (vgl. 4.2.3). Das gleiche Verhalten, aber viel weniger ausgeprägt, ist auch im Versuch w1 zu beobachten.

Aus diesen Bildern ist klar ersichtlich, in welchen Fällen eine vertikale Bewehrung gedehnt würde und damit Zug erhielte. Nur dort würde sie eine Erhöhung der Bruchlast bringen.

\subsection{Relativverschiebungen zwischen Wand und Betonplatten}

Die Relativverschiebungen zwischen Wand und Betonplatten sind in den Bildern 43 bis 49 dargestellt. Diese Messungen geben Auskunft dárüber, ob die der oberen Betonplatte aufgezwungenen Horizontaiverschiebungen auch tatsächlich auf die Wand übertragen wurden. Wie die Bilder zeigen, kam es in keinem Versuch zu einem Abgleiten zwischen Wand und Betonplatten.

Im Versuch w1 scheint lokal beim Messpunkt 2 ein Gleiten stattgefunden zu haben. Tatsächlich wurden im Versuch in diesem Bereich auch Risse in der Mörtelfuge zwischen wand und Platte beobachtet. Auch im Versuch $W 4$ waren an dieser Stelle Risse zu sehen.

Infolge der zyklischen Verformungen im Versuch w6 traten beim Messpunkt 4 auch negative Verschiebungen auf. Sie hatten ungefähr den gleichen Betrag wie diejenigen des Messpunktes 2.

\subsection{Verzerrungen}

$D i$ Verzerrungen sind in den Bildern 50 bis 70 in jeweils drei Horizontalschnitten durch die Wand dargestellt. Durch diese drei Schnitte erhält man die Verteilung der Verzerrungen bei der Einleitung der Querkraft in die Wand, ungefähr in der Mitte der Wand und bei der Abgabe der Querkraft an die untere Betonplatte. Auf der Abszisse sind die Elementschwerpunkte des Messnetzes in massstäblichem Abstand eingetragen (vgl. Bild 10). Die vertikalen Achsen entsprechen den Aussenkanten der Flanschen. Form und Abmessung der Wände sind in den Bildern angedeutet.

Bei der Interpretation der Messresultate ist zu beachten, dass die Verzerrungen Mittelwerte über den Messbereich "Stein + Fuge" darstellen. Die Verschiebungen verteilen sich aber je nàch Richtung unterschiedlich auf Stein und Fuge ( $\mathrm{vgl},[1])$ :

$-\varepsilon_{x}:$

Dehnung nur in der Lagerfuge

Stauchung im Stein und in der Lagerfuge

$-\varepsilon_{y}:$

Dehnung je nach Lage des Risses in der Stossfuge oder im Stein

Stauchung nur in der Stossfuge 


\section{$\underline{4.5 .1 \text { Dehnungen }} \varepsilon_{\mathrm{x}}$}

Die Dehnungen $\varepsilon_{x}$ beziehen sich auf die Richtung senkrecht zu den Lagerfugen. Bei kleiner Normalkraft wurden vor. allem Dehnungen gemessen. Nur der Wandteil in der Nähe des Druckflansches wurde gestaucht. Bei grosser Normalkraft dagegen wurden alle Bereiche der Wand gestaucht. Dies stimmt gut mit den Resultaten der vertikalen Randverschiebungen überein.

Die Lagerfugenbewehrung im Versuch W3 hatte keinen Einfluss auf die Verteilung der Dehnungen im Vergleich zum Versuch W1. Dagegen war der Verlauf der Kurven im Versuch W3 etwas regelmässiger.

Durch die zyklischen Verformungen nahmen die Dehnungen ab bzw. die Stauchungen zu.

\subsubsection{Dehnungen $\varepsilon_{y}$}

Die. Dehnungen $\varepsilon_{y}$ beziehen sich auf eine Richtung parallel zu den Lagerfugen. Im allgemeinen blieben die Dehnungen $\varepsilon_{y}$, mit Ausnahme einzelner Spitzen, klein. Die grössten Dehnungen wurden in der halben Höhe der Wand (Schnitt 2-2) gemessen, weil dort die Behinderung der Querdehnung durch die Betonplatten am geringsten war.

Bei den unbewehrten Versuchskörpern war der Verlauf der Dehnungen $\varepsilon_{y}$ recht unregelmässig. Es wurden grosse Spitzenwerte bis zu $\varepsilon_{y, \max }=0.009$ im Versuch $W 1$ gemessen. Dies bedeutet, dass sich die Verschiebungen in einem Riss konzentrierten. Die grössten Dehnungen $\varepsilon_{y}$ wurden häufig in der Nähe der Wandränder gemessen.

Bei den bewehrten Versuchskörpern war der Verlauf der Dehnungen sehr ausgeglichen. Als maximale Dehnungen wurden im Versuch $W 3 \varepsilon_{y, \max }=0.001$ und im Versuch $W 5 \varepsilon_{y \text {, max }}=0.002$ gemessen. Damit lag die Dehnung in den Bügeln (Lagerfugenbewehrung) noch deutlich unterhalb der Streckgrenze des Stahles.

Während den zyklischen Verformungen nahmen die Dehnungen $\varepsilon_{y}$ zu (vgl. Bild 66: W6 Laststufe 8). Die Struktur der Wände wurde aufgelockert.

\subsubsection{Schiebungen $\gamma_{x y}$}

Die Schiebungen $\gamma_{x y}$ beziehen sich auf ein Koordinatensystem mit Achsen senkrecht und parallel zu den Lagerfugen. Die Schiebungen stellen die betragsmässig grössten Verzerrungen dar.

Wie schon bei den Dehnungen $\varepsilon_{y}$ bewirkte eine Lagerfugenbewehrung auch hier vor allem einen gleichmässigeren Verlauf der Schiebungen. Bei der Lastabgabe an die untere Betonplatte (Schnitt 3-3) waren die Schiebungen zudem weniger stark konzentriert.

In den zyklischen Versuchen nahmen die Schiebungen gesamthaft nicht zu, da das Integral der Schiebungen ungefähr der aufgezwungenen Horizontalverschiebung $\delta_{h}$ entsprach. Dagegen konnte eine Umverteilung der Schiebungen in Richtung Mitte der Wand beobachtet werden. Während die Schiebungen in den Schnitten 1-1 und 3-3 eher sanken, und z.T. das Vorzeichen wechselten, nahmen sie im Schnitt 2-2 zu (vgl. Bild 67: W6, Laststufe 8 und Bild 70: W7, Laststufe 9). Dadurch ist auch die Zerstörung vor allem des mittleren Bereichs der Wände W6 und W7 erk1ärt.

Im Versuch W5, Bild 64 Schnitt 3-3, ist zu beachten, dass dort ein Teil der Achse herausgeschnitten wurde, um die Schiebungen in der Laststufe 14 noch darstellen zu können. Der Verlust an Steifigkeit durch den Stoss hatte in diesem Bereich der Wand sehr grosse Schiebungen zur Folge.

Im Versuch W2 ist wieder der Einfluss der ungenügenden Lagerung zu erkennen. Vor allem in den Schnitten 2-2 und 3-3 entstand ein "Loch" in den Kurven. 
Die Hauptdehnungen sind in den Bildern 71 bis 77 dargestellt. Pro Versuch sind jeweils zwei Laststufen festgehalten. Das erste Bild zeigt die Hauptdehnungen in der elastischen Phase, das zweite diejenigen bei der Maximallast. Es ist zu beachten, dass sich die Massstäbe der beiden Bilder in einer Zehnerpotenz unterscheiden.

In der elastischen Phase ist die Grösse der Hauptdehnungen bei gleichmässig verteilter Normalkraft in allen Elementen ähnlich. Die Hauptrichtungen sind ungefähr parallel zueinander. Betragsmässig überwiegen in dieser Phase die Stauchungen. Eine Ausnahme bilden die Versuche $W 1$ und W2, bei denen die Lagerung der unteren Platte ungenügend war. Vor allem im Versuch w2 weichen die Hauptdehnungen sehr stark vom Sollwert (vgl. W7, Bild 77) ab. Es lässt sich eine Art Bogenwirkung über dem ungenügend gestiutzten Teil der unteren Platte erkennen.

Im Versuch W4 war die Normalkraft konzentriert mit einer Exzentrizität von $0.84 \mathrm{~m}$. Im Versuch w5 ist vor allem die Einleitung der Vorspannkraft aus der unteren Platte erkennbar.

Mit zunehmender Horizontalverschiebung werden auch die Hauptdehnungen unregelmässig. Die inneren Kräfte in der Wand lagern sich um. In den Laststufen mit Maximallast ist eine Konzentration der Dehnungen in der Druckecke und/oder entlang der Druckdiagonalen zu erkennen. Betragsmässig überwiegen die Dehnungen. In den bewehrten Versuchskörpern ist die Dehnungsverteilung bedeutend gleichmässiger.

Die Richtung der maximalen Stauchungen verläuft in Versuchen mit kleiner Normalkraft flacher als bei Versuchen mit grosser Normalkraft. In allen Versuchen kann aber beobachtet werden, dass die Richtung der Hauptstauchung mit zunehmender Horizontalverschiebung flacher wird. Dies geht parallel zum Abbau der Zugfestigkeit im Mauerwerk vor sich.

In Versuchen mit zyklischer Belastung neigen sich die Hauptdehnungen in den Wandecken z.T. der Querkraftrichtung entgegen. In diesen Bereichen haben die Schiebungen $\gamma_{x y}$ das Vorzeichen gewechselt.

Wie Untersuchungen an Mauerwerkskörpern unter gleichmässigen Spannungen [1] zeigten, weichen die Richtungen der Hauptdehnungen in der ungerissenen Phase der Versuche nur relativ wenig (maximal \pm 5 bis 10 Altgrad) von den Richtungen der Hauptspannungen ab. Sobald ein Riss im betrachteten Bereich entsteht, verändert das den Dehnungszustand und damit auch die Richtung der Hauptdehnungen z.T. vollständig. Da über Risse hinweg grosse Dehnungen auftreten, und gleichzeitig die Stauchungen gering bleiben, dominieren die Risse den Verzerrungszustand. Deshalb ergibt der Verlauf der Hauptdehnungen in der gerissenen Phase der Versuche ein sehr unregelmässiges Bild. Er darf keineswegs mit dem Verlauf der Hauptspannungen gleichgesetzt werden.

\subsection{Rissverhalten}

Die Rissbilder der Versuche W1 bis $W 7$ sind in den Abbildungen 78 und 79 festgehalten. In den Bildern 80 bis 85 ist die Entwicklung einzelner Risse während des Versuchs (ohne W5) in Abhängigkeit der Horizontalverschiebung dargestellt. Bild 86 zeigt einen Vergleich der maximal gemessenen Rissweiten aller Versuche. Es konnten nur Rissweiten in den Lagerfugen und in den Steinen gemessen werden, da die Stossfugen gegen die Wandoberfläche hin nicht vermörtelt waren. Rissweiten in den Stossfugen können über die Dehnungen $\varepsilon_{y}$ abgeschätzt werden, indem die Steinverformungen vernachlässigt werden.

Es sind Risse in verschiedenen Bereichen der Versuchskörper dargestellt. Für die Scheibe sind, wenn vorhanden, Fugen- und Steinrisse mit möglichst grosser Rissweite abgebildet. Auf dem Zugflansch ist mindestens 
der Riss mit maximaler Weite dargestellt, und im Druckflansch mindestens der zuerst aufgetretene Riss (vgl. Abschnitt 4.2 Trag- und Bruchverhalten). Die Lage der Risse ist im Bild eingezeichnet. Zusätzlich ist auch $R_{\max }$ als Unhuillende der maximal beobachteten Rissweiten eingetragen. Dank dieser Darstellung ist es möglich zu verfolgen, welche Risse zu welchem Zeitpunkt des Versuchs die maximale Rissweite aufweisen.

Bei kleiner Normalkraft bildeten sich vorwiegend Fugenrisse, bei grosser Normalkraft Steinrisse. Vertikales Oeffnen des Zugflansches konzentrierte sich immer in einer Fuge.

Bei kleinen Verschiebungen $\delta_{h}$ bildeten die Fugenrisse in der Scheibe des Versuchskörpers W1 das Maximum. Ab ca. $5 \mathrm{~mm}$ Horizontalverschiebung lag die maximale Rissweite dann im Zugflansch.

Im Versuch W2 wurden fast ausschliesslich Steinrisse beobachtet. Bei kleinen Verschiebungen machten sich die Zwängungen infolge ungleichmässiger Lagerung der Platte stark bemerkbar, die Rissweiten waren bedeutend grösser als bei W7. Der Riss Numer 9 war ein Biegeriss im Zugflansch. Er lag an der Stelle der maximalen Krümmung des Flansches (vgl. Abschnitt 4.4.1 Horizontale Randverschiebungen).

Im Versuch W3 lag die maximale Rissweite von Versuchsbeginn an im Zugflansch. Dies wurde durch zwei Umstände begünstigt. Die Fugenbewehrung verteilte die Risse in der Scheibe gleichmässig, deshalb war dieses Maximum kleiner als bei $W 1$. Durch die Fugenbewehrung wurde das Abheben aber auch deutlicher auf den Zugflansch konzentriert, und die Rissweiten stiegen dort stark an. Es sind nur Fugenrisse abgebildet, da die Steinrisse erst bei grösseren Horizontalverschiebungen auftraten.

Wegen des Biegemomentes lag im Versuch W4 die maximale Rissweite von Anfang an im Zugflansch. Bei ca. $2 \mathrm{~mm}$ Horizontalverschiebung verlagerte sich der maximale Riss von der zweiten in die fünfte Fuge über der Betonplatte.

Im Versuch W5 wurden die Risse wohl nachgezeichnet, jedoch nicht gemessen. Der Rissbeginn war bedeutend später als in allen anderen Versuchen. Im Zugflansch konzentrierte sich das Abheben wieder in eine Lagerfuge.

Im Versuch w6 lag die maximale Rissweite bei kleinen Verschiebungen in den Fugen der Scheibe, bei grossen Verschiebungen dagegen in den Steinen der Druckecke. Der Zugflansch wurde nie massgebend. Während der Zyklen blieb die Weite der Fugenrisse konstant oder nahm ab, diejenige der Steinrisse nahm zu.

Im Versuch W7 wurden nur Steinrisse in der Scheibe und im Druckflansch beobachtet.

Der Vergleich der maximalen Rissweiten im Bild 86 zeigt, dass die Kurven bei kleinen Horizontalverschiebungen nahe beisammen liegen (Ausnahmen: W2 - Zwängungen und W4 - Biegung). Ab ca. $3 \mathrm{~mm}$ Verschiebung fächern sich die Kurven auf. Die grössten Rissweiten treten ab da bei Biegung und kleiner Normalkraft auf, die kleinsten bei grosser Normalkraft. Beim Versuch w5 mit vertikaler Vorspannung lag die maximale Rissweite bei ca. $1.5 \mathrm{~mm}$ Horizontalverschiebung deutlich tiefer als bei allen anderen Versuchen. 


\section{Zusammenfassung}

Im Rahmen des Forschungsprojektes "Trag- und Bruchverhalten von Mauerwerksscheiben" wurden am Institut für Baustatik und Konstruktion der Eidgenössischen Technischen Hochschule Zürich Versuche an sieben Mauerwerkswänden durchgeführt. Diese Versuche hatten zum Ziel, das Verhalten von Mauerwerksscheiben unter Querkraft, Normalkraft und Bjegemoment zu untersuchen. Die Parameter waren die Normalkraft, das Biegemoment, die Bewehrung und die Belastungsart (progressive oder zyklische Belastung). Das Versuchsprogramm ist in der Tabelle 1 zusammengestellt. Es wurden eine einzige Steinsorte, ein Modulbackstein, und nur ein Zementmörtel verwendet. Die Wände wurden mit einer Normalkraft in bestimmter Exzentrizität belastet. Die Normalkraft blieb während des ganzen Versuchs konstant. Anschliessend wurde die Horizontalverschiebung des oberen Wandendes stufenweise (progressiv oder zyklisch) bis zum Bruch der Wand gesteigert. Es wurden die Lasten, die Horizontalverschiebung, die Randverschiebungen und die Verschiebungen in der Scheibenebene gemessen und das Rissverhal ten beobachtet.

Die Versuchsresultate lassen sich wie folgt zusammenfassen:

- Mauerwerksscheiben zeichnen sich durch eine grosse Steifigkeit im elastischen Bereich aus. Die Steifigkeit war näherungsweise unabhängig von den Versuchsparametern. Die elastische Phase reichte bis ca. zur halben Bruchlast.

- Die Bruchquerkraft ist vor allem eine Funktion der Normalkraft. Im untersuchten Bereich nahm die Bruchlast mit steigender Normalkraft ebenfalls zu.

- In den Versuchen mit progressiver Belastung wurde der Bruch durch die Zerstörung der Steine in der Druckecke der Wand eingeleitet. In keinem der Versuche wurde ein Abgleiten der ganzen Wand entlang den Fugen beobachtet.

- Die Duktilität der Wände hängt von der Normalkraft und von der Bewehrung ab. Unter kleinen Normalkräften zeigen selbst unbewehrte Mauerwerkswände ein relativ duktiles Verhal ten. Mit steigender Normalkraft nimmt die Bruchverschiebung stark ab, und die Wände zeigen ein sprödes Verhalten. Durch Einlegen einer gut verankerten Lagerfugenbewehrung konnte die maximal erreichbare Horizontalverschiebung stark erhöht werden.

- Greift gleichzeitig zur Normalkraft ein in gleicher Richtung wie die Querkraft wirkendes Biegemoment in der Wandebene an, so wird die Bruchquerkraft reduziert.

- Das Einlegen einer Lagerfugenbewehrung allein führte beim untersuchten statischen System und Normalkraftniveau nur zu einer sehr geringen Erhöhung der Bruchlast.

- Zyklische Belastungen, wie sie durch Wind und Erdbeben entstehen, hatten bis zu einer Höhe der halben statischen Bruchlast keinen Einfluss auf das Verhalten des Tragwerks.

- Die zyklische Belastung reduzierte die Bruchlast des Tragwerks um 10 bis $15 \%$ im Vergleich zum Versuch mit progressiver Belastung. Die Verformungsfähigkeit der Wände wurde stark vermindert.

- Die Mauerwerkswand mit relativ kleiner Normalkraft (W6) wies unter zyklischer Belastung eine 2.T. bedeutend höhere Energie-Dissipation auf als diejenige mit hoher Normalkraft (W7).

- In den Versuchen mit zyklischer Belastung wurde der Bruch vor allem durch die Zerstörung der Steine in der Mitte der Wand eingeleitet.

- Die im Versuch W5 eingesetzte vertikale Vorspannung erhöhte die Bruchlast bedeutend und wirkte sich günstig auf das Rissverhalten der Wand aus. 
- Bei kleiner Normalkraft traten nur Fugenrisse in der Scheibe und im Zugflansch auf. Erst in der Nähe der Bruchlast rissen auch Steine in der Druckecke der Wand. Bei hoher Normalkraft waren fast ausschliesslich sehr steile Risse in den Steinen zu beobachten.

- Der Rissbeginn lag in allen Versuchen ungefähr bei der halben Bruchlast. Für kleine Horizontalverschiebungen hing die maximale Rissweite in erster Linie von der Horizontalverschiebung und vom Biegemoment ab.

Die Versuchsresultate werden in späteren Arbeiten interpretiert und diskutiert. 
Résumé

Dans le cadre d'un projet de recherche sur le comportement des parois en maçonnerie, on a procédé, à l'institut de statique et de construction de l'EPF Zürich, à des investigations expérimentales sur sept parois en briques en terre cuite. Le but de ces essais était d'examiner le comportement des parois en maçonnerie chargées par un effort tranchant, un effort normal et parfois un moment de flexion. Comme paramètres, on a choisi l'effort normal, le moment de flexion, l'armature et le mode de chargement (progressif ou cyclique). Le programme des essais est résumé dans le tableau 1. Les briques utilisées sont du même type, proviennent de la même fournée et on été hourdées au mortier de ciment. D'abord, les parois ont été chargées par un effort normal avec une certaine excentricité. L'effort normal a été maintenu constant pendant toute la durée de l'essai. Après, on a augmentê le déplacement horizontal de l'extrêmité supérieure des parois (application de déformations progressive ou cyclique) jusqu'à la rupture des parois. Durant les essais on a mesuré les charges, le déplacement horizontal, les déformations des bords des parois, les déplacements dans le plan des parois, de plus on a observé les fissures et estimé leur largeur.

Les résultats des essais peuvent être résumés de la façon suivante:

- Dans la phase êlastique de chargement (environs jusqu'à la moitié de la charge de rupture), les parois en maçonnerie montrent une grande rigidité. La rigidité était indépendante des paramętres des essais.

- L'effort tranchant ultime est surtout fonction de l'effort nomal. Dans le domaine examiné, la charge de rupture d'effort tranchant a été augmentée par une élévation de l'effort normal.

- Dans les essais avec un mode de chargement progressif, la rupture a débutē par l'écrasement des briques dans la zone de compression des parois. On n'a jamais observê un glissement de toute la paroi dans les lits de pose.

- La ductilité des parois dépend de l'effort normal et de l'armature. Chargées par un faible effort normal, mẻme les parois non armées montraient un comportement assez ductile. L'augmentation de l'effort norma? diminue rapidement la déformation ultime et les parois montrent un comportement fragile. Avec l'utilisation d'armature dans les lits de pose, la déformation de rupture a été fortement augmentée presque sans perte d'effort tranchant. Cette armature était bien ancrée.

- L'action combinée de l'effort normal et du moment de flexion (même orientation pour le moment de flexion et pour l'effort tranchant) a diminué l'effort tranchant ultime.

- Pour le systême statique et pour le niveau d'effort normal qui ont été testés, l'armature des lits de pose a produit une augmentation négligeable de l'effort tranchant ultime.

- Une sollicitation cyclique appliquée avec une charge au maximum équivalente à la moitié de la charge de rupture statique $n$ 'influençait pas le comportement des parois.

- Le chargement cyclique a diminué l'effort tranchant ultime de 10 à $15 \%$ en comparaison avec l'essai de chargement progressif. La réduction du déplacement horizontal ultime a été considérable.

- Dans le cas de chargement cyclique, les parois en maçonnerie chargées avec un faible effort normal ont montré une dissipation d'énergie considérablement plus grande que les parois chargées avec un grand effort normal.

- Dans les essais de chargement cyclique, la rupture a débuté par l'écrasement des briques dans la zone centrale des parois. 
- La précontrainte verticale de la paroi W5 a augmenté considérablement l'effort tranchant ultime et a eu une influence favorable sur la fissuration.

- Dans le cas de petits efforts normaux, les fissures n'ont êté observées que dans les lits de pose de la paroi et dans l'aile où s'exerce la force traction. Près de la charge de rupture, un phénomène nouveau apparait: Des briques dans la zone de compression se cassent. Dans le cas de grands efforts normaux, des fissures plus raides dans les briques ont êté observêes.

- Dans tous les essais, le début de la fissuration est apparu à peu près avec une charge égale à la moitié de la charge de rupture. Pour de petits déplacements horizontaux, la largeur maximum des fissures dépend principalement du déplacement horizontal et du moment de flexion.

Dans un prochain travail il est prévu d'analyser et d'interpréter les résultats des essais. 
As part of a research project on the behaviour of masonry shear walls, carried out at the Swiss Federal Institute of Technology Zürich, experiments on seven brick shear walls were performed. The objective of these tests was to investigate the behaviour of masonry walls under shear, normal force and bending moment. The test parameters were the normal force, the bending moment, the reinforcement and the type of loading (progressive or cyclic). The test progranme is surmarized in table 1. Only one type of brick and cement mortar were used. Firstly, the walls were loaded by normal force with a given eccentricity. The normal force was kept constant during the whole test. The horizontal deflection of the upper end of the wall was then increased (progressive or cyclic deformation history) up to failure of the wall. The loads, the horizontal deflection, the deformation of the wall edges, the inplane deformations and the cracking behaviour were noted.

The results of these tests may be summarized as follows:

- In the elastic stage of loading (up to about half the ultimate load) masonry shear walls show a large stiffness. It did not depend on the test parameters.

- The ultimate shear load is greatly influenced by the normal force. Within the investigated range, the ultimate shear load was increased with higher normal force.

- In tests with progressive loading, failure was initiated by the crushing of the bricks in the compression corner of the walls. Sliding of the whole wall along the joints was never observed.

- The ductility of the shear walls depends on the normal force and on the reinforcement. Under low normal forces, even unreinforced shear walls show a relatively ductile behaviour. With increasing normal force the ultimate deformation decreases rapidly and the walls show a brittle behaviour. Using a well anchored bed joint reinforcement, the deformation at failure was substantially increased.

- Combined action of a normal force and a bending moment (same loading direction as the shear force) reduced the ultimate shear load.

- For the investigated static system and level of normal force, bed joint reinforcement alone produced only a negligible increase of the ultimate shear load.

- Cyclic loadings up to half the ultimate static shear load had no influence on the behaviour of the walls.

- Cyclic loading reduced the ultimate shear load by 10 to $15 \%$ in comparison to the tests with progressive loading. The horizontal deflection at failure was considerably decreased.

- Under cyclic loading, the lightly loaded wall w6 showed a considerably larger energy-dissipation than the wall w7 loaded by a high normal force.

- In the tests with cyclic loading, failure was inftiated by crushing of the bricks in the central part of the wall.

- Vertical post-tensioning of specimen W5 increased the Ultimate shear load considerably and had a favourable influence on the cracking behaviour.

- Under low normal force, cracks appeared only in the joints of the wall and the tension flange. Close to the ultimate load, bricks in the compression corner of the wall began to crack. Under high normal force, only very steep cracks in the bricks were observed.

- In all the tests the outset of cracking was close to half the ultimate load. For small horizontal deflections, the maximum crack width depended mainly on the horizontal deformation and the bending moment.

The test results will be analysed in a future report. 
Verdankungen

Der vorliegende Bericht wurde im Rahmen des Forschungsprojektes "Trag- und Bruchverhalten von Mauerwerksscheiben" am Institut für Baustatik und Konstruktion der Eidgenössischen Technischen Hochschule Zürich ausgearbeitet. Für die grosszügige finanzielle Unterstützung möchten die Verfasser dem

Verband Schweizerischer Ziegel- und Steinfabrikanten

aufrichtig danken.

Das Forschungsprojekt wird von einer Beratenden Kommission begleitet, die aus den folgenden Mitgliedern besteht:

Herren H. Gubler, W. Nydegger, R. Porubsky (Nachfolger: Dr. E. Amrein), E. Reinle, W. Santi, E. Setz, Dr. J. Sigg.

Allen Mitwirkenden sei an dieser Stelle für ihre Mitarbeit bestens gedankt.

Ein weiterer Dank gilt der Firma

Bekaert (Switzerland) AG

für die kostenlose Lieferung der Lagerfugenbewehrung. Die Bewehrung musste für diese Versuche in Speziallängen hergestellt werden.

Die Herstellung der Versuchskörper sowie die Durchführung der Normprüfungen an Stein und Mörtel oblagen dem Prüf- und Forschungsinstitut des VSZS in Sursee. Die Verfasser möchten dessen ehemaligem Leiter, Herrn R. Porubsky, sowie den übrigen beteiligten Mitarbeitern des P+F für ihr Entgegenkormmen und ihre Mitarbeit bestens danken.

Bei der Versuchsdurchfuhrung haben die Herren K. Bucher, J. Schönjahn und A. Muttoni mitgearbeitet. Herr Schönjahn wirkte auch bei der Versuchsauswertung mit. Herr M. Baumann hat die messtechnischen Probleme bearbeitet. Herr L. Sieger zeichnete die Bilder und Frl. S. Burki schrieb die Druckbogen. Für die gute Mitarbeit sei den Genannten bestens gedankt.

Herrn R. Caflisch möchten wir speziell für die administrative Betreuung des Forschungsprojektes sowie für seine zahlreichen Ratschläge während der Versuchsvorbereitung danken. 
Bezeichnungen

\section{Geometrische Grössen}

$\begin{array}{ll}x & \text { Koordinatenachse senkrecht zur Lagerfuge } \\ y & \text { Koordinatenachse parallel zur Lagerfuge } \\ u & \text { Verschiebung in x-Richtung } \\ v & \text { Verschiebung in } y \text {-Richtung } \\ \text { e } & \text { Exzentrizität der Normalkraft in Wandebene } \\ \text { R } & \text { Rissweite } \\ R_{\text {max }} & \text { maximal gemessene Rissweite } \\ \text { A } & \text { Stabquerschnittsfläche } \\ \text { A eff } & \text { effektive Stabquerschnittsfläche (Gewichtsmessung) } \\ \text { I } & \text { Stablänge } \\ \text { H } & \text { Wandhöhe }\end{array}$

\section{Kraftgrössen}

$\begin{array}{ll}N & \text { Normalkraft } \\ N_{e x} & \text { an Kraftmessdosen gemessene Normalkraft } \\ V & \text { Querkraft } \\ V_{e x} & \text { an Kraftmessdosen gemessene Querkraft } \\ V_{u} & \text { maximal gemessene Querkraft, Bruchlast } \\ V_{A} & \text { Querkraft zu Beginn einer Laststufe } \\ V_{E} & \text { Querkraft am Ende einer Laststufe } \\ V_{A, u} & \text { maximal gemessene Querkraft zu Beginn einer Laststufe } \\ V_{E, u} & \text { maximal gemessene Querkraft am Ende einer Laststufe } \\ P & \text { Vorspannkraft } \\ P_{0} & \text { Anfangsvorspannkraft }\end{array}$

Materialwerte, Spannungen

$\begin{array}{ll}\sigma & \text { Normalspannung } \\ E & \text { Elastizitätsmodul } \\ E_{s} & \text { Elastizitätsmodul des Bewehrungsstahls } \\ f_{y, s} & \text { statischer Wert der Streckgrenze des Bewehrungsstahls } \\ f_{y, d} & \text { dynamischer Wert der Streckgrenze des Bewehrungsstahls } \\ f_{s t, s} & \text { statischer Wert der Zugfestigkeit des Bewehrungsstahls } \\ f_{s t, d} \quad \text { dynamischer Wert der Zugfestigkeit des Bewehrungsstahls } \\ \text { Verformungsgrössen }\end{array}$

$\delta_{\mathrm{h}} \quad$ Horizontaiverschiebung der oberen Betonplatte in Richtung der Querkraft

$\delta_{\mathrm{R}} \quad$ Randverschiebung der Wand 


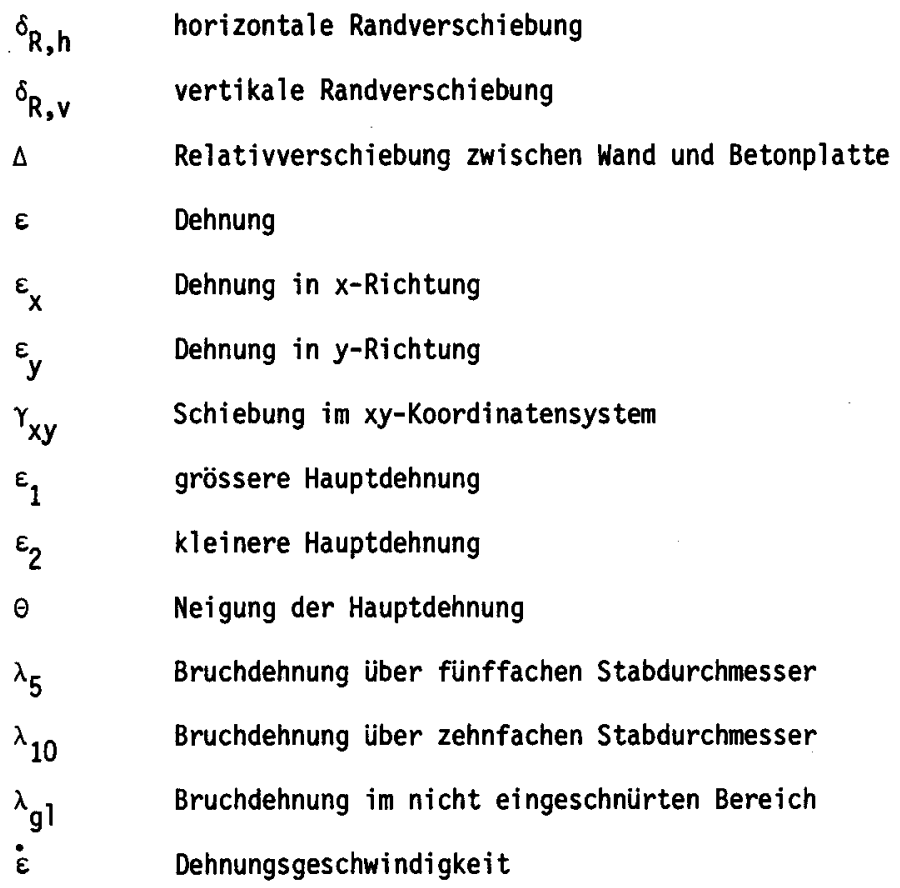

\section{Allgemeine Bezeichnungen}

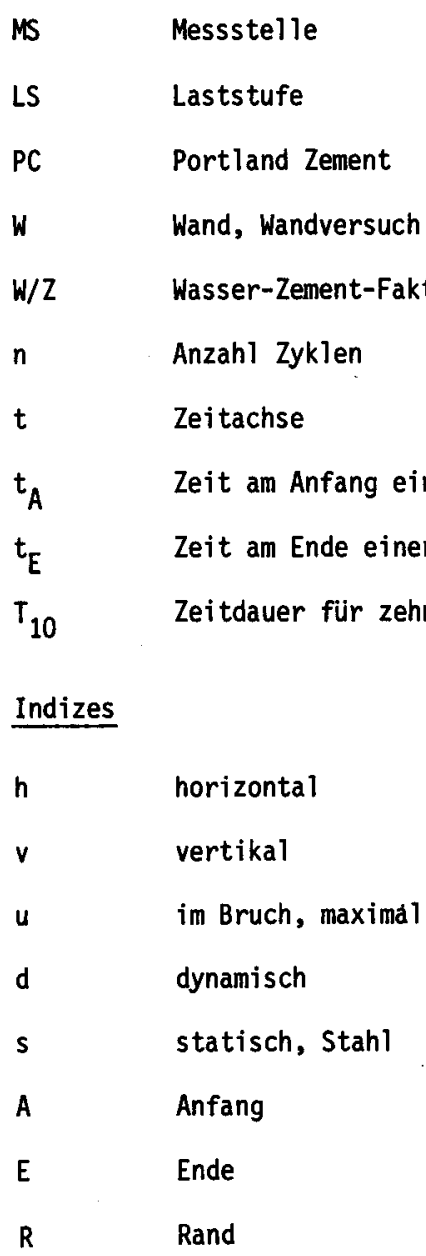




\section{Li teraturverzeichnis}

[1] H. Ganz, B. Thürlimann: "Versuche über die Festigkeit von zweiachsig beanspruchtem Mauerwerk", Institut für Baustatik und Konstruktion, ETH Zürich, Versuchsbericht Nr. 7502-3, Februar 1982, Birkhäuser Verlag Basel und Stuttgart.

[2] Schweizerischer Ingenieur- und Architekten-Verein (SIA): Norm SIA 177 "Mauerwerk", Ausgabe 1980 , Zürich.

[3] Schweizerischer Ingenieur- und Architekten-Verein (SIA): Empfehlung SIA 177/1 "Bemessung von Mauerwerkswänden unter Druckbeanspruchung", Ausgabe 1983, Zürich.

[4] Schweizerischer Ingenieur- und Architekten-Verein (SIA): Norm SIA 162 "Für die Berechnung, Konstruktion und Ausführung von Bauwerken aus Beton, Stahlbeton und Spannbeton", Ausgabe 1968, Zürich. 


\begin{tabular}{|l|c|c|c|c|c|c|}
\hline Versuch & $\begin{array}{c}\text { Normalkraft } \\
\text { N } \\
{[\mathrm{kN}]}\end{array}$ & $\begin{array}{c}\text { Exzentrizität } \\
\text { von N in Wand- } \\
\text { ebene } \\
{[\mathrm{mm}]}\end{array}$ & $\begin{array}{l}\text { Lagerfugen- } \\
\text { bewehrung }\end{array}$ & $\begin{array}{l}\text { Vertikale } \\
\text { Vorspannung } \\
\mathrm{P}_{0} \\
{[\mathrm{kN}]}\end{array}$ & Belastung & Prijfalter \\
\hline W1 & -415 & 0 & - & - & progressiv & 21 \\
W2 & -1287 & 0 & - & - & progressiv & 19 \\
W3 & -415 & 0 & $2 \phi 5$ & - & progressiv & 20 \\
W4 & -423 & 840 & - & - & progressiv & 21 \\
W5 & -424 & 1720 & $2 \phi 5$ & 277 & progressiv & 21 \\
W6 & -418 & 0 & - & - & zyklisch & 16 \\
W7 & -1290 & 0 & - & - & zyklisch & 22 \\
\hline
\end{tabular}

Tabelle 1: Versuchsprogramm

\begin{tabular}{|c|c|c|}
\hline 1. Format & Breite/Länge/Höhe [mm] & $150 / 300 / 190$ \\
\hline \multirow[t]{2}{*}{ 2. Lochung } & Lochfläche $\left[\mathrm{mm}^{2}\right]$ & $20 \cdot 200$ \\
\hline & Lochfläche [\%] der Bruttofläche & 46 \\
\hline 3. Steinmasse & Mittelwert $[\mathrm{kg}]$ & 7.22 \\
\hline 4. Dichte & Mittelwert $\left[\mathrm{kg} / \mathrm{m}^{3}\right]$ & 854 \\
\hline 5. Spez. Saugfähigkeit & Mittelwert $\left[\mathrm{g} / \mathrm{dm}^{2} \cdot \min \right]$ & 26 \\
\hline 6. Steindruckfestigkeit & Mittelwert $\left[\mathrm{N} / \mathrm{mm}^{2}\right]$ & 37.4 \\
\hline 7. Querzugfestigkeit & Mittelwert $\left[\mathrm{N} / \mathrm{mm}^{2}\right]$ & 9.1 \\
\hline
\end{tabular}

Tabelle 2: Resultate der Normprüfungen des Backsteins

\begin{tabular}{|l|c|c|c|c|c|c|}
\hline $\begin{array}{l}\text { Mörtel } \\
\text { Zu Versuch }\end{array}$ & $\begin{array}{l}\text { Alter bei } \\
\text { Prüfung } \\
{[\text { in Tagen] }}\end{array}$ & $\begin{array}{c}\text { Dosierung } \\
\mathrm{PC} \\
{\left[\mathrm{kg} / \mathrm{m}^{3}\right]}\end{array}$ & $\begin{array}{l}\text { Dichte des } \\
\text { frischen } \\
\text { Mörtels } \\
{\left[\mathrm{kg} / \mathrm{m}^{3}\right]}\end{array}$ & $\begin{array}{l}\text { Dichte des } \\
\text { erhärteten } \\
\text { Mörtels } \\
{\left[\mathrm{kg} / \mathrm{m}^{3}\right]}\end{array}$ & $\begin{array}{l}\text { Biegezug- } \\
\text { festigkeit } \\
{\left[\mathrm{N} / \mathrm{mm}^{2}\right]}\end{array}$ & $\begin{array}{c}\text { Druck- } \\
\text { festigkeit } \\
{\left[\mathrm{N} / \mathrm{mm}^{2}\right]}\end{array}$ \\
\hline W1 & 25 & 363 & 2160 & 2149 & 5.5 & 23.9 \\
W2 & 30 & 365 & 2171 & 2138 & 6.3 & 23.8 \\
W3 & 29 & 361 & 2149 & 2158 & 7.4 & 31.1 \\
W4 & 29 & 361 & 2154 & 2188 & 7.7 & 33.8 \\
W5 & 34 & 358 & 2134 & 2184 & 7.0 & 27.6 \\
W6 & 24 & 363 & 2164 & 2211 & 7.4 & 28.2 \\
W7 & 34 & 363 & 2162 & 2207 & 7.5 & 31.2 \\
\hline
\end{tabular}

Tabelle 3: Resultate der Prüfung von Mörtelprismen $W / Z \simeq 0.79 ;$ Wasserzusatz $\simeq 13.3 \%$; Mittelwerte aus je 6 Prismen 


\begin{tabular}{|c|c|c|c|c|c|c|c|c|c|}
\hline $\begin{array}{l}\text { Nenndurchmesser } \\
{[\mathrm{mm}]}\end{array}$ & $\begin{array}{l}\text { Aeff } \\
{\left[\mathrm{mm}^{2}\right]}\end{array}$ & $\begin{array}{c}f_{y, s} \\
{\left[N / \mathrm{mm}^{2}\right]}\end{array}$ & $\begin{array}{c}f_{y, d} \\
{\left[N / m^{2}\right]}\end{array}$ & $\begin{array}{c}f_{s t, s} \\
{\left[N / \mathrm{mm}^{2}\right]}\end{array}$ & $\begin{array}{c}f_{s t, d} \\
{\left[N / m^{2}\right]}\end{array}$ & $\lambda_{5}$ & $\lambda_{10}$ & $\lambda_{\mathrm{gl}}$ & $\begin{array}{l}\text { Steifigkeit } \\
{\left[\mathrm{kN} / \mathrm{mm}^{2}\right]}\end{array}$ \\
\hline 1) & 20.05 & 506.3 & 524.6 & 545.2 & 570.3 & 0.142 & 0.077 & 0.014 & 196.2 \\
\hline 2) & 112.72 & - & 1425 & - & 1647 & - & 0.068 & - & - \\
\hline
\end{tabular}

Tabelle 4: Festigkeitswerte der Bewehrung

1) Lagerfugenbewehrung; Verwendung $W 3$, W5; Mittel aus 6 Proben

2) Vorspannstah1; Verwendung W5; Mittel aus 16 Proben 

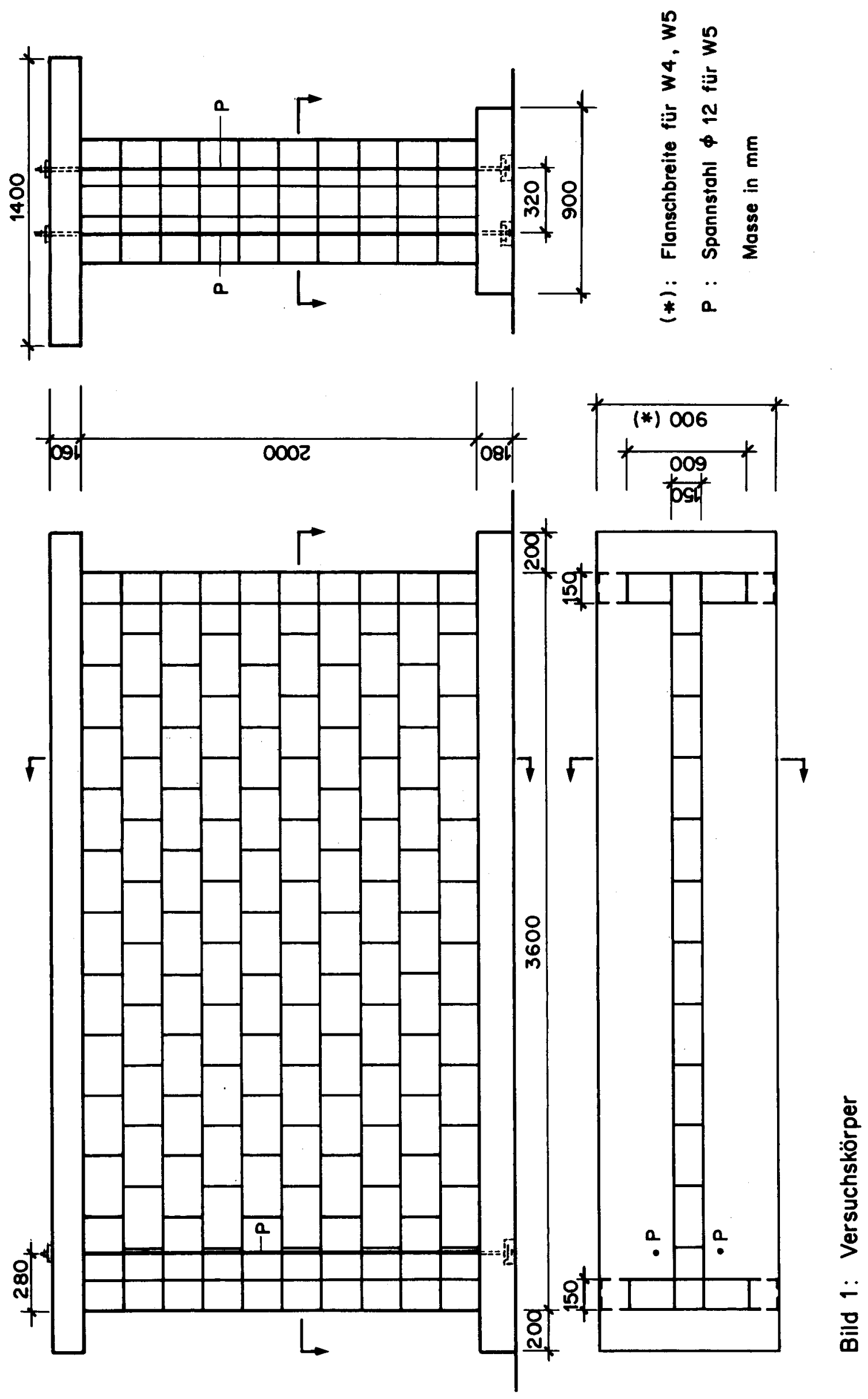

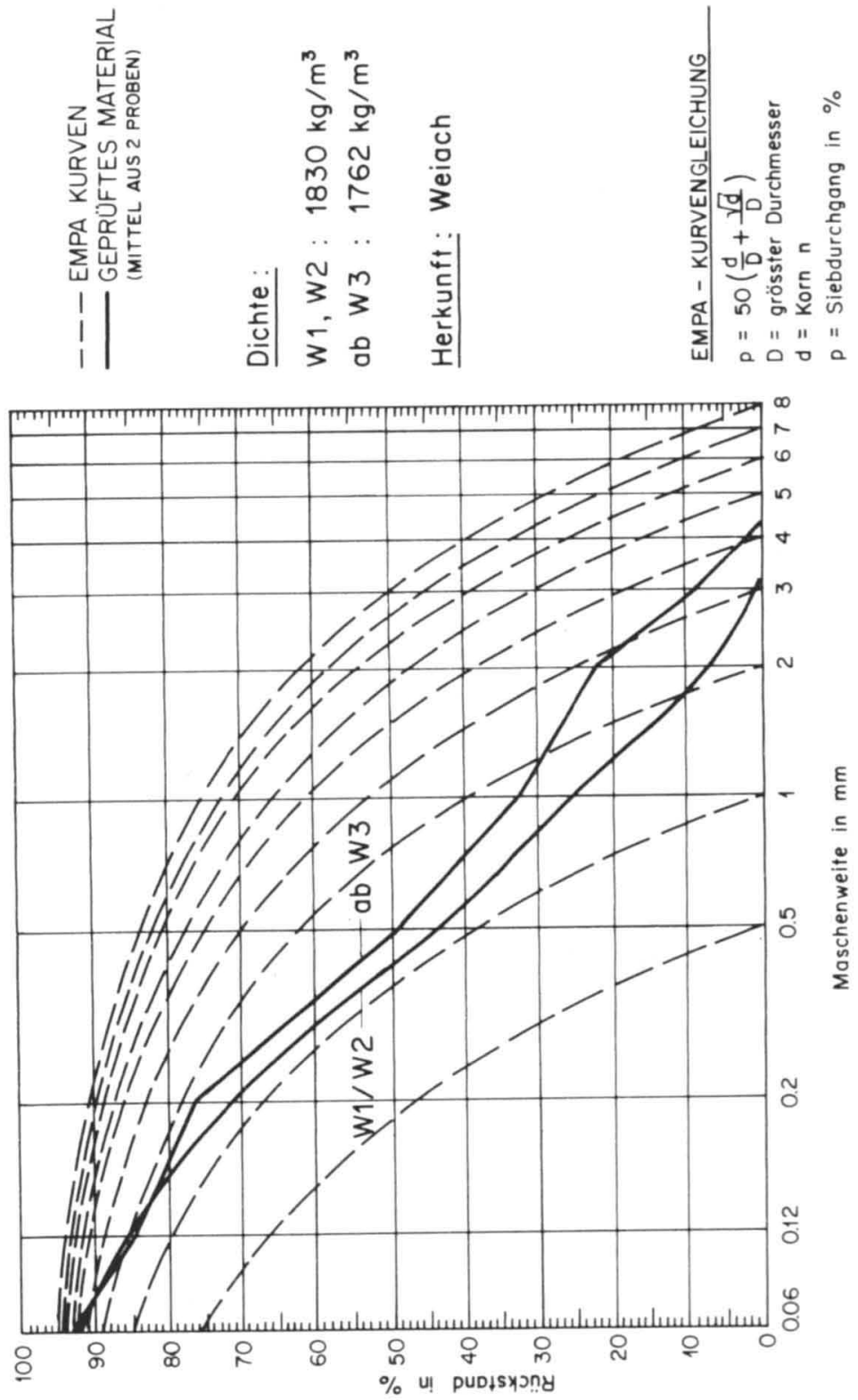

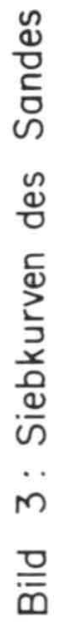

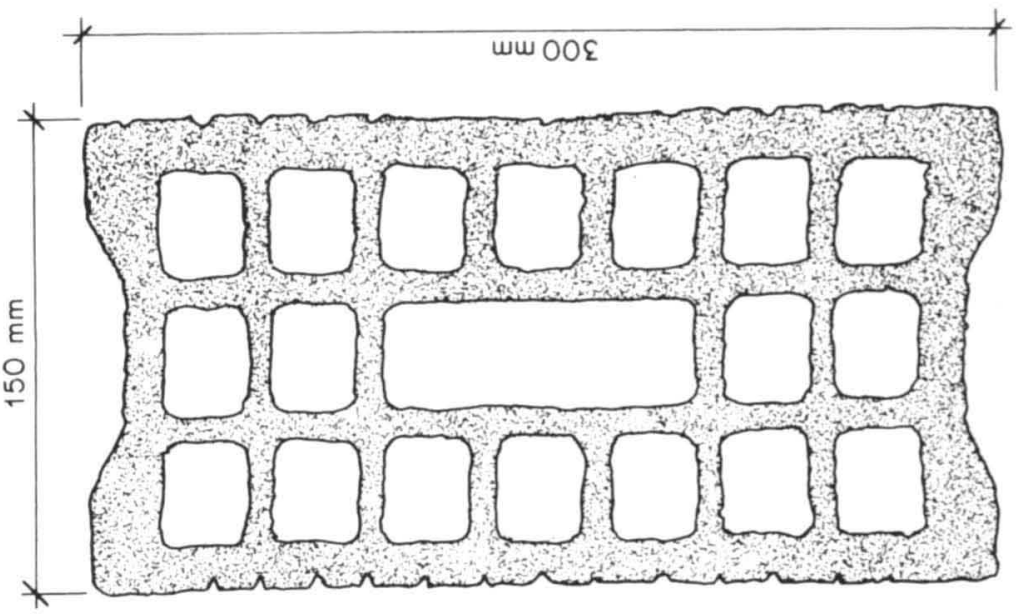

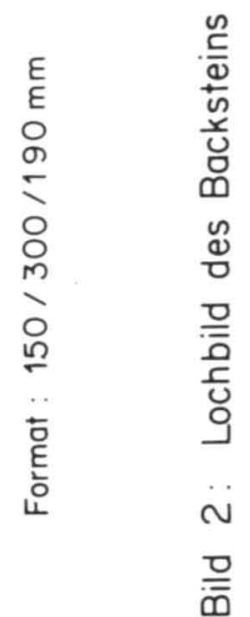




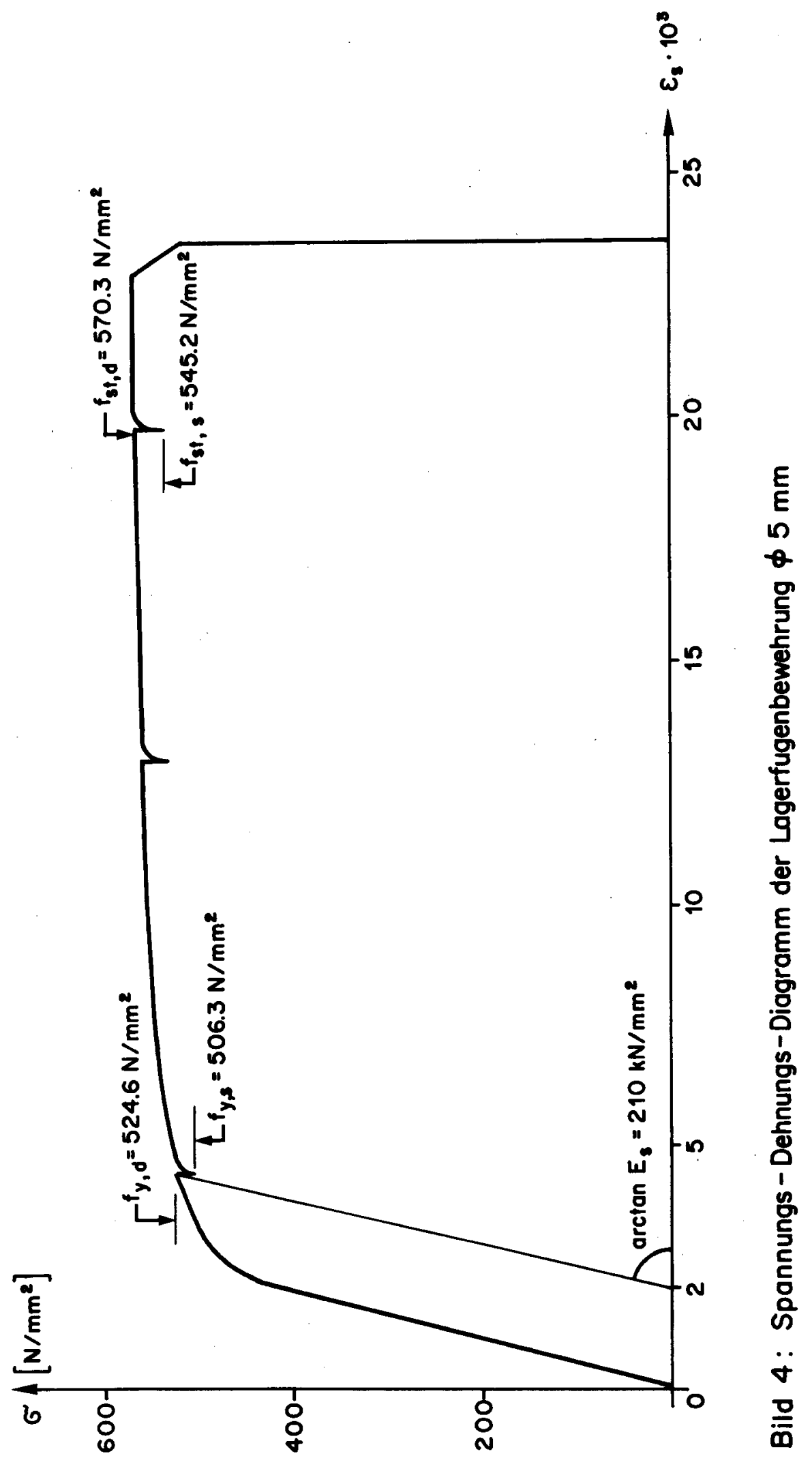




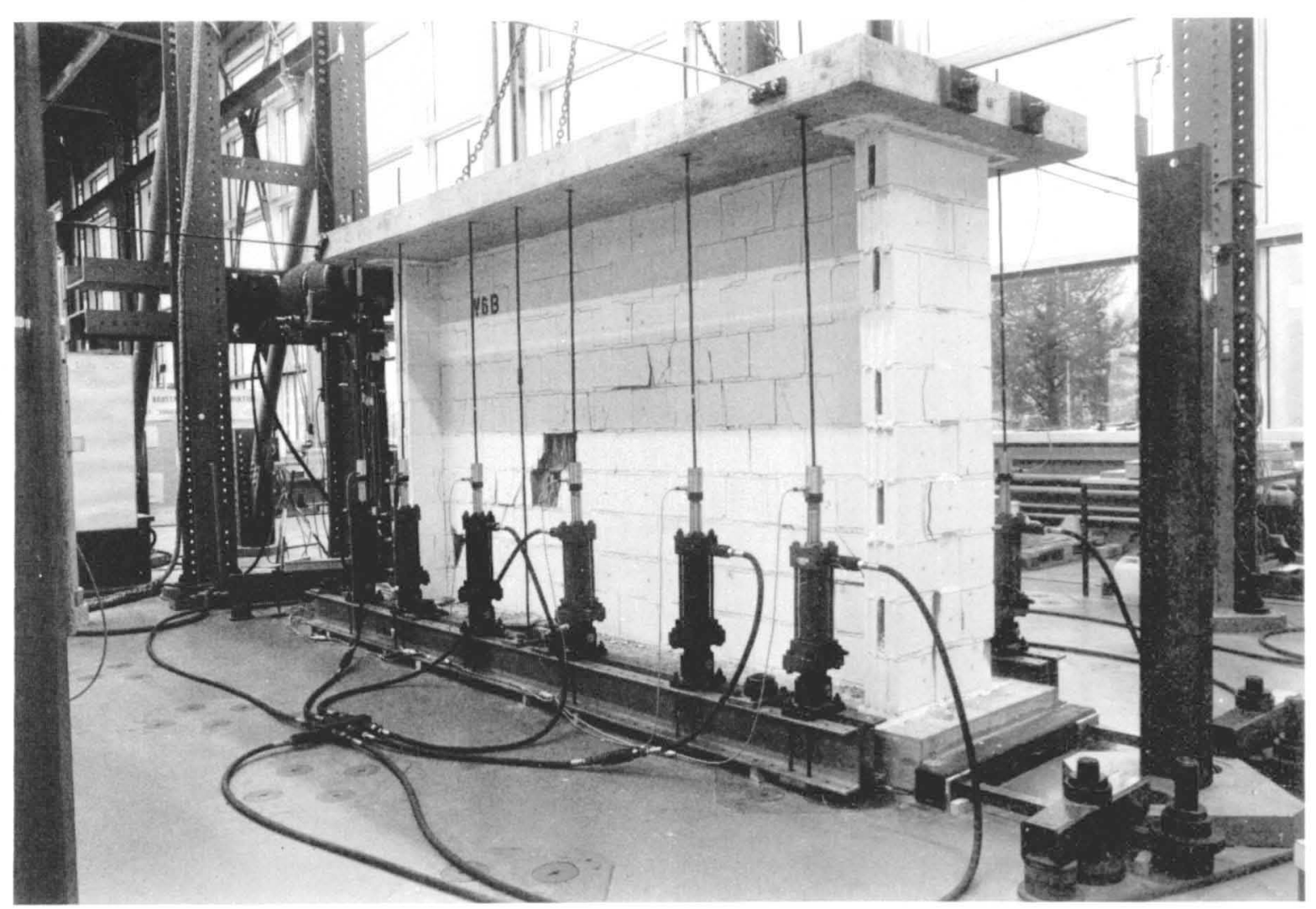

Bild 5: Versuchsanlage für zentrische Normalkraft

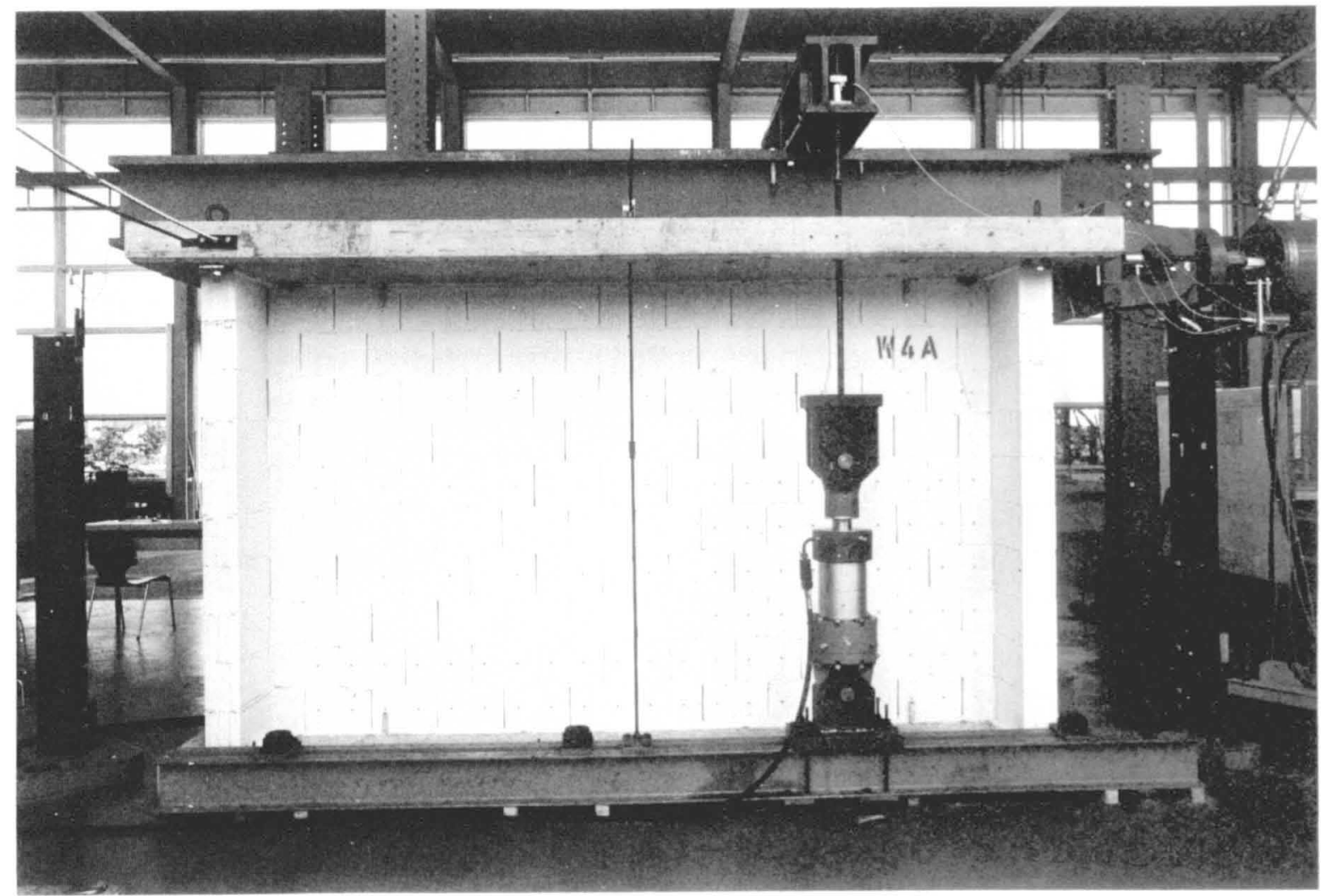

Bild 6: Versuchsanlage für exzentrische Normalkraft 

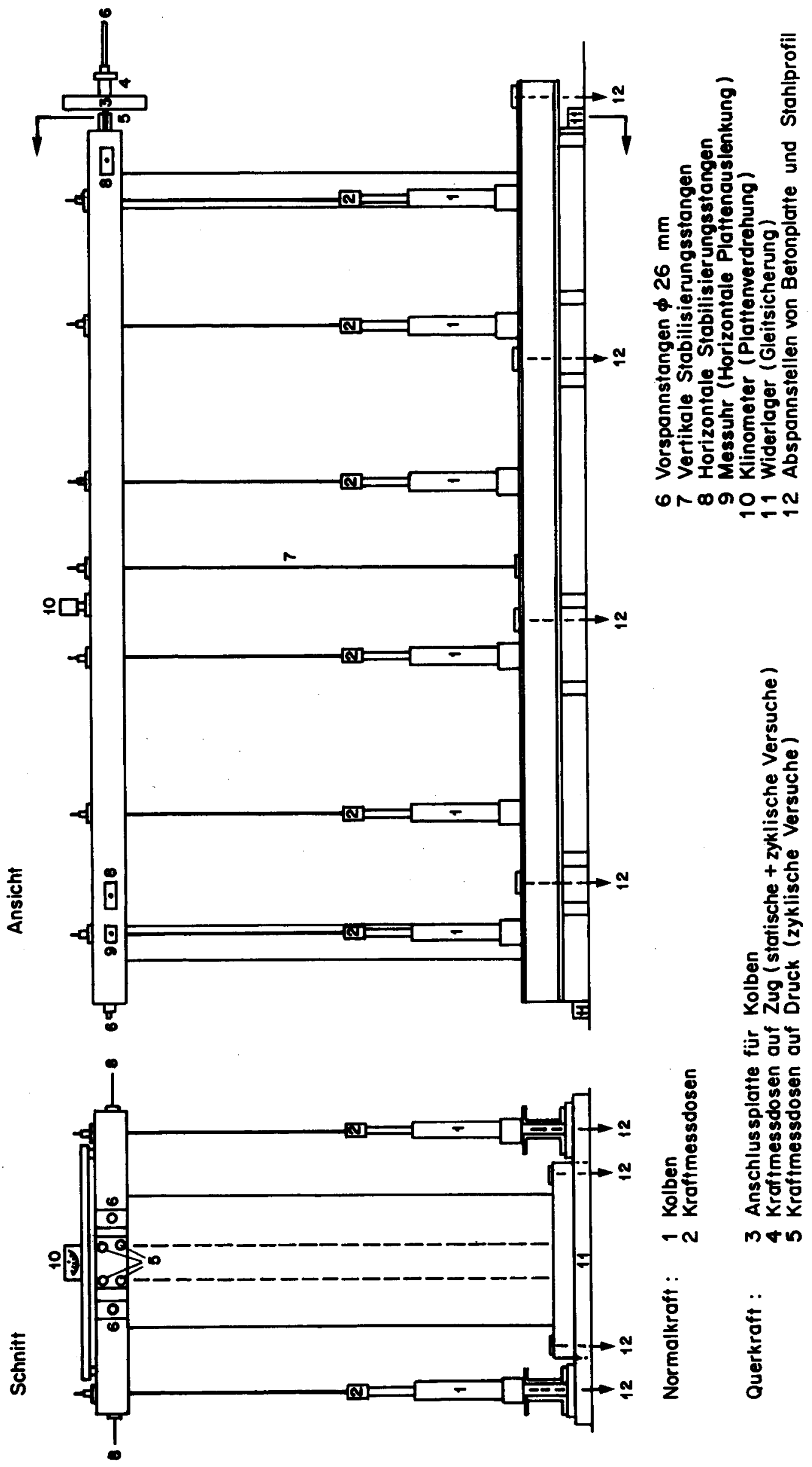

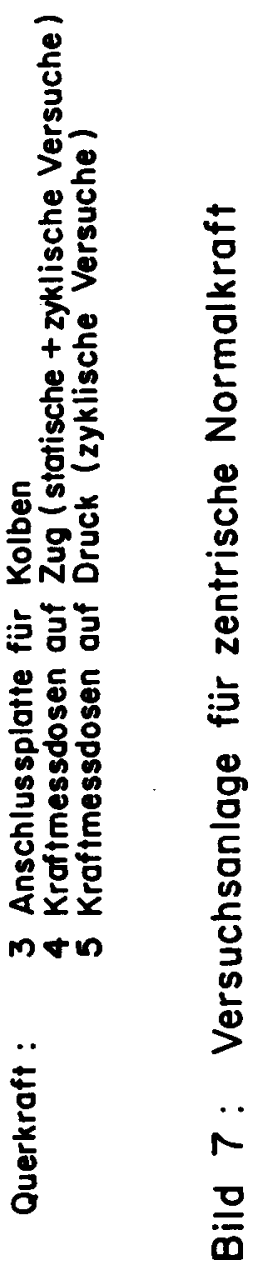




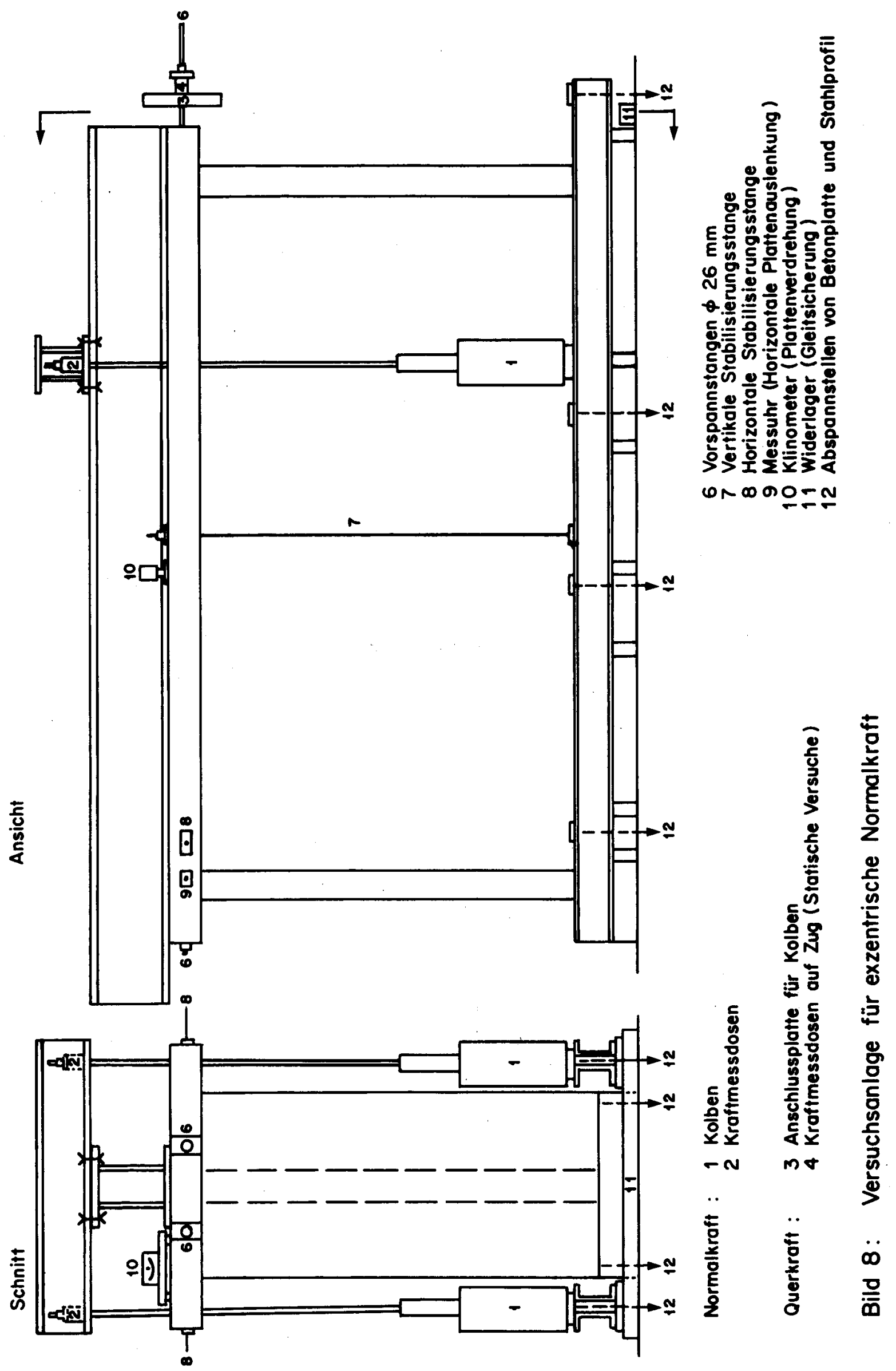



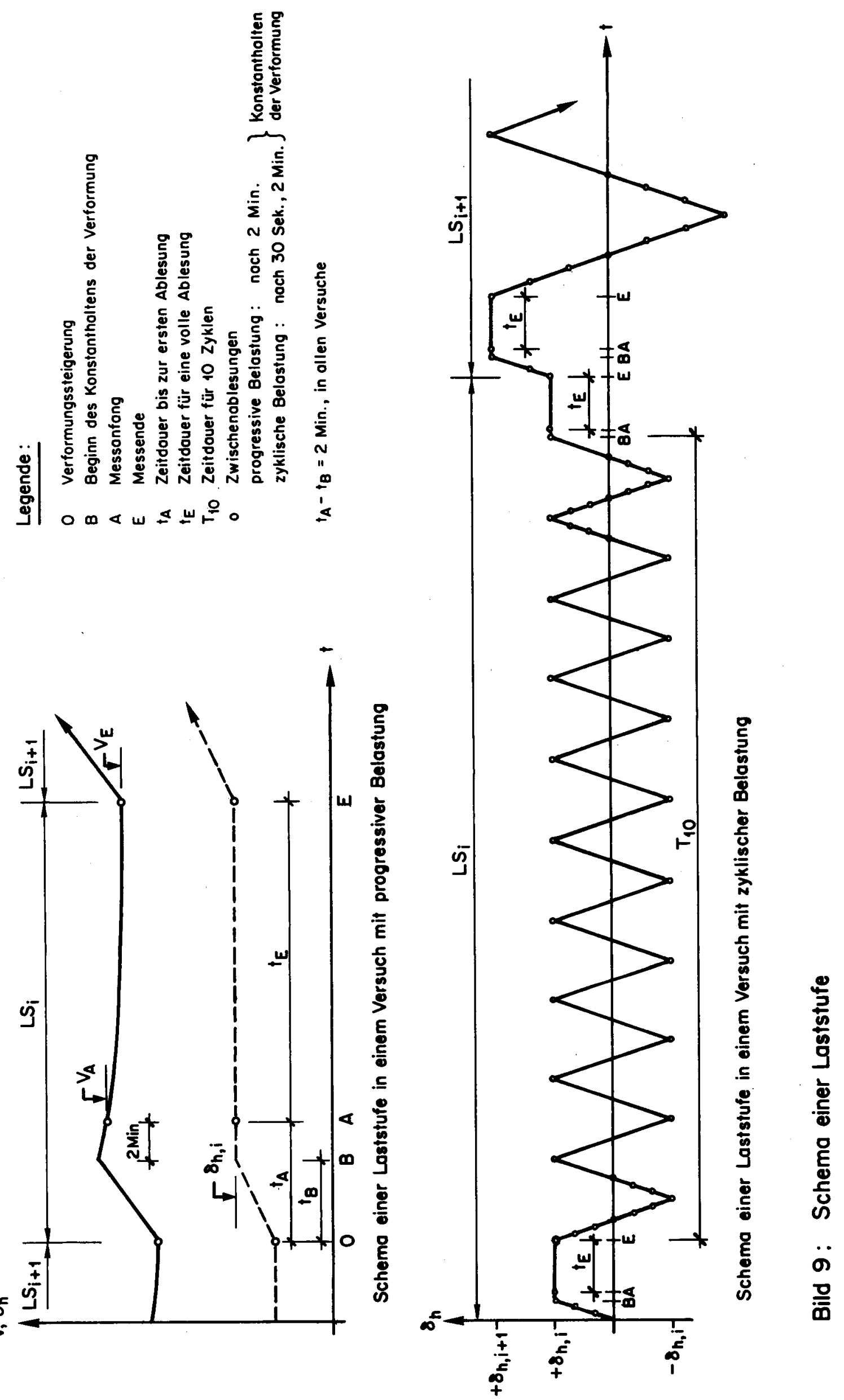


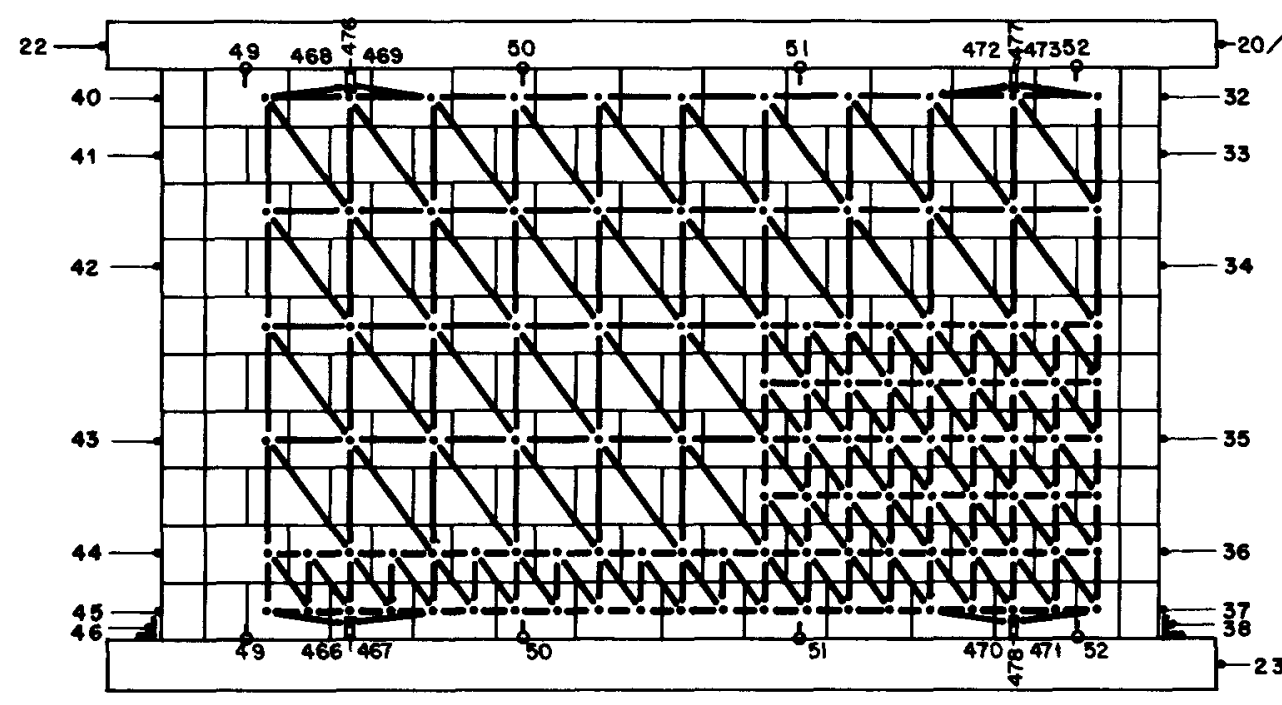

Seite A

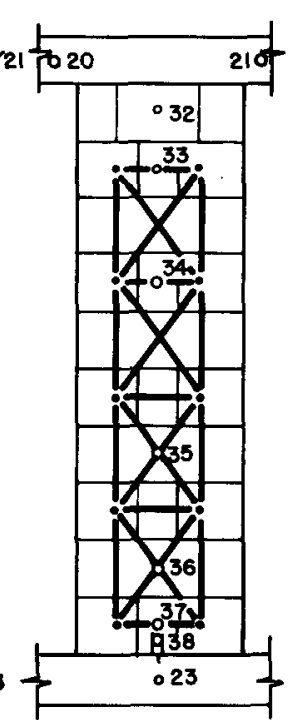

Flansch R

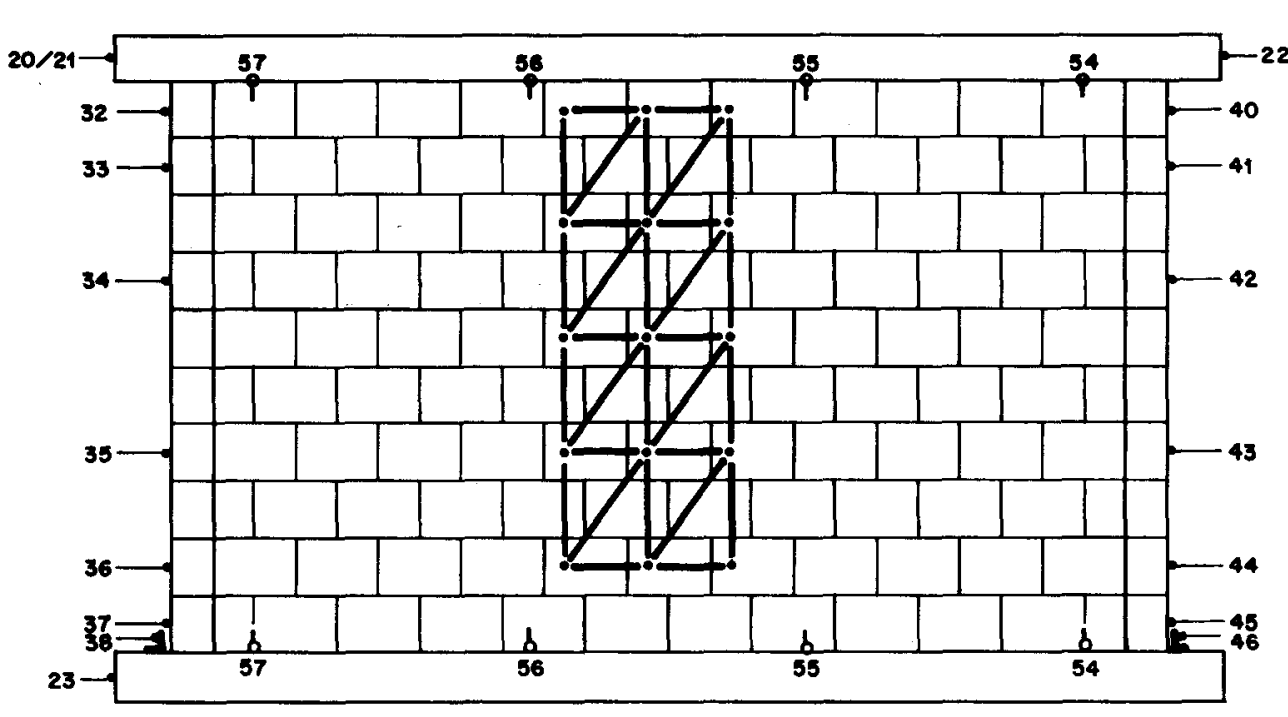

Seite B

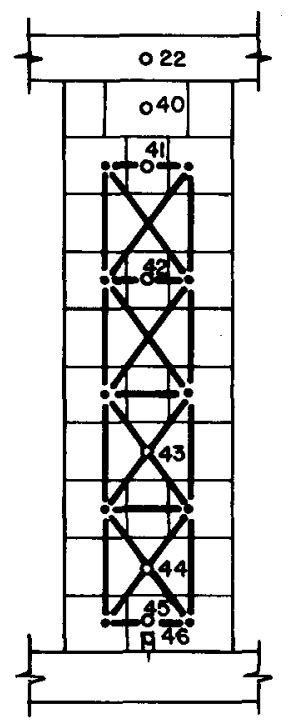

Flansch L
Horizontalverschiebung der Betonplatten:

$20,21,22,23$

38,46

Induktive Wegaufnehmer

Induktive Messstangen

Scheiben - Randverschiebungen:

-vertikal :

$49 \div 57$

-horizontal :

$32 \div 46$

Induktive Messstangen

Induktive Messstangen
Knotenverschiebungen:

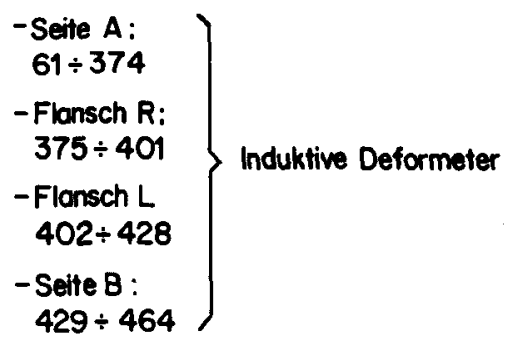

Relativverschiebung Wand-Betonplatten : $465 \div 479$ Induktive Deformeter

Bild 10 : Messnetzplan 

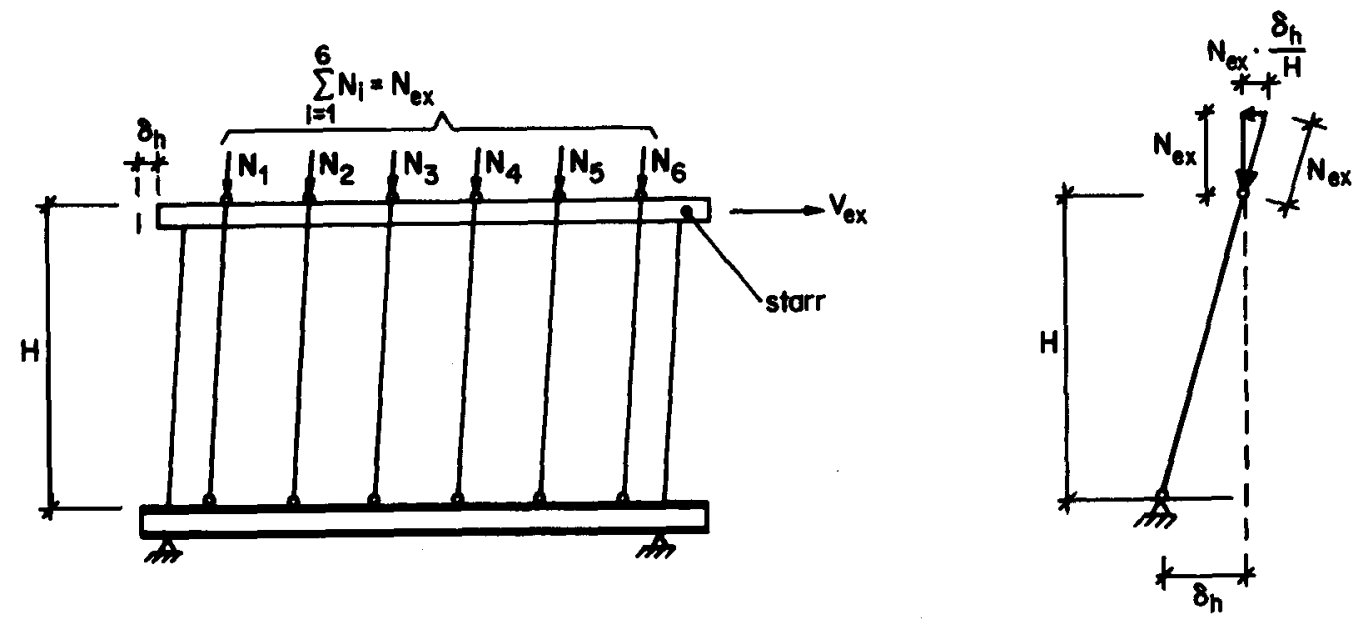

Bild 11 : Bestimmung der Querkraft

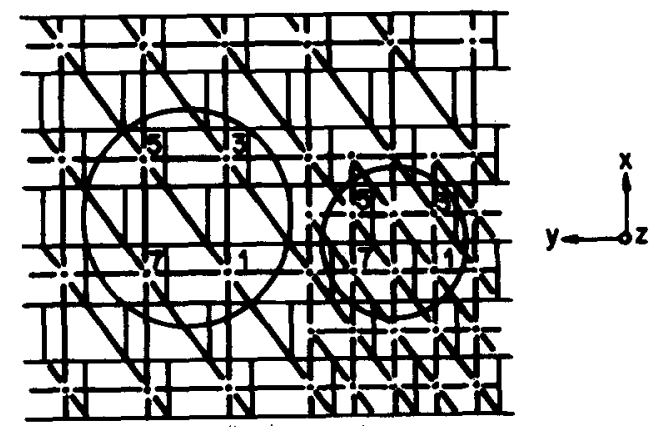

Messnetz

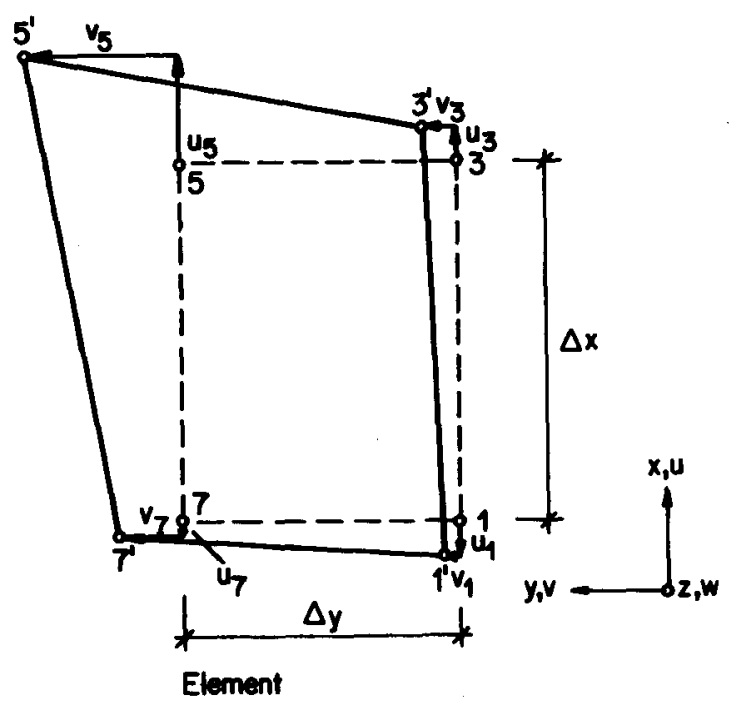

Durchschnittiche Verzerrungen eines Elementes:

$$
\begin{aligned}
& \varepsilon_{x}=\frac{1}{2}\left\{\left(u_{5} u_{7}\right)+\left(u_{3}-u_{1}\right)\right\} \cdot \frac{1}{\Delta x} \\
& \varepsilon_{y}=\frac{1}{2}\left\{\left(v_{5}-v_{3}\right)+\left(v_{7}-v_{1}\right)\right\} \cdot \frac{1}{\Delta y} \\
& \gamma_{x y}=\frac{1}{2}\left\{\left(u_{5}-u_{3}\right)+\left(u_{7}-u_{1}\right)\right\} \cdot \frac{1}{\Delta y}+\frac{1}{2}\left\{\left(v_{5}-v_{7}\right)+\left(v_{3}-v_{1}\right)\right\}-\frac{1}{\Delta x} \\
& \varepsilon_{1}=\frac{1}{2}\left\{\left(\varepsilon_{x}+\varepsilon_{y}\right)+\sqrt{\left(\varepsilon_{x}-\varepsilon_{y}\right)^{2}+\gamma_{y}^{2}}\right\} \\
& \varepsilon_{2}=\frac{1}{2}\left\{\left(\varepsilon_{x}+\varepsilon_{y}\right)-\sqrt{\left(\varepsilon_{x}-\varepsilon_{y}\right)^{2}+\gamma_{x y}^{2}}\right\} \\
& \text { e }=\frac{1}{2} \arctan \left(\frac{\gamma_{x y}}{\varepsilon_{x}-\varepsilon_{y}}\right)
\end{aligned}
$$

Bild 12 : Berechnung der Verzerrungen 


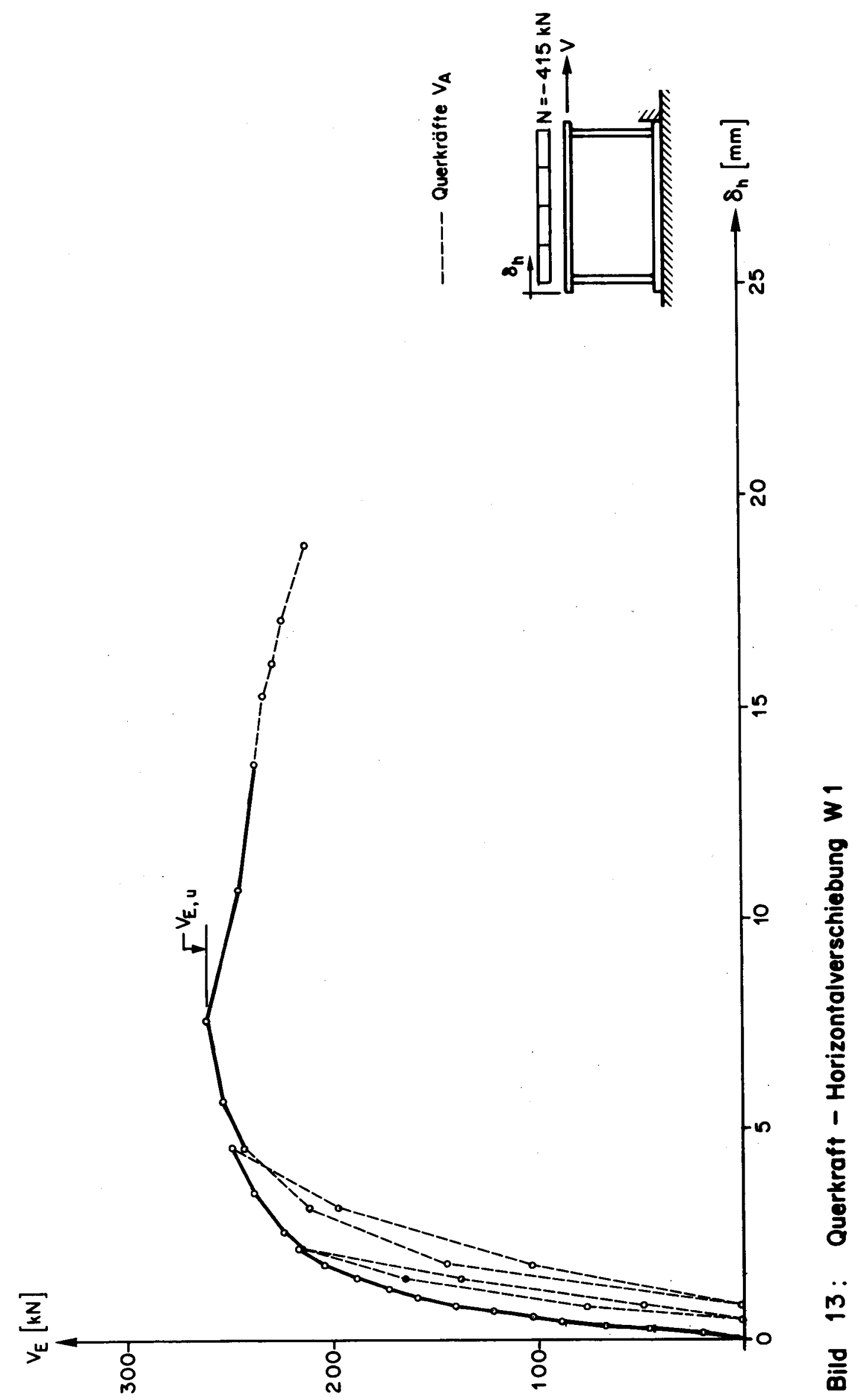




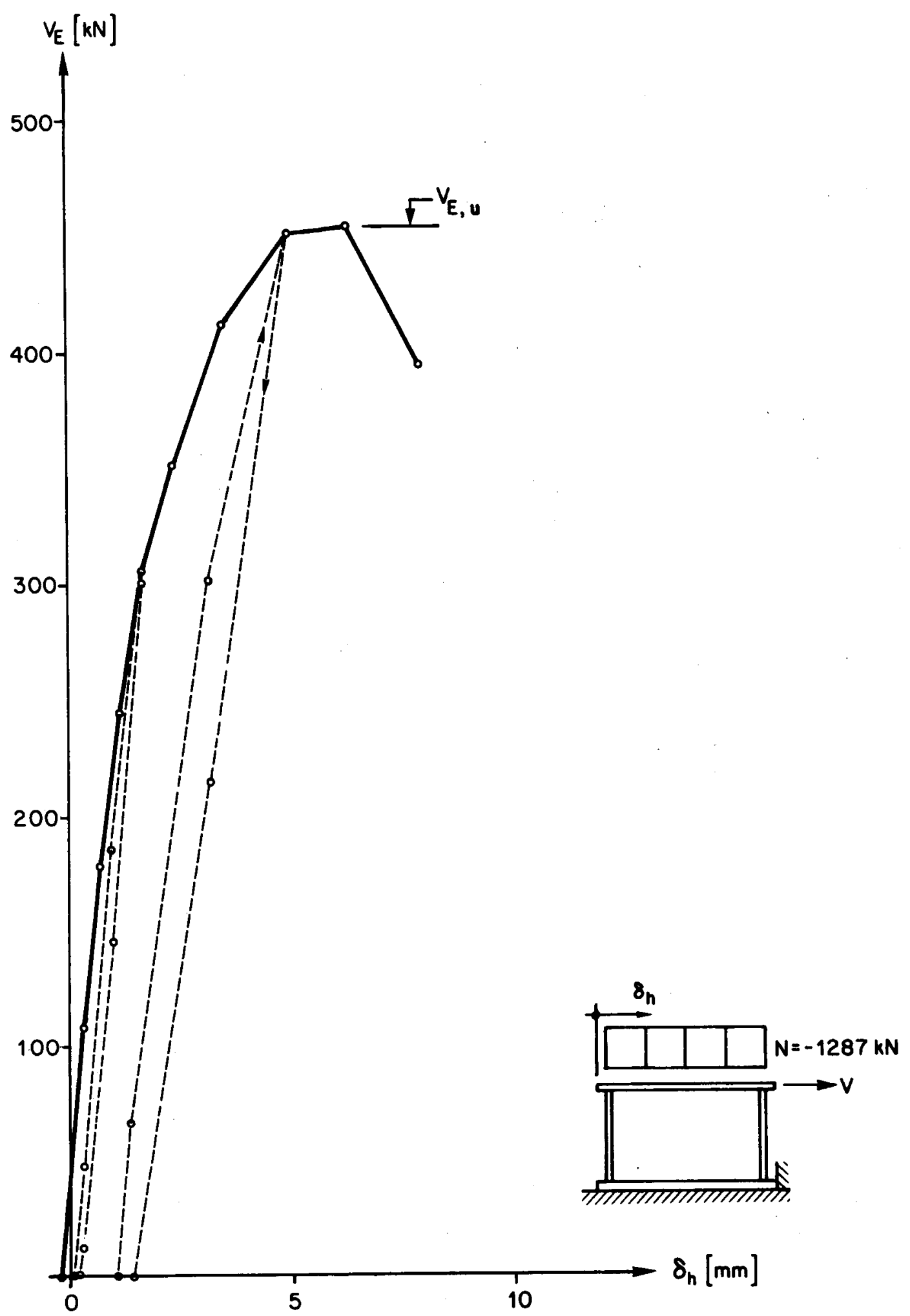

Bild 14: Querkraft - Horizontalverschiebung W2 


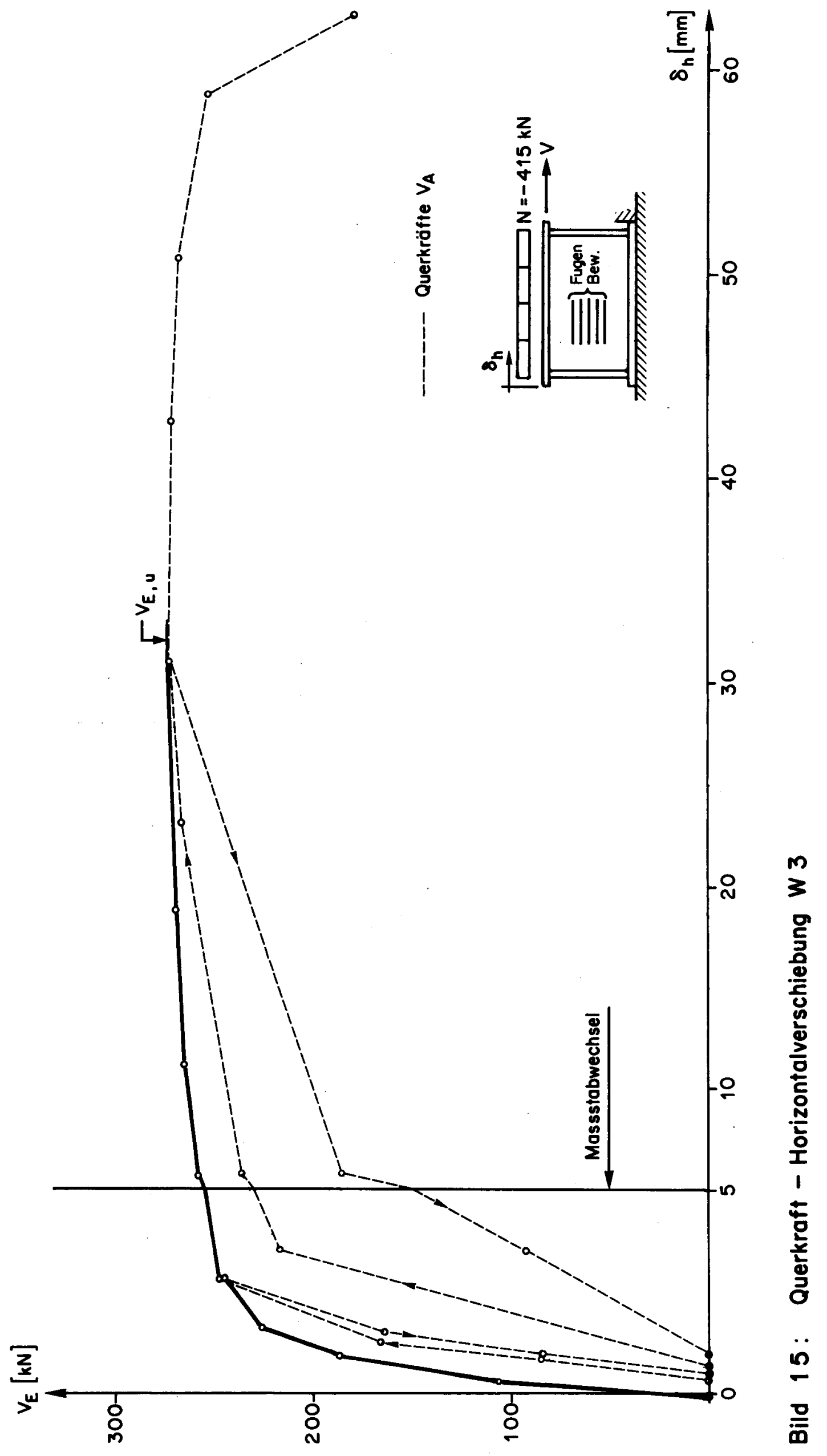




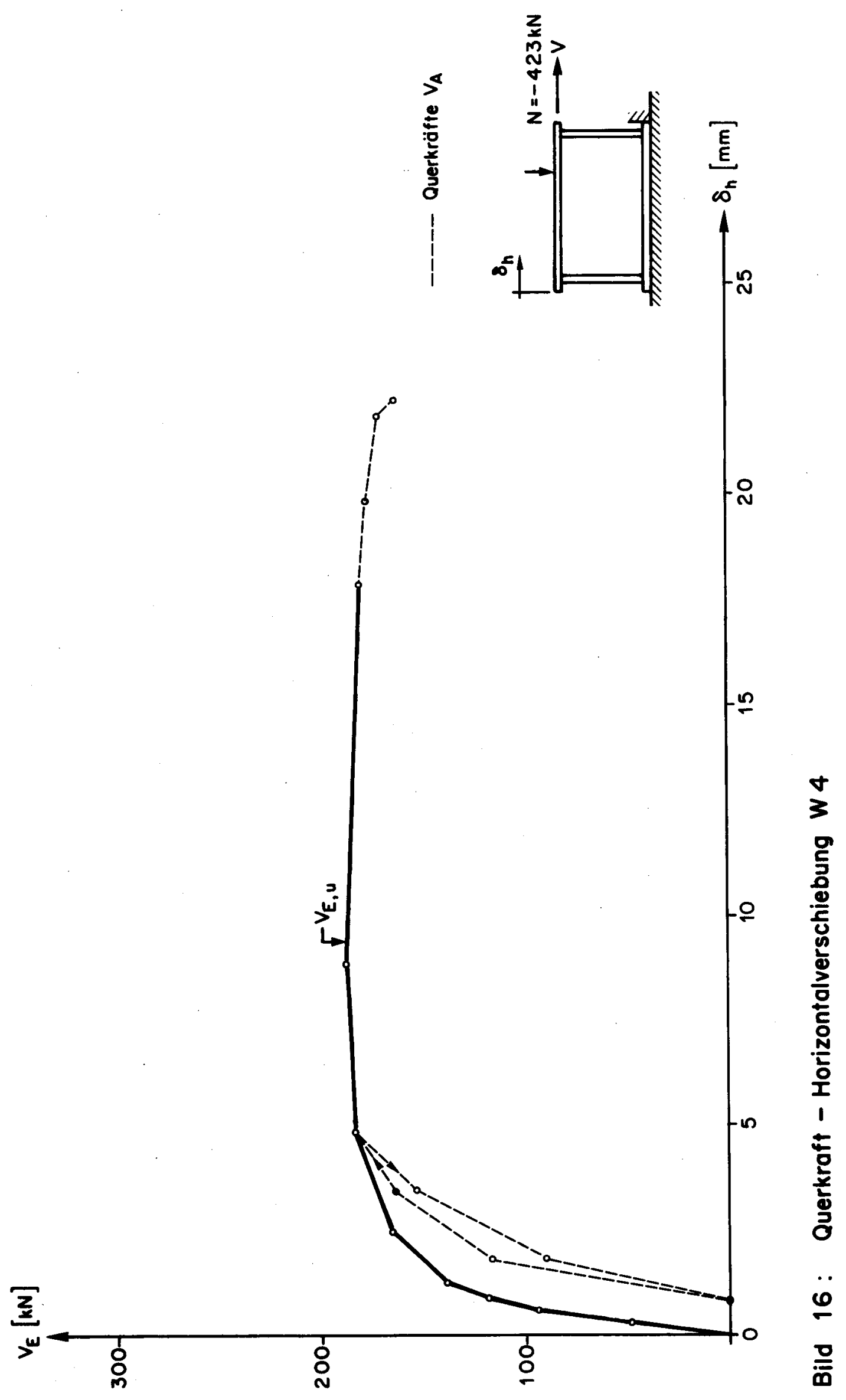




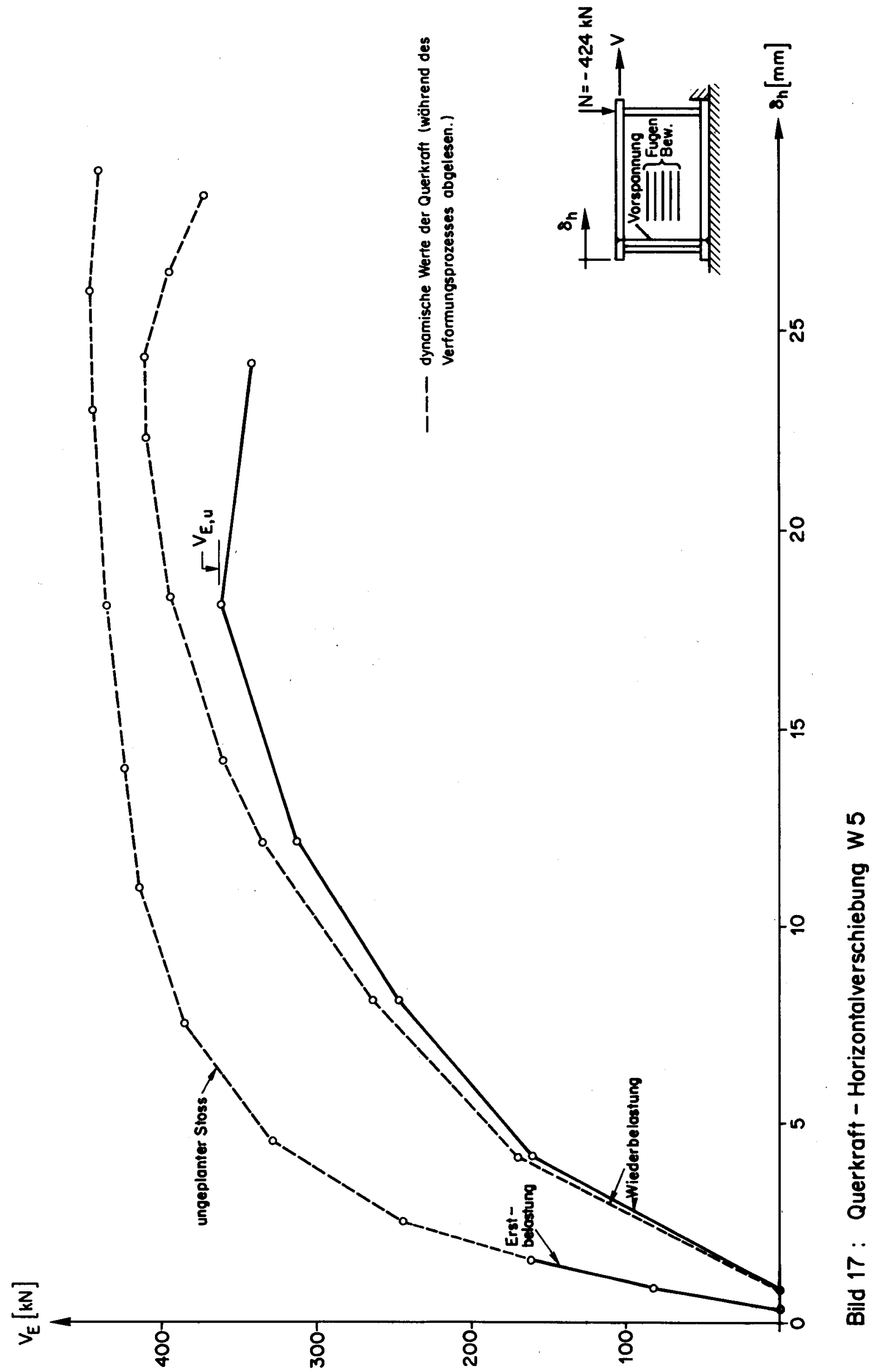




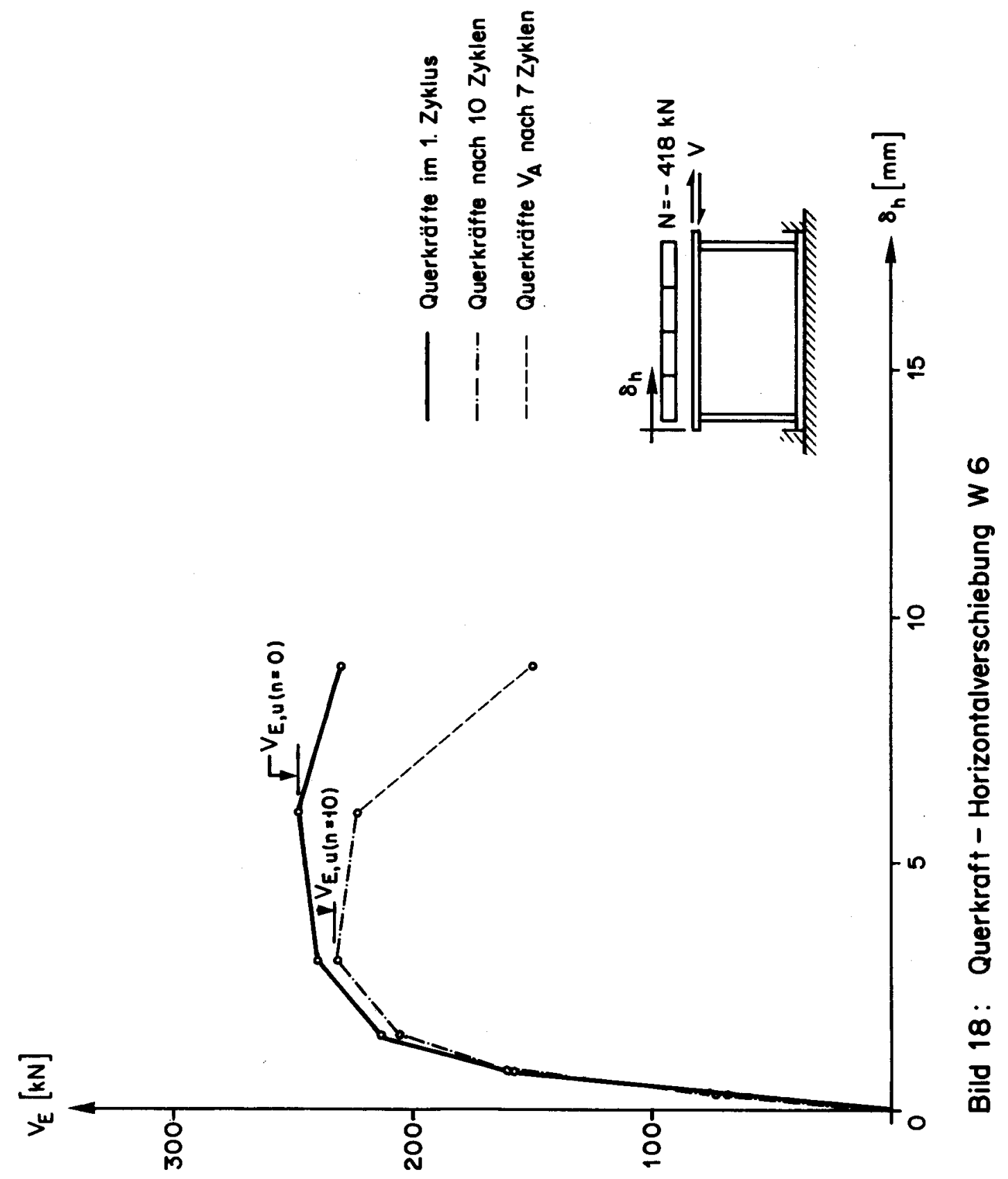




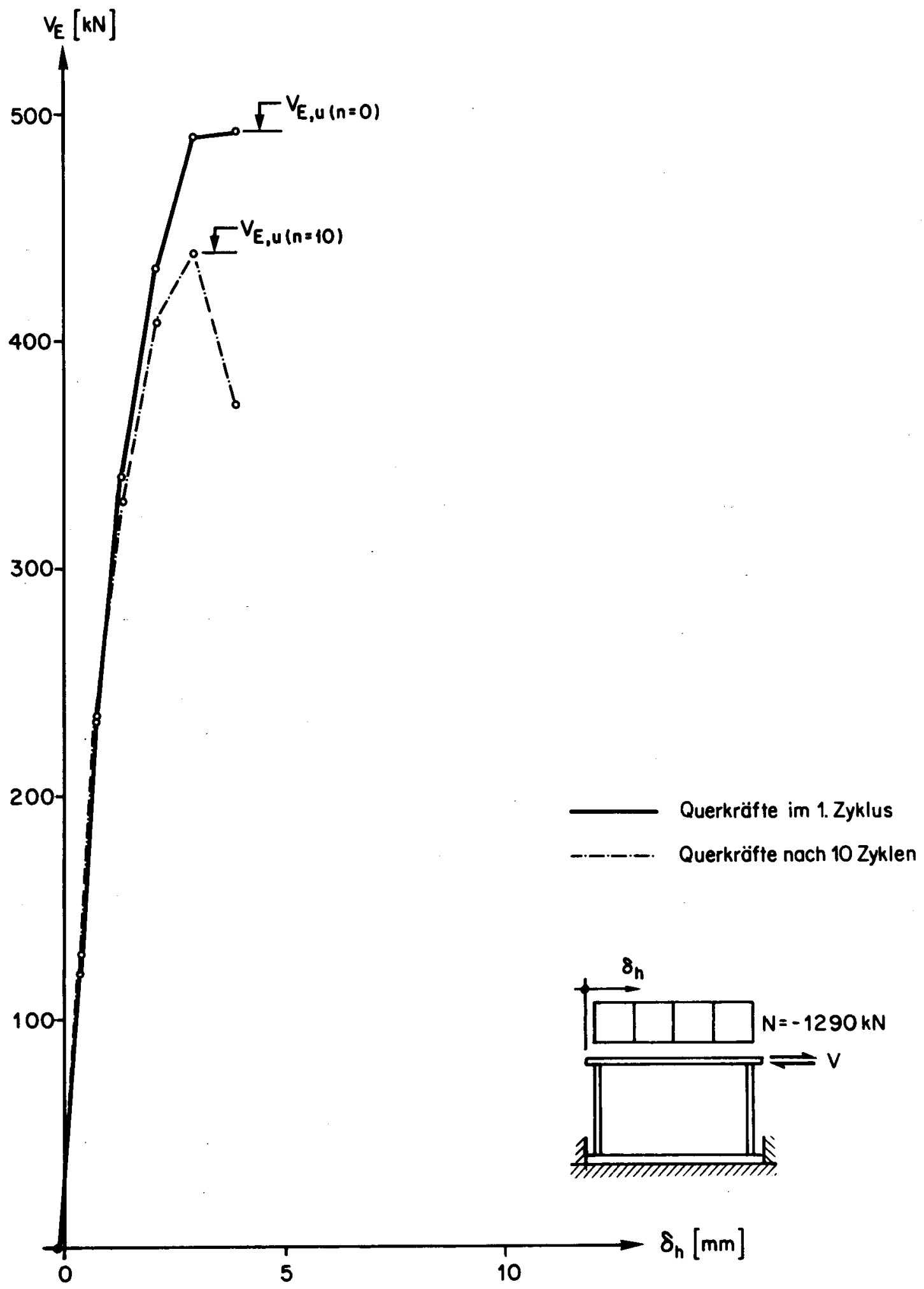

Bild 19: Querkraft - Horizontalverschiebung W7 

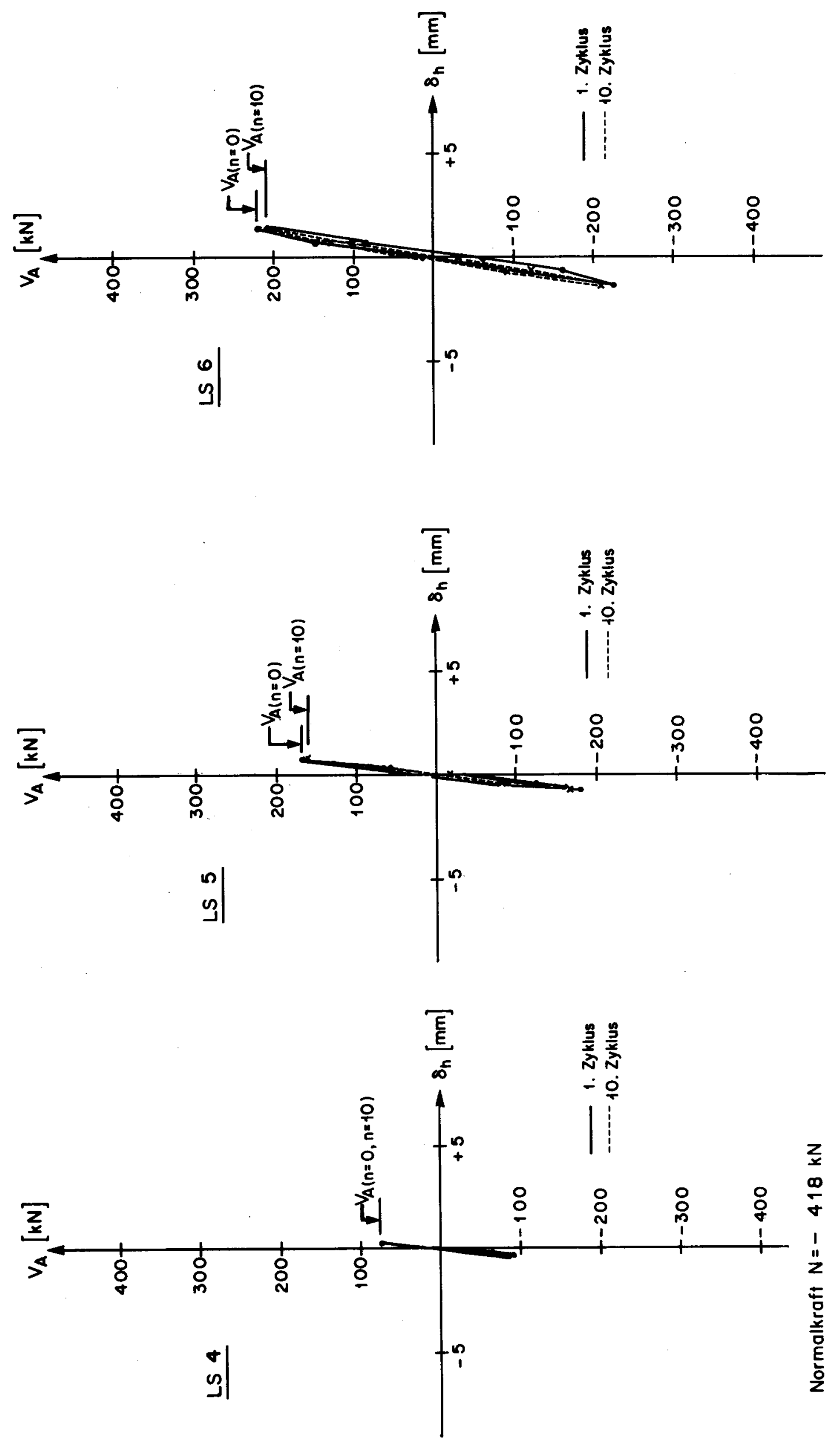

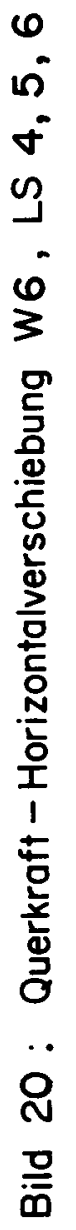



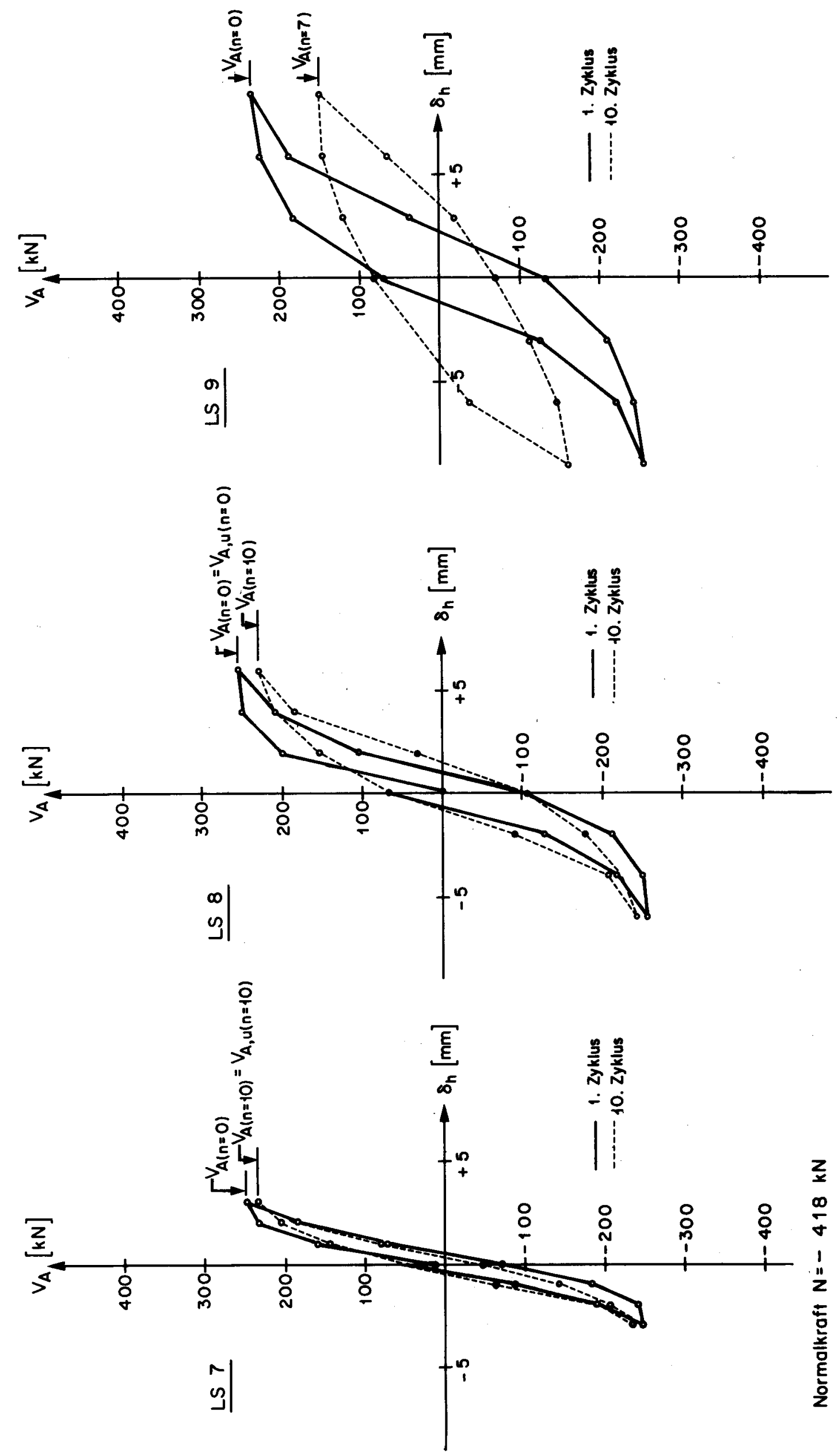

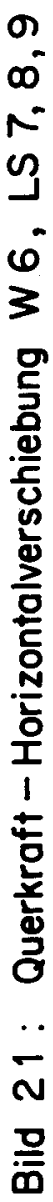



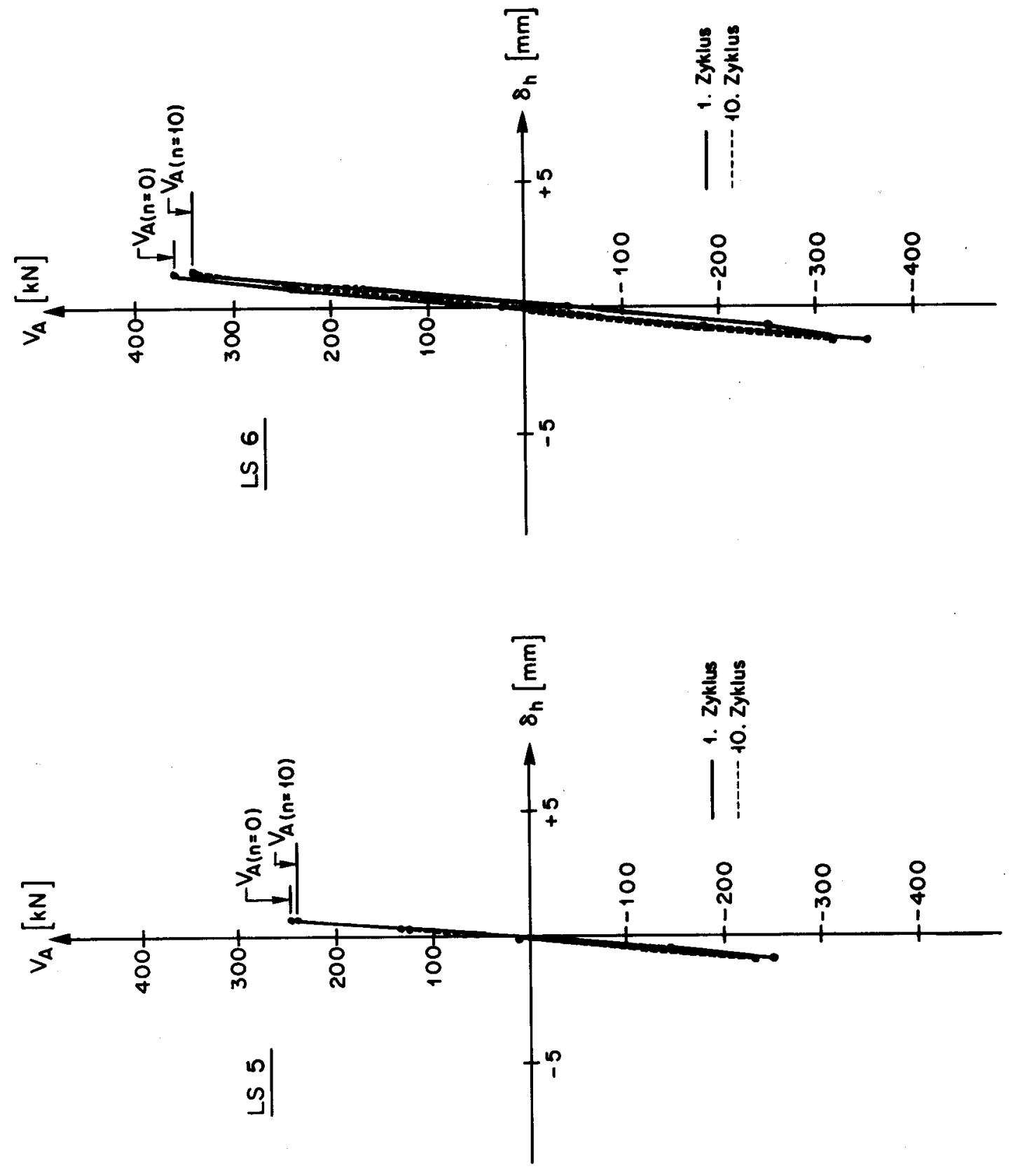

0
0
0

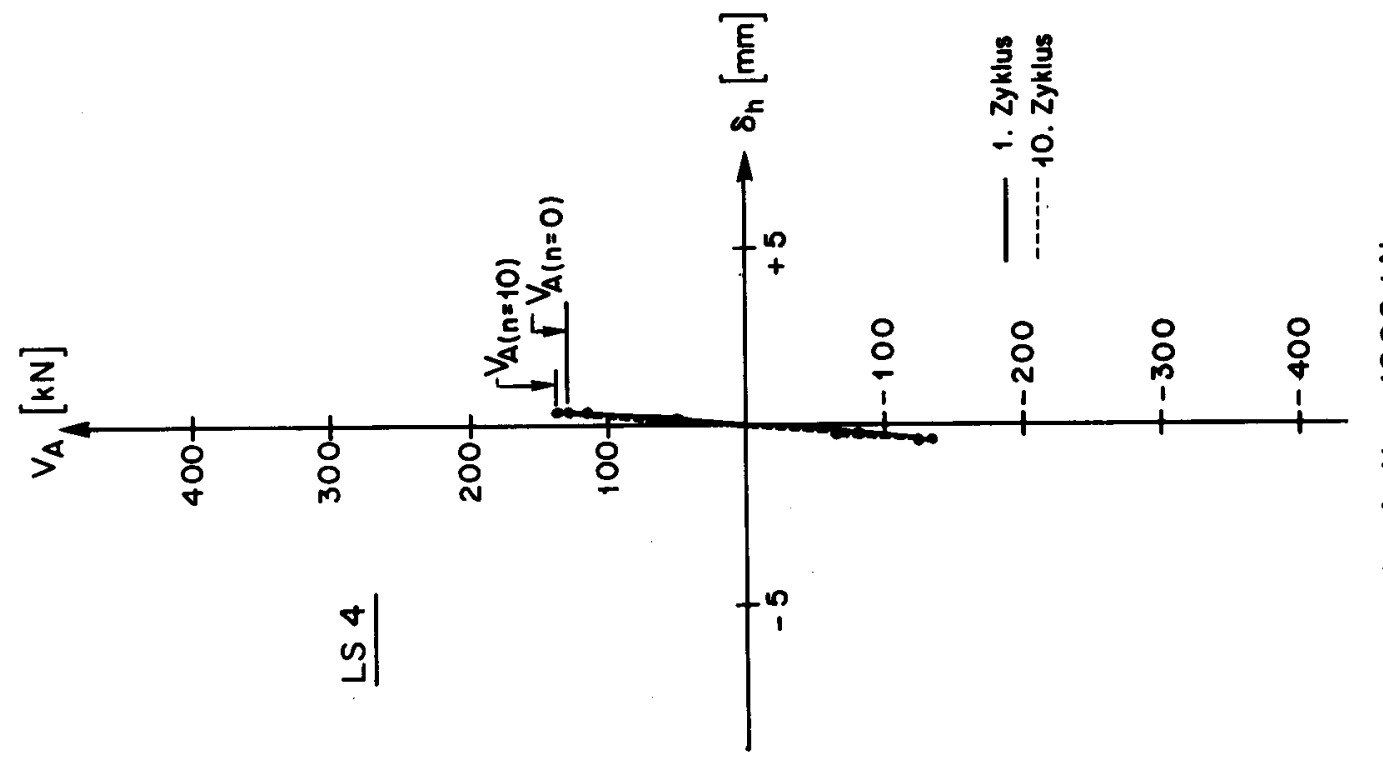



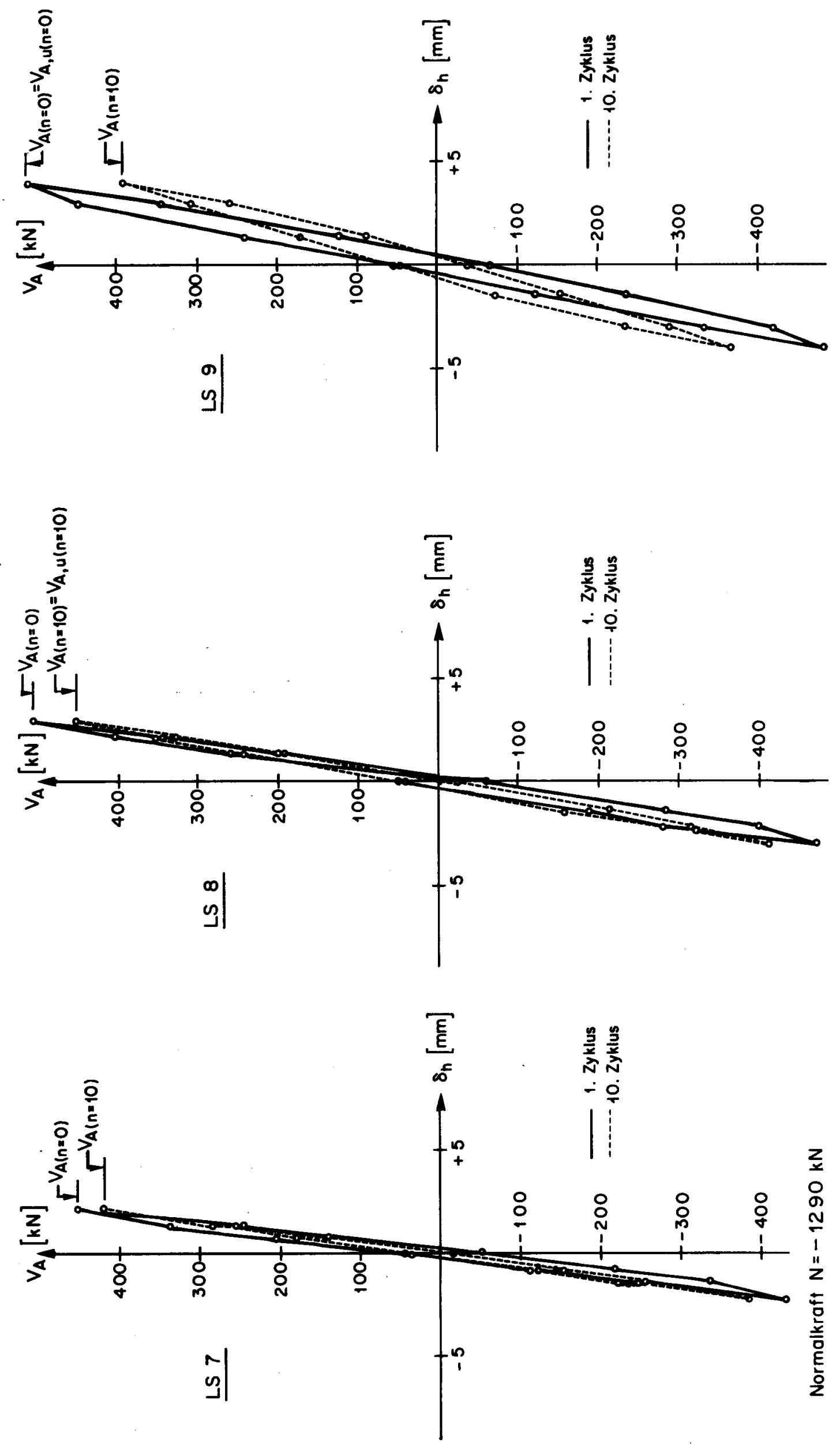

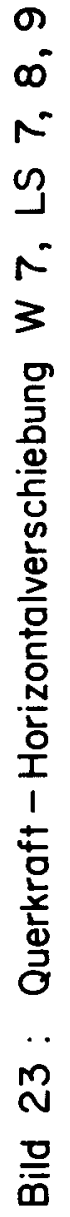



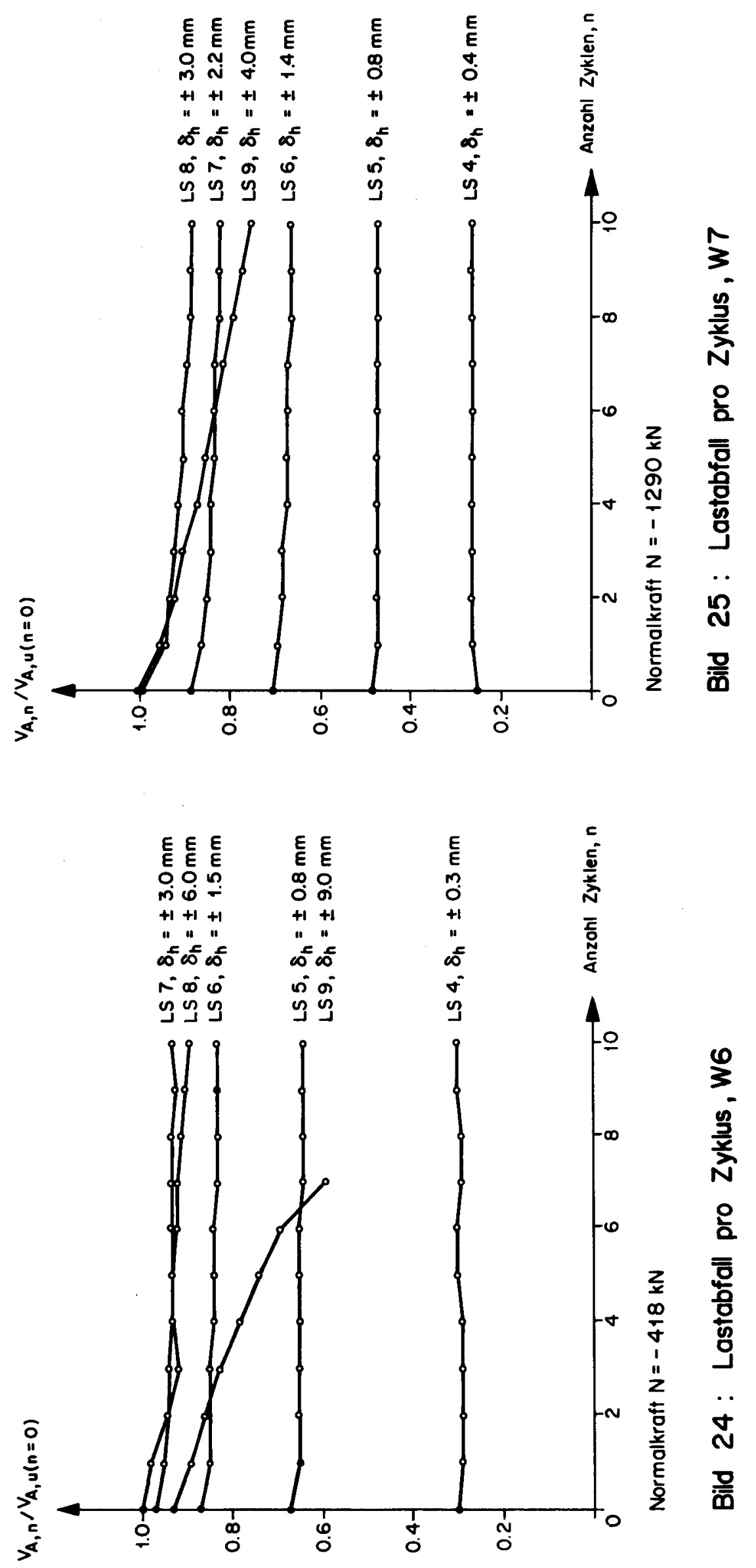


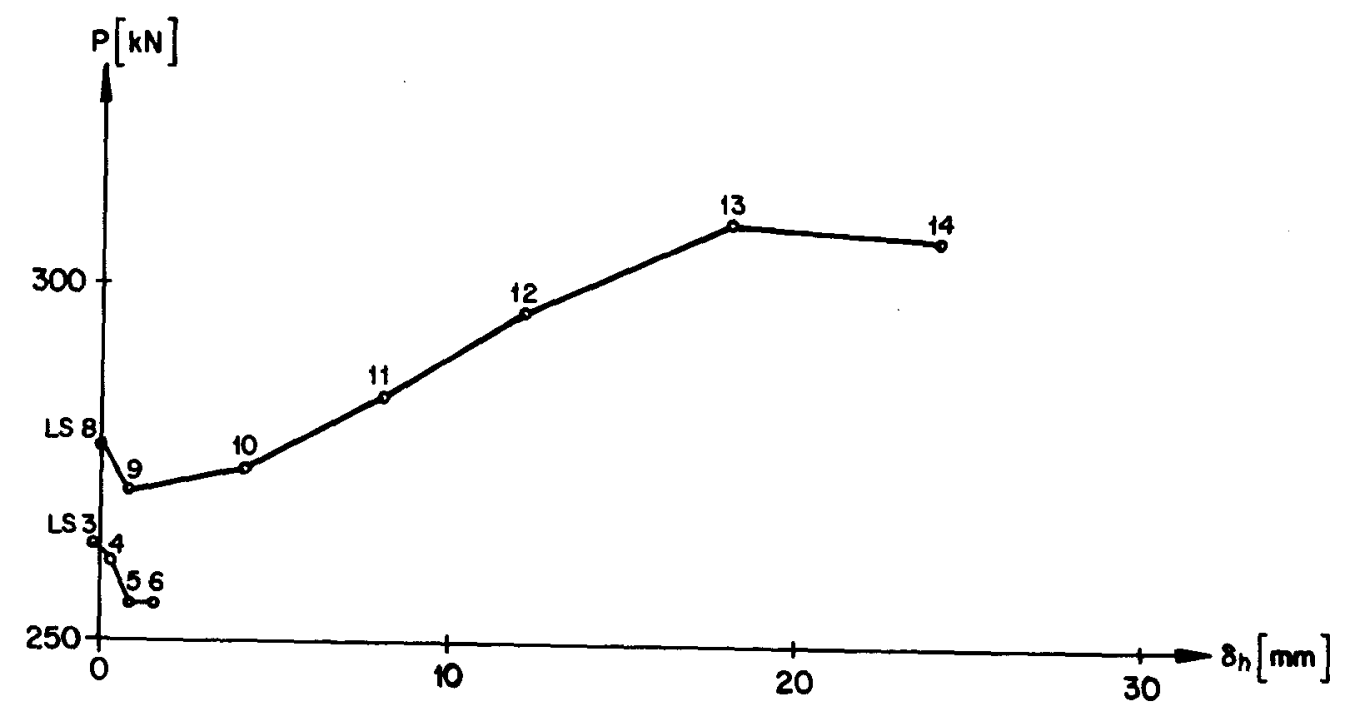

Bild 26 : Verlauf der Vorspannkraft, W5 

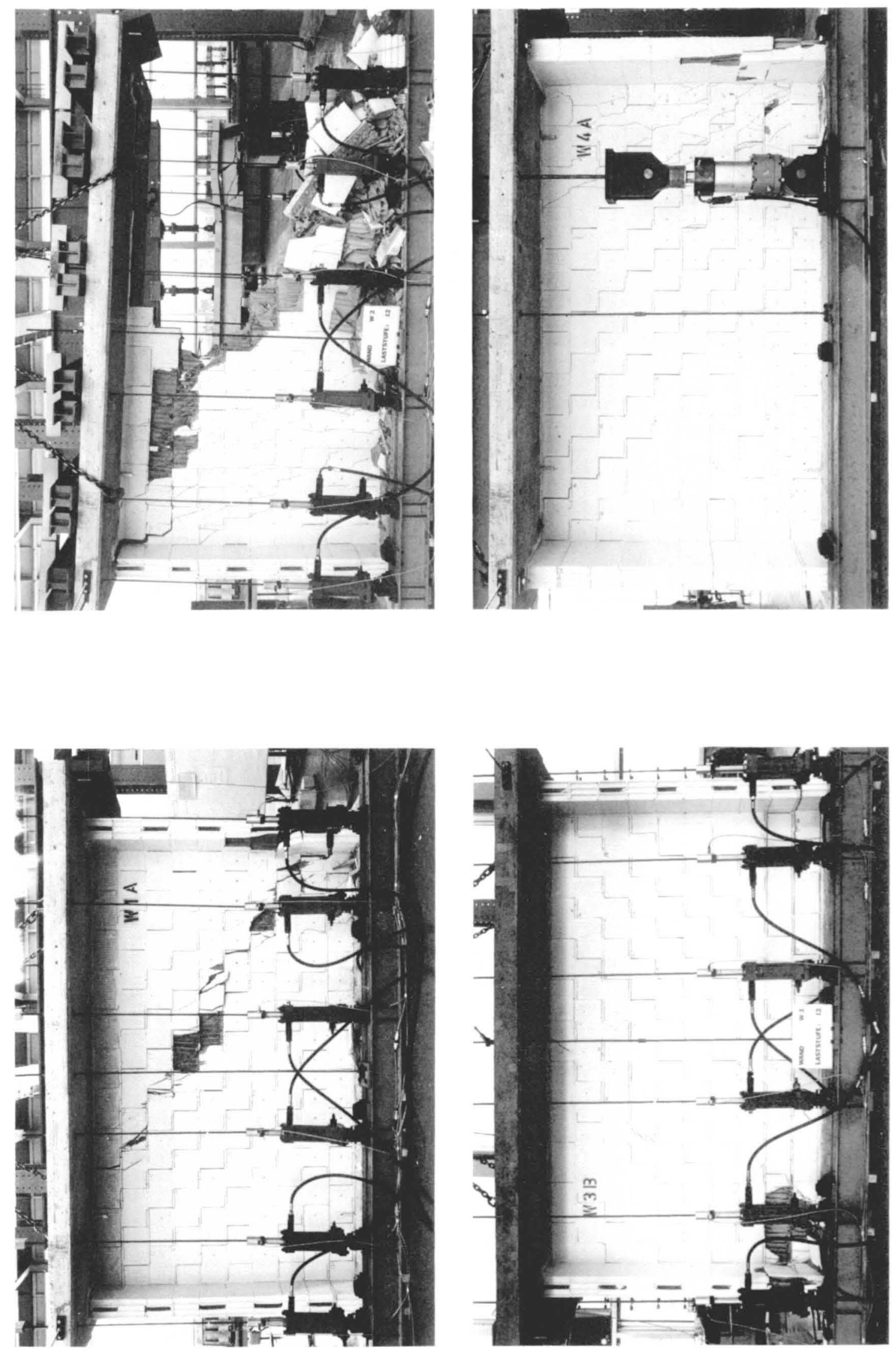

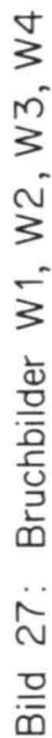



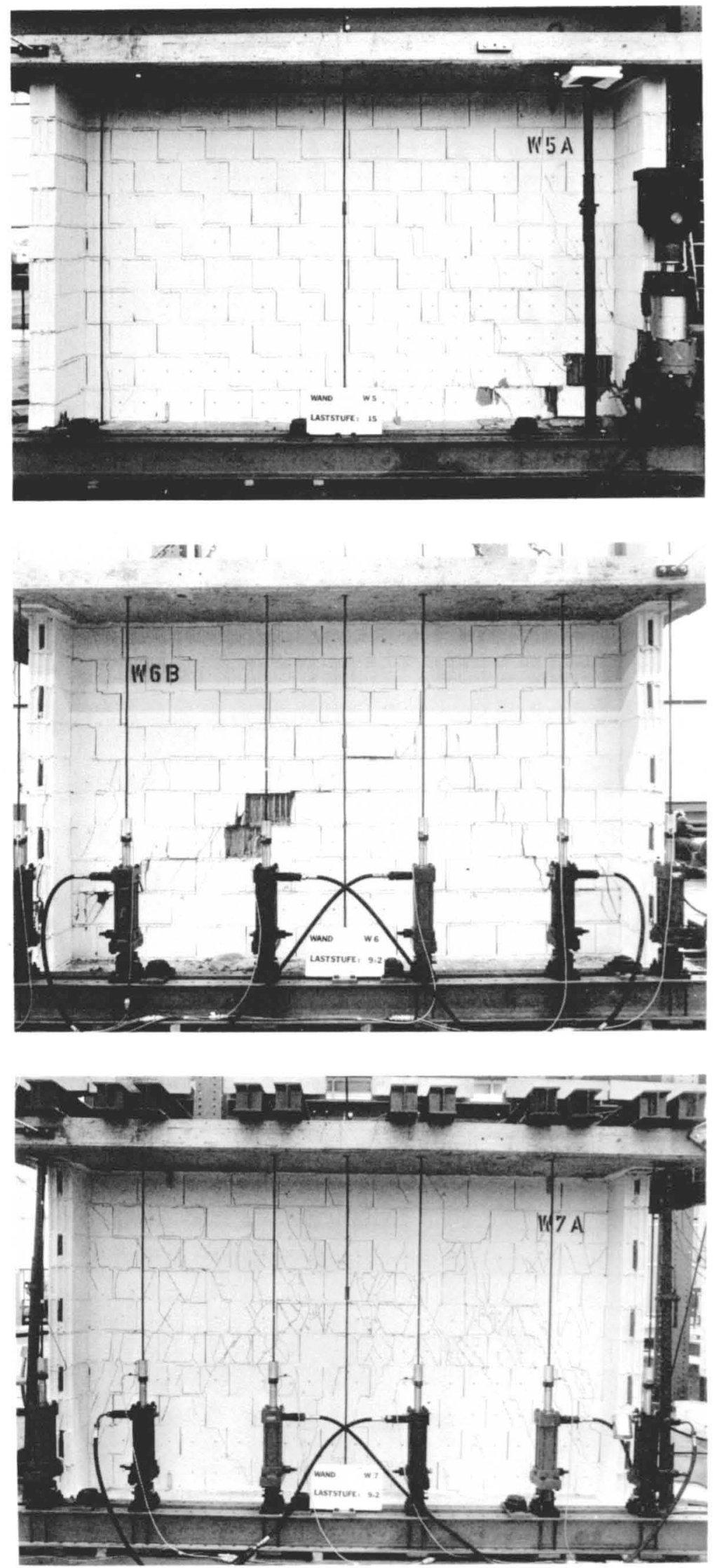

Bild 28: Bruchbilder W5, W6, W7 
Flansch L

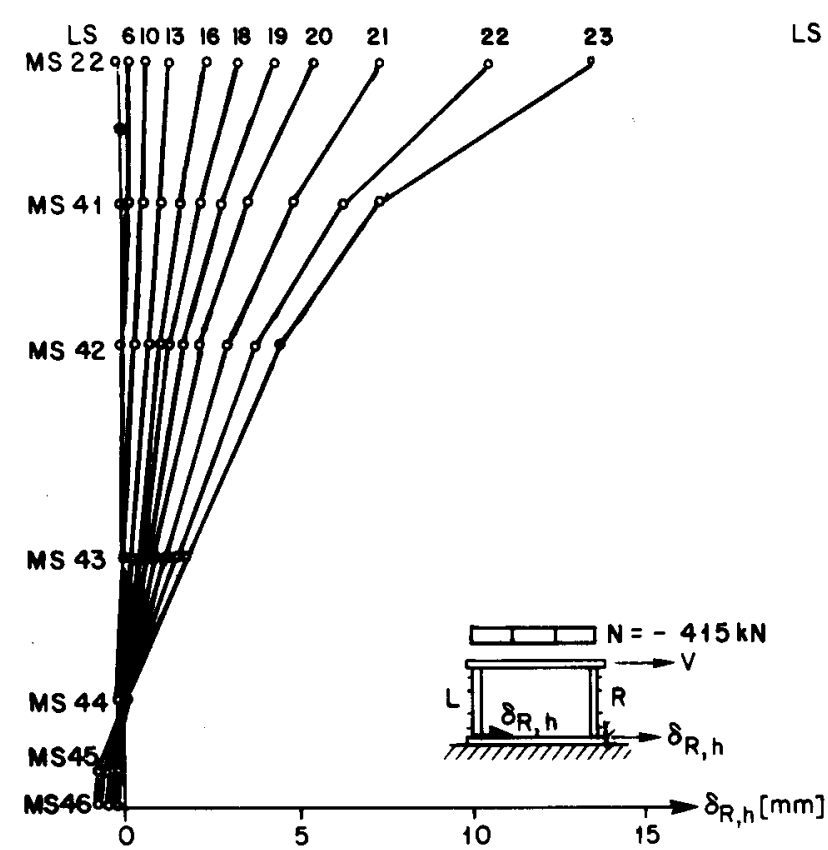

Bild 29: Horizontale Randverschiebungen W 1
Flansch $R$

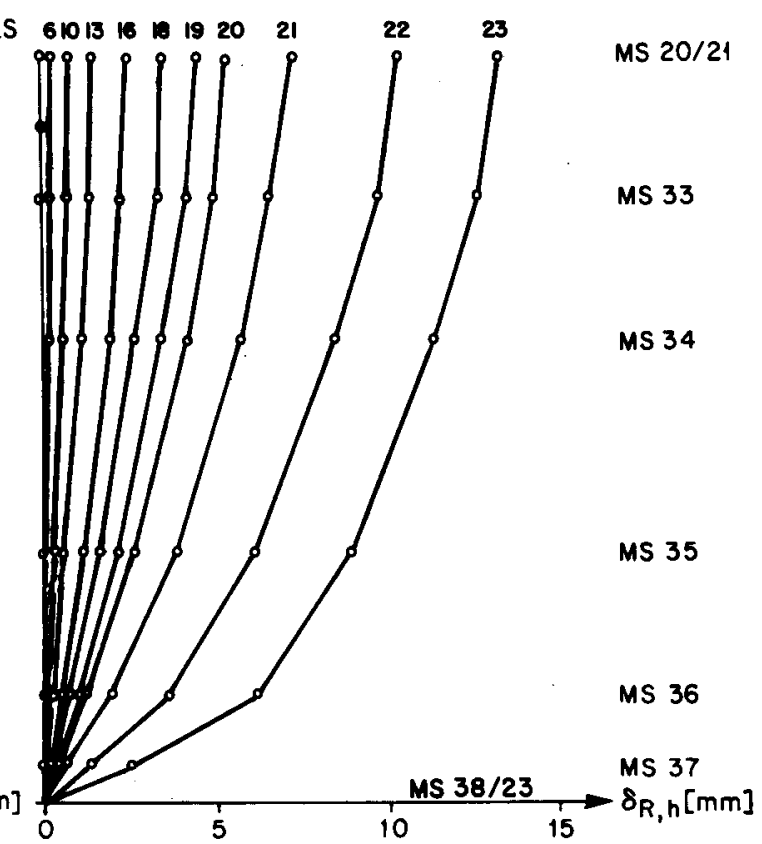

Flansch L

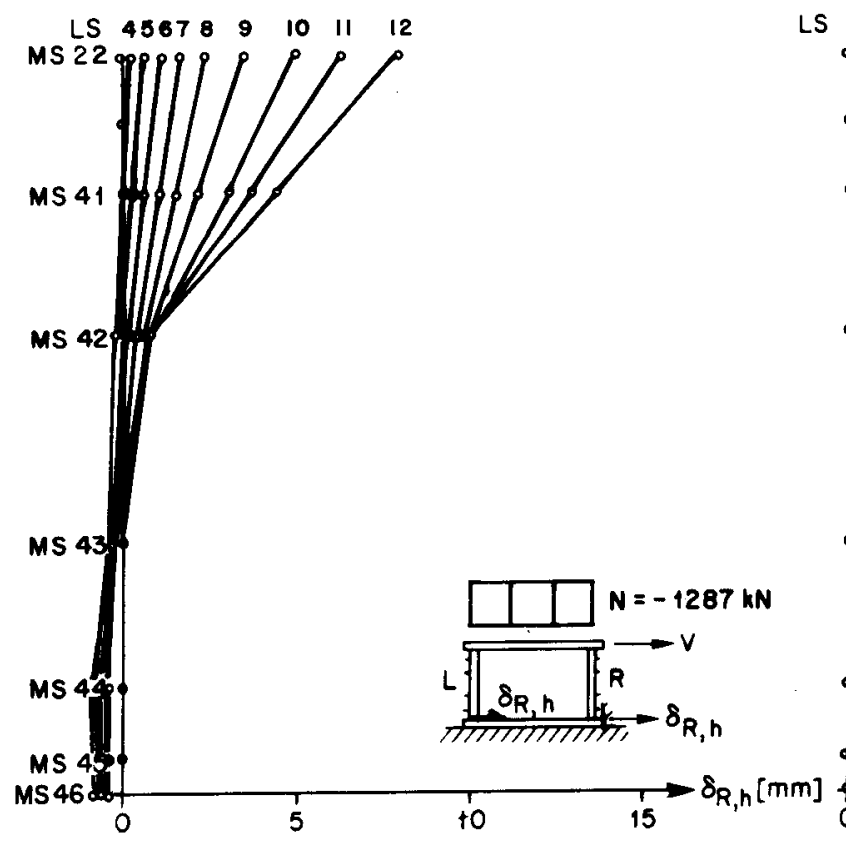

Flonsch R

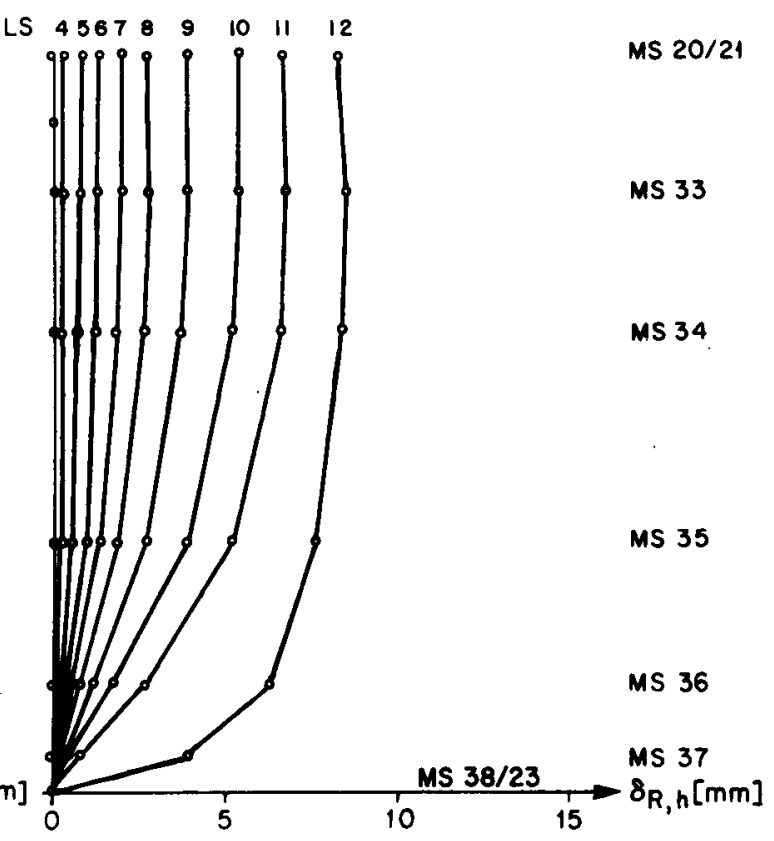

Bild 30: Horizontale Randverschiebungen W 2 
Flansch L

Flansch R

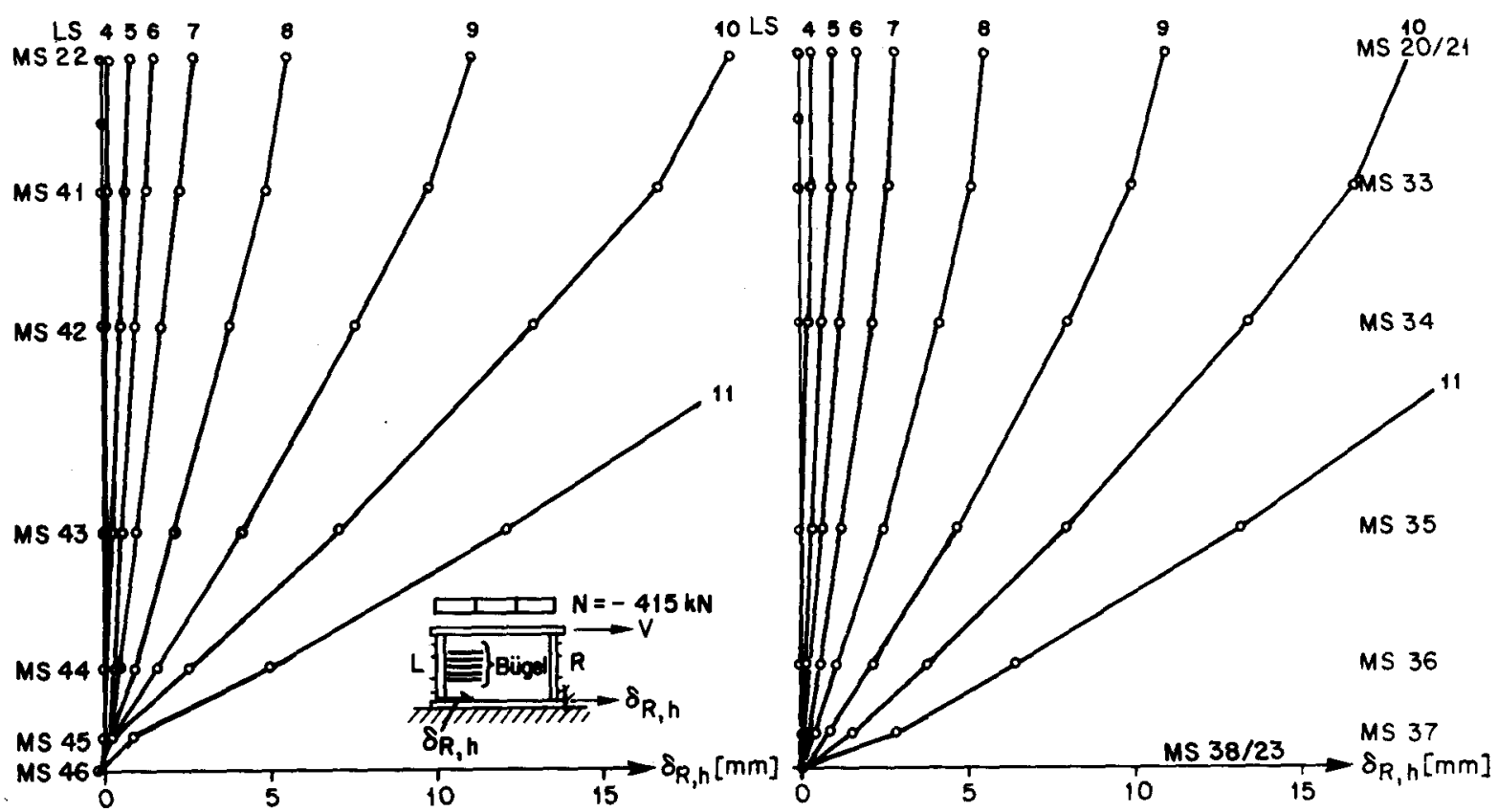

Bild 31: Horizontale Randverschiebungen W 3

Flansch L

Flansch R
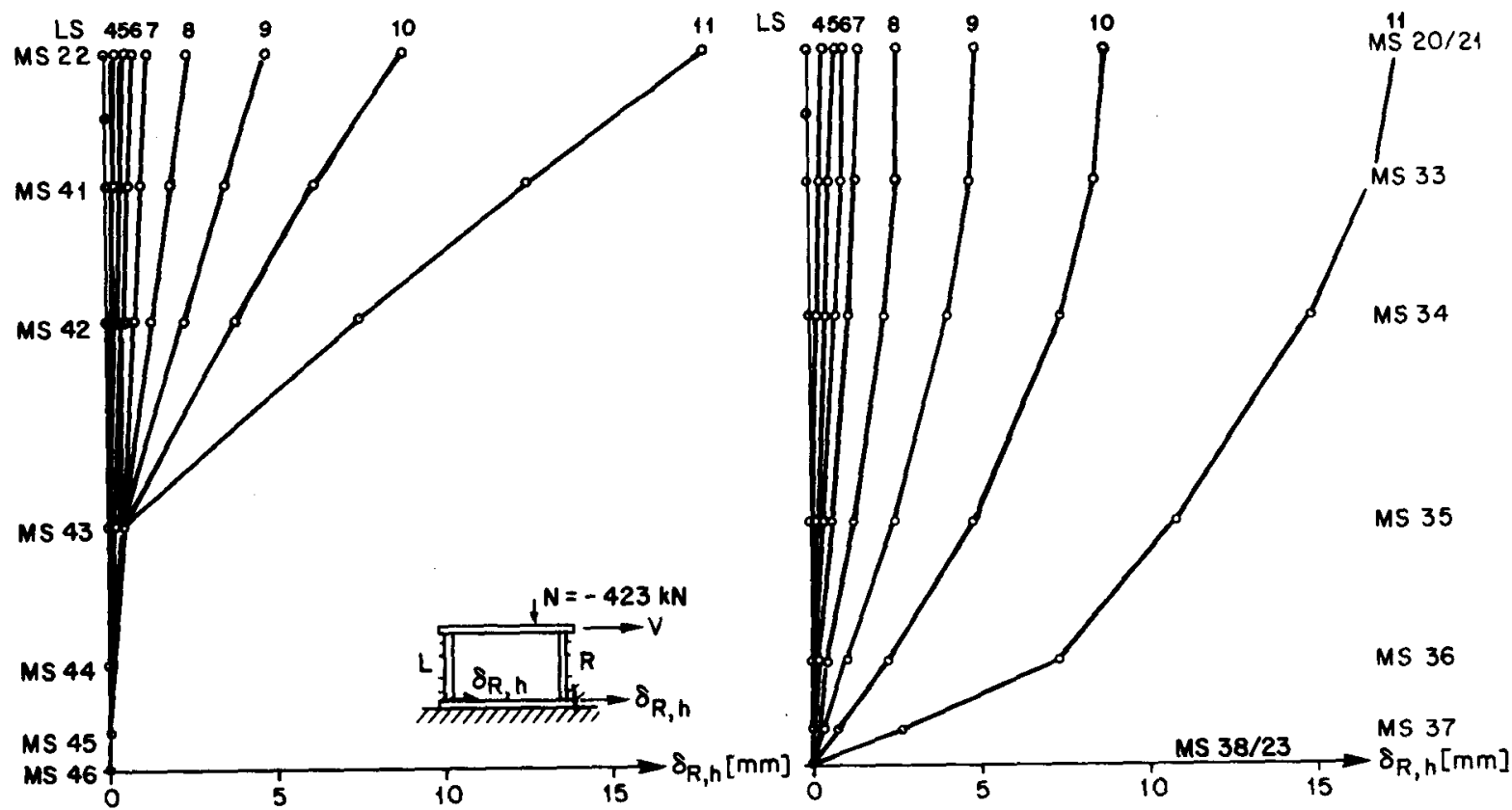

Bild 32: Horizontale Randverschiebungen W 4 
Flansch L

Flonsch R

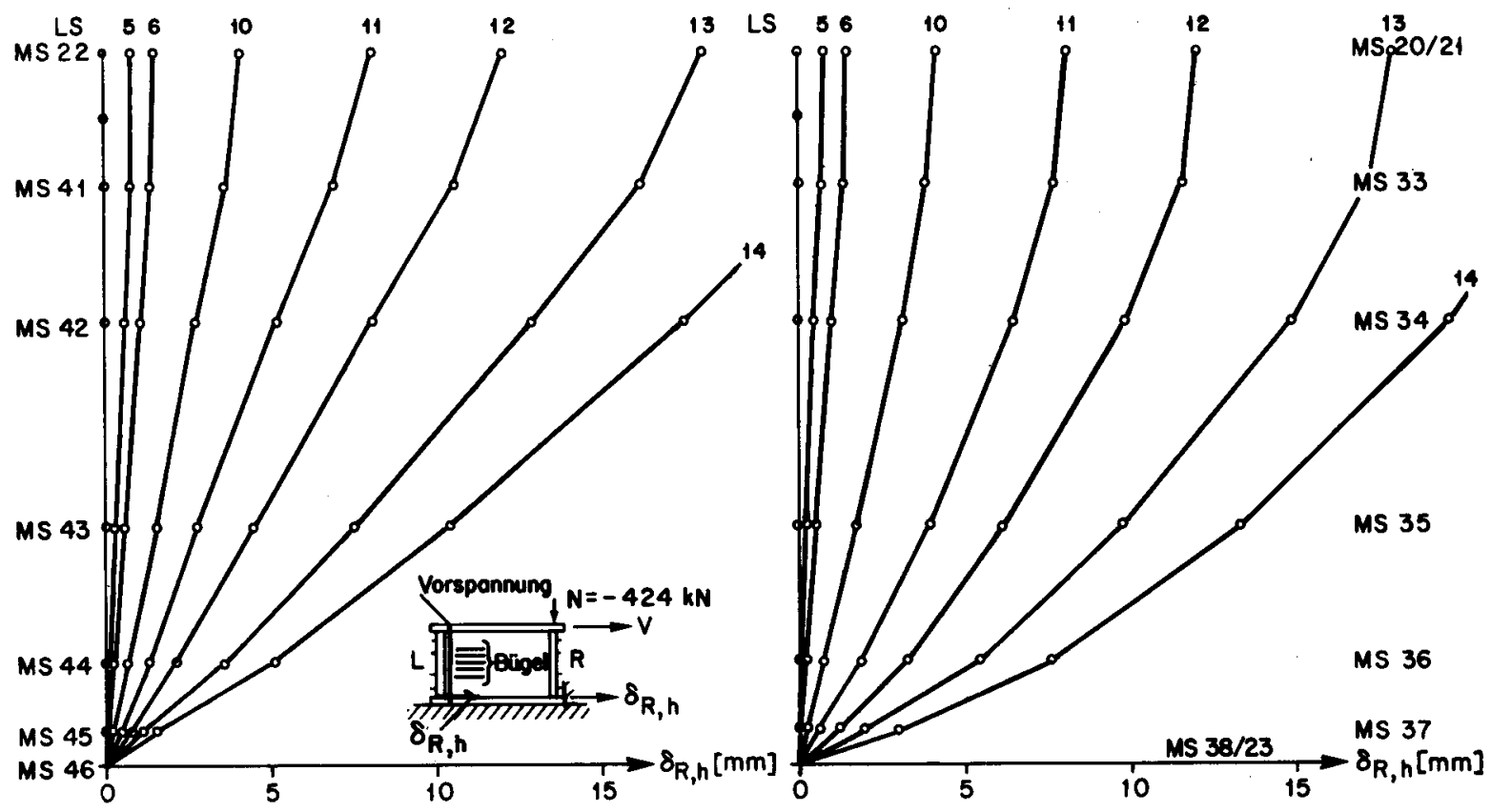

Bild 33: Horizontale Randverschiebungen W 5

Flansch L

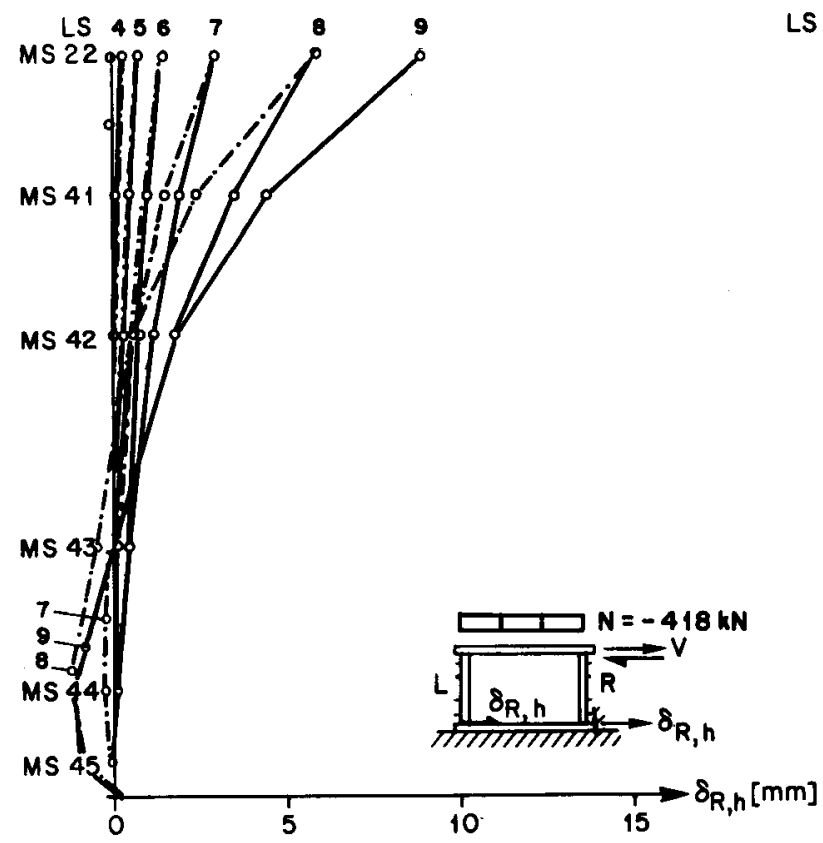

Flansch R

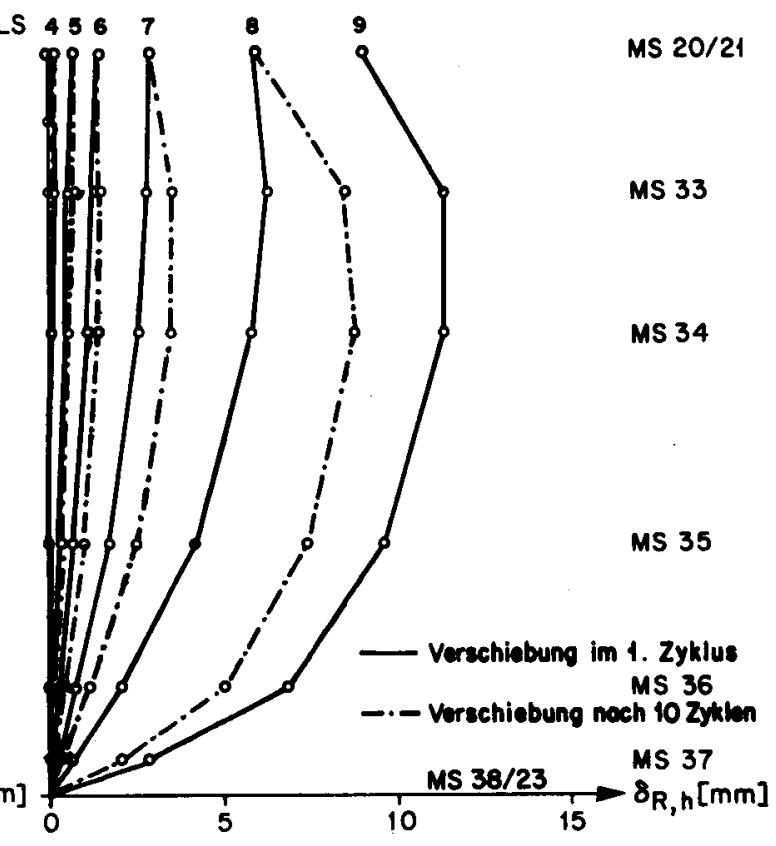

Bild 34: Horizontale Randverschiebungen W 6 
Flansch L

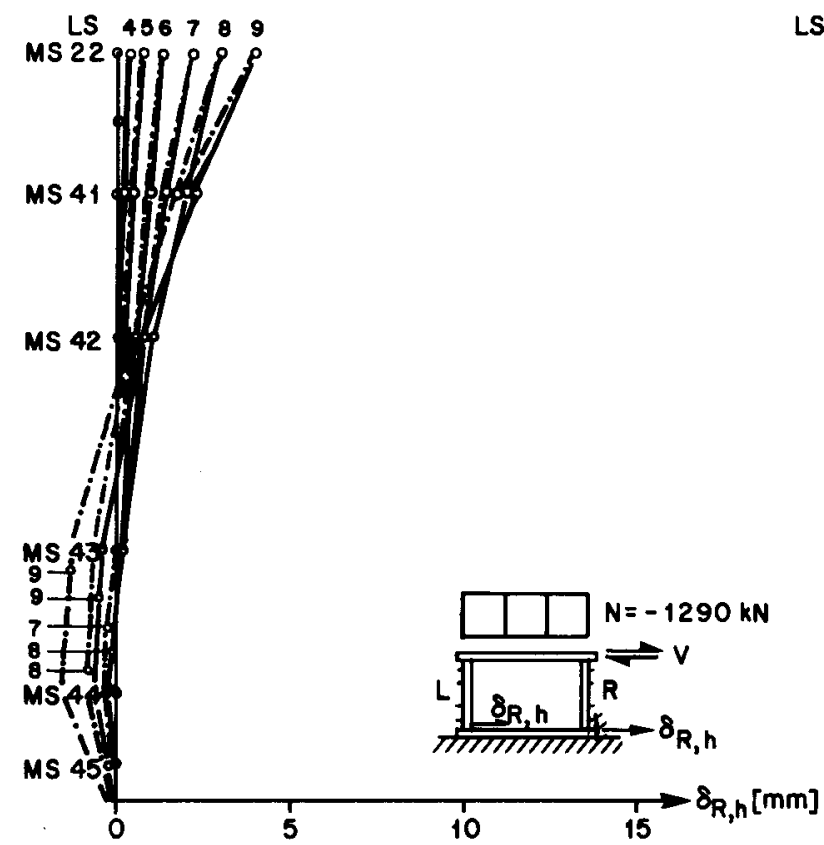

Flansch $R$

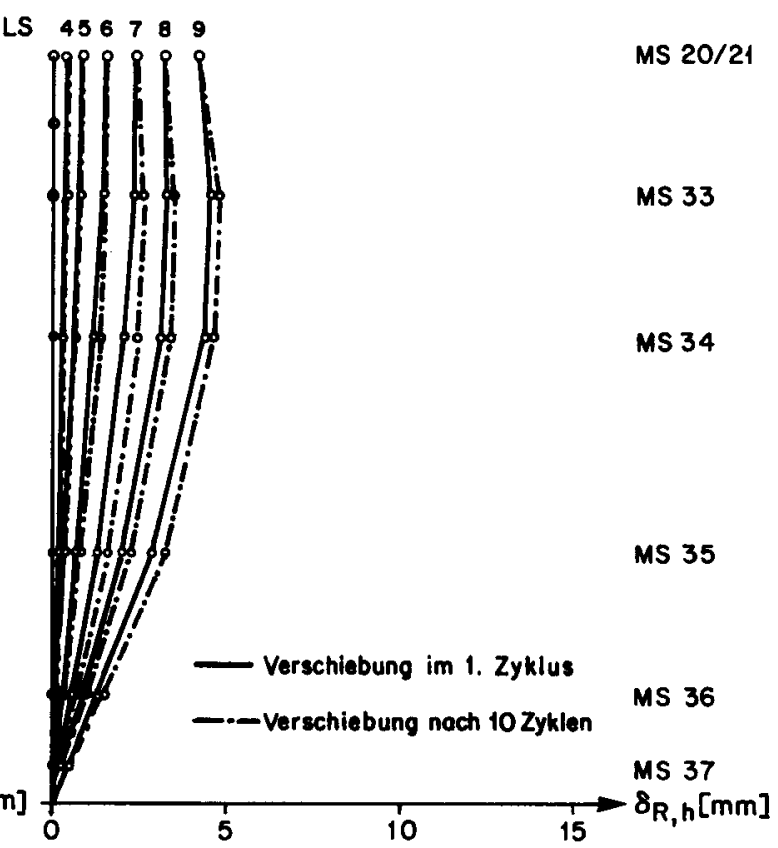

Bild 35: Horizontale Randverschiebungen $W 7$

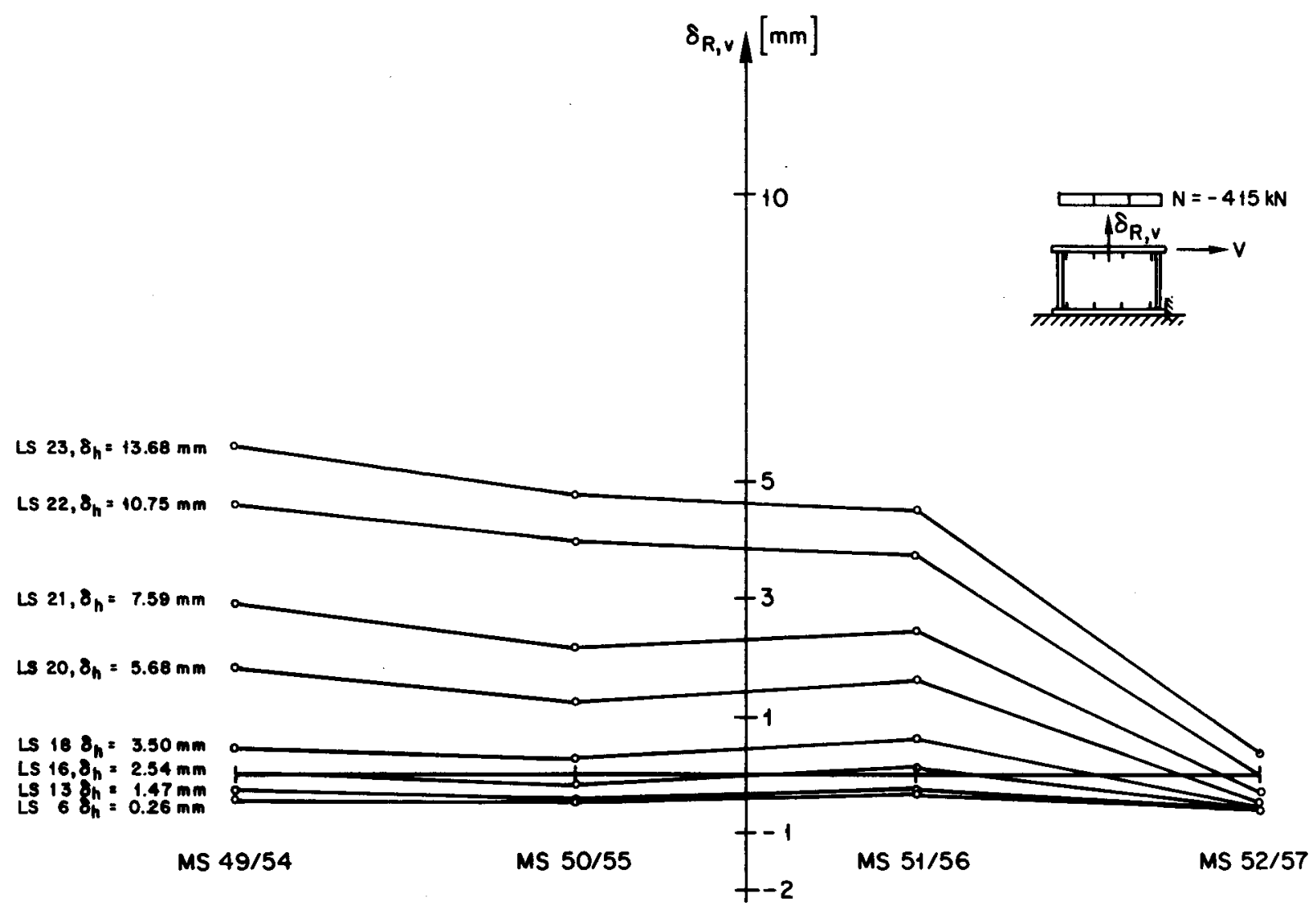

Bild 36: Vertikale Randverschiebungen W 1 


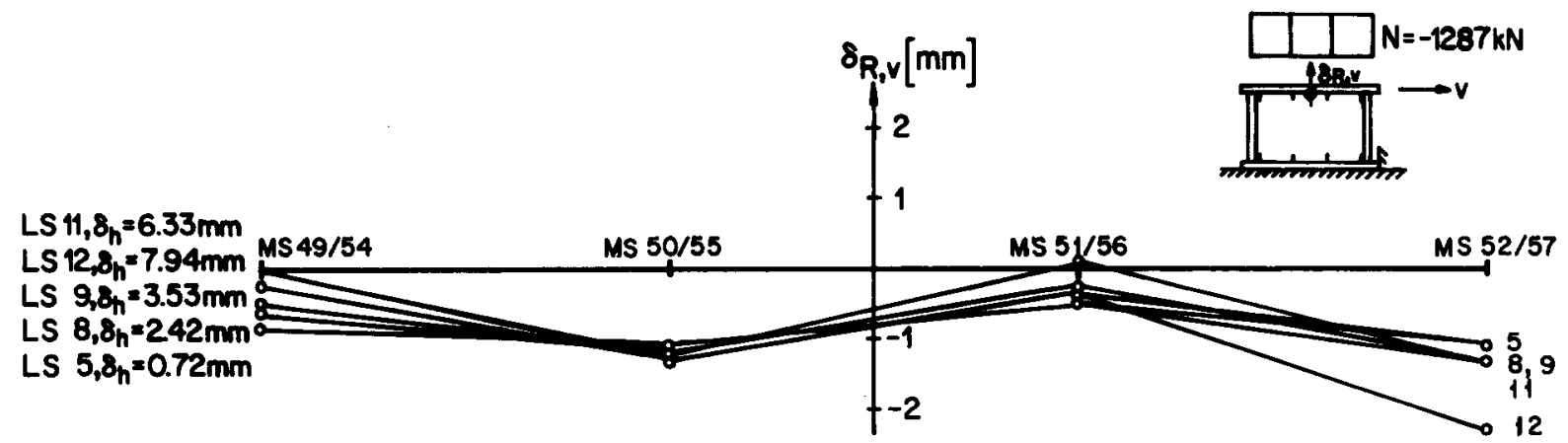

Bild 37 : Vertikale Randverschiebungen W2

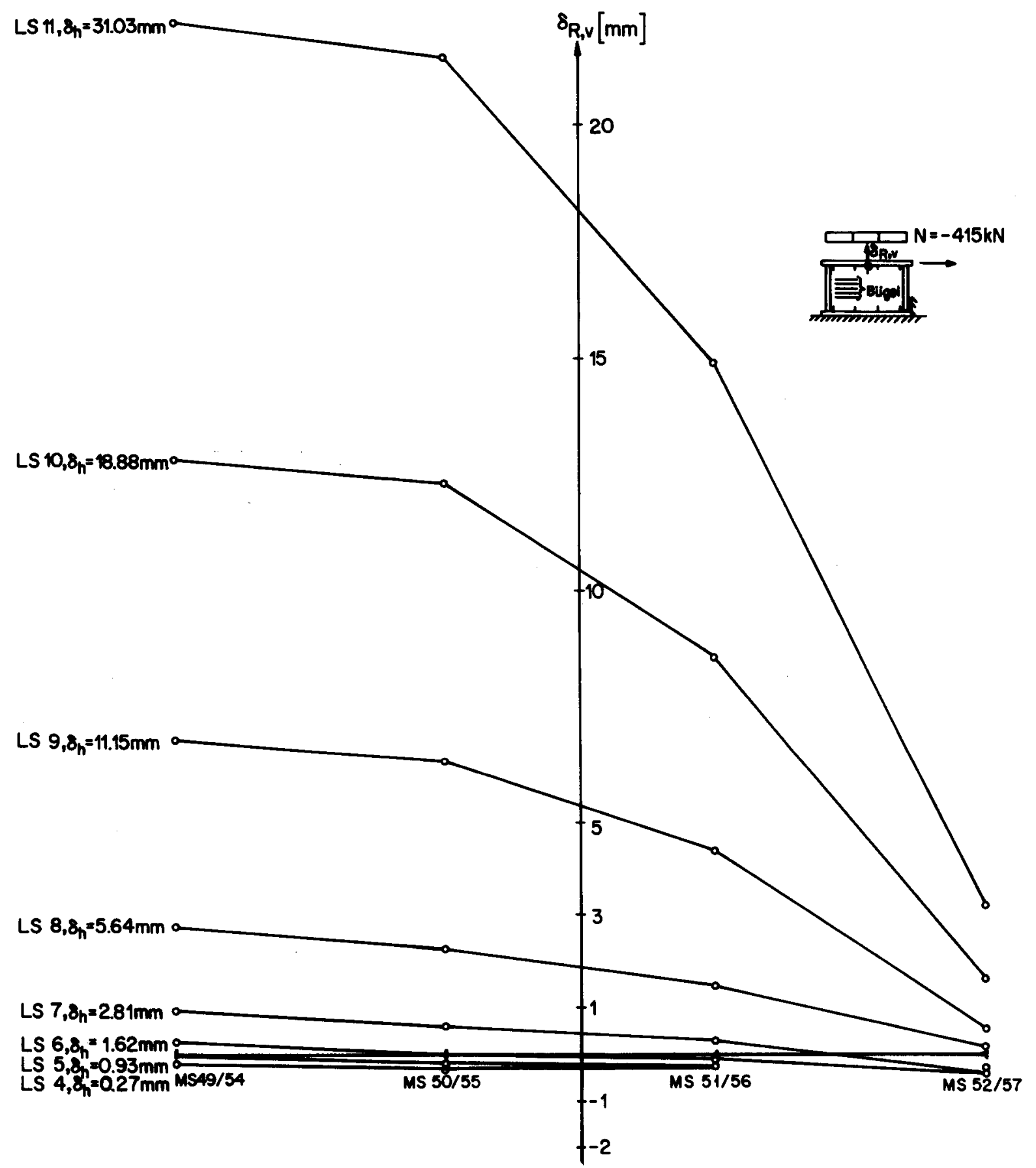

Bild 38 : Vertikde Randverschiebungen W 3 


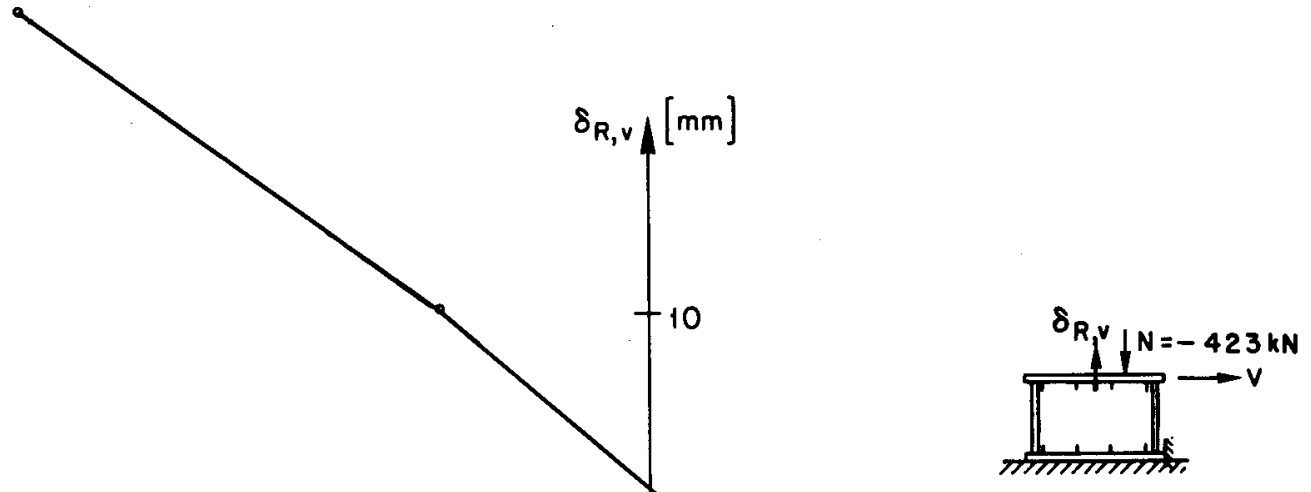

LS 10, $8_{h}=8.84 \mathrm{~mm}$

LS $9,8_{h}=4.82 \mathrm{~mm}$

LS $8,8_{h}=2.45 \mathrm{~mm}$

LS $7,8_{h}=1.26 \mathrm{~mm}$

LS $5,8_{h}=0.58 \mathrm{~mm}$

LS $4,8_{h}=0.29 \mathrm{~mm}$

MS 49/54

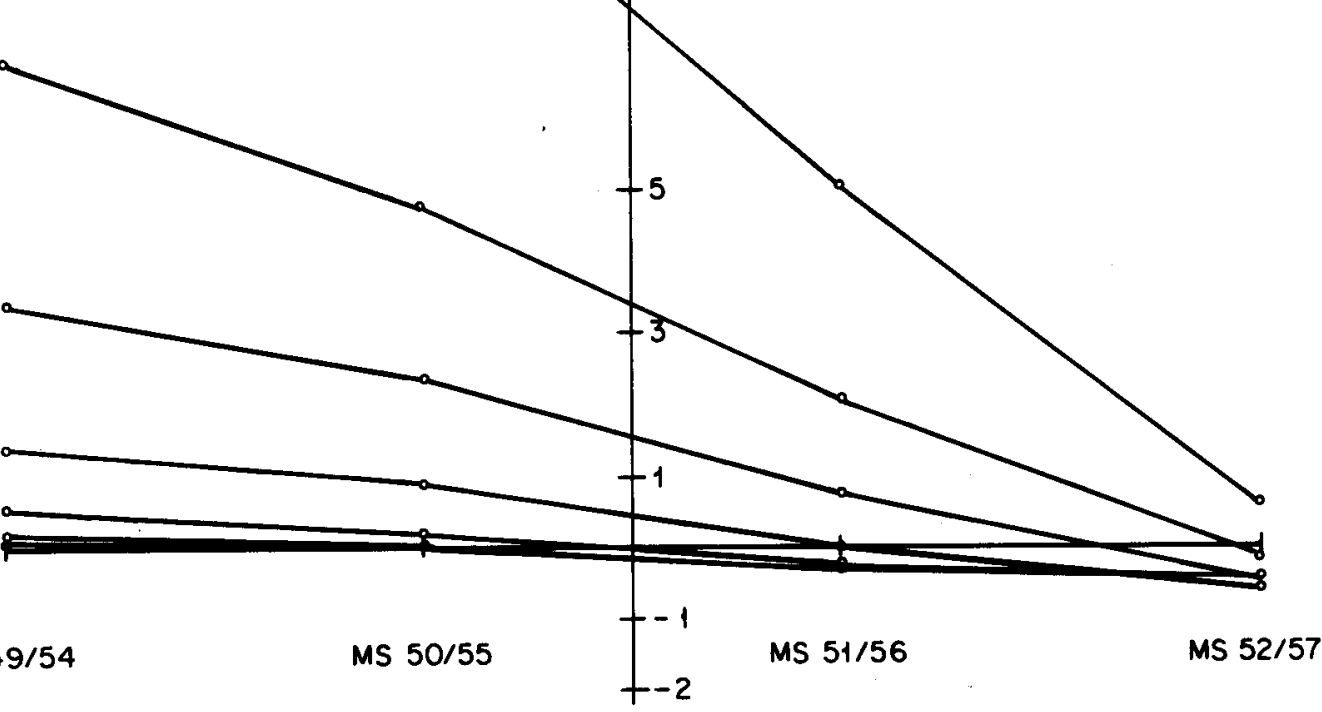

Bild 39: Vertikale Randverschiebungen W 4

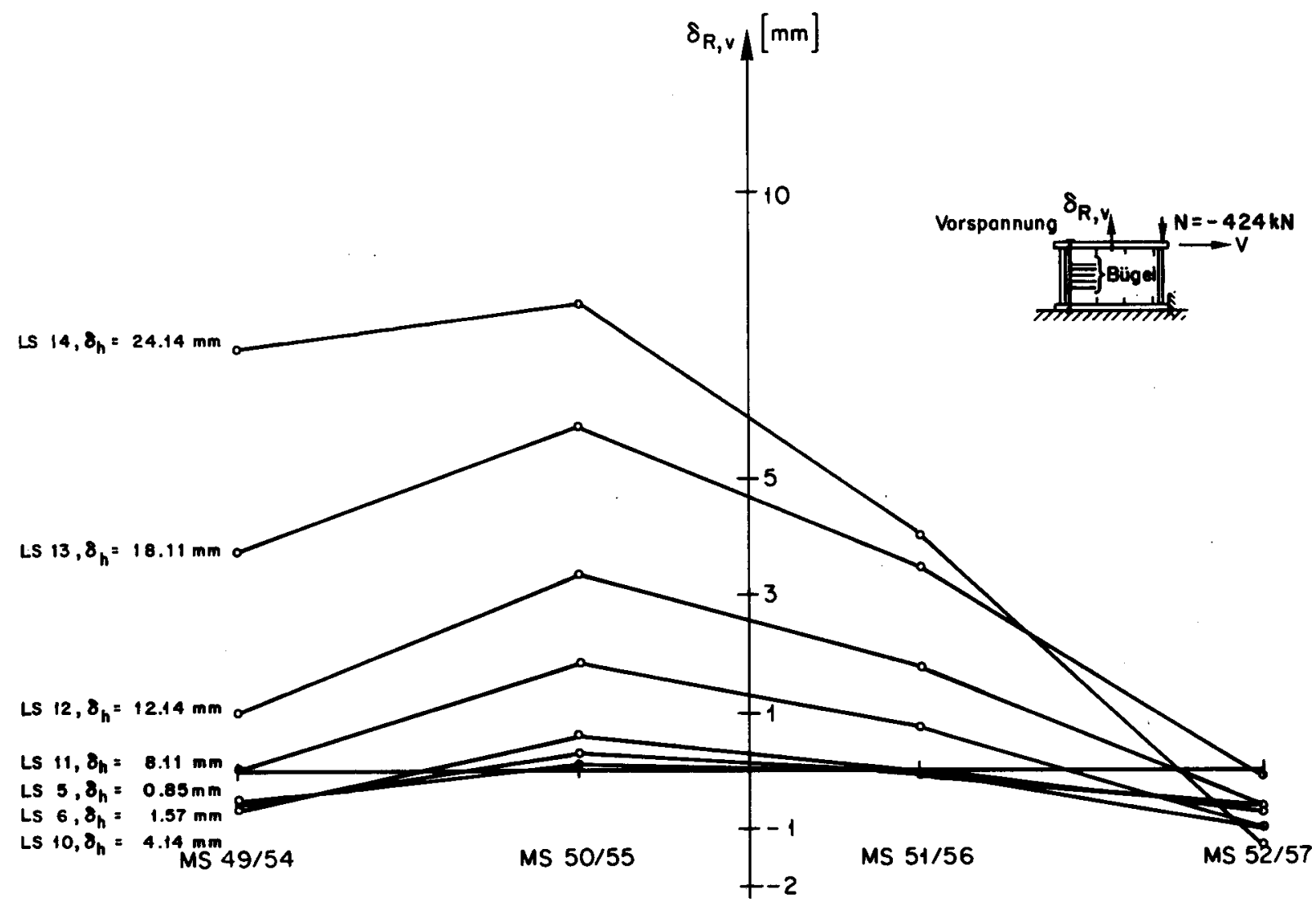

Bild 40: Vertikale Randverschiebungen W 5 


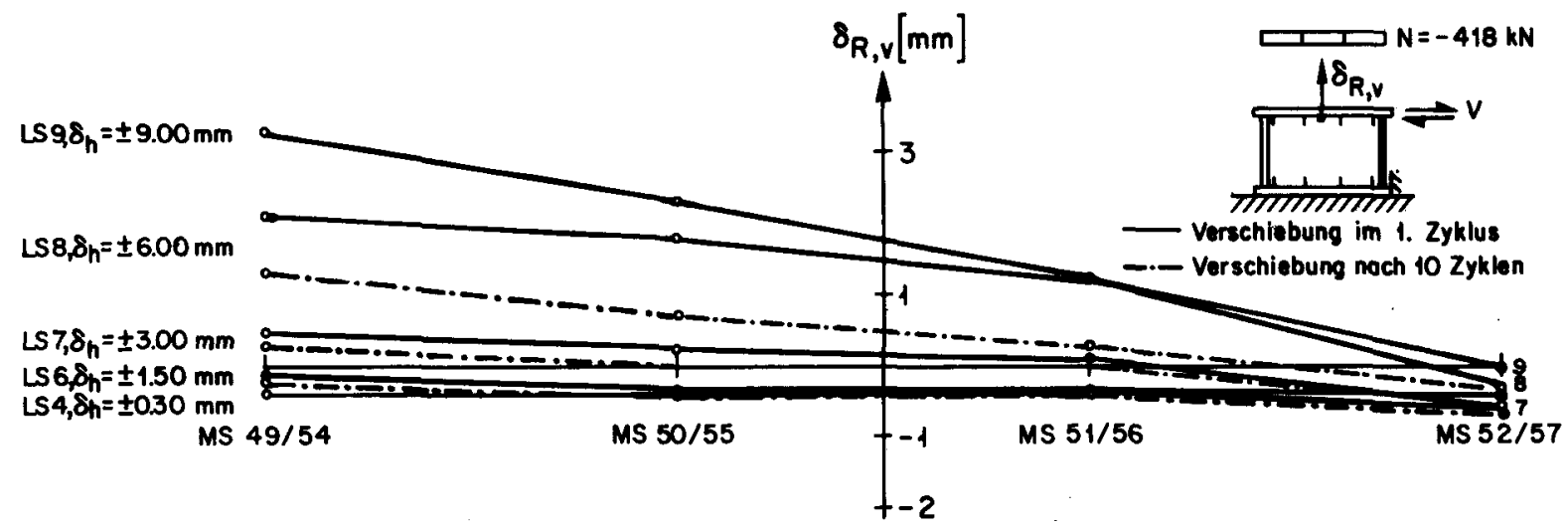

Bild 41 : Vertikale Randverschiebungen W6

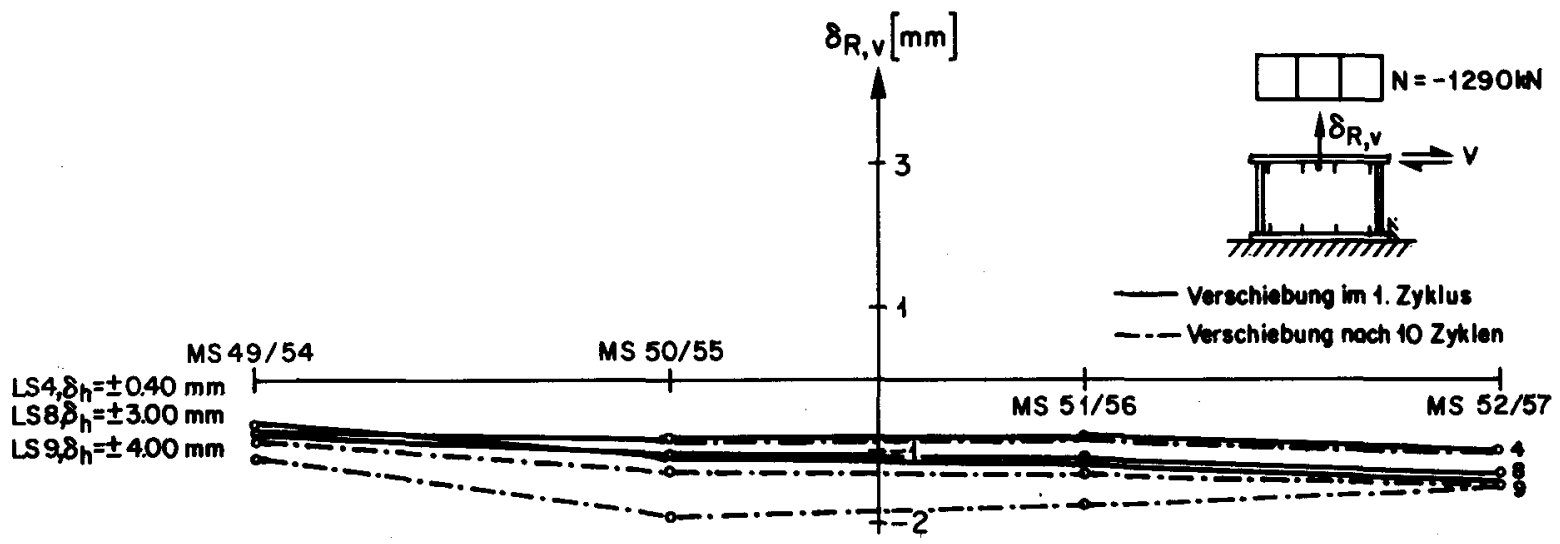

Bild 42 : Vertikale Randverschiebungen W7

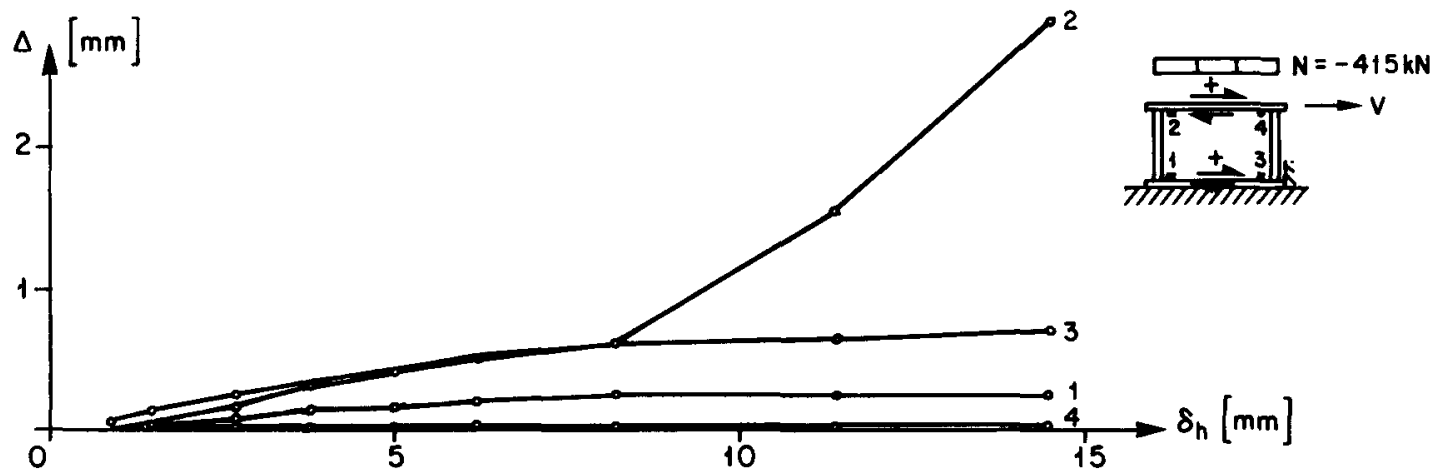

Bild 43 : Relativverschiebung Wand-Betonplatten W 1

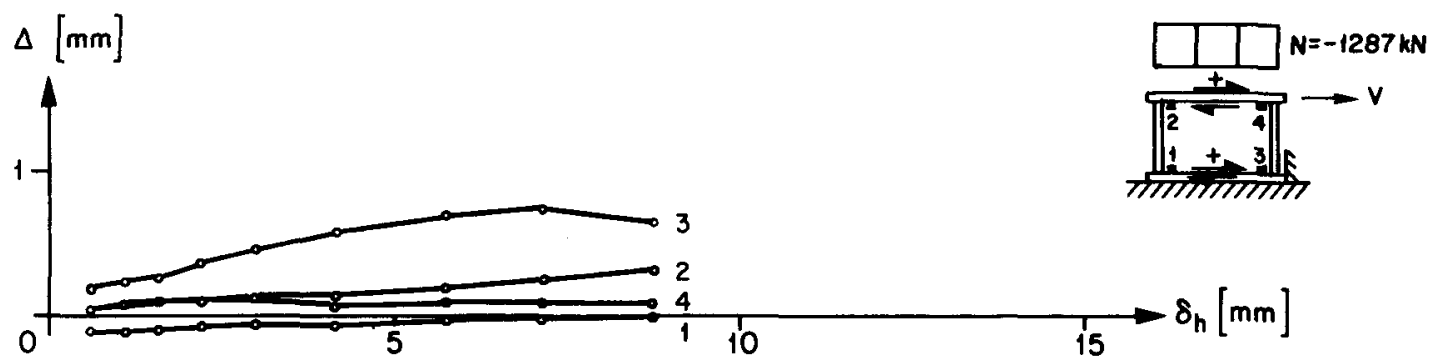

Bild 44 : Relativverschiebung Wand-Betonplatten W 2 


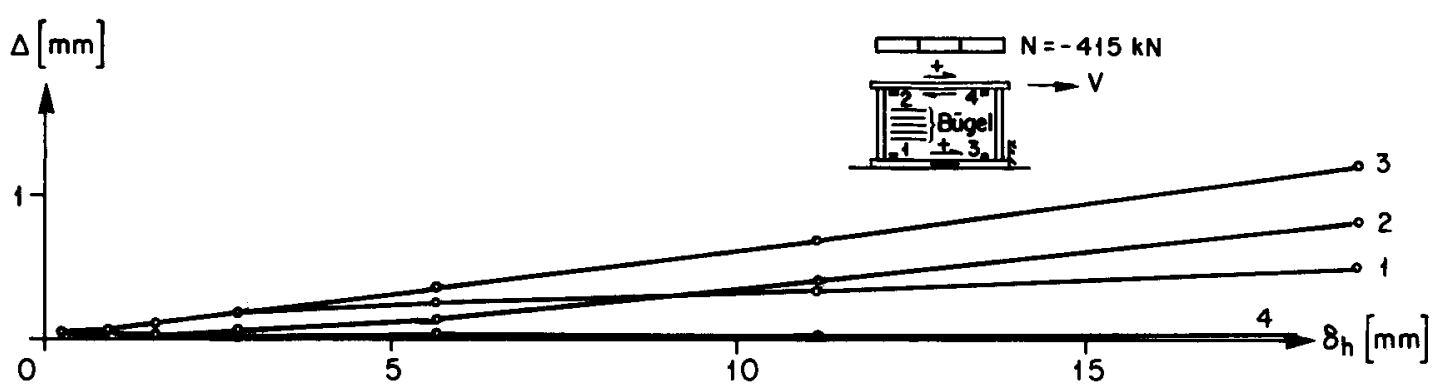

Bild 45 : Relativverschiebung Wand-Betonplatten W 3

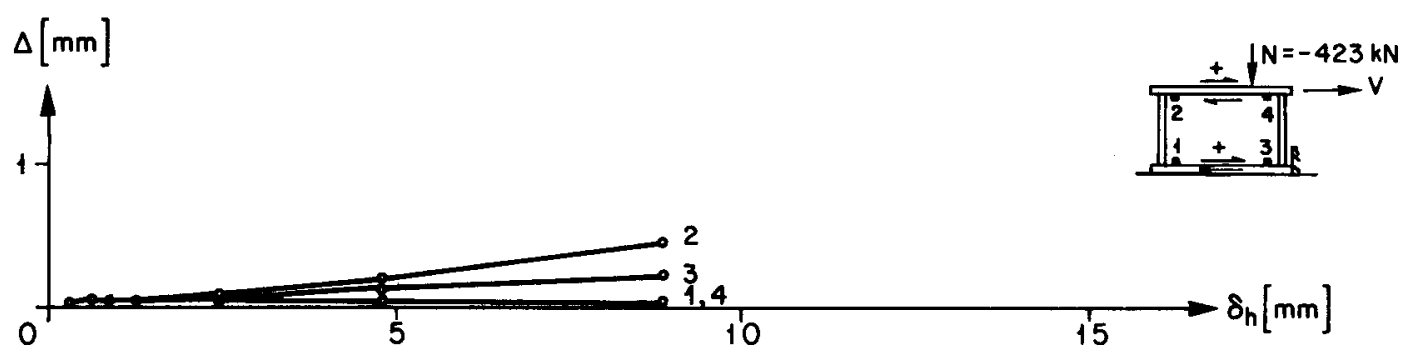

Bild 46 : Relativverschiebung Wand-Betonplatten W 4

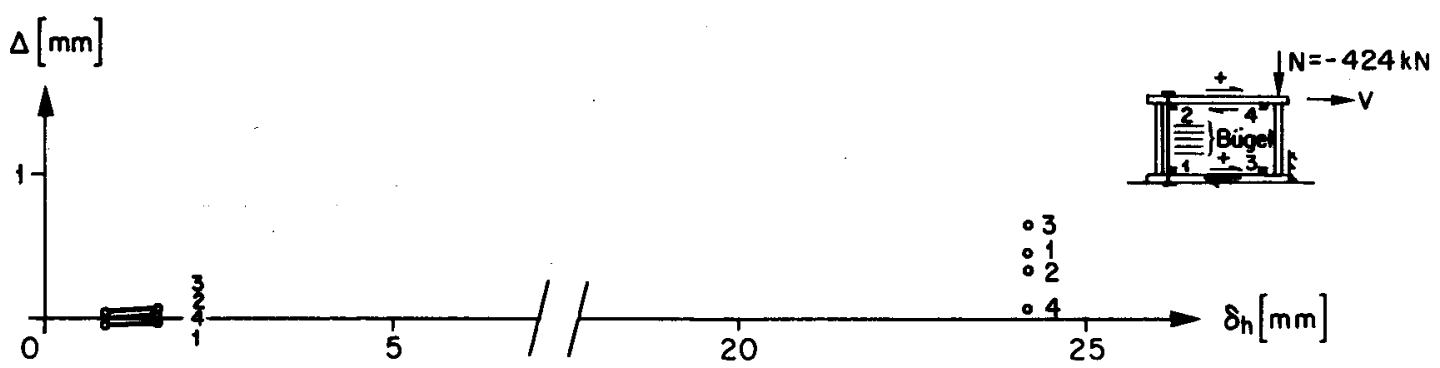

Bild 47 : Relativverschiebung Wand-Betonplatten W 5

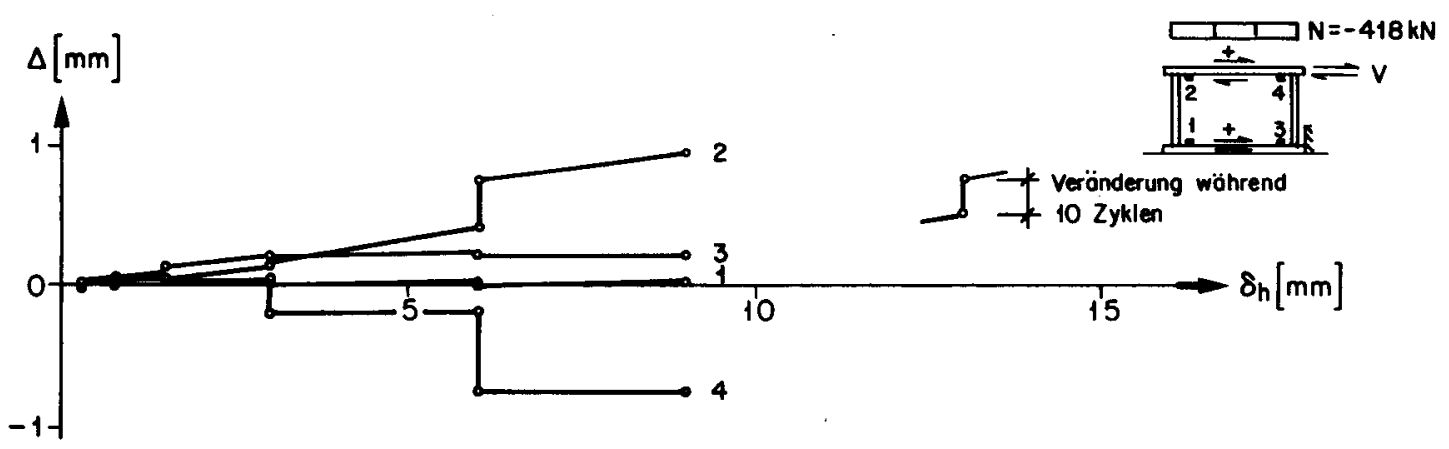

Bild 48 : Relativverschiebung Wand-Betonplatten W 6

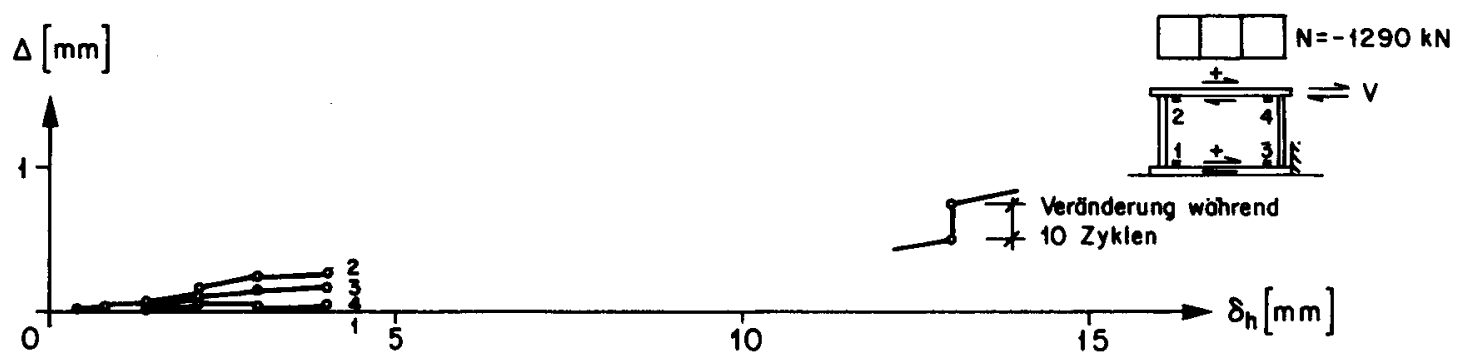

Bild 49 : Relativverschiebung Wand-Bettonplatten W 7 

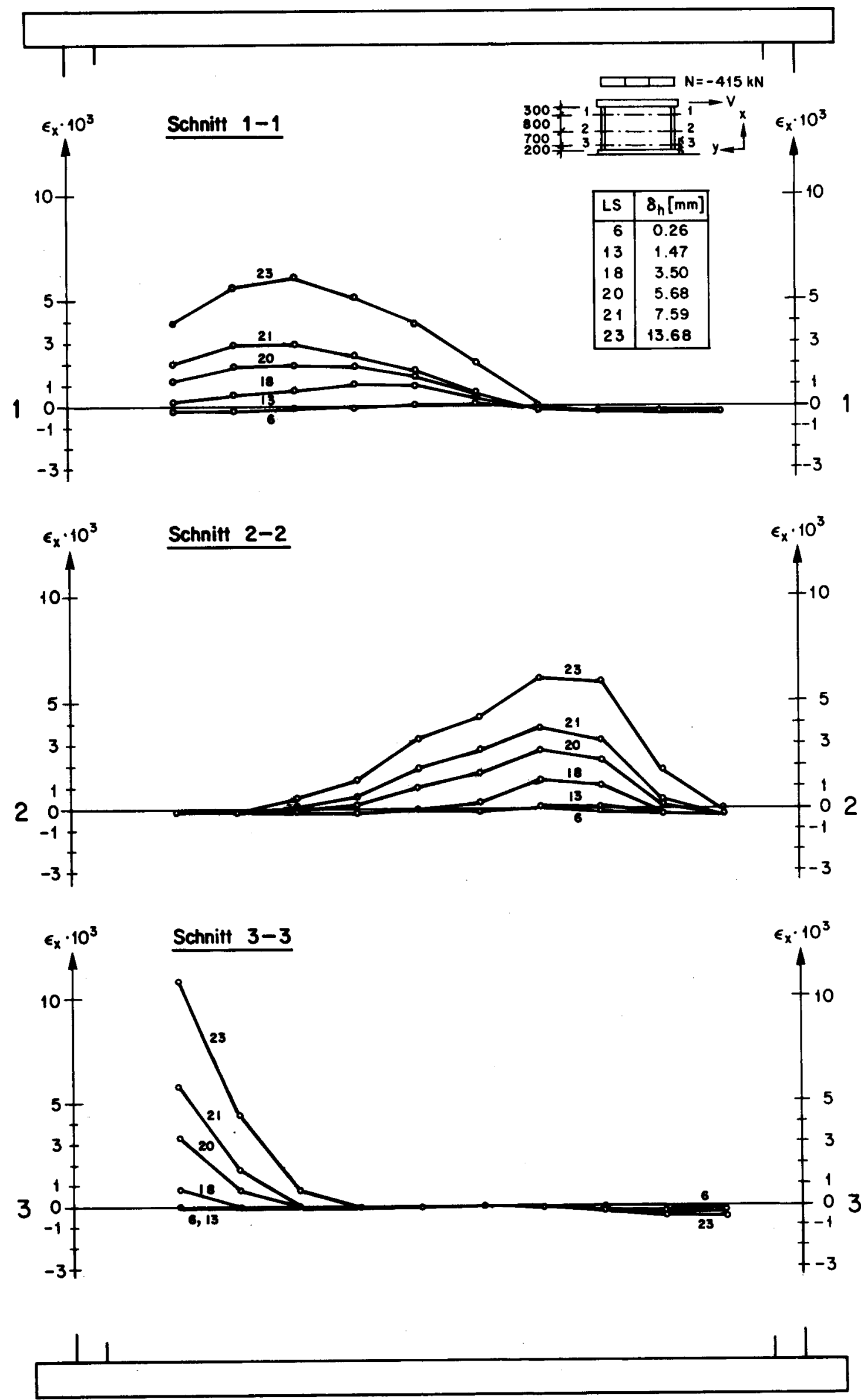

Bild 50: Dehnungen $\epsilon_{\mathrm{x}}, W 1$ 

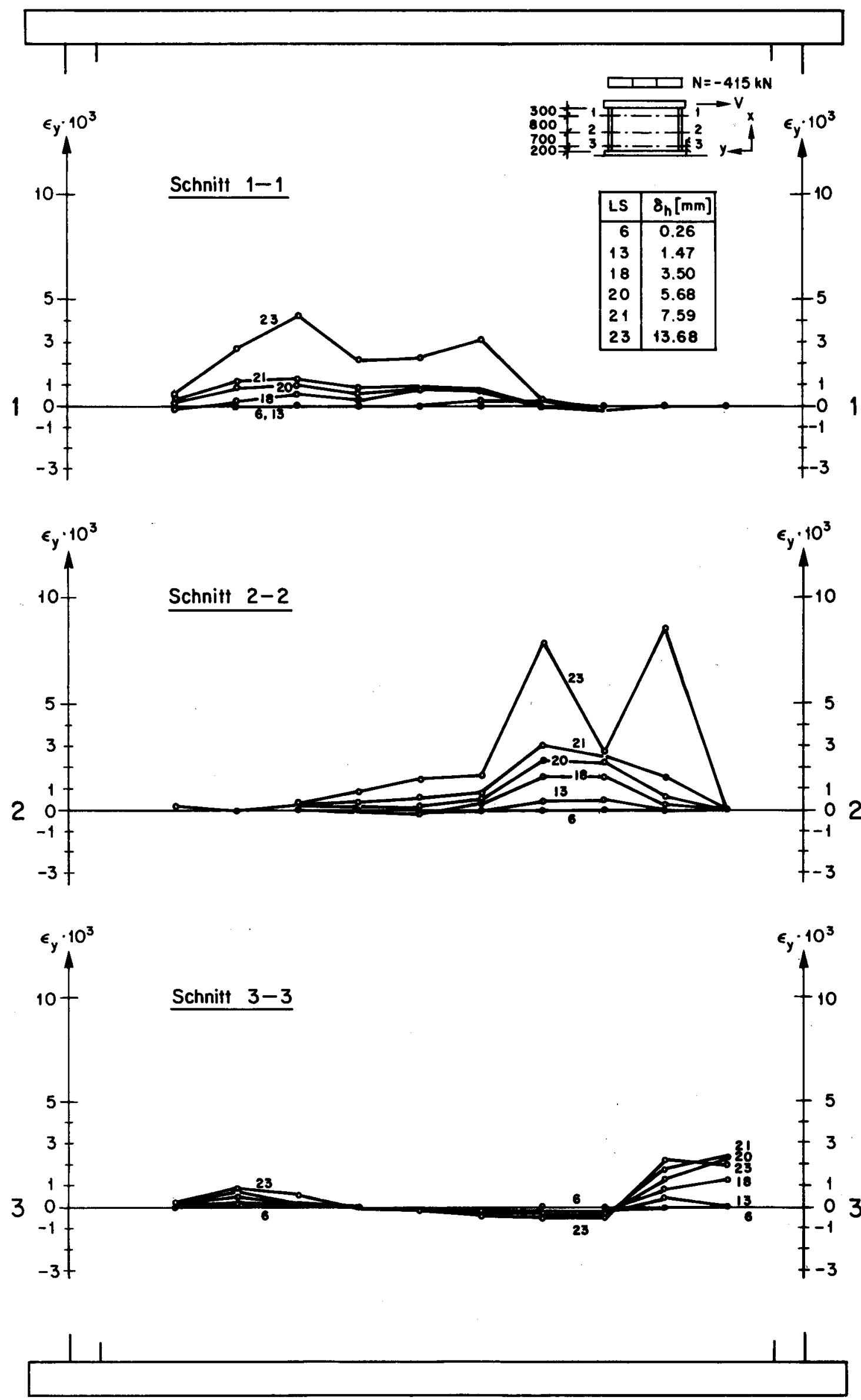

Bild 51: Dehnungen $\epsilon_{\mathrm{y}}, W 1$ 


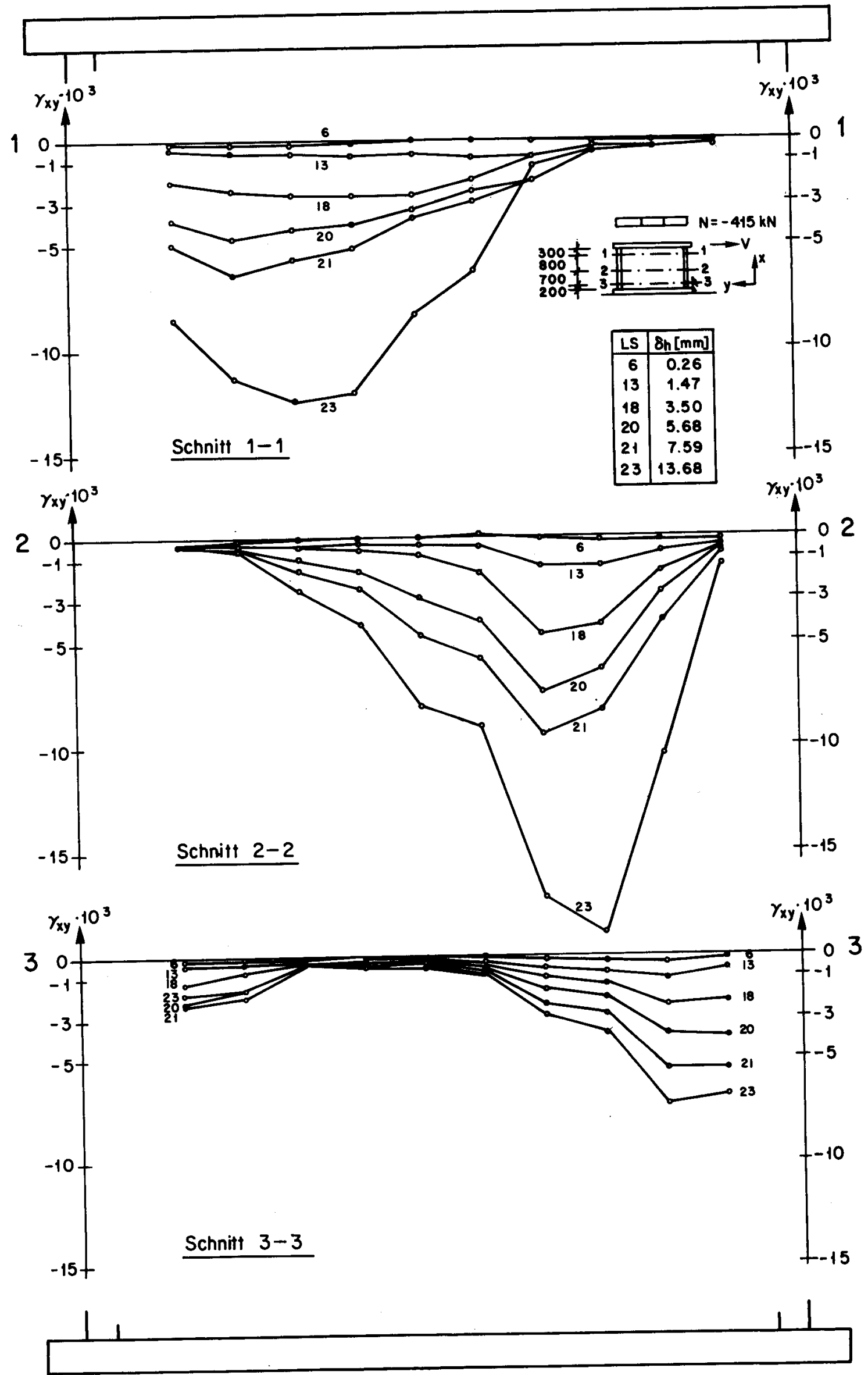

Bild 52: Schiebungen $\gamma_{x y}, w 1$ 

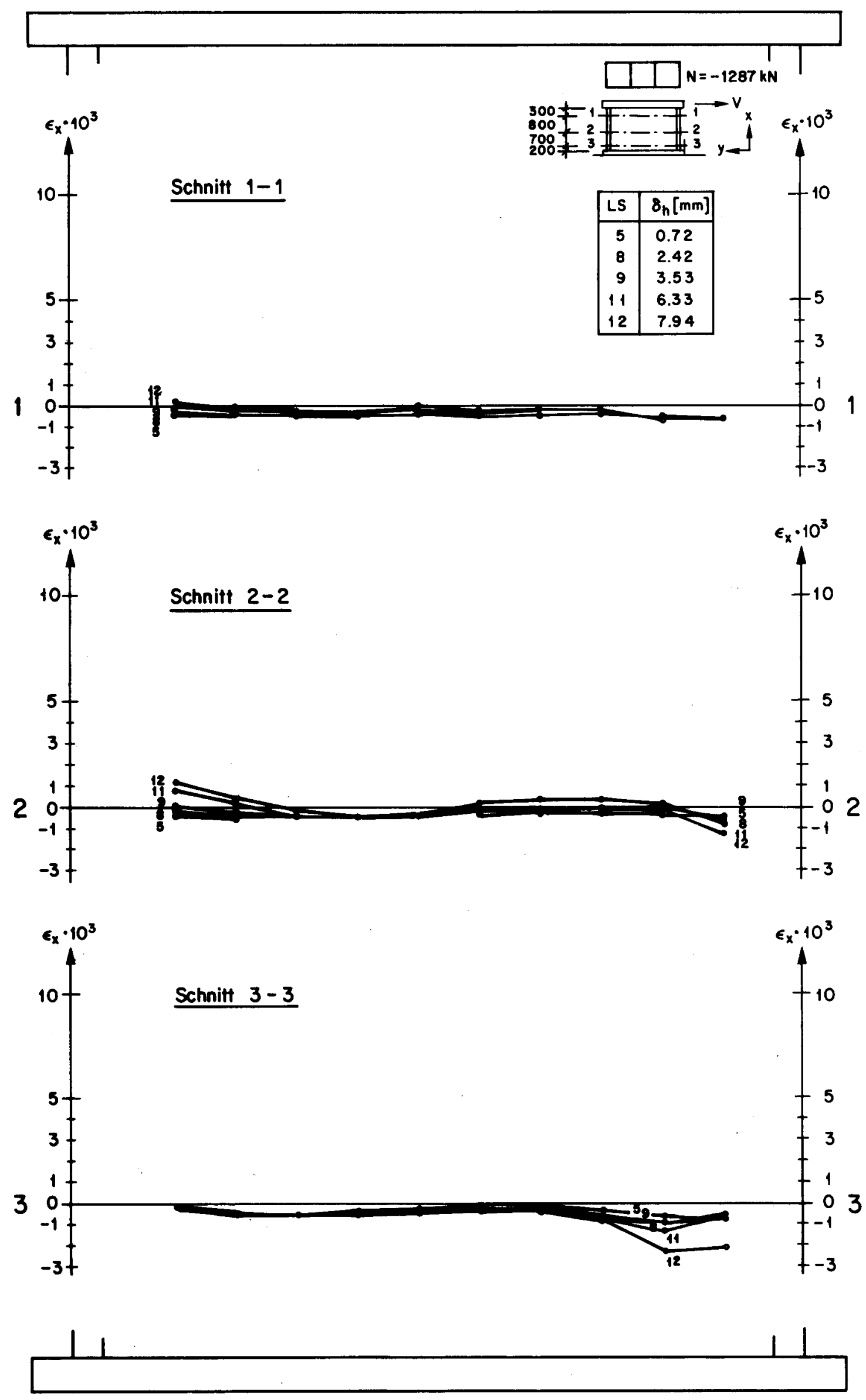

Bild 53: Dehnungen $\epsilon_{\mathrm{x}}, W 2$ 

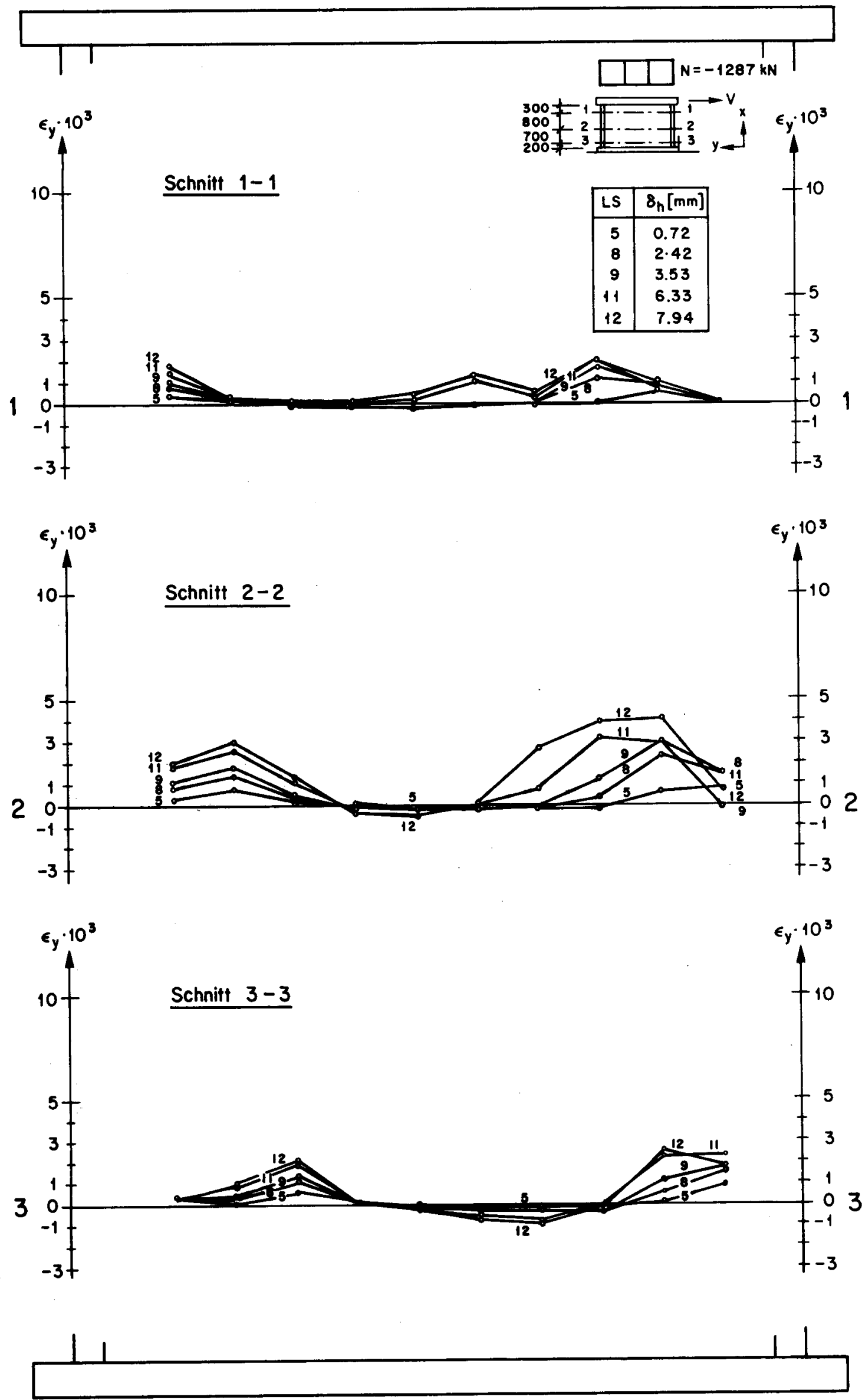

Bild 54: Dehnungen $\epsilon_{y}, W 2$ 

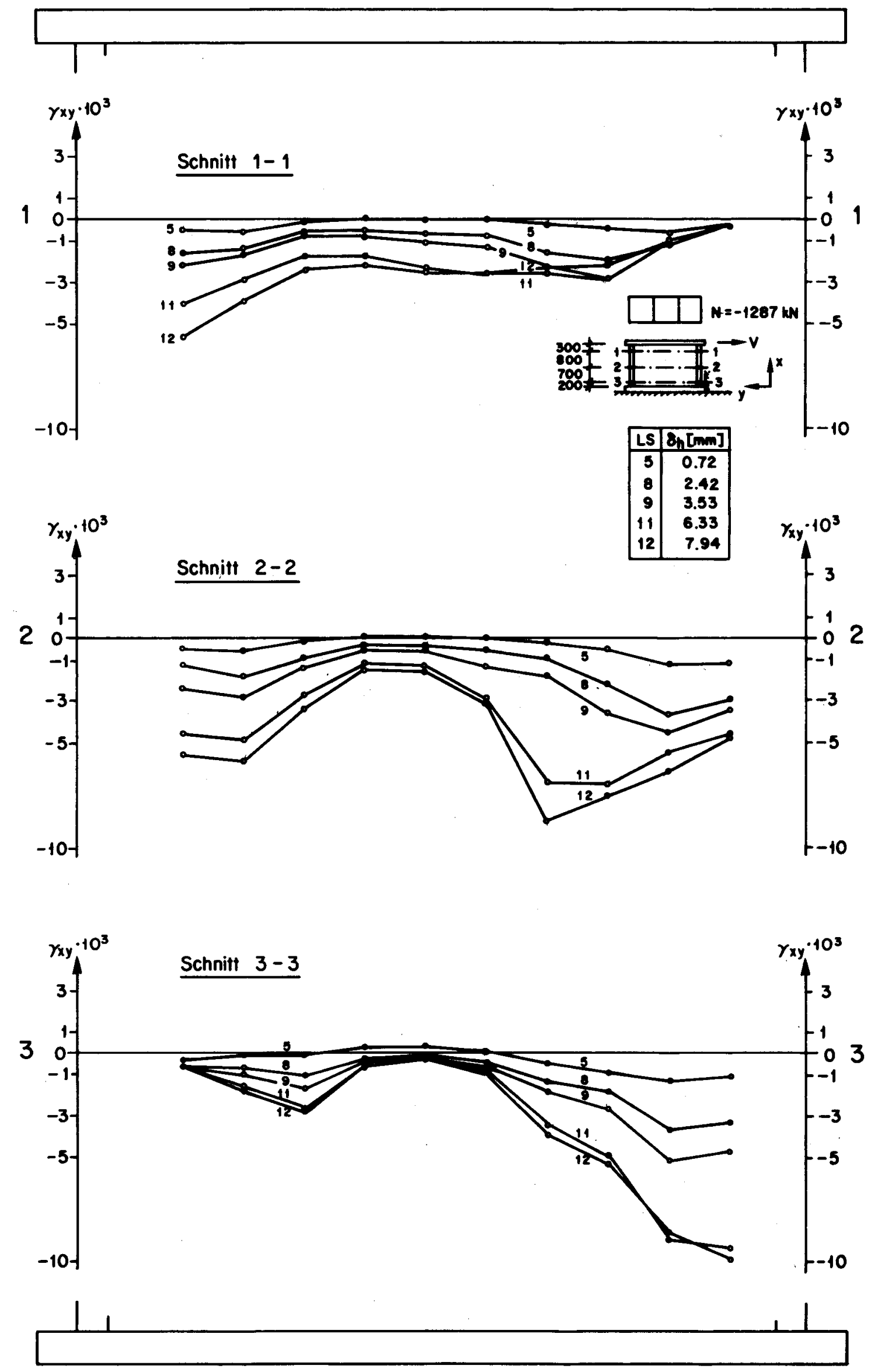

Bild 55: Schiebungen $\gamma_{x y}, W 2$ 

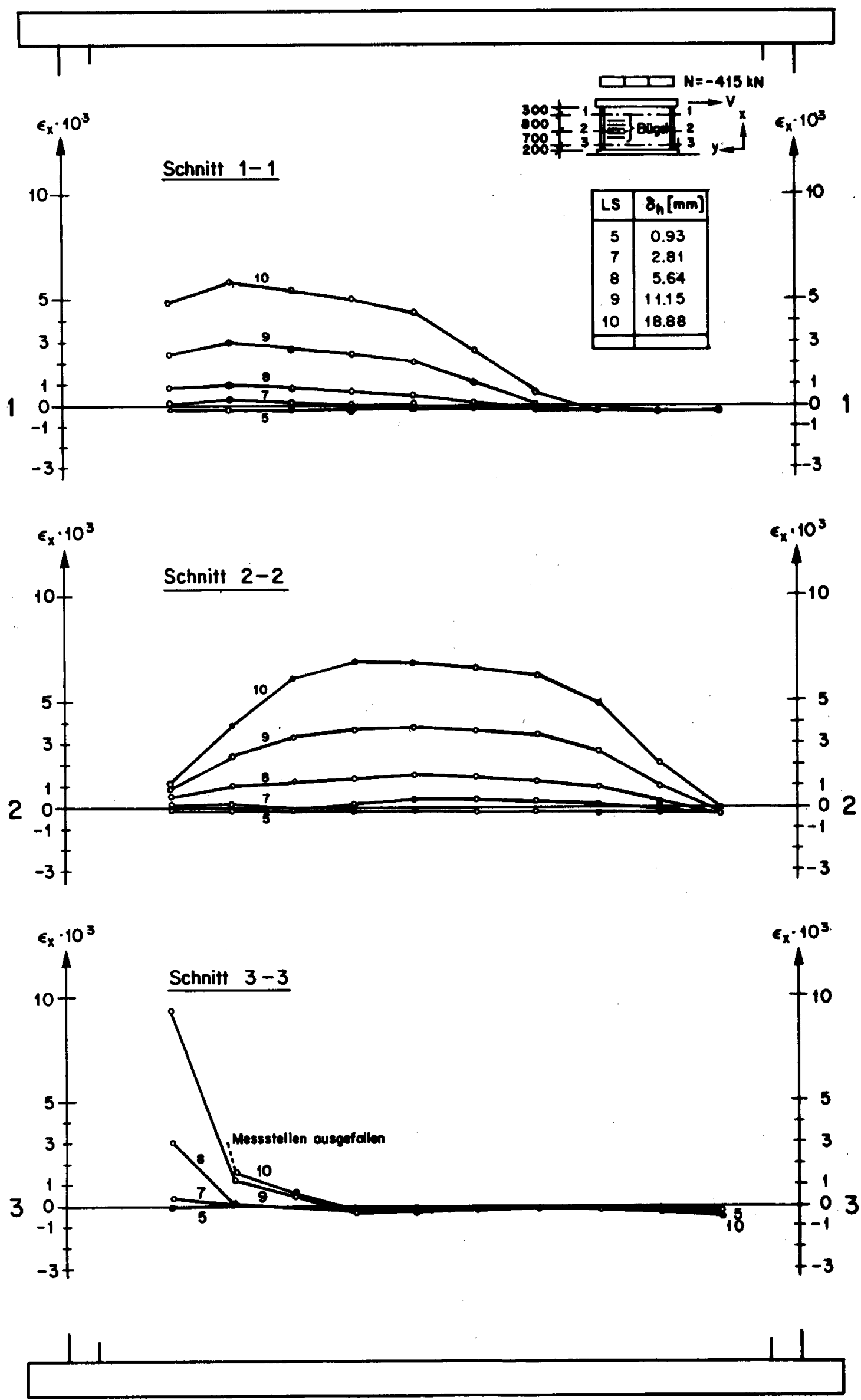

Bild 56: Dehnungen $\epsilon_{x}, W 3$ 

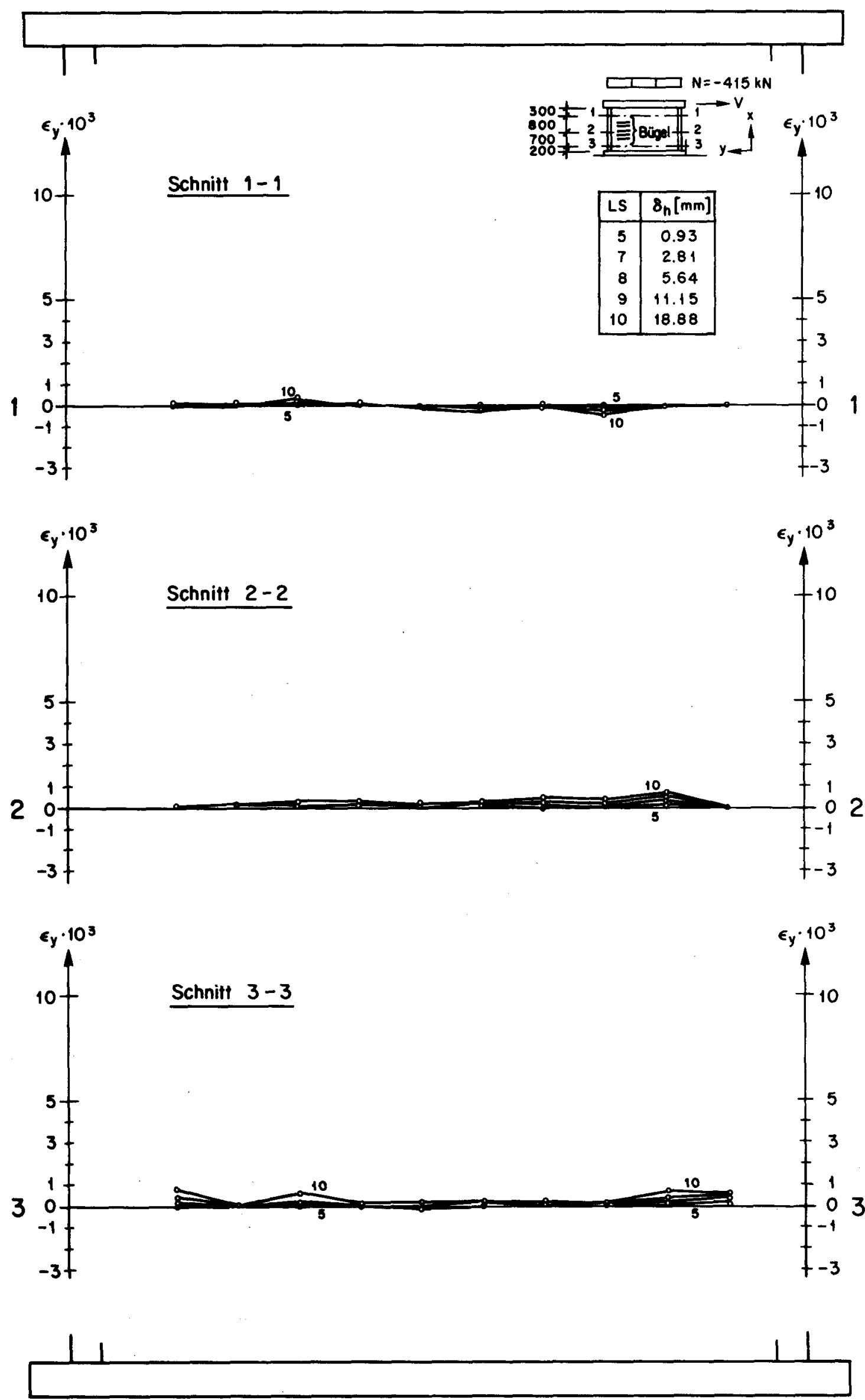

Bild 57: Dehnungen $\epsilon_{y}, W 3$ 

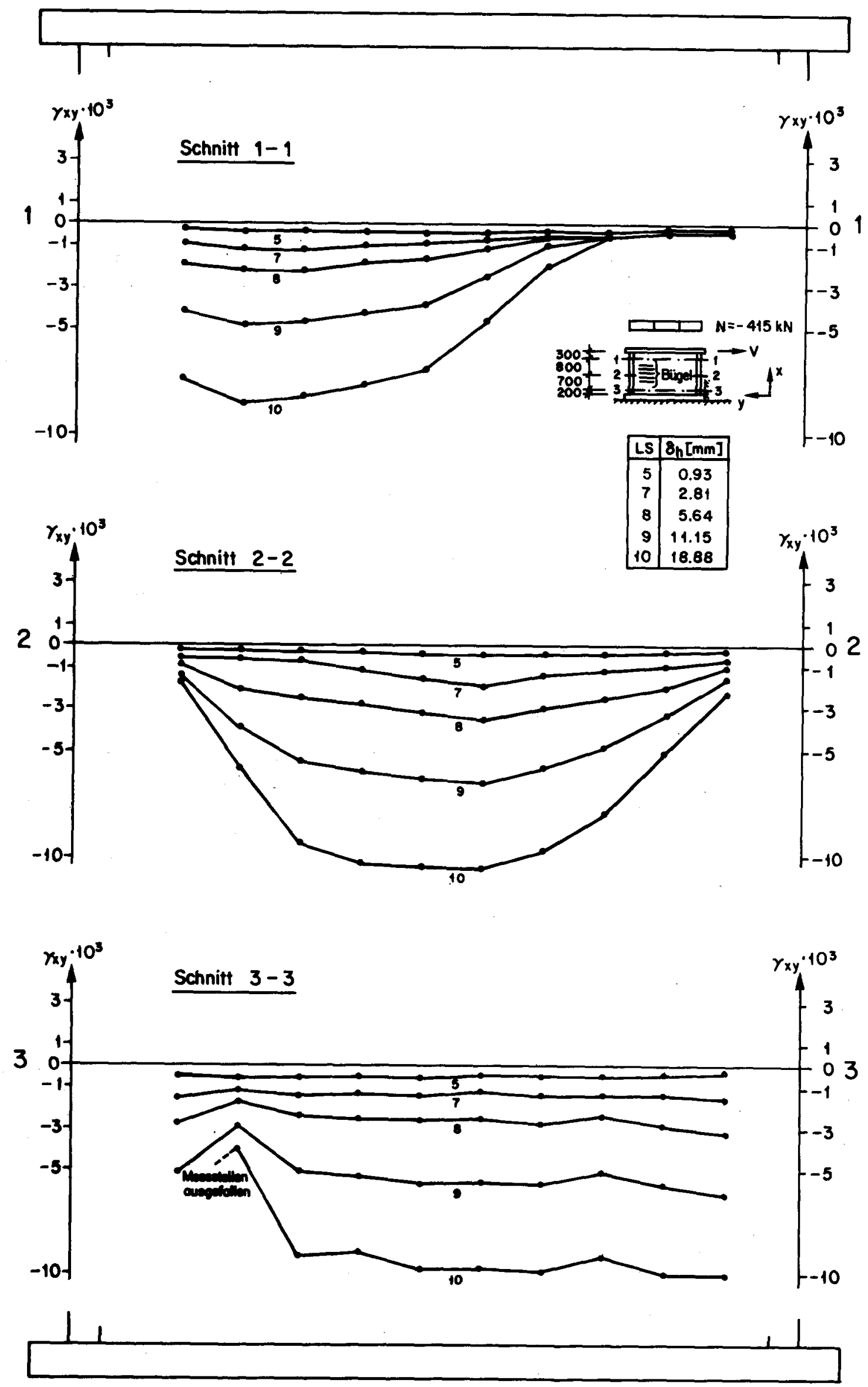

Bild 58: Schiebungen $\gamma_{x y}, w 3$ 

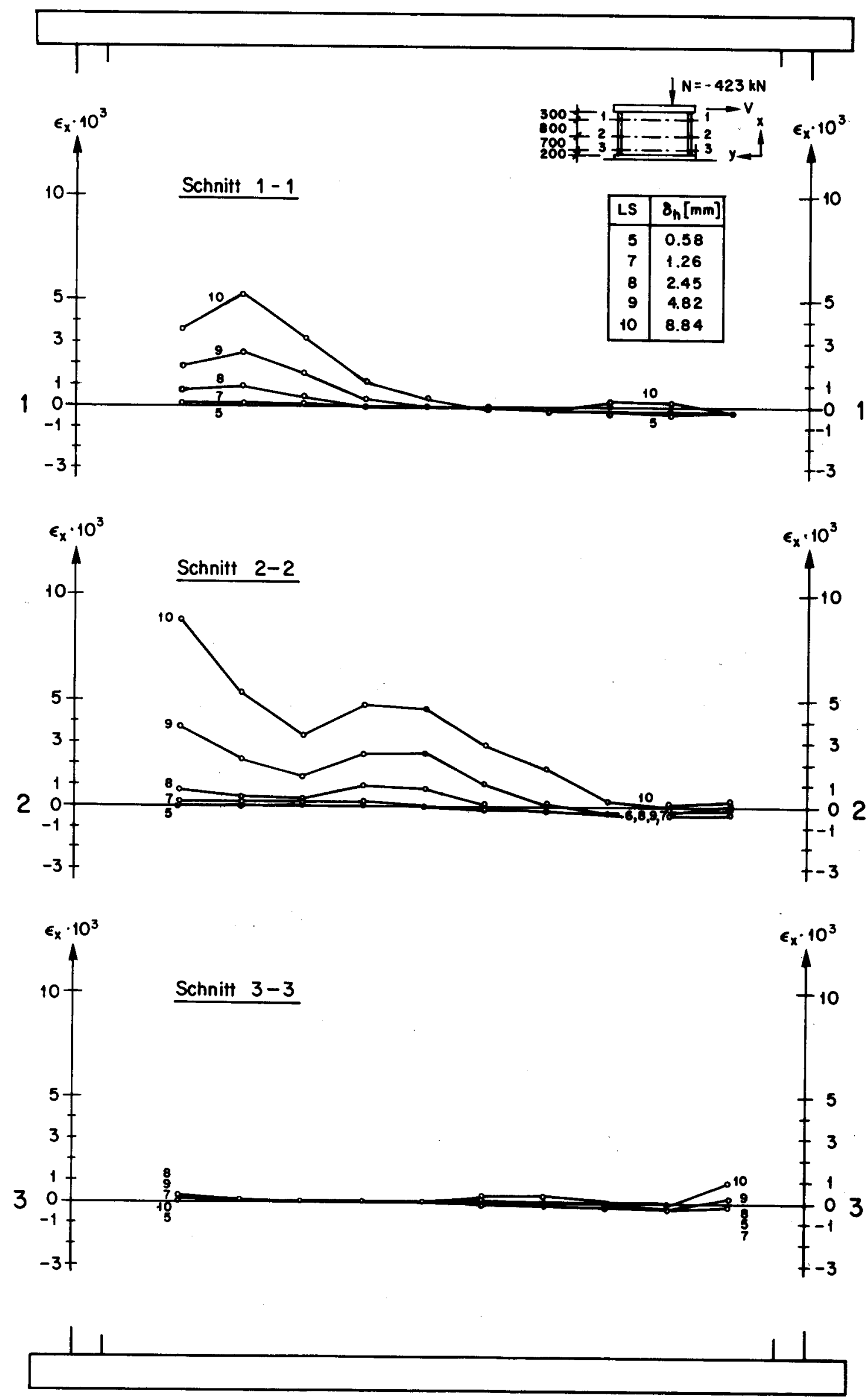

Bild 59: Dehnungen $\epsilon_{\mathrm{x}}, W_{4}$ 

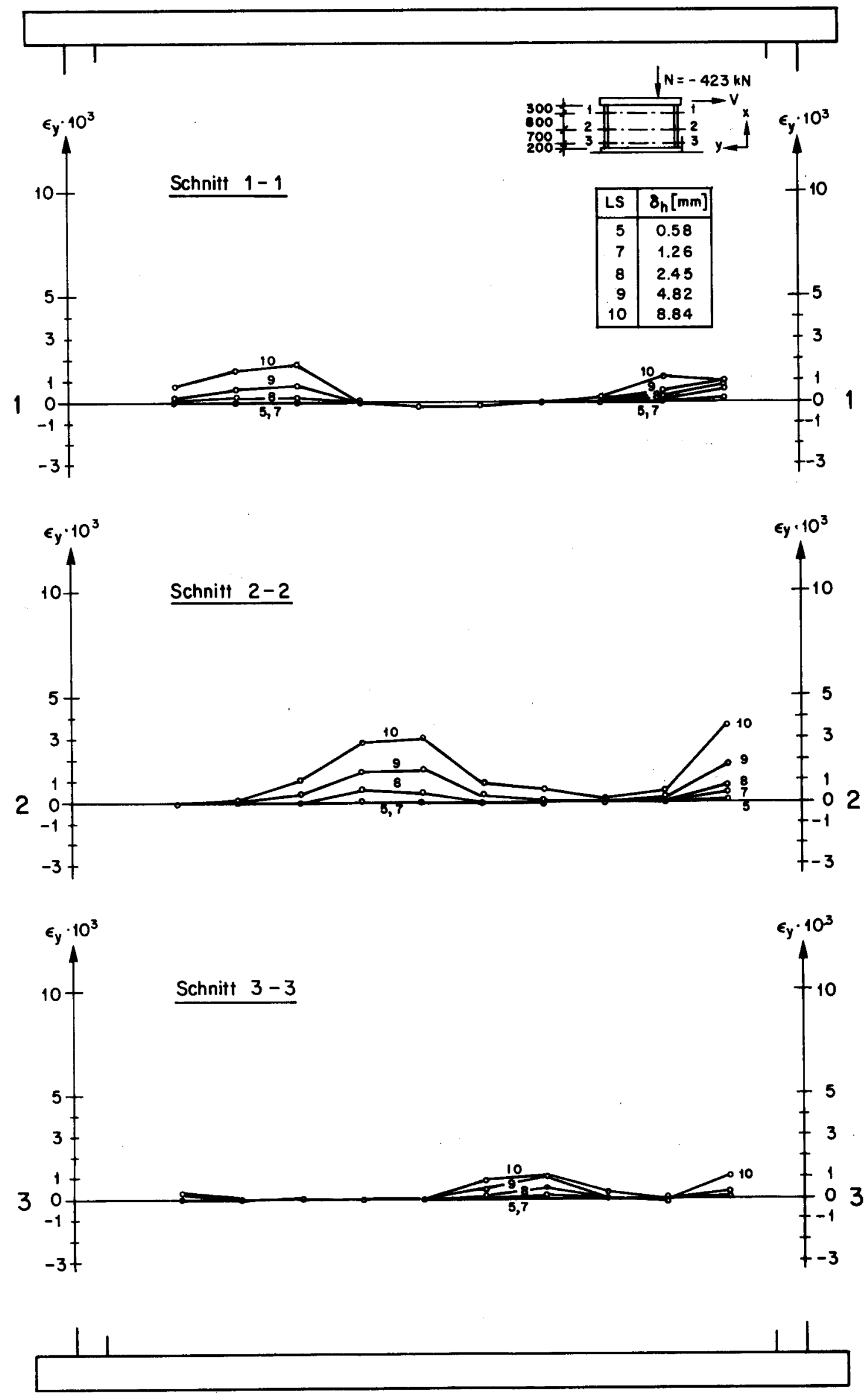

Bild 60: Dehnungen $\epsilon_{y}, W 4$ 

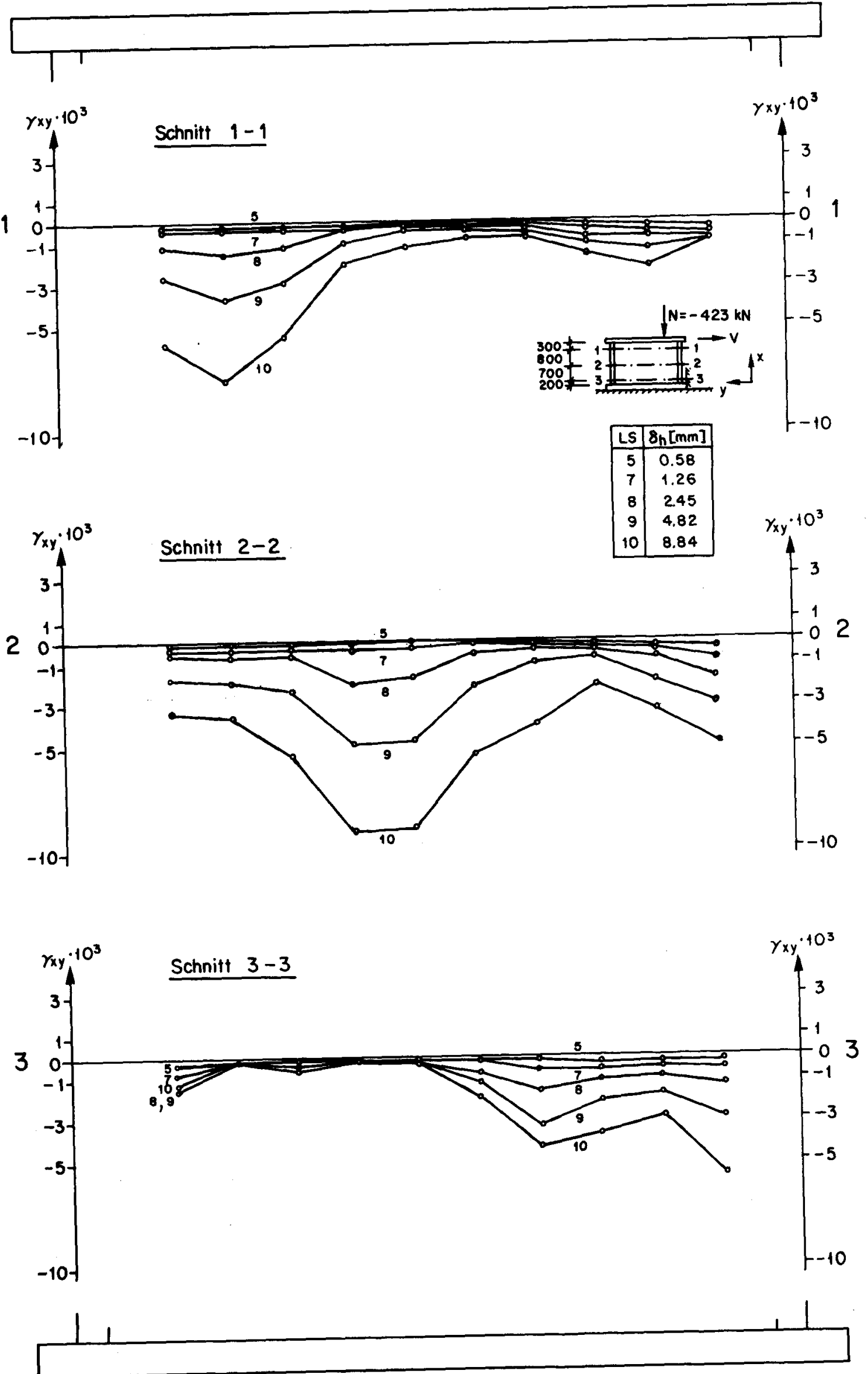

Bild 61: Schiebungen $\gamma_{x y}, w 4$ 

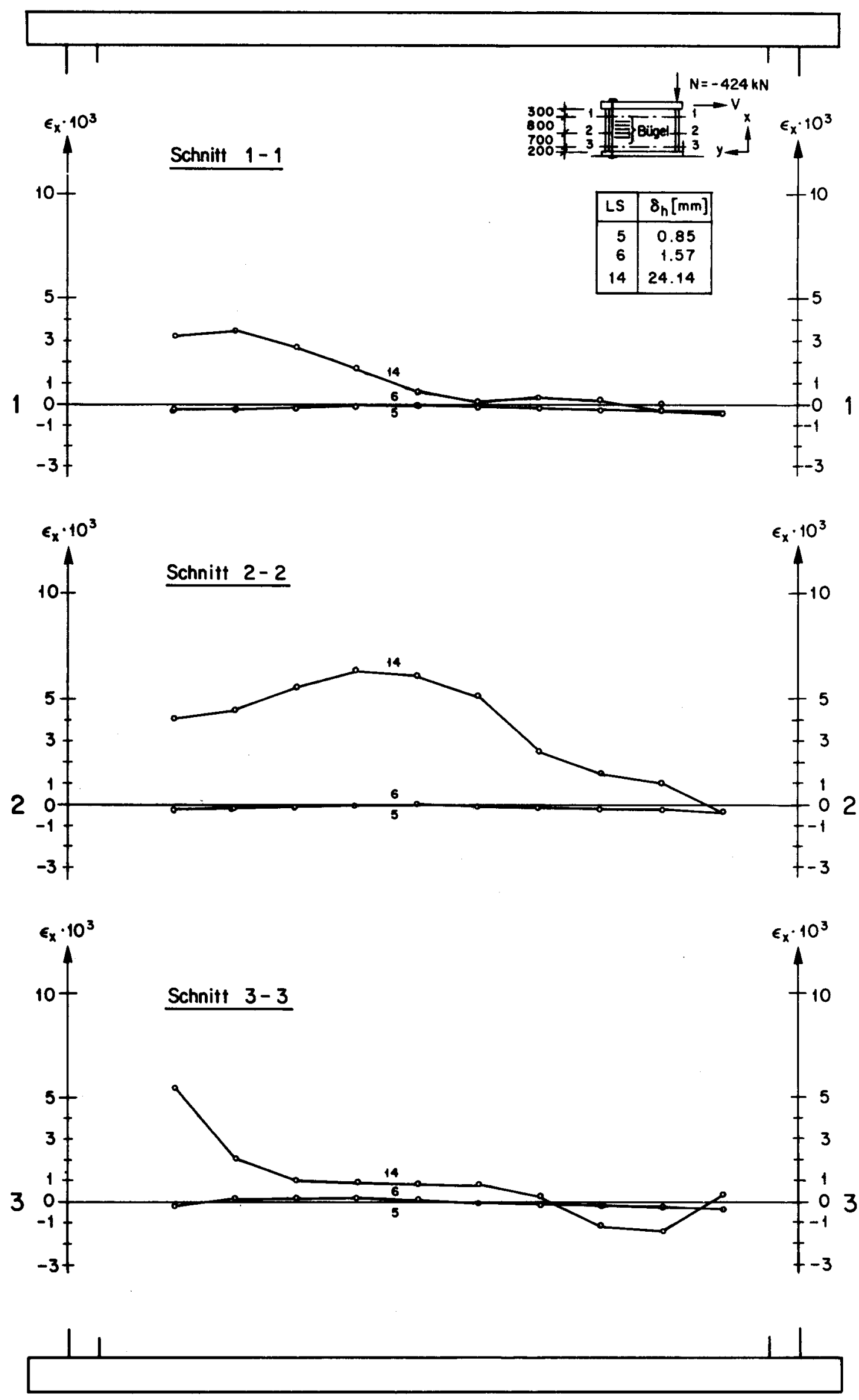

Bild 62: Dehnungen $\epsilon_{x}$, W 5 

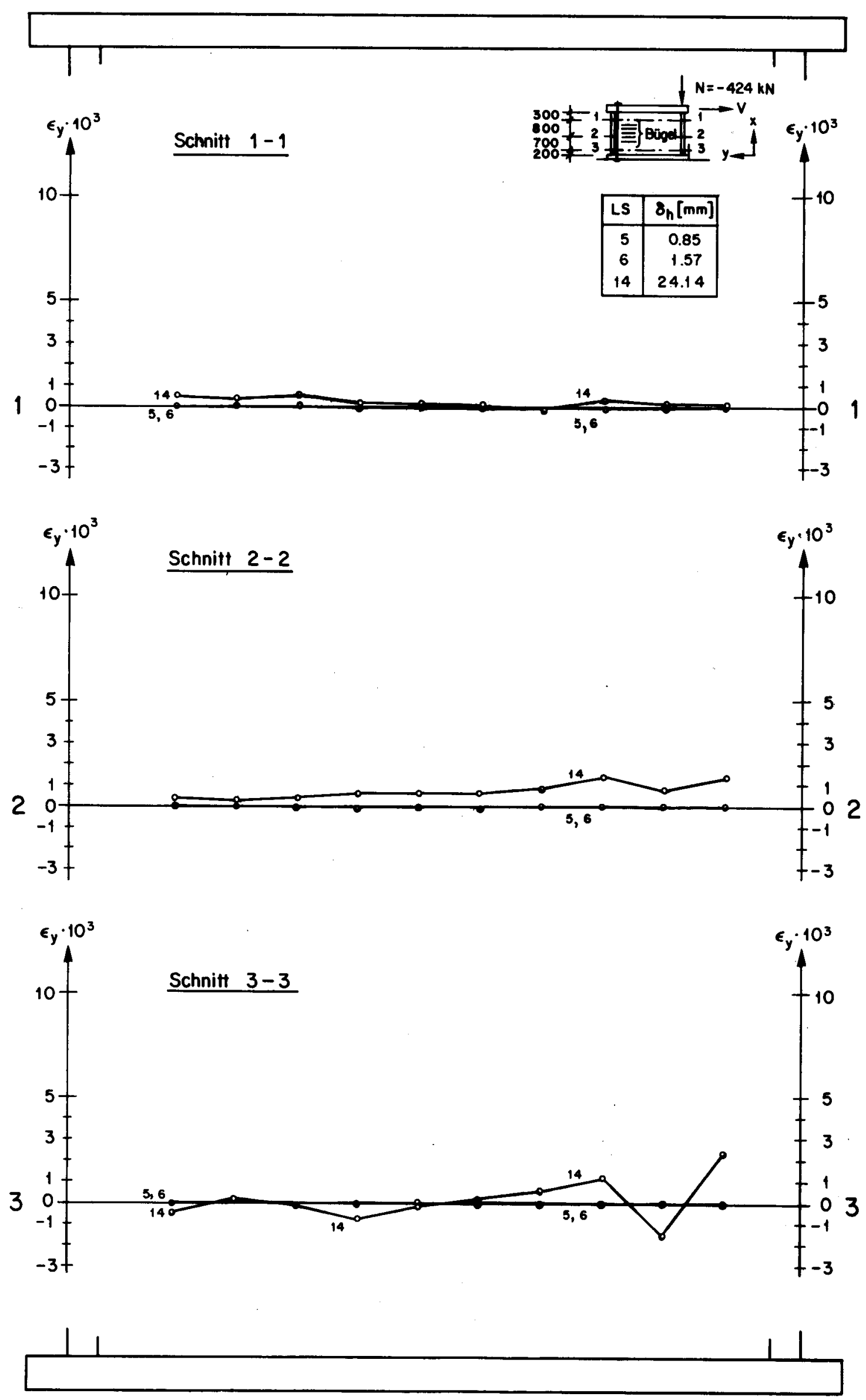

Bild 63: Dehnungen $\epsilon_{y}, W 5$ 

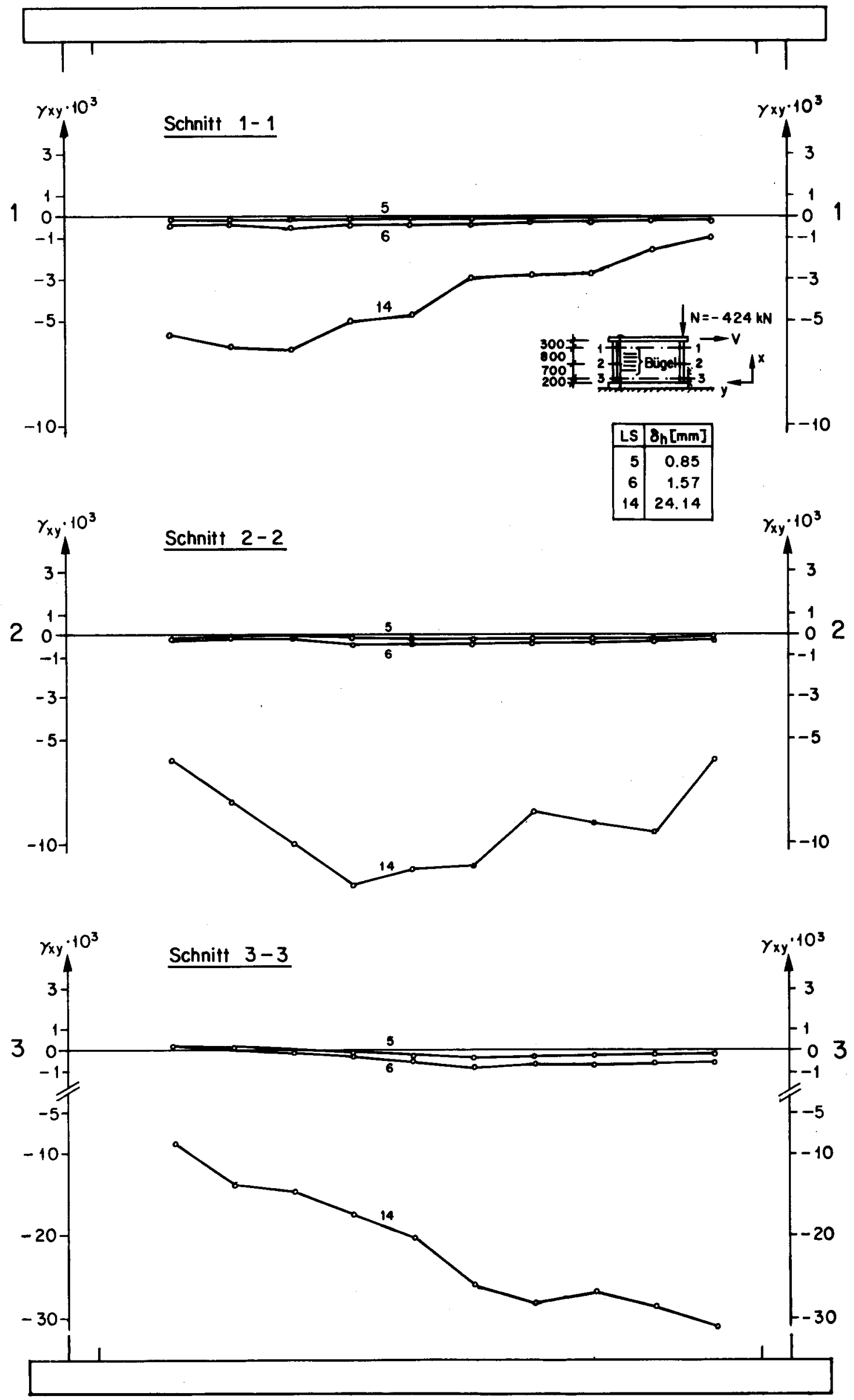

Bild 64: Schiebungen $\gamma_{x y}$, W 5 

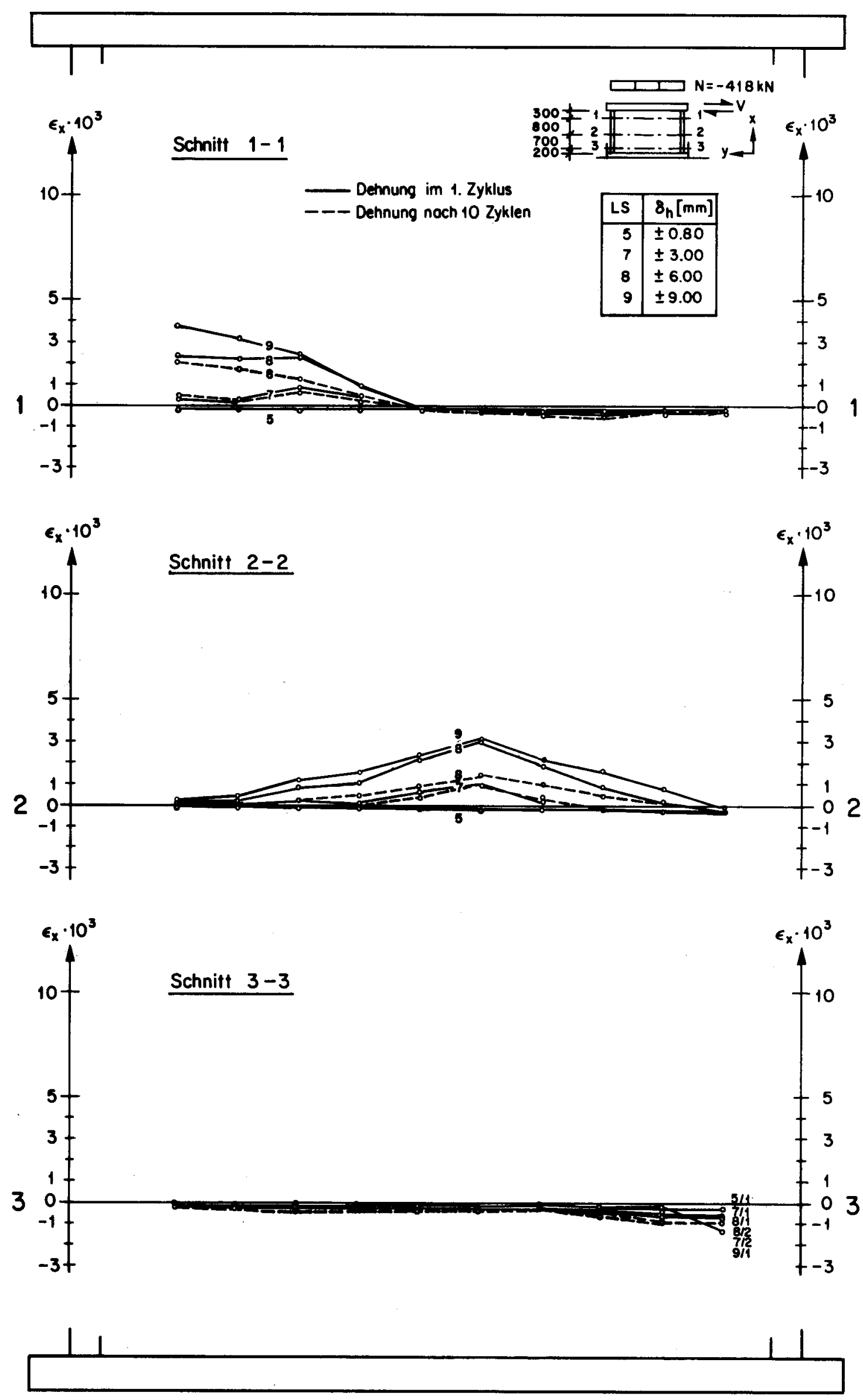

Bild 65; Dehnungen $\epsilon_{\mathrm{x}}, W 6$ 

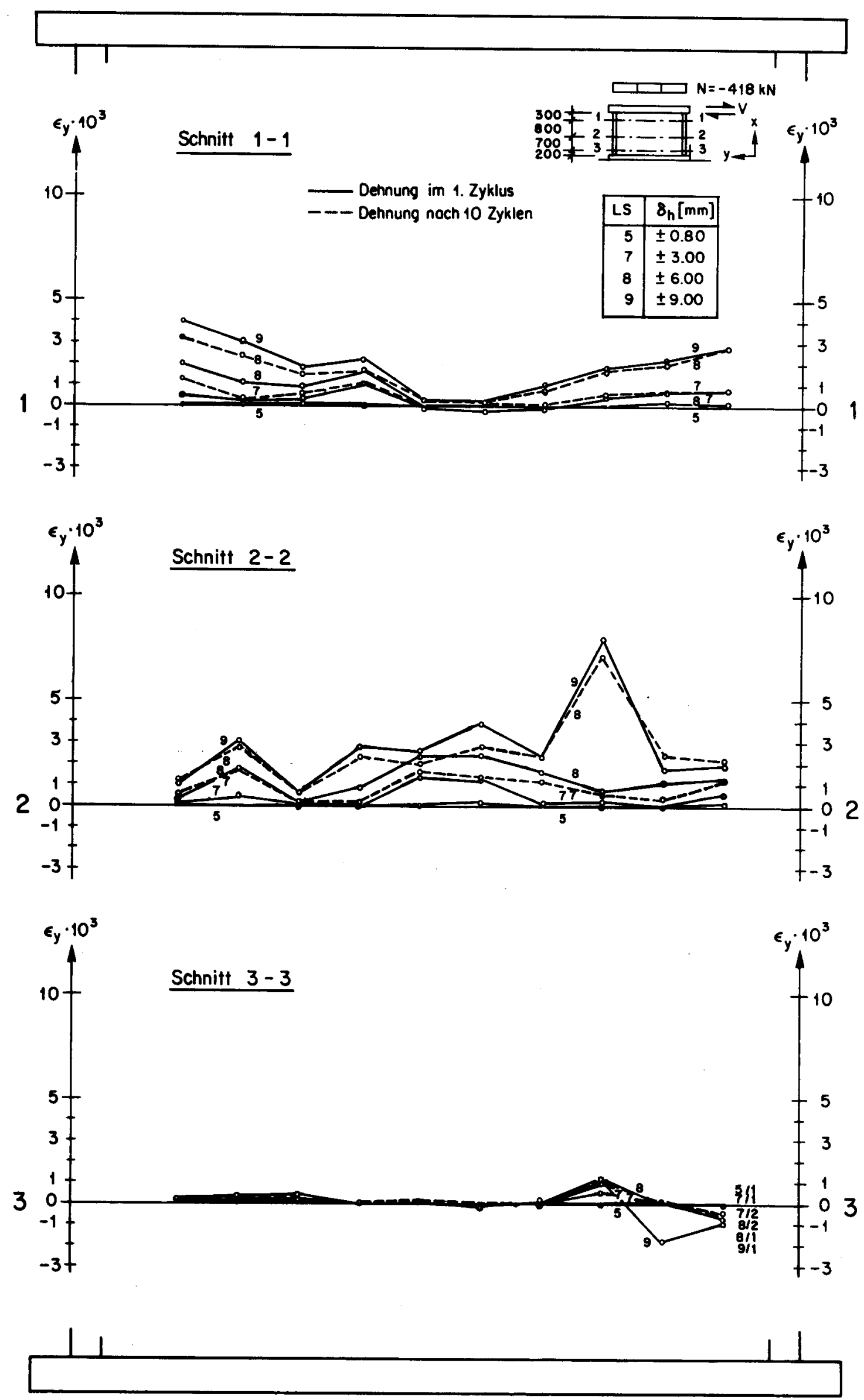

Bild 66: Dehnungen $\epsilon_{y}, W 6$ 

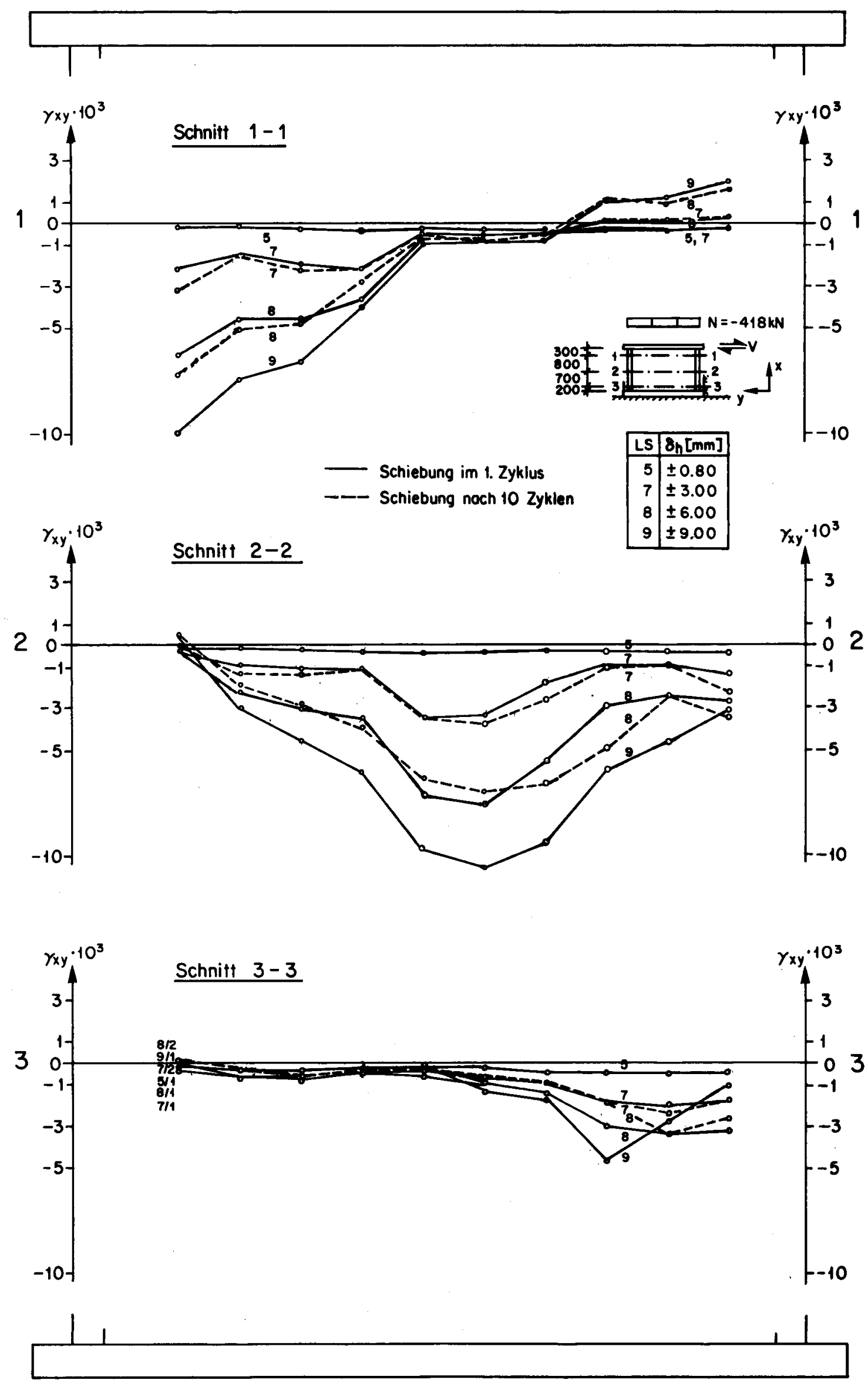

Bild 67: Schiebungen $\gamma_{x y}, W 6$ 

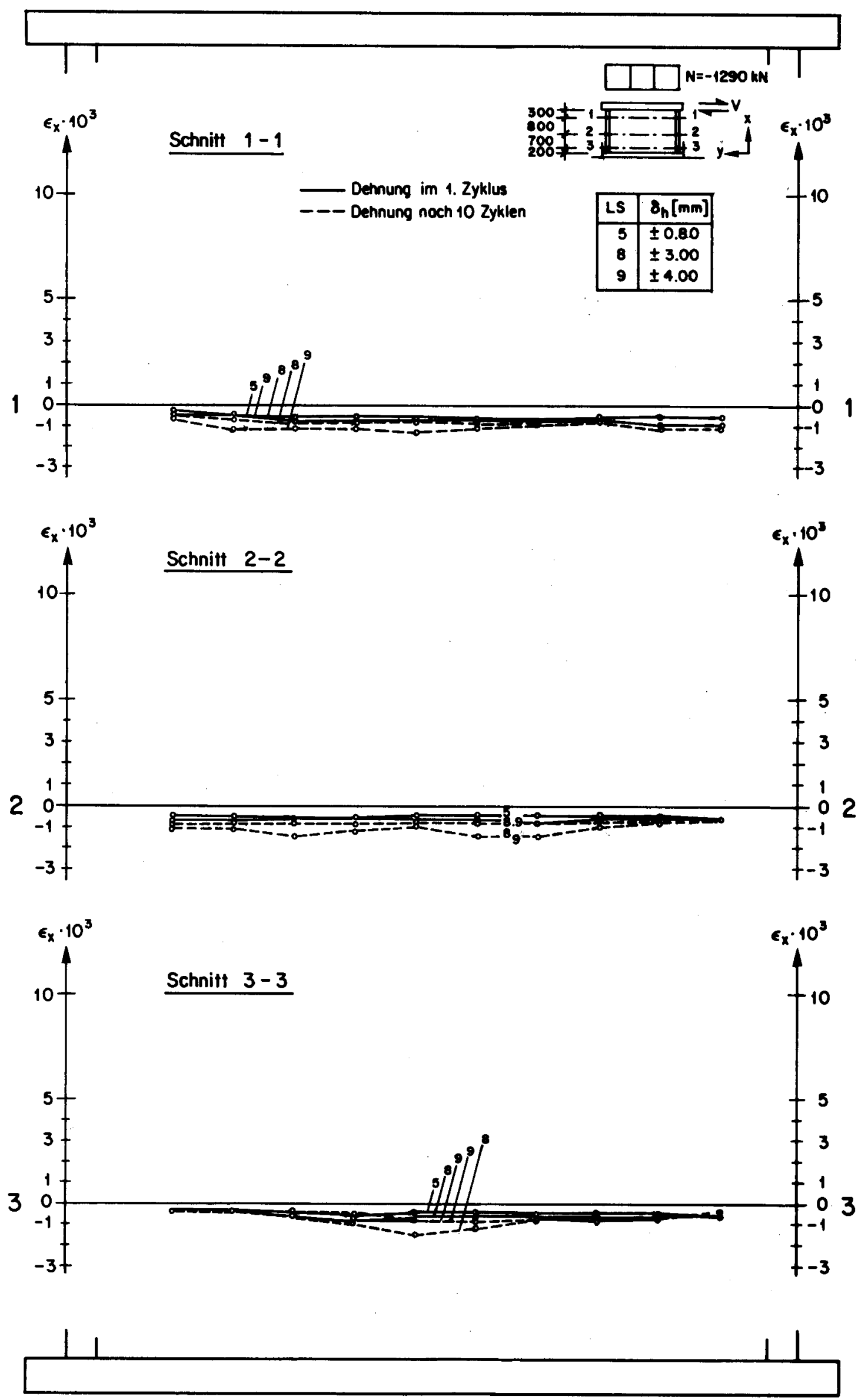

Bild 68: Dehnungen $\epsilon_{x}, W 7$ 

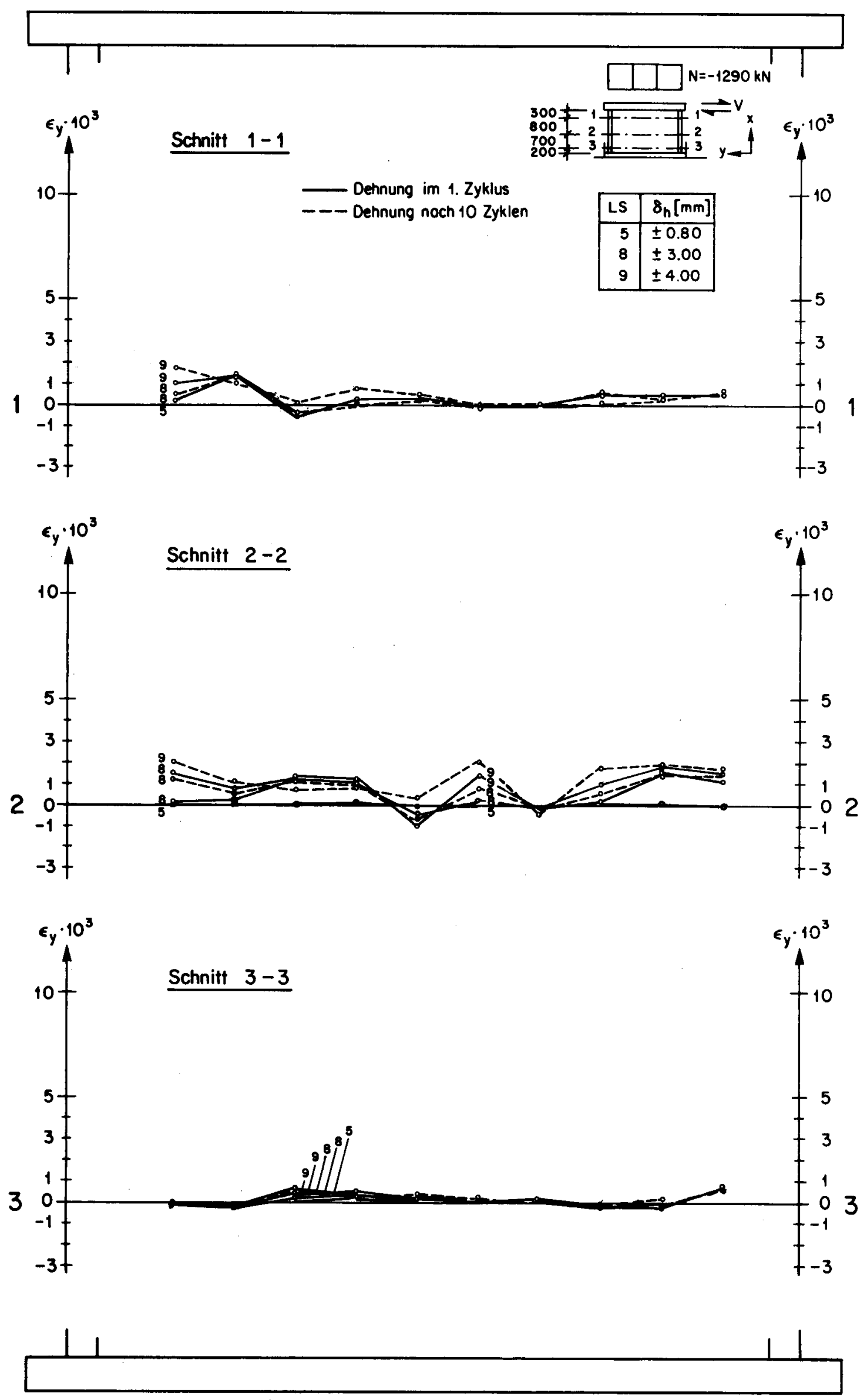

Bild 69: Dehnungen $\epsilon_{y}, W 7$ 

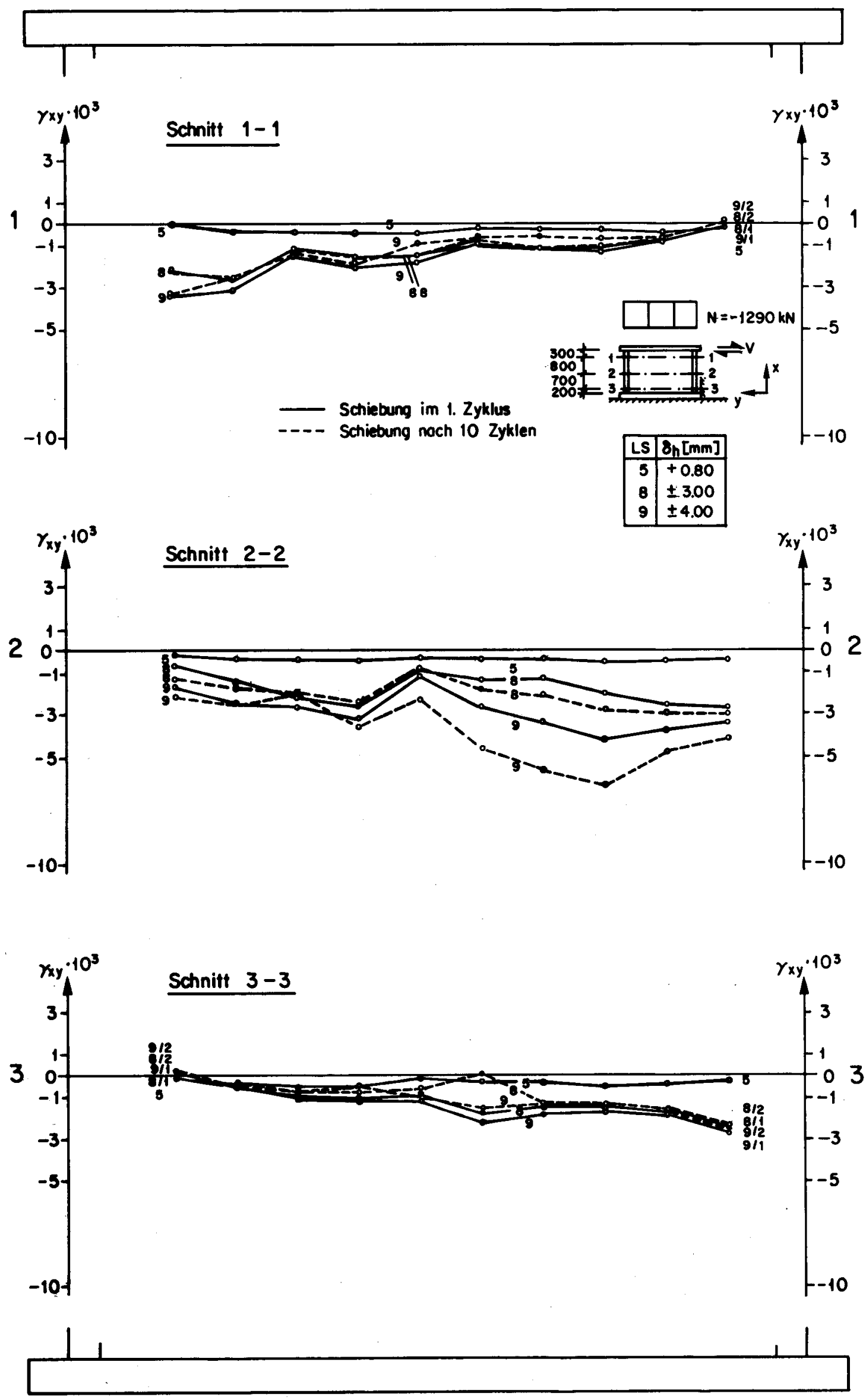

Bild 70: Schiebungen $\gamma_{x y}, w 7$ 


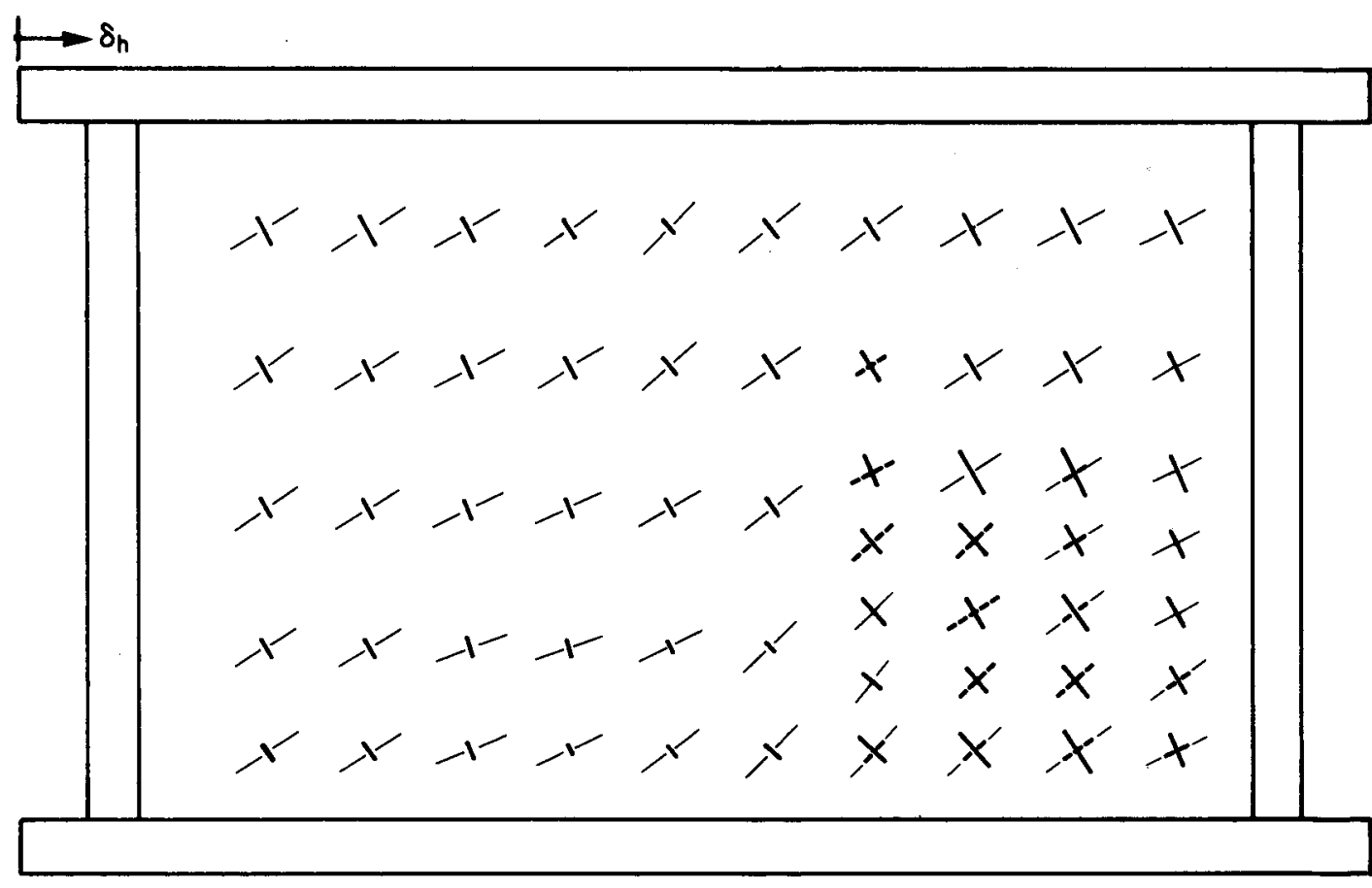

LS 10, $\quad \delta_{h}=0.83 \mathrm{~mm}$

Mossstob : $\longmapsto-1 \cdot 10^{-3}$

$1---1+1 \cdot 10^{-3}$

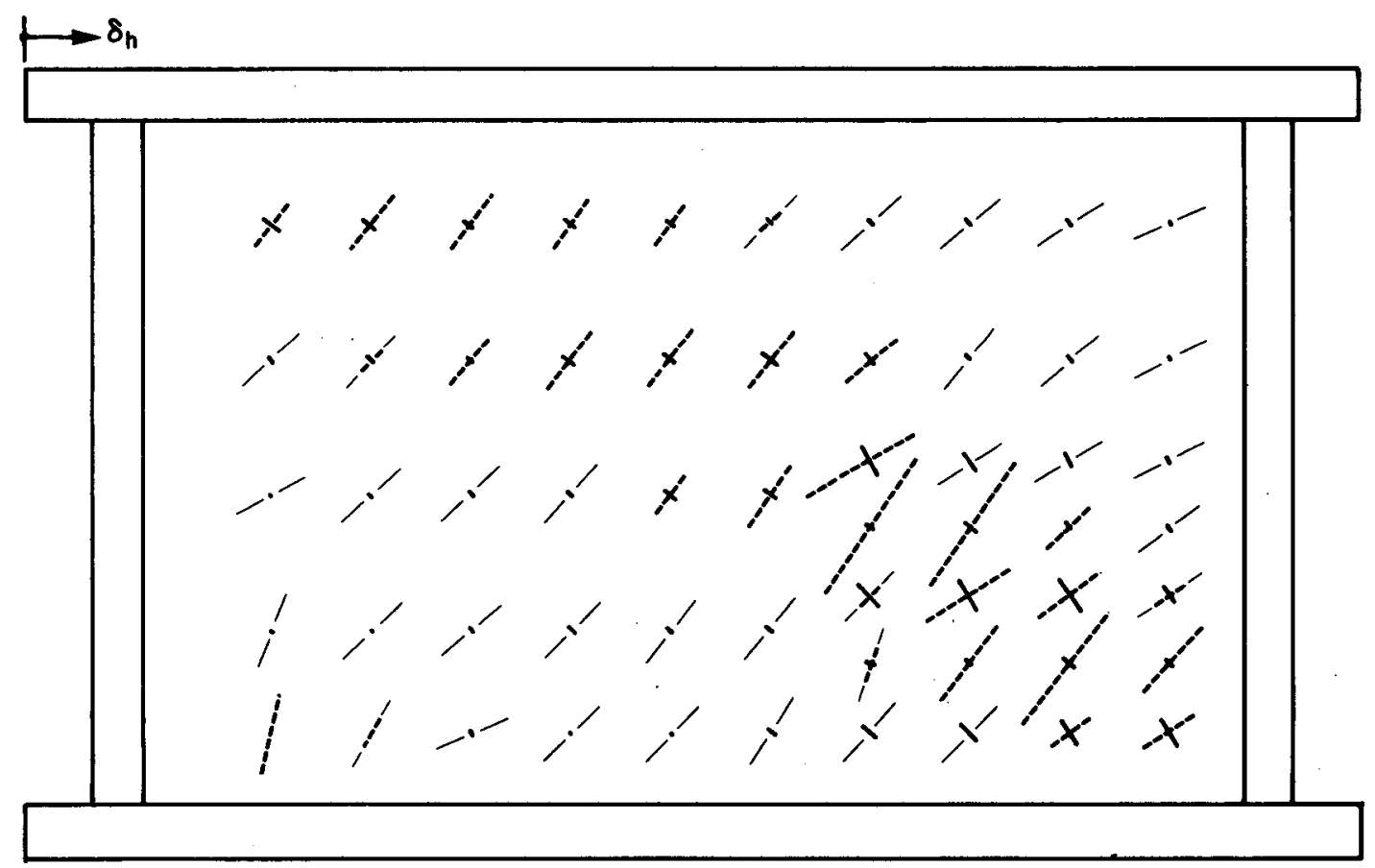

LS 21, $\quad \delta_{h}=7.59 \mathrm{~mm}$

Massstob : $\longmapsto-10 \cdot 10^{-3}$

$1--1+10 \cdot 10^{-3}$

Bild 71: Hauptdehnungen W 1 


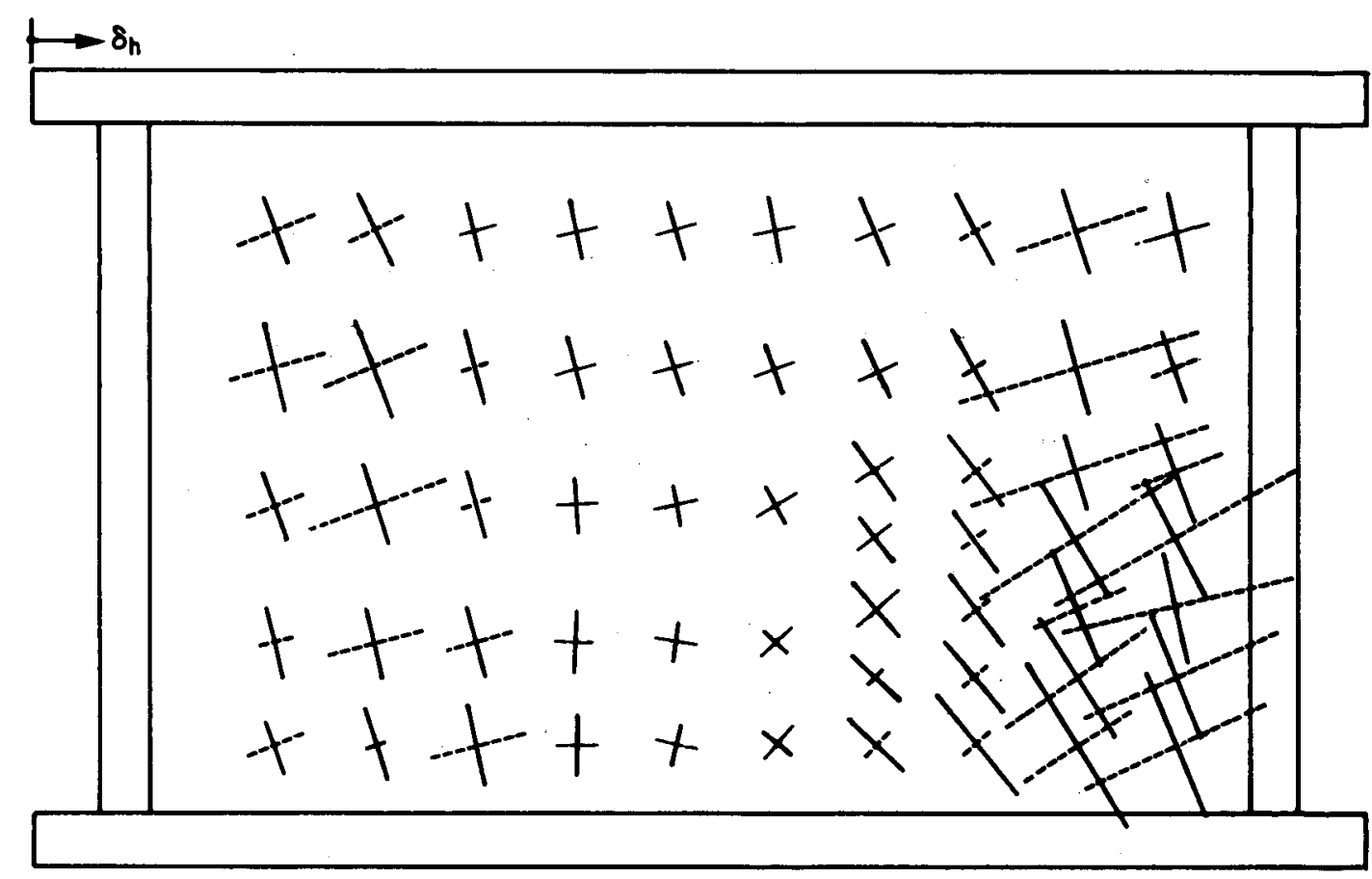

LS 6, $\delta_{h}=1.17 \mathrm{~mm} \quad$ Massstab : $\begin{aligned} \longmapsto & -1 \cdot 10^{-3} \\ & \longmapsto-1+1 \cdot 10^{-3}\end{aligned}$

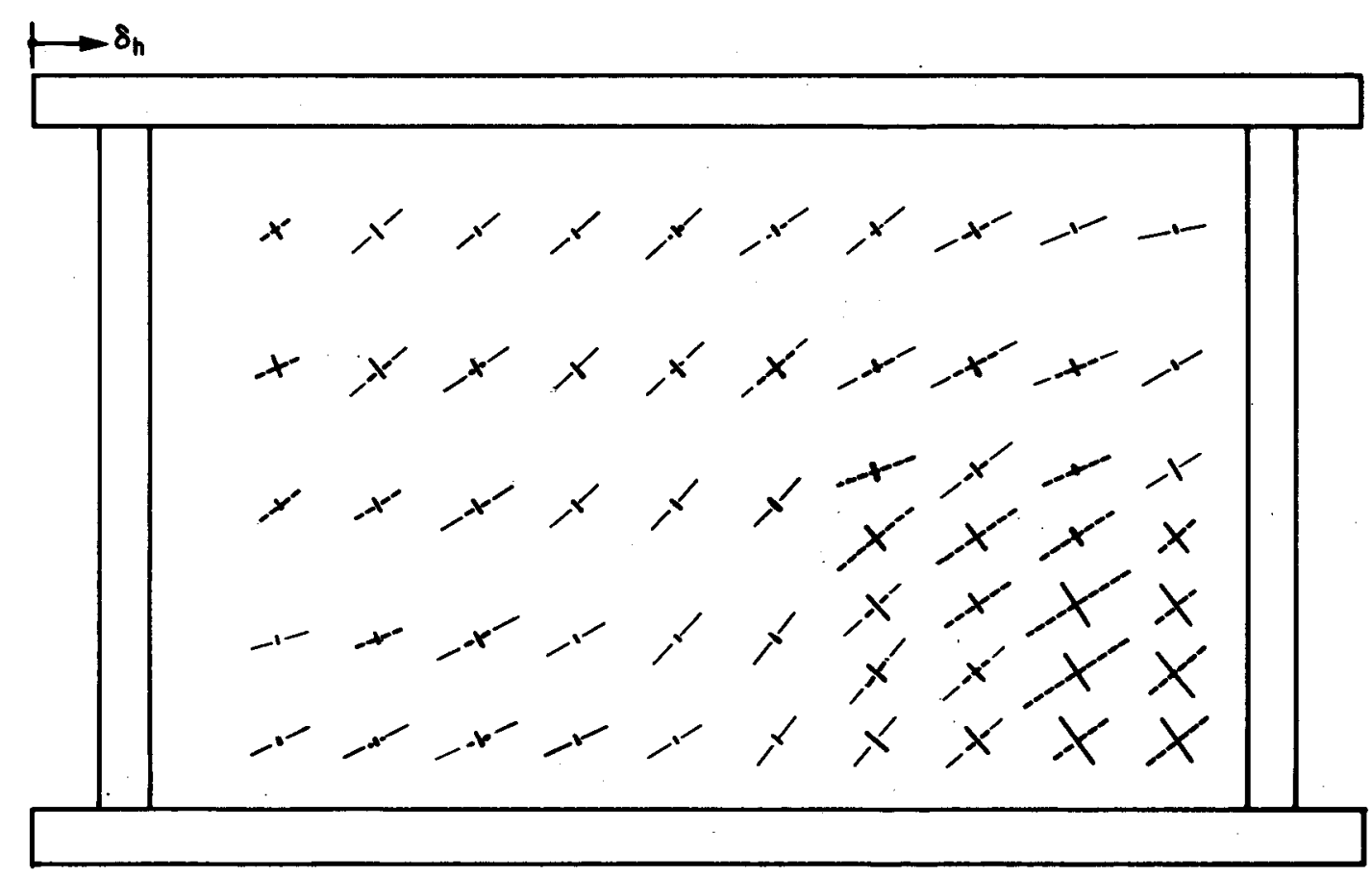

LS $11, \delta_{h}=6.33 \mathrm{~mm}$

Mossstob : $\begin{array}{r}\longmapsto-10 \cdot 10^{-3} \\ \longmapsto--10 \cdot 10^{-3}\end{array}$

Bild 72 : Hauptdehnungen W2 


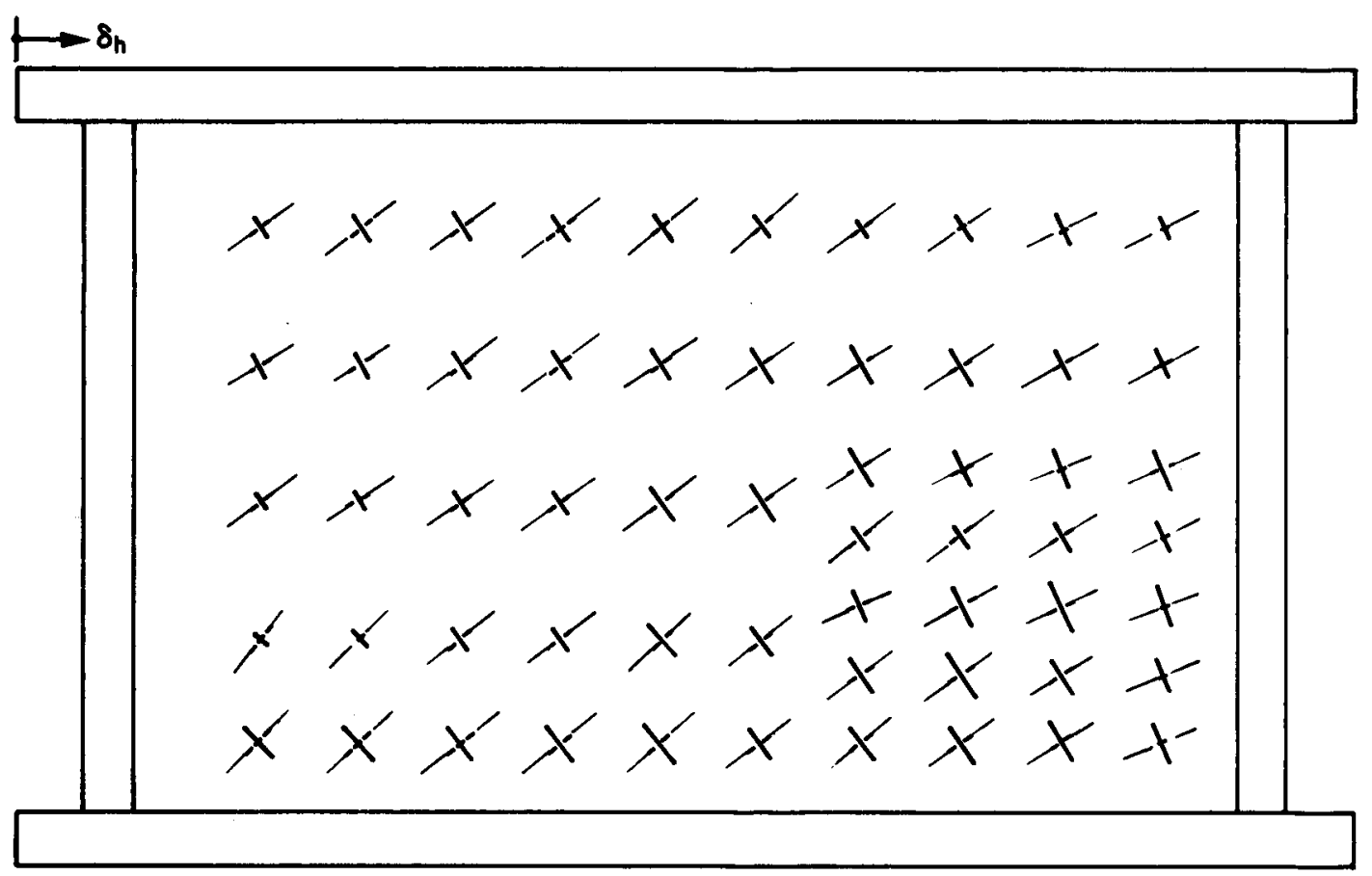

LS 5, $\delta_{h}=0.93 \mathrm{~mm}$

Massstab : $\longmapsto-1 \cdot 10^{-3}$

$1---1+1 \cdot 10^{-3}$

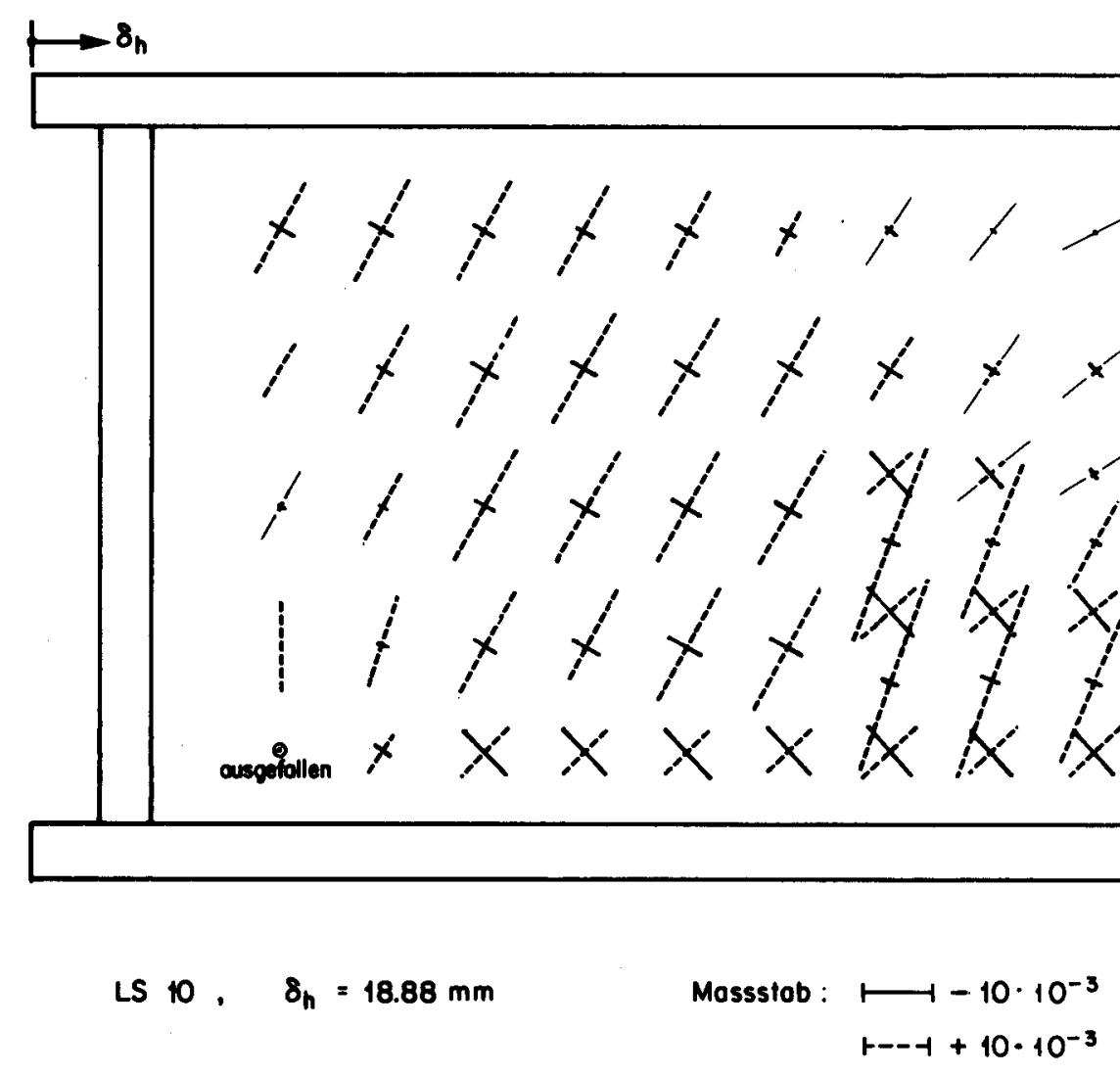

Bild 73: Hauptdehnungen W 3 


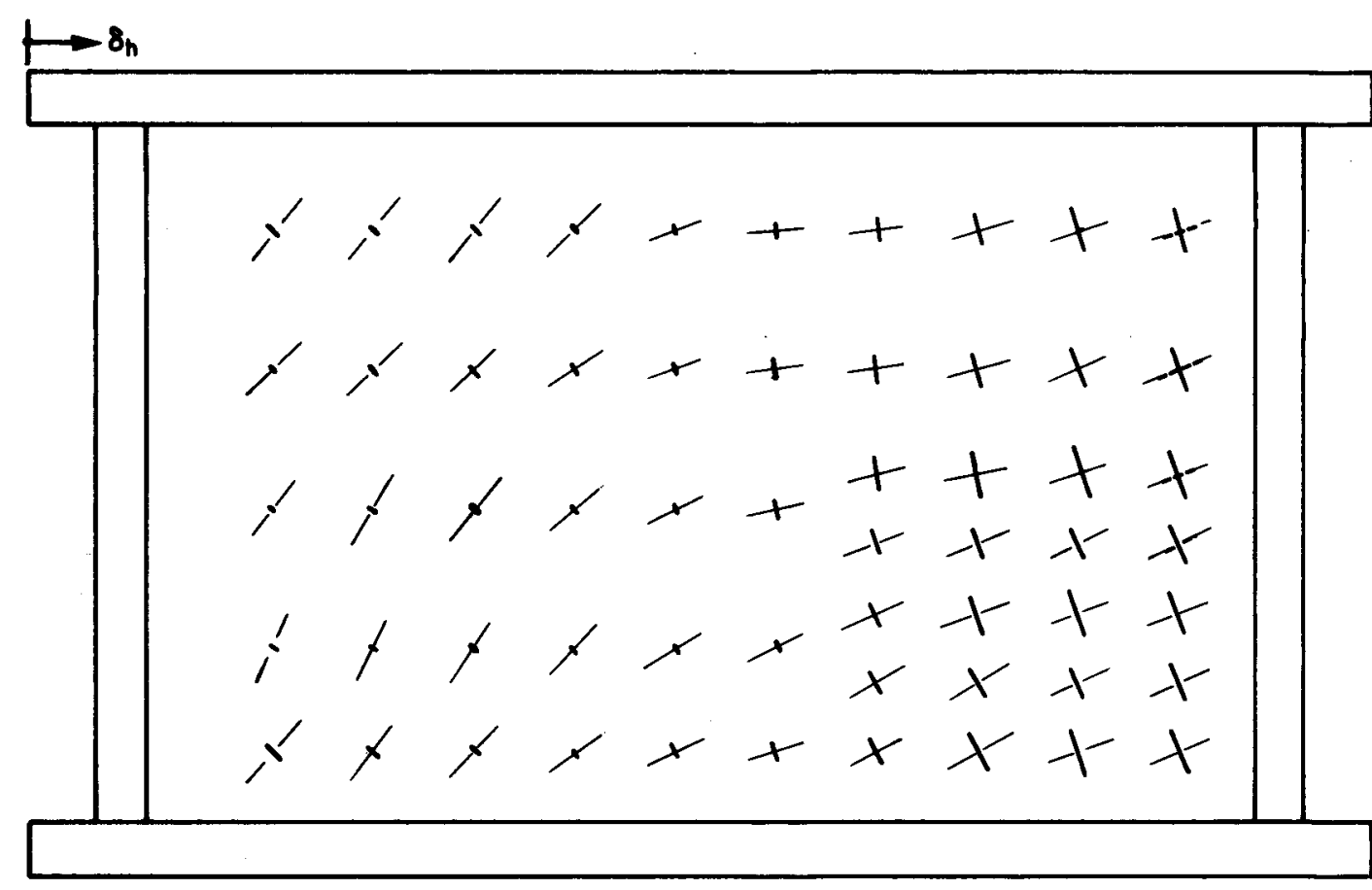

LS $5, \quad \delta_{h}=0.58 \mathrm{~mm}$

Mossstab : $\longmapsto-1 \cdot 10^{-3}$

$1---1+1 \cdot 10^{-3}$

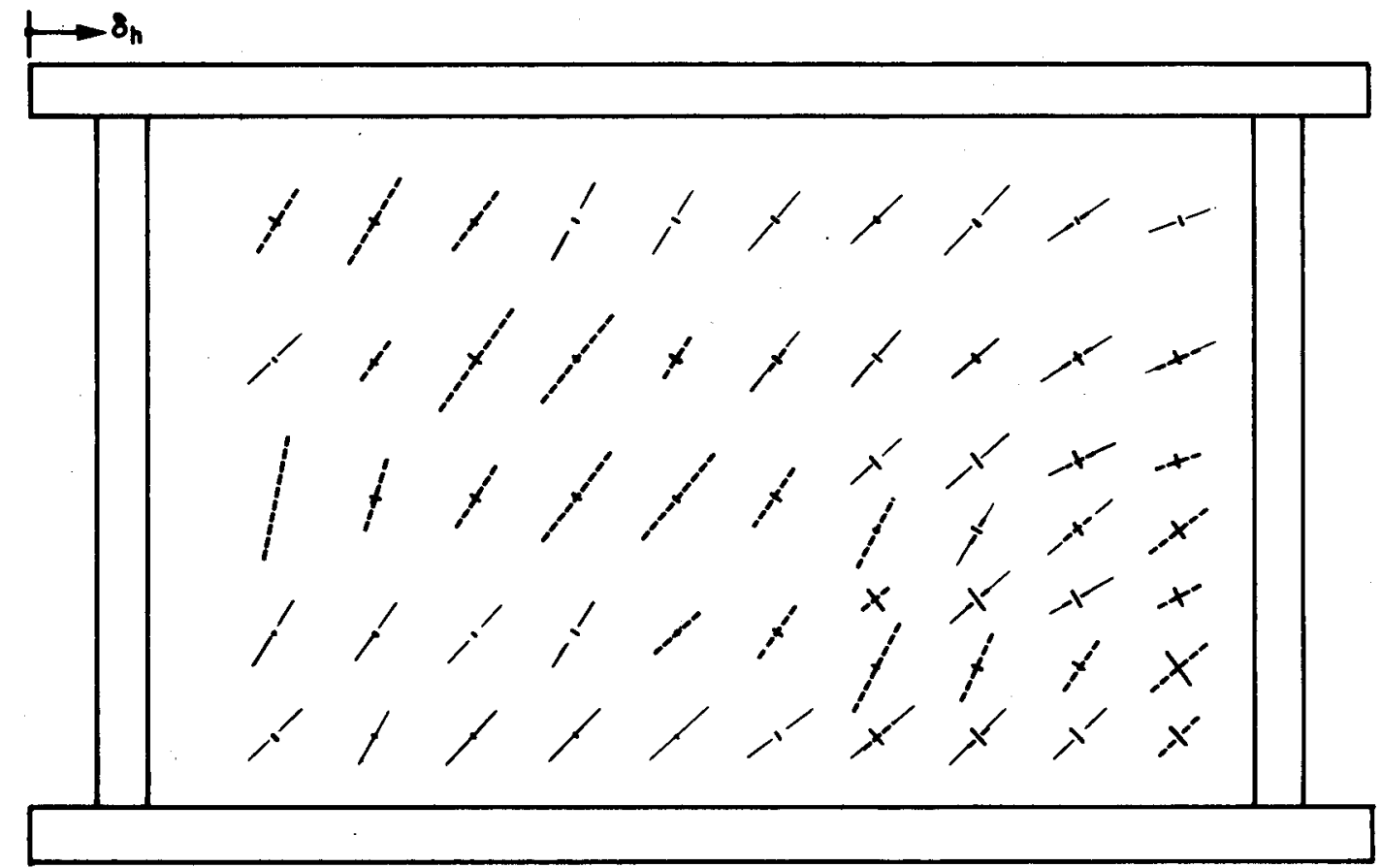

LS $10, \quad \delta_{h}=8.84 \mathrm{~mm}$

Mossstob : $\longmapsto-10 \cdot 10^{-3}$

$1--1+10 \cdot 10^{-3}$

Bild 74 : Hauptdehnungen W4 


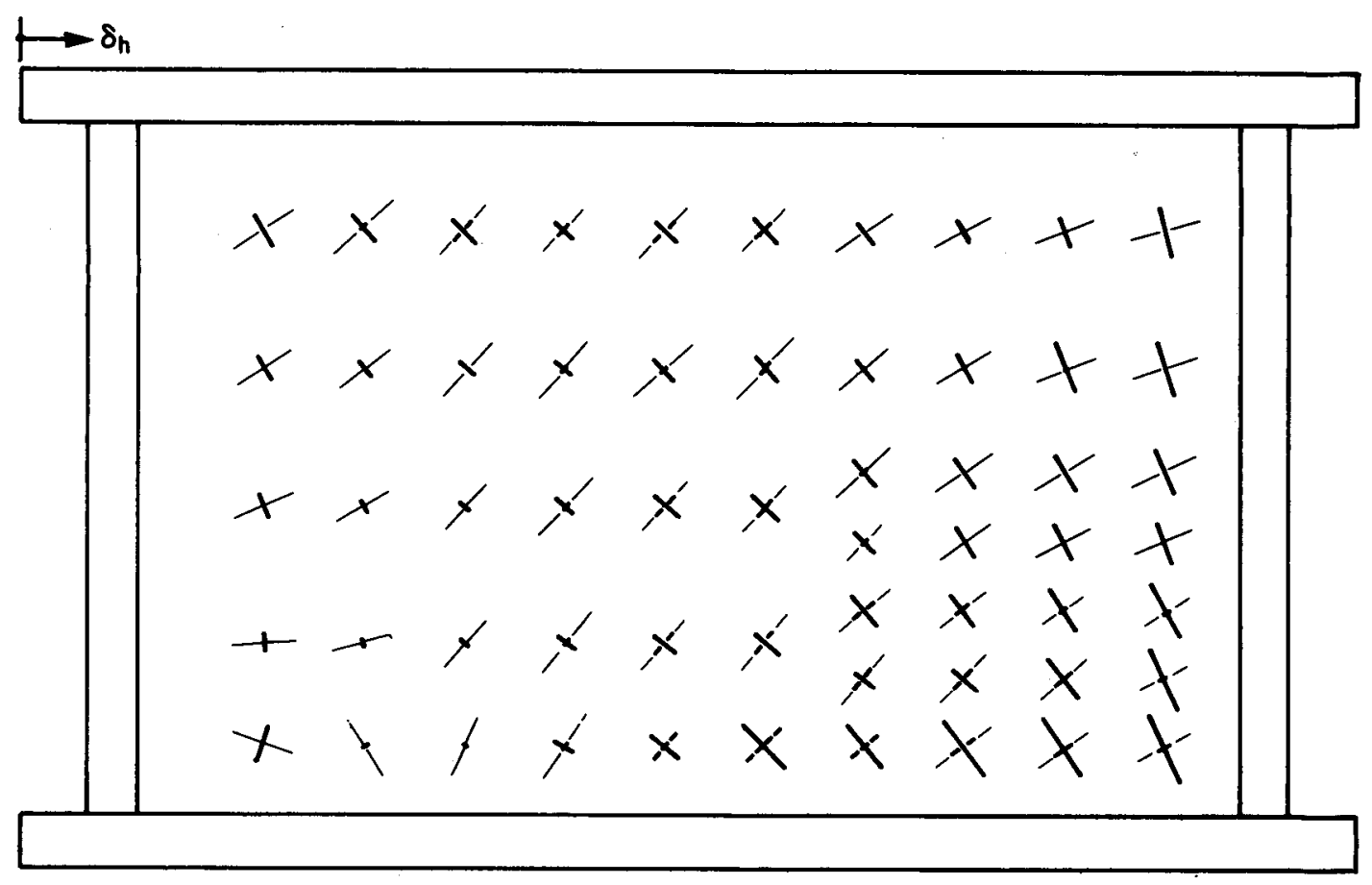

LS $6, \quad \delta_{h}=1.57 \mathrm{~mm}$

Mossstab : $\longmapsto-1 \cdot 10^{-3}$

$1---1+1 \cdot 10^{-3}$

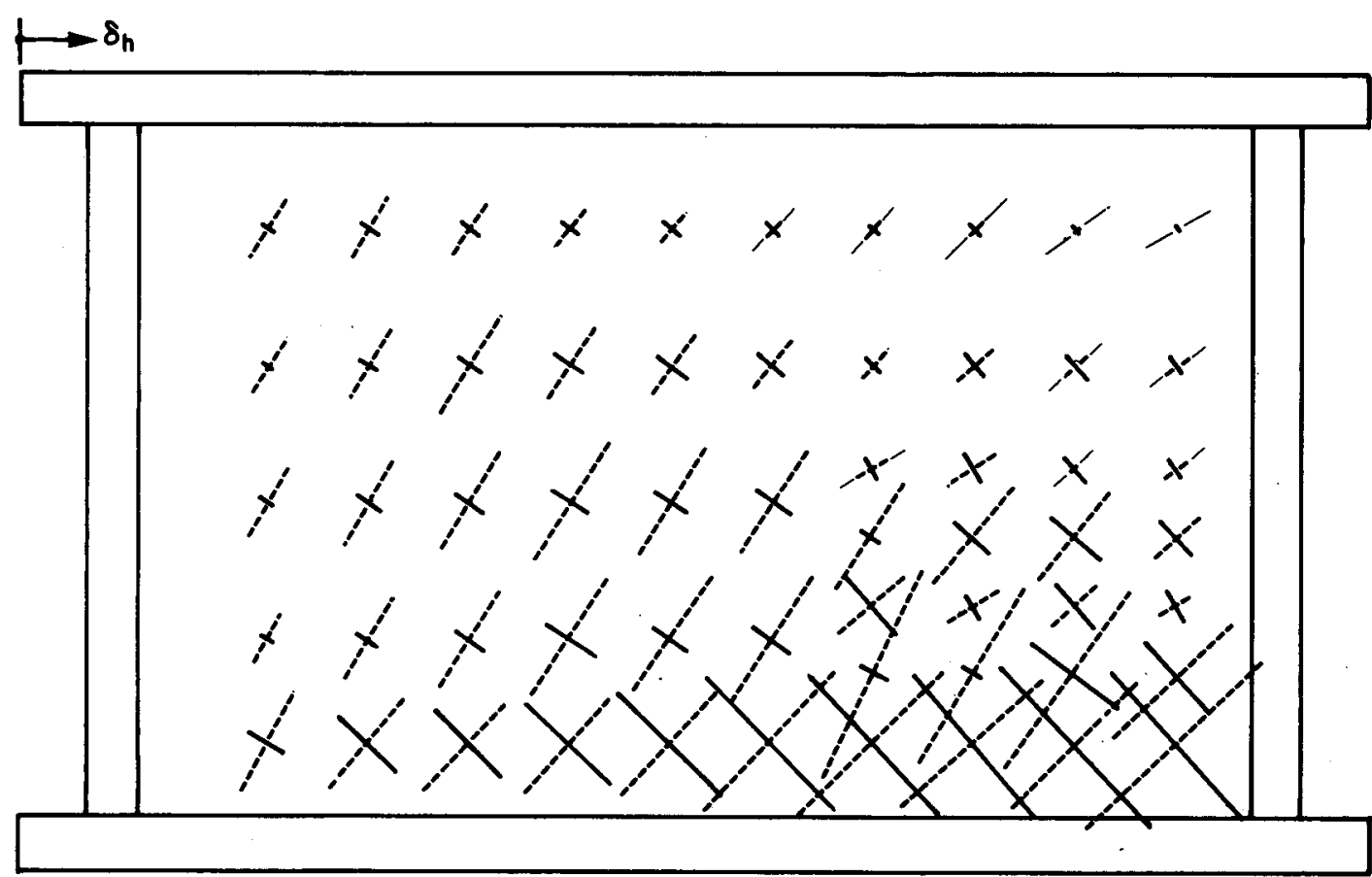

LS 14, $\quad \delta_{h}=24.14 \mathrm{~mm}$

Mossstab : $\longmapsto-10 \cdot 10^{-3}$

$1---1+10 \cdot 10^{-3}$

Bild 75: Houptdehnungen W 5 


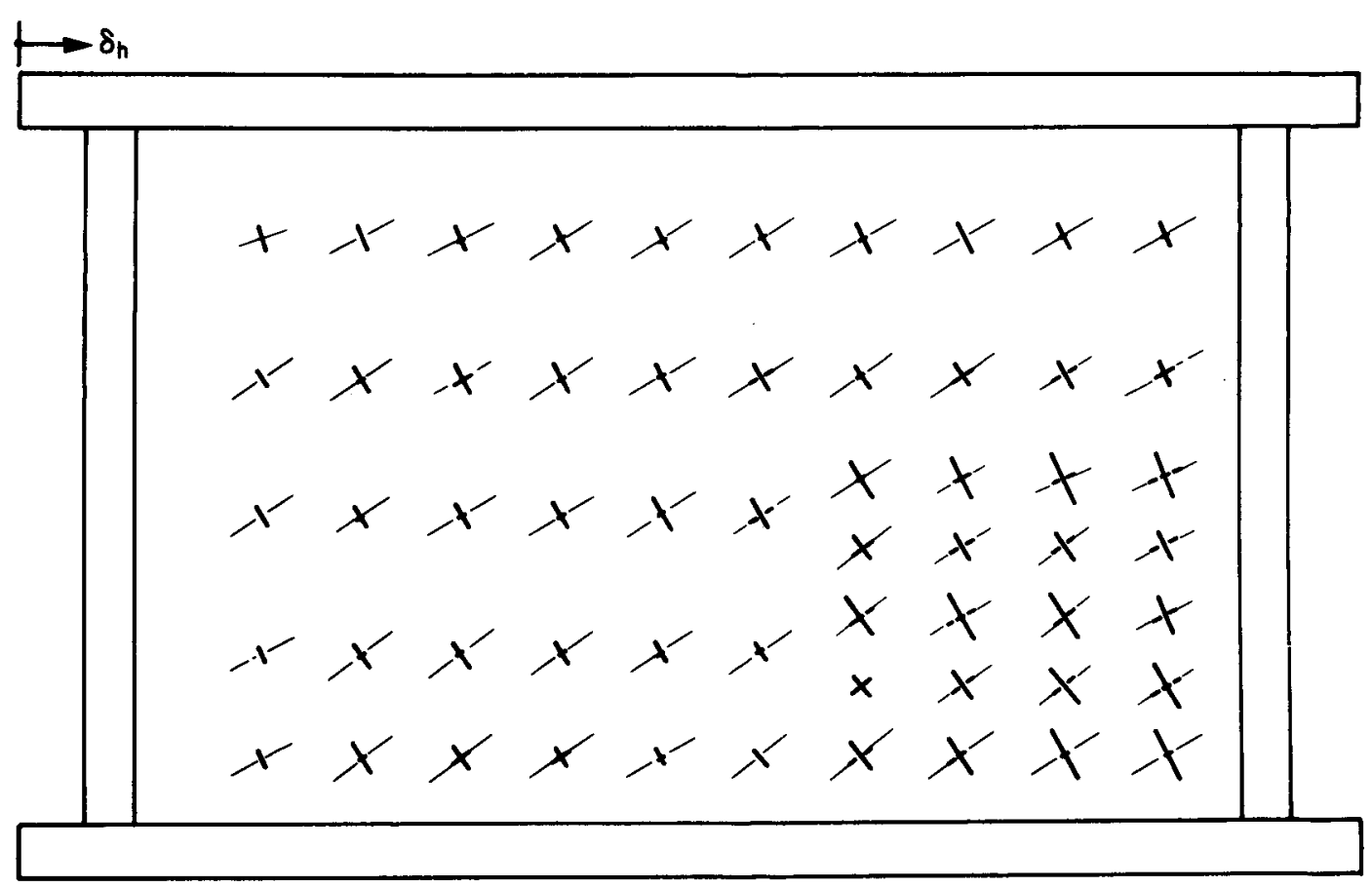

LS 5/1, $\quad \delta_{h}=+0.78 \mathrm{~mm}$

Mossstab : $\begin{array}{r}\longmapsto-1 \cdot 10^{-3} \\ \longmapsto---1+1 \cdot 10^{-3}\end{array}$

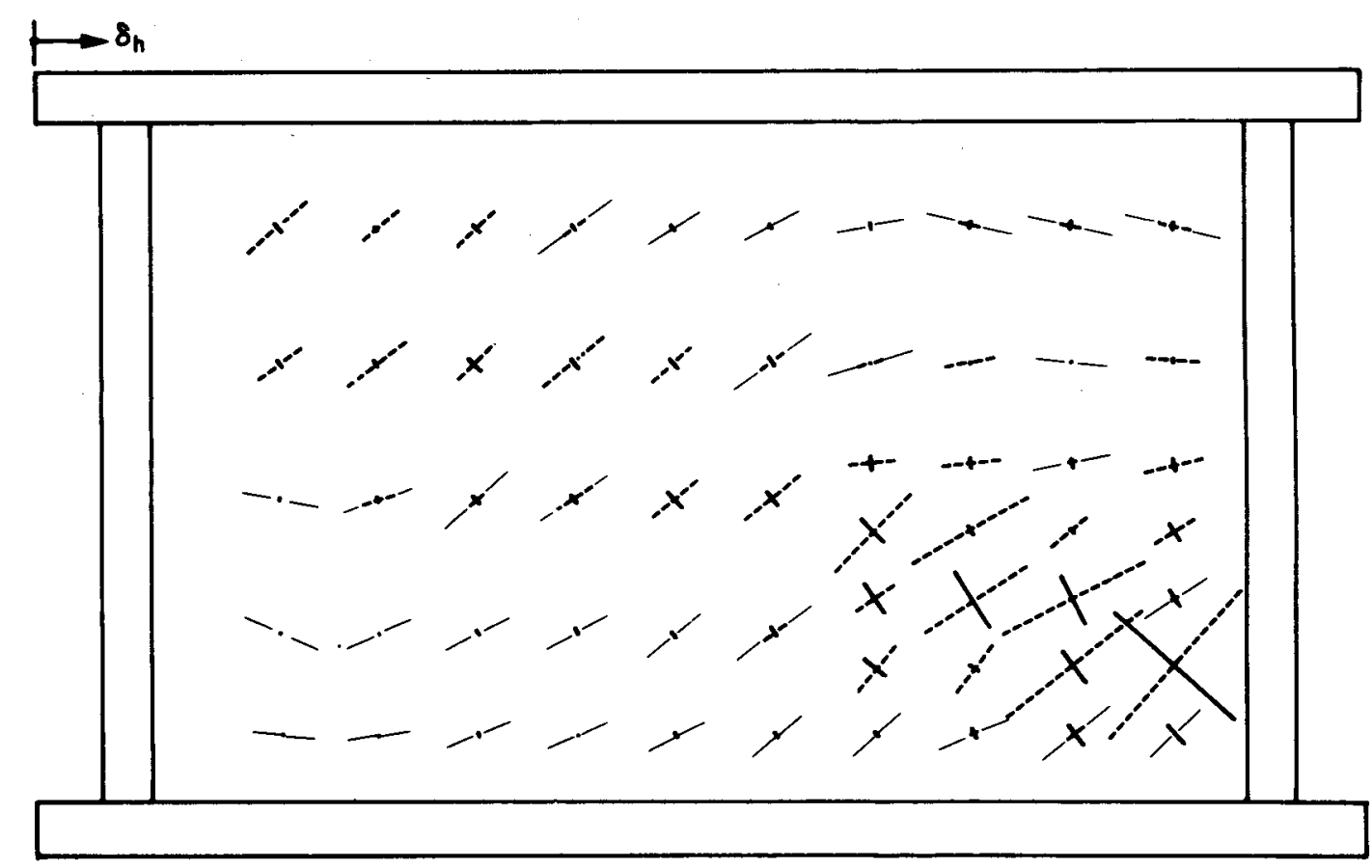

LS 8/2, $\quad \delta_{h}=+6.04 \mathrm{~mm}$

Massstab : $\begin{aligned} \longmapsto-10 \cdot 10^{-3} \\ \vdash--4+10 \cdot 10^{-3}\end{aligned}$

Bild 76: Hauptdehnungen W 6 


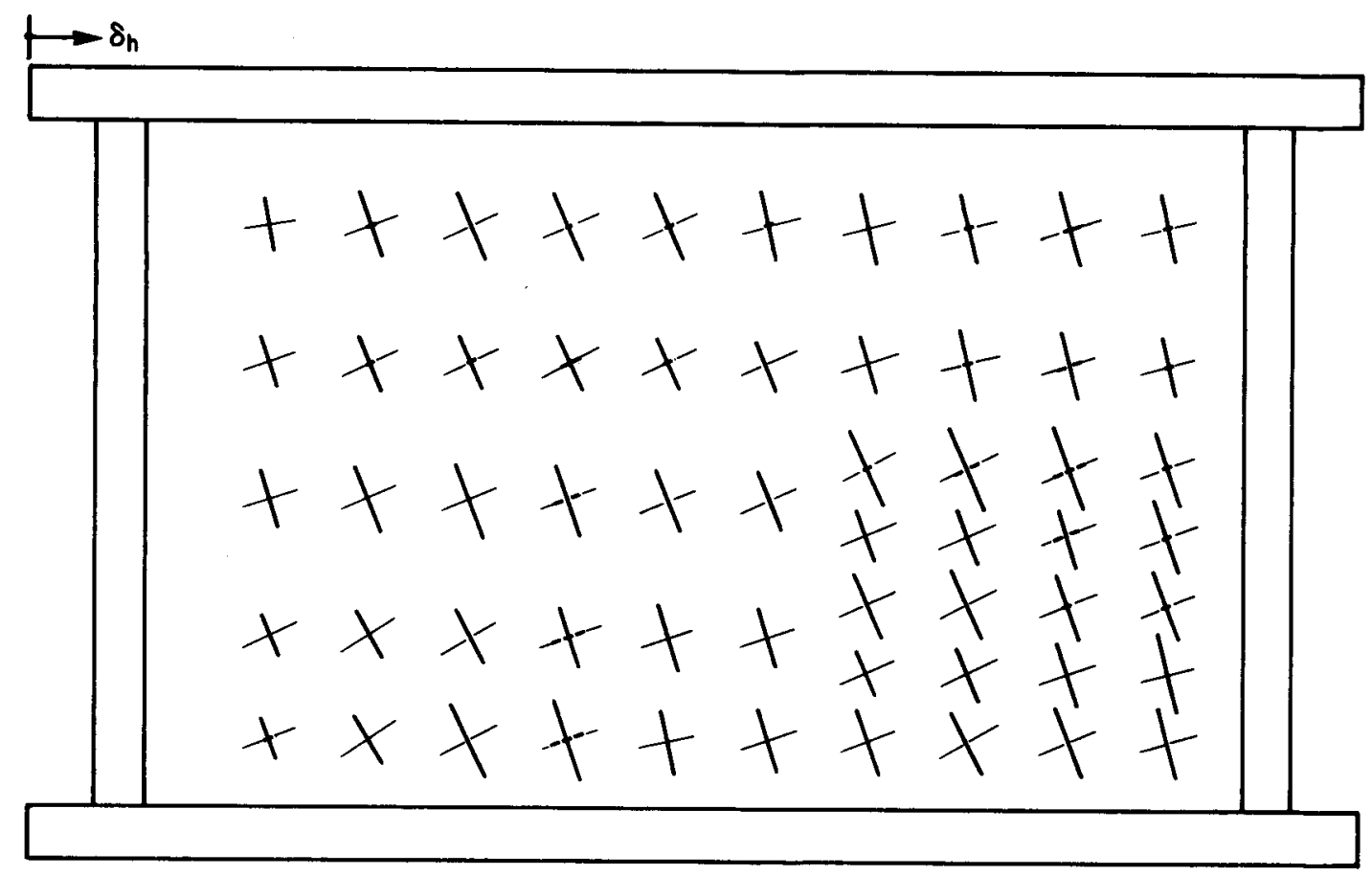
LS $5 / 1, \quad \delta_{h}=+0.78 \mathrm{~mm}$
Mossstob : $\longmapsto-1 \cdot 10^{-3}$
$1---1+1 \cdot 10^{-3}$

$$
\begin{aligned}
& \longmapsto \delta_{h}
\end{aligned}
$$

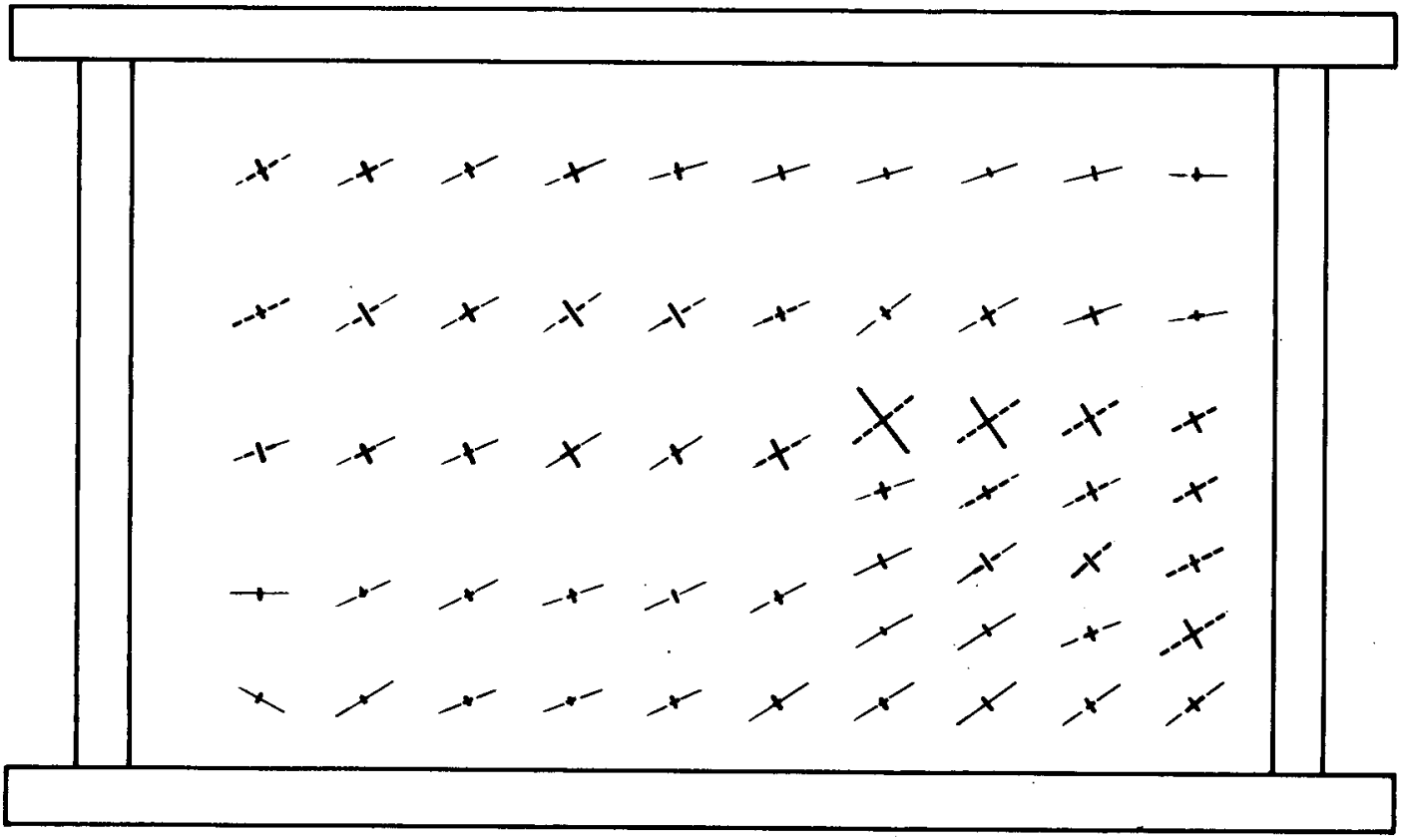

LS $9 / 2, \quad \delta_{h}=+3.96 \mathrm{~mm}$

Mossstob : $\longmapsto-10 \cdot 10^{-3}$

$1---1+10 \cdot 10^{-3}$

Bild 77: Hauptdehnungen $W 7$ 

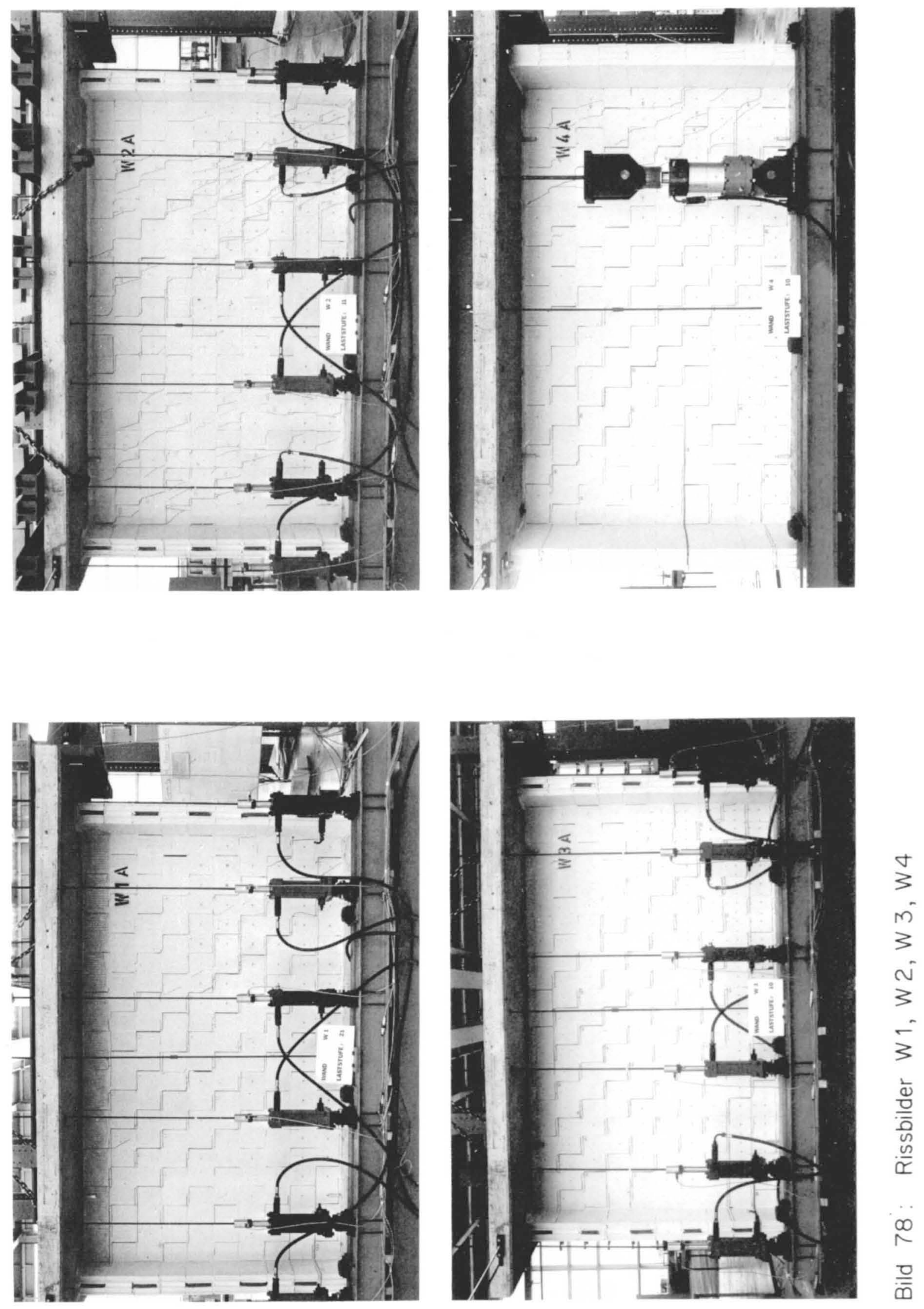

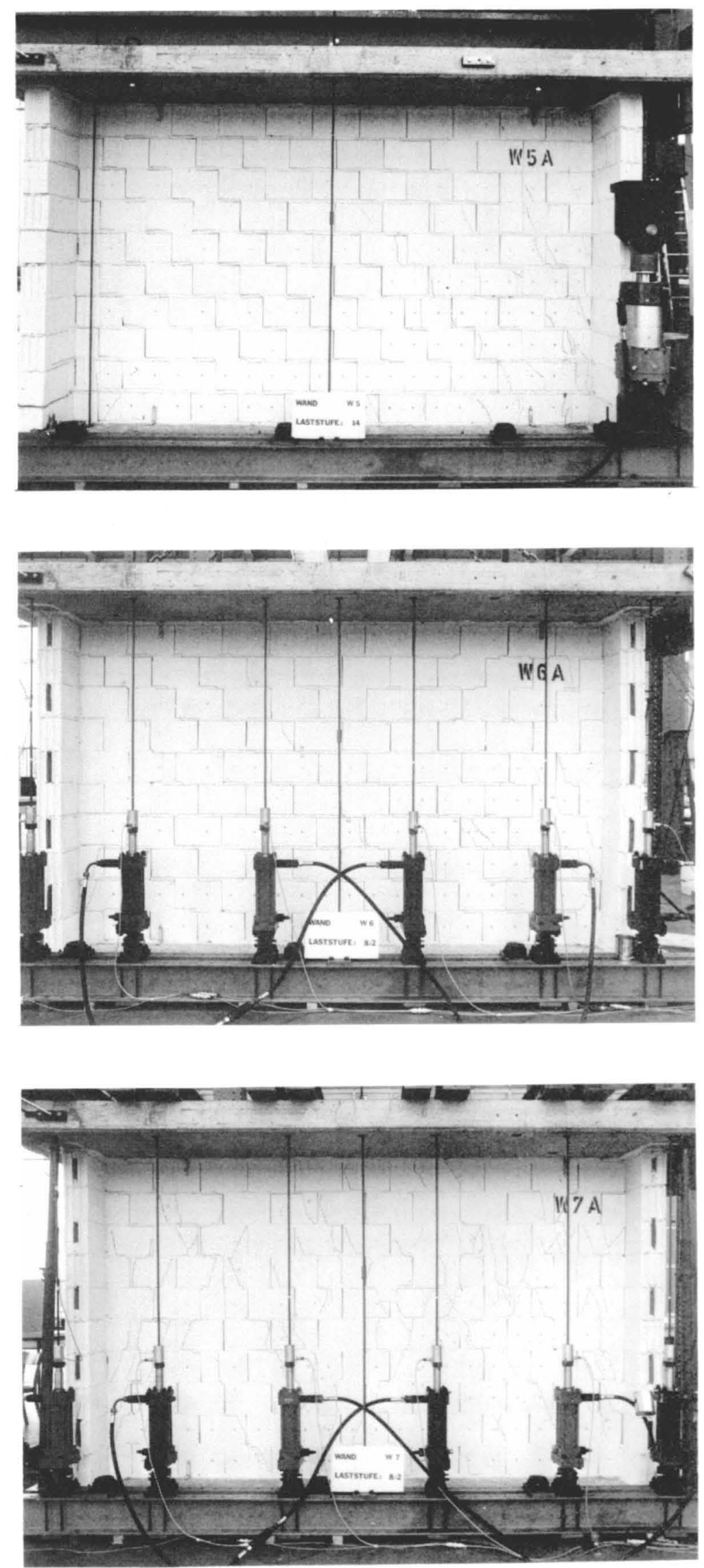

Bild 79: Rissbilder W 5, W6, W7 

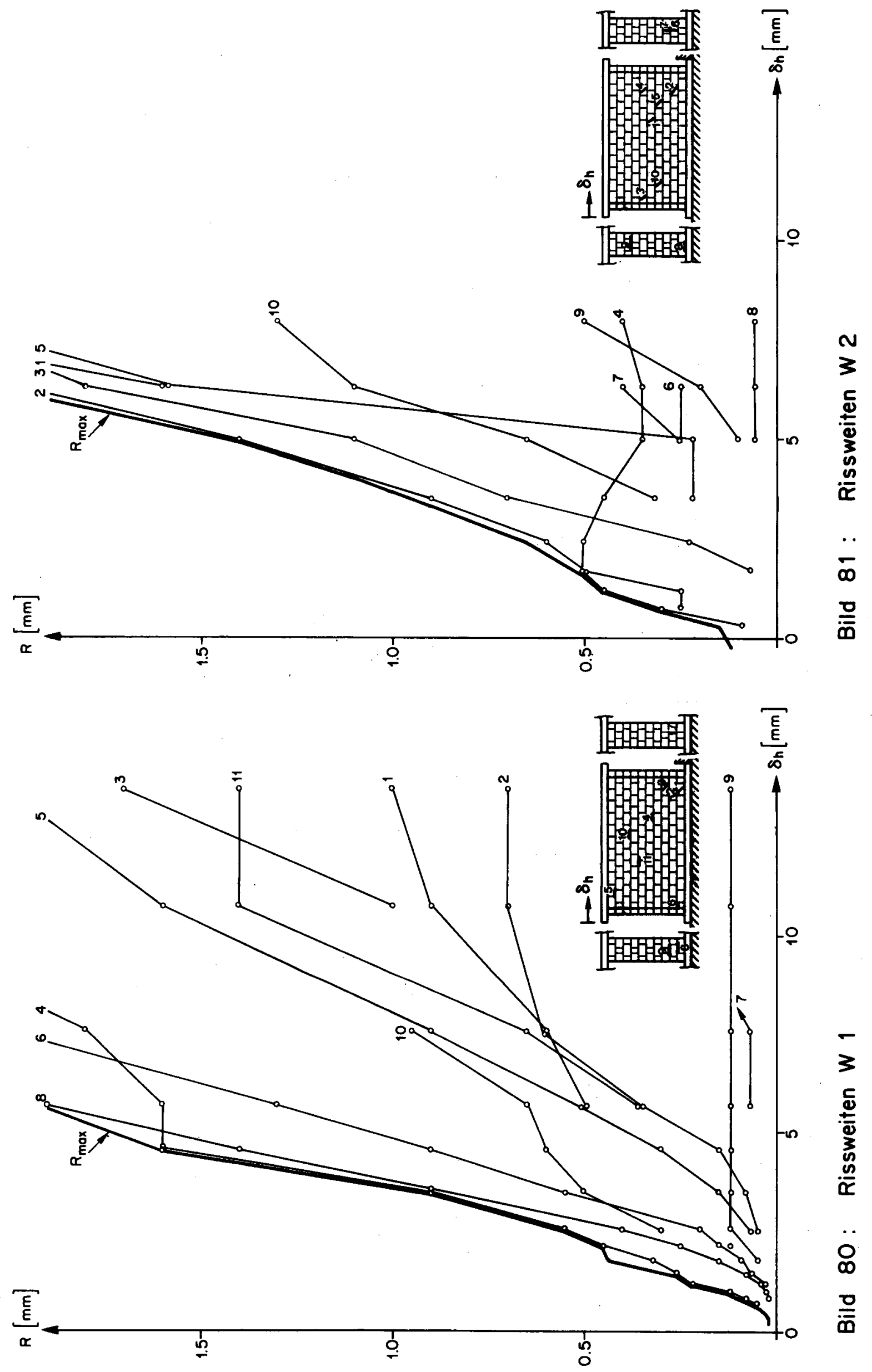

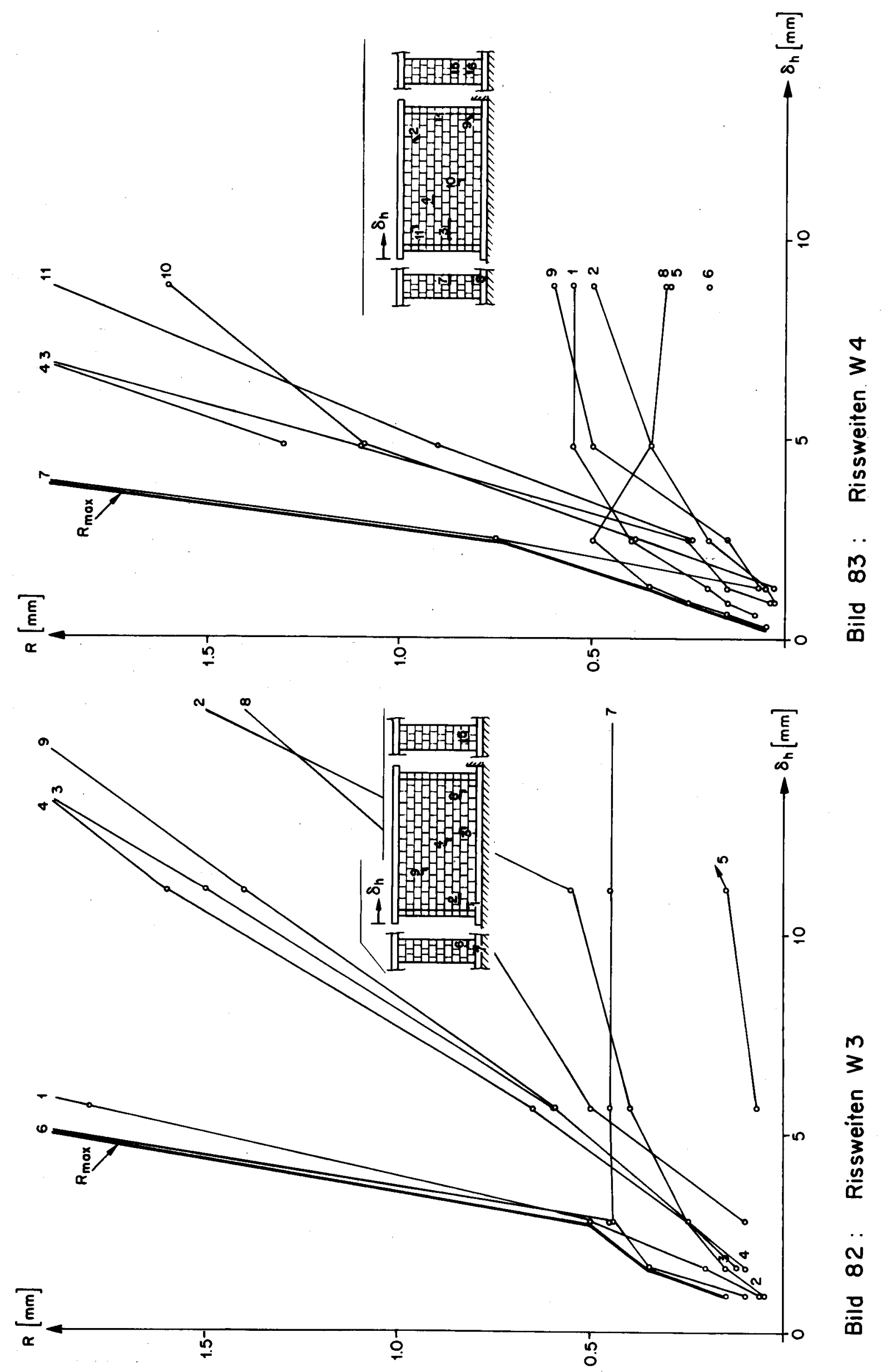


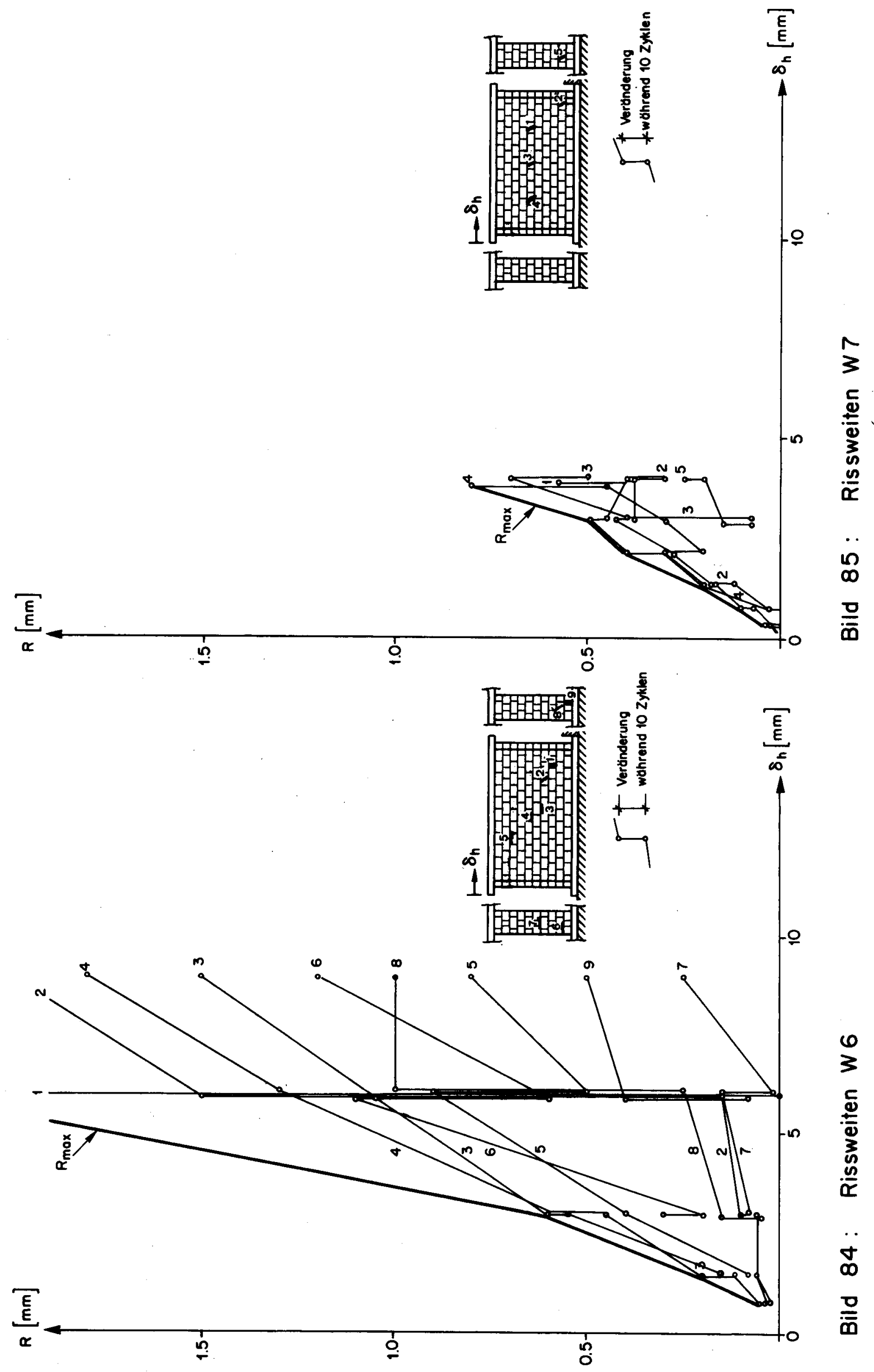




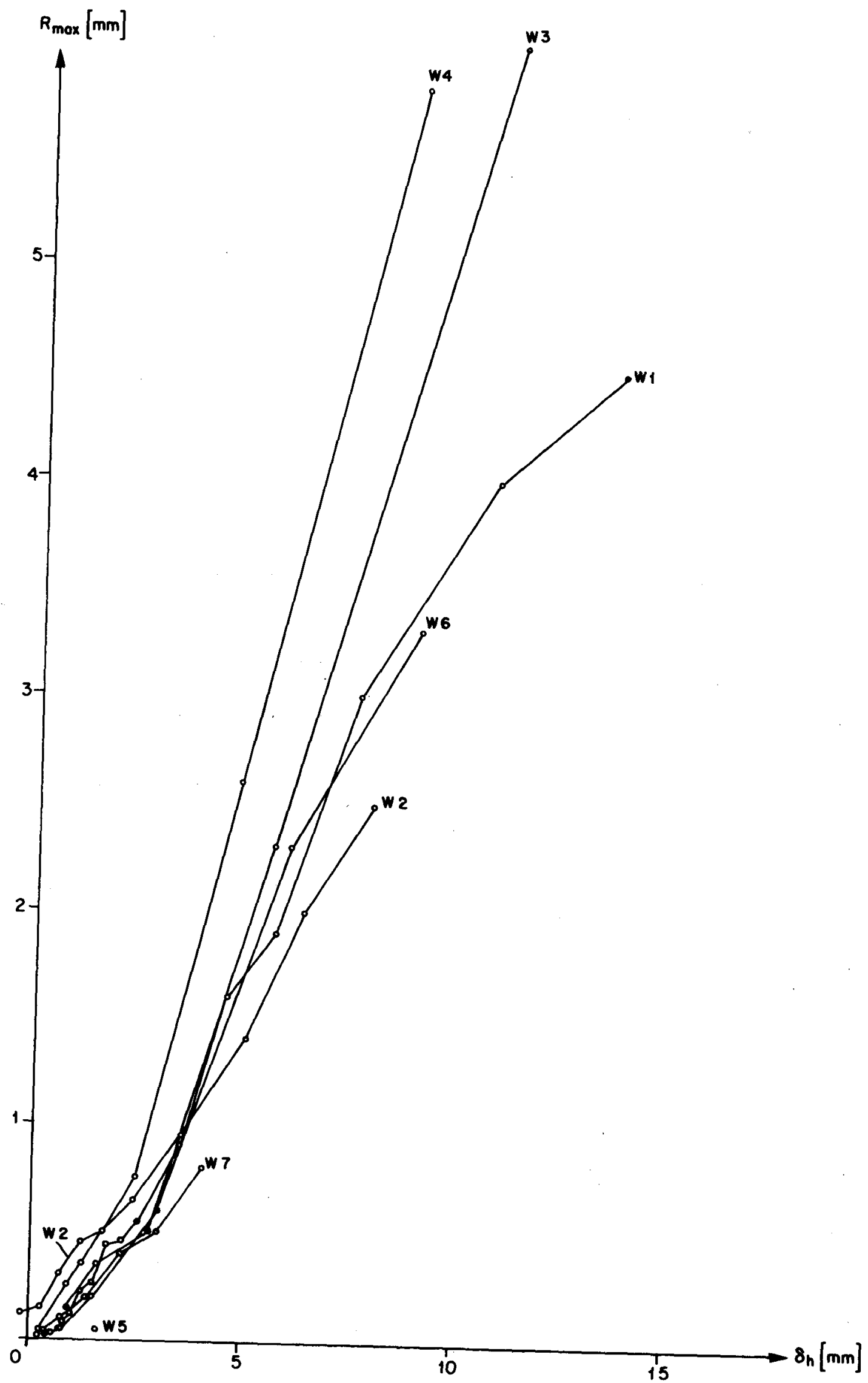

Bild 86: Max. Rissweiten 


\begin{tabular}{|c|c|c|c|c|c|c|c|}
\hline LS & $\begin{array}{c}V_{A} \\
{[k N]}\end{array}$ & $\begin{array}{l}V_{E} \\
{[k N]}\end{array}$ & $\frac{V_{A}-V_{E}}{V_{A}}$ & $\begin{array}{c}\delta_{h} \\
{[\mathrm{~mm}]}\end{array}$ & $\begin{array}{c}N_{E} \\
{[k N]}\end{array}$ & $\begin{array}{c}t_{A} \\
{[\text { Min] }}\end{array}$ & $\begin{array}{c}t_{E} \\
{[M i n]}\end{array}$ \\
\hline $1+2$ & 0.0 & 0.0 & - & 0.00 & 0.0 & - & 96 \\
\hline 3 & 0.0 & 0.0 & - & 0.04 & -416.0 & - & 80 \\
\hline 4 & 21.0 & 18.9 & 10 & 0.10 & -413.2 & 14 & 95 \\
\hline 5 & 48.0 & 45.7 & 5 & 0.21 & -413.5 & 7 & 89 \\
\hline 6 & 73.0 & 67.2 & 8 & 0.26 & -413.2 & 5 & 90 \\
\hline 7 & 91.5 & 88.9 & 3 & 0.37 & -413.4 & 6 & 82 \\
\hline 8 & 106.7 & 102.2 & 4 & 0.54 & -414.2 & 6 & 95 \\
\hline 9 & 127.0 & 121.5 & 4 & 0.67 & -414.7 & 8 & 98 \\
\hline 10 & 146.1 & 140.4 & 4 & 0.83 & -414.6 & 9 & 90 \\
\hline 11 & 165.1 & 158.7 & 4 & 1.00 & -414.9 & 8 & 90 \\
\hline 12 & 177.4 & 172.0 & 3 & 1.20 & -415.4 & 10 & 89 \\
\hline 13 & 194.7 & 188.4 & 3 & 1.47 & -414.7 & 9 & 98 \\
\hline 14 & 210.0 & 203.8 & 3 & 1.78 & -416.0 & 6 & 110 \\
\hline 15 & 223.0 & 216.5 & 3 & 2.16 & -416.0 & 7 & 111 \\
\hline 16 & 229.6 & 223.0 & 3 & 2.54 & -414.1 & 9 & 141 \\
\hline 17 & - & - & - & - & - & - & - \\
\hline 18 & 244.0 & 237.6 & 3 & 3.50 & -413.9 & 9 & 103 \\
\hline 19 & 254.4 & 247.8 & 3 & 4.55 & -413.1 & 10 & 94 \\
\hline 20 & 258.0 & 252.5 & 2 & 5.68 & -416.0 & 7 & 95 \\
\hline 21 & 266.2 & 260.4 & 2 & 7.59 & -416.3 & 18 & 103 \\
\hline 22 & 247.7 & 244.4 & 1 & 10.75 & -416.7 & 24 & 87 \\
\hline 23 & 241.7 & 236.2 & 2 & 13.68 & -415.5 & 21 & 98 \\
\hline $24 / 1$ * & 231.9 & - & - & 15.33 & -414.9 & - & - \\
\hline $24 / 2^{\star}$ & 227.5 & - & - & 16.12 & -415.3 & - & - \\
\hline $24 / 3^{*}$ & 223.4 & - & - & 17.11 & -416.4 & - & - \\
\hline $24 / 4^{*}$ & 211.4 & - & - & 18.88 & -415.5 & - & - \\
\hline
\end{tabular}

* Zwischenlaststufe

Tabelle A1: Belastungsablauf W1

\begin{tabular}{|c|c|c|c|c|c|c|c|}
\hline$L S$ & $\begin{array}{c}V_{A} \\
{[k N]}\end{array}$ & $\begin{array}{c}V_{E} \\
{[k N]}\end{array}$ & $\begin{array}{c}\frac{V_{A}-V_{E}}{V_{A}} \\
{[\%]}\end{array}$ & $\begin{array}{c}\delta_{h} \\
{[\mathrm{~mm}]}\end{array}$ & $\begin{array}{c}\mathrm{N}_{E} \\
{[\mathrm{kN}]}\end{array}$ & $\begin{array}{c}t_{A} \\
{[M i n]}\end{array}$ & $\begin{array}{c}t_{E} \\
{[M i n]}\end{array}$ \\
\hline $1+2$ & 0.0 & 0.0 & - & 0.00 & 0.0 & - & 59 \\
3 & 0.0 & 0.0 & - & -0.22 & -1284.5 & 118 & 75 \\
4 & 120.4 & 108.6 & 10 & 0.30 & -1288.1 & 12 & 84 \\
5 & 195.6 & 178.6 & 9 & 0.72 & -1286.9 & 7 & 80 \\
6 & 261.0 & 244.1 & 6 & 1.17 & -1284.1 & 7 & 78 \\
7 & 319.0 & 300.8 & 6 & 1.70 & -1284.7 & 8 & 65 \\
8 & 376.2 & 350.8 & 7 & 2.42 & -1285.6 & 10 & 122 \\
9 & 437.6 & 412.1 & 6 & 3.53 & -1283.9 & 9 & 101 \\
10 & 475.8 & 451.2 & 5 & 5.00 & -1287.5 & 15 & 109 \\
11 & 479.4 & 453.8 & 5 & 6.33 & -1284.9 & 13 & 111 \\
12 & 435.1 & 394.1 & 9 & 7.94 & -1285.6 & 13 & 95 \\
\hline
\end{tabular}

Tabelle A2: Belastungsablauf W2 


\begin{tabular}{|c|c|r|r|r|r|r|r|}
\hline$L S$ & \multicolumn{1}{|c|}{$\begin{array}{c}V_{A} \\
{[\mathrm{kN}]}\end{array}$} & $\begin{array}{c}V_{E} \\
{[\mathrm{kN}]}\end{array}$ & $\begin{array}{c}V_{A}-V_{E} \\
V_{A} \\
{[\%]}\end{array}$ & $\begin{array}{c}\delta_{h} \\
{[\mathrm{~mm}]}\end{array}$ & $\begin{array}{c}\mathrm{N}_{E} \\
{[\mathrm{kN}]}\end{array}$ & $\begin{array}{c}t_{A} \\
{[\text { Min] }]}\end{array}$ & $\begin{array}{c}t_{E} \\
{[\text { Min] }}\end{array}$ \\
\hline $1+2$ & 0.0 & 0.0 & - & 0.00 & 0.0 & - & 70 \\
3 & 0.0 & 0.0 & - & -0.08 & -412.0 & 81 & 84 \\
4 & 114.3 & 105.7 & 8 & 0.27 & -413.0 & 9 & 58 \\
5 & 195.2 & 186.5 & 4 & 0.93 & -413.3 & 24 & 65 \\
6 & 234.3 & 225.5 & 4 & 1.62 & -413.4 & 21 & 78 \\
7 & 253.7 & 244.4 & 4 & 2.81 & -412.6 & 26 & 93 \\
8 & 269.5 & 257.0 & 5 & 5.64 & -416.1 & 18 & 94 \\
9 & 273.6 & 264.4 & 3 & 11.15 & -413.1 & 26 & 75 \\
10 & 277.0 & 269.6 & 3 & 18.88 & -413.8 & 45 & 83 \\
11 & 275.4 & 273.1 & 1 & 31.03 & -414.1 & 58 & 42 \\
$12 / 4^{*}$ & 271.9 & - & - & 42.74 & -415.7 & 37 & - \\
$12 / 6^{*}$ & 268.4 & - & - & 50.74 & -414.7 & 16 & - \\
$12 / 8^{*}$ & 254.1 & - & - & 58.74 & -411.3 & 22 & - \\
$12 / 9^{*}$ & 180.5 & - & - & 62.74 & -411.3 & - & - \\
\hline
\end{tabular}

* Zwischenlaststufe

Tabelle A3: Belastungsablauf W3

\begin{tabular}{|c|c|c|c|c|c|c|c|}
\hline LS & $\begin{array}{c}V_{A} \\
{[k N]}\end{array}$ & $\begin{array}{c}V_{E} \\
{[k N]}\end{array}$ & $\frac{V_{A}-V_{E}}{V_{A}}$ & $\begin{array}{r}\delta_{h} \\
{[\mathrm{~mm}]}\end{array}$ & $\begin{array}{c}N_{E} \\
{[k N]}\end{array}$ & $\begin{array}{c}t_{A} \\
{[\operatorname{Min}]}\end{array}$ & $\begin{array}{c}t_{E} \\
{[\operatorname{Min}]}\end{array}$ \\
\hline $1+2$ & 0.0 & 0.0 & - & 0.00 & 0.0 & - & 72 \\
\hline 3 & 0.0 & 0.0 & - & 0.06 & -417.8 & 38 & 66 \\
\hline 4 & 50.7 & 48.1 & 5 & 0.29 & -417.6 & 5 & 60 \\
\hline 5 & 97.1 & 93.9 & 3 & 0.58 & -417.6 & 17 & 67 \\
\hline 6 & 122.6 & 117.7 & 4 & 0.87 & -416.9 & 8 & 64 \\
\hline 7 & 142.7 & 138.0 & 3 & 1.26 & -417.1 & 10 & 66 \\
\hline 8 & 169.4 & 165.0 & 3 & 2.45 & -420.3 & 24 & 77 \\
\hline 9 & 186.3 & 183.0 & 2 & 4.82 & -422.4 & 34 & 98 \\
\hline 10 & 189.8 & 186.7 & 2 & 8.84 & -421.6 & 45 & 81 \\
\hline 11 & 181.8 & 179.9 & 1 & 17.86 & -423.0 & 41 & 12 \\
\hline $12 / 1^{*}$ & 176.8 & - & - & 19.86 & -424.4 & 5 & - \\
\hline $12 / 2^{\star}$ & 171.0 & - & - & 21.87 & -422.9 & 3 & - \\
\hline $12 / 3^{*}$ & 162.5 & - & - & 22.22 & -419.8 & 6 & - \\
\hline
\end{tabular}

* Zwischenlaststufe

Tabelle A4: Belastungsablauf W4 


\begin{tabular}{|c|c|c|c|c|c|c|c|}
\hline LS & $\begin{array}{c}V_{A} \\
{[k N]}\end{array}$ & $\begin{array}{c}V_{E} \\
{[k N]}\end{array}$ & $\frac{V_{A}-V_{E}}{V_{A}}$ & $\begin{array}{c}\delta_{h} \\
{[\mathrm{~mm}]}\end{array}$ & $\begin{array}{c}\mathrm{N}_{\mathrm{E}} \\
{[\mathrm{kN}]}\end{array}$ & $\begin{array}{c}t_{A} \\
{[\operatorname{Min}]}\end{array}$ & $\begin{array}{c}t_{E} \\
{[\operatorname{Min}]}\end{array}$ \\
\hline $1,2,3$ & 0.0 & 0.0 & - & 0.00 & 0.0 & - & 50 \\
\hline 4 & 0.0 & 0.0 & - & 0.29 & -422.2 & 34 & 48 \\
\hline 5 & 88.6 & 82.0 & 7 & 0.85 & -422.8 & 13 & 54 \\
\hline 6 & 168.5 & 162.3 & 4 & 1.57 & -422.5 & 15 & 94 \\
\hline 8 & 0.0 & 0.0 & - & 0.00 & 0.0 & - & 81 \\
\hline 9 & 0.0 & 0.0 & - & 0.81 & -422.8 & 13 & 9 \\
\hline 10 & 163.4 & 159.7 & 2 & 4.14 & -422.2 & 18 & 22 \\
\hline 11 & 250.4 & 246.8 & 1 & 8.11 & -423.1 & 24 & 21 \\
\hline 12 & 316.6 & 311.8 & 2 & 12.14 & -425.6 & 25 & 21 \\
\hline 13 & 378.3 & 360.7 & 2 & 18.11 & -428.3 & 18 & 17 \\
\hline 14 & 362.0 & 340.9 & 6 & 24.14 & -426.5 & 9 & 93 \\
\hline $15 / 1^{*}$ & 357.2 & - & - & 26.18 & -423.5 & 4 & - \\
\hline $15 / 2^{*}$ & 327.9 & - & - & 28.20 & -420.0 & 4 & - \\
\hline
\end{tabular}

* Zwischenlaststufe

Tabelle A5: Belastungsablauf W5

\begin{tabular}{|c|c|c|c|r|r|r|r|}
\hline$L S$ & $\begin{array}{c}V_{A} \\
{[\mathrm{kN}]}\end{array}$ & $\begin{array}{c}V_{E} \\
{[\mathrm{kN}]}\end{array}$ & $\begin{array}{c}V_{A}-V_{E} \\
V_{A} \\
{[\%]}\end{array}$ & $\begin{array}{c}\delta_{h} \\
{[\mathrm{~mm}]}\end{array}$ & $\begin{array}{c}\mathrm{N}_{E} \\
{[\mathrm{kN}]}\end{array}$ & $\begin{array}{c}T_{10} \\
{[M i n]}\end{array}$ & $\begin{array}{c}t_{E} \\
{[M i n]}\end{array}$ \\
\hline $1+2$ & 0.0 & 0.0 & - & 0.00 & 0.0 & - & 60 \\
3 & 0.0 & 0.0 & - & 0.01 & -413.9 & - & 89 \\
$4 / 1$ & 75.0 & 68.5 & 9 & 0.30 & -417.8 & 79 & 75 \\
$4 / 2$ & 77.0 & 72.8 & 5 & 0.30 & -419.3 & & 62 \\
$5 / 1$ & 167.8 & 160.2 & 5 & 0.78 & -419.4 & 72 & 62 \\
$5 / 2$ & 163.0 & 157.8 & 3 & 0.78 & -420.0 & & 74 \\
$6 / 1$ & 220.4 & 213.6 & 3 & 1.49 & -418.5 & 75 & 72 \\
$6 / 2$ & 208.3 & 205.1 & 2 & 1.49 & -419.7 & & 62 \\
$7 / 1$ & 246.0 & 239.7 & 3 & 2.98 & -418.5 & 76 & 65 \\
$7 / 2$ & 234.9 & 231.1 & 2 & 2.98 & -418.7 & & 73 \\
$8 / 1$ & 253.3 & 247.3 & 2 & 6.03 & -413.6 & 110 & 82 \\
$8 / 2$ & 224.9 & 222.7 & 1 & 6.04 & -414.2 & & 84 \\
$9 / 1$ & 234.6 & 229.9 & 2 & 9.04 & -415.4 & $T_{7}=90$ & 60 \\
$9 / 2 *$ & 148.9 & - & - & 9.05 & -414.7 & & - \\
\hline
\end{tabular}

Tabe1le A6: Statischer Belastungsablauf W6

* Zwischenlaststufe 


\begin{tabular}{|c|r|r|r|r|r|r|r|}
\hline$L S$ & \multicolumn{1}{|c|}{$\begin{array}{c}V_{A} \\
{[\mathrm{kN}]}\end{array}$} & $\begin{array}{c}V_{E} \\
{[\mathrm{kN}]}\end{array}$ & $\begin{array}{c}V_{A}-V_{E} \\
V_{A} \\
{[\%]}\end{array}$ & $\begin{array}{c}\delta_{h} \\
{[\mathrm{~mm}]}\end{array}$ & $\begin{array}{c}N_{E} \\
{[\mathrm{kN}]}\end{array}$ & $\begin{array}{c}T_{10} \\
{[M i n]}\end{array}$ & $\begin{array}{c}t_{E} \\
{[M i n]}\end{array}$ \\
\hline $1+2$ & 0.0 & 0.0 & - & 0.00 & 0.0 & - & 56 \\
3 & 0.0 & 0.0 & - & -0.08 & -1289.2 & - & 55 \\
$4 / 1$ & 128.9 & 120.4 & 7 & 0.39 & -1283.7 & 76 & 58 \\
$4 / 2$ & 133.9 & 129.1 & 4 & 0.39 & -1292.4 & & 54 \\
$5 / 1$ & 243.7 & 231.9 & 5 & 0.78 & -1290.1 & 80 & 58 \\
$5 / 2$ & 240.6 & 234.3 & 3 & 0.77 & -1289.2 & & 65 \\
$6 / 1$ & 356.2 & 338.9 & 5 & 1.37 & -1288.8 & 79 & 67 \\
$6 / 2$ & 337.7 & 328.7 & 3 & 1.37 & -1289.6 & & 53 \\
$7 / 1$ & 447.8 & 431.2 & 4 & 2.16 & -1287.5 & 89 & 66 \\
$7 / 2$ & 417.9 & 406.9 & 3 & 2.16 & -1289.4 & & 63 \\
$8 / 1$ & 504.4 & 488.9 & 3 & 2.98 & -1286.8 & 103 & 64 \\
$8 / 2$ & 448.5 & 437.5 & 2 & 2.98 & -1289.8 & & 70 \\
$9 / 1$ & 510.9 & 491.3 & 4 & 3.96 & -1288.4 & 83 & 71 \\
$9 / 2$ & 383.4 & 371.5 & 3 & 3.96 & -1290.1 & & 88 \\
\hline
\end{tabular}

Tabelle A7: Statischer Belastungsablauf W7 


\begin{tabular}{|c|c|c|c|c|c|c|c|c|c|c|c|}
\hline \multicolumn{2}{|c|}{ LS 4} & \multicolumn{2}{|c|}{ LS 5} & \multicolumn{2}{|c|}{ LS 6} & \multicolumn{2}{|c|}{ LS 7} & \multicolumn{2}{|c|}{ LS 8} & \multicolumn{2}{|c|}{ LS 9} \\
\hline $\begin{array}{c}V_{A} \\
{[k N]}\end{array}$ & $\stackrel{\delta_{h}}{[\mathrm{~mm}]}$ & $\begin{array}{c}V_{A} \\
{[k N]}\end{array}$ & $\stackrel{\delta_{h}}{[\mathrm{~mm}]}$ & $\begin{array}{c}V_{A} \\
{[k N]}\end{array}$ & $\stackrel{\delta_{h}}{[\mathrm{~mm}]}$ & $\begin{array}{c}V_{A} \\
{[k N]}\end{array}$ & $\begin{array}{c}\delta_{h} \\
{[\mathrm{~mm}]}\end{array}$ & $\begin{array}{c}V_{A} \\
{[k N]}\end{array}$ & $\begin{array}{c}\delta_{h} \\
{[\mathrm{~mm}]}\end{array}$ & $\begin{array}{c}V_{A} \\
{[k N]}\end{array}$ & $\begin{array}{c}\delta_{\mathrm{h}} \\
{[\mathrm{mm}]}\end{array}$ \\
\hline 0.0 & 0.00 & 3.8 & 0.00 & 0.9 & 0.09 & 13.4 & 0.01 & 1.1 & 0.22 & 68.7 & 0.05 \\
\hline 51.3 & 0.20 & 99.2 & 0.39 & 149.1 & 0.75 & 160.2 & 1.00 & 201.8 & 2.03 & 183.7 & 3.04 \\
\hline 76.1 & 0.30 & 170.2 & 0.79 & 220.4 & 1.49 & 234.1 & 1.99 & 249.6 & 4.02 & 225.4 & 6.04 \\
\hline 75.0 & 0.30 & 167.8 & 0.78 & 213.6 & 1.49 & 247.7 & 2.98 & 254.5 & 6.02 & 235.9 & 9.04 \\
\hline 68.5 & 0.29 & 160.2 & 0.78 & 86.8 & 0.75 & 246.0 & 2.99 & 253.3 & 6.03 & 234.6 & 9.04 \\
\hline 41.7 & 0.20 & 58.3 & 0.38 & -59.4 & 0.00 & 239.7 & 2.98 & 247.3 & 6.03 & 229.9 & 9.04 \\
\hline-15.9 & 0.00 & -37.0 & -0.02 & -161.6 & -0.61 & 184.0 & 1.98 & 211.5 & 4.02 & 189.4 & 6.03 \\
\hline-65.6 & -0.19 & -123.6 & -0.41 & -224.3 & -1.35 & 73.1 & 0.99 & 106.1 & 2.02 & 37.1 & 3.03 \\
\hline-90.7 & -0.29 & -180.6 & -0.77 & -93.9 & -0.59 & -70.3 & 0.00 & -101.3 & 0.03 & -132.7 & 0.04 \\
\hline-60.2 & -0.19 & -88.8 & -0.41 & 16.6 & 0.01 & -184.7 & -0.99 & -212.3 & -1.96 & -210.2 & -2.97 \\
\hline-3.7 & -0.01 & 4.4 & -0.01 & 216.4 & 1.50 & -242.4 & -2.00 & -249.3 & -3.96 & -242.6 & -5.98 \\
\hline 74.6 & 0.30 & 165.5 & 0.78 & -219.8 & -1.35 & -248.5 & -3.00 & -256.6 & -5.95 & -254.9 & -8.98 \\
\hline-90.0 & -0.29 & -176.0 & -0.77 & 215.7 & 1.49 & -190.8 & -1.99 & -218.4 & -3.95 & -221.2 & -5.96 \\
\hline 75.0 & 0.30 & 165.7 & 0.78 & -217.3 & -1.35 & -86.3 & -0.98 & -128.2 & -1.94 & -125.6 & -2.97 \\
\hline-89.8 & -0.30 & -172.0 & -0.77 & 215.1 & 1.49 & 47.9 & 0.01 & 67.7 & 0.05 & 71.2 & 0.03 \\
\hline 74.5 & 0.30 & 165.3 & 0.78 & -216.2 & -1.35 & 241.1 & 2.99 & 248.9 & 6.04 & 225.6 & 9.04 \\
\hline-88.9 & -0.29 & -172.7 & -0.77 & 213.5 & 1.49 & -242.8 & -2.99 & -249.2 & -5.96 & -246.6 & -8.98 \\
\hline 74.5 & 0.29 & 165.9 & 0.78 & -215.4 & -1.35 & 239.8 & 2.99 & 240.3 & 6.05 & 219.5 & 9.05 \\
\hline-89.2 & -0.30 & -171.2 & -0.77 & 213.6 & 1.49 & -240.3 & -2.99 & -245.4 & -5.96 & -243.6 & -8.98 \\
\hline 75.3 & 0.30 & 164.7 & 0.77 & -214.3 & -1.35 & 238.7 & 2.99 & 235.3 & 6.04 & 210.1 & 9.05 \\
\hline-88.1 & -0.29 & -169.5 & -0.77 & 213.2 & 1.49 & -240.0 & -3.00 & -245.8 & -5.95 & -238.5 & -8.98 \\
\hline 75.3 & 0.30 & 164.9 & 0.78 & -212.6 & -1.35 & 237.8 & 2.99 & 237.4 & 6.04 & 197.5 & 9.05 \\
\hline-88.7 & -0.29 & -169.6 & -0.77 & 212.2 & 1.49 & -239.1 & -3.00 & -247.4 & -5.96 & -231.1 & -8.99 \\
\hline 75.0 & 0.30 & 164.1 & 0.78 & -213.1 & -1.35 & 237.8 & 2.99 & 236.0 & 6.05 & 188.8 & 9.05 \\
\hline-88.6 & -0.29 & -168.7 & -0.77 & 211.4 & 1.49 & -238.7 & -3.00 & -247.5 & -5.96 & -216.2 & -8.99 \\
\hline 74.7 & 0.30 & 163.9 & 0.78 & -212.3 & -1.35 & 237.0 & 2.99 & 234.0 & 6.04 & 175.8 & 9.05 \\
\hline-88.5 & -0.29 & -167.8 & -0.79 & 14.7 & 0.01 & -237.7 & -3.00 & -246.3 & -5.96 & -199.9 & -8.98 \\
\hline-5.6 & 0.00 & 3.0 & -0.02 & 128.6 & 0.75 & 236.5 & 2.99 & 233.3 & 6.05 & 148.9 & 9.05 \\
\hline 48.3 & 0.20 & 84.0 & 0.38 & 210.5 & 1.49 & -236.9 & -3.00 & -246.0 & -5.96 & -158.5 & -8.99 \\
\hline 75.7 & 0.30 & 163.6 & 0.78 & 99.1 & 0.74 & 236.5 & 2.99 & 231.4 & 6.04 & 0.0 & -4.44 \\
\hline 46.2 & 0.20 & 70.7 & 0.37 & -32.4 & 0.00 & -236.1 & -2.99 & -243.8 & -5.96 & & \\
\hline-10.7 & 0.00 & -17.1 & -0.02 & -123.2 & -0.59 & 56.3 & 0.00 & 67.4 & 0.04 & & \\
\hline-62.4 & -0.19 & -98.2 & -0.41 & -211.7 & -1.35 & 145.7 & 0.99 & 153.8 & 2.04 & & \\
\hline-87.9 & -0.30 & -167.4 & -0.77 & -91.0 & -0.59 & 205.2 & 1.99 & 208.0 & 4.04 & & \\
\hline-59.2 & -0.20 & -83.6 & -0.41 & 13.4 & 0.01 & 234.8 & 2.99 & 229.8 & 6.05 & & \\
\hline-3.8 & 0.00 & 4.6 & -0.02 & 160.2 & 1.00 & 187.6 & 1.99 & 185.5 & 4.04 & & \\
\hline 76.8 & 0.30 & 163.1 & 0.78 & 210.3 & 1.49 & 79.2 & 0.99 & 32.0 & 2.04 & & \\
\hline 77.0 & 0.30 & 163.0 & 0.79 & 208.3 & 1.49 & -48.2 & 0.00 & -105.2 & 0.04 & & \\
\hline 72.8 & 0.29 & $\begin{array}{r}157.8 \\
-\quad 1.1\end{array}$ & $\begin{array}{l}0.77 \\
0.06\end{array}$ & 205.1 & 1.49 & $\begin{array}{r}-143.6 \\
-206.6 \\
-236.4 \\
-185.2 \\
-\quad 65.2 \\
58.7 \\
235.5 \\
234.9 \\
231.1 \\
0.0\end{array}$ & $\begin{array}{r}-0.99 \\
-1.99 \\
-3.00 \\
-1.99 \\
-0.99 \\
0.00 \\
2.99 \\
2.99 \\
2.98 \\
0.40\end{array}$ & $\begin{array}{r}-179.8 \\
-224.7 \\
-244.3 \\
-210.9 \\
-90.0 \\
68.7 \\
183.7 \\
225.4 \\
224.9 \\
222.7\end{array}$ & $\begin{array}{r}-1.95 \\
-3.95 \\
-5.95 \\
-3.95 \\
-1.95 \\
0.05 \\
3.04 \\
6.04 \\
6.04 \\
6.04\end{array}$ & & \\
\hline
\end{tabular}

Tabelle A8: Zyklischer Belastungsablauf W6 


\begin{tabular}{|c|c|c|c|c|c|c|c|c|c|c|c|}
\hline \multicolumn{2}{|c|}{ LS 4} & \multicolumn{2}{|c|}{ LS 5} & \multicolumn{2}{|c|}{ LS 6} & \multicolumn{2}{|c|}{ LS 7} & \multicolumn{2}{|c|}{ LS 8} & \multicolumn{2}{|c|}{ LS 9} \\
\hline $\begin{array}{c}\mathrm{V}_{\mathrm{A}} \\
{[\mathrm{kN}]}\end{array}$ & $\underset{[\mathrm{m}]}{\delta_{\mathrm{m}}}$ & $\begin{array}{c}V_{A} \\
{[\mathrm{kN}]}\end{array}$ & $\begin{array}{c}\delta_{h} \\
{[\mathrm{~mm}]}\end{array}$ & $\begin{array}{c}\mathrm{V}_{\mathrm{A}} \\
{[\mathrm{kN}]}\end{array}$ & $\underset{[\mathrm{mm}]}{\delta_{\mathrm{h}}}$ & $\underset{[\mathrm{kN}]}{\mathrm{V}_{\mathrm{A}}}$ & $\begin{array}{c}\delta_{h} \\
{[\mathrm{~mm}]}\end{array}$ & $\begin{array}{c}V_{A} \\
{[\mathrm{kN}]}\end{array}$ & $\underset{[\mathrm{mm}]}{\delta_{h}}$ & $\begin{array}{l}v_{A} \\
{[k N]}\end{array}$ & $\begin{array}{c}\delta_{h} \\
{[\mathrm{~mm}]}\end{array}$ \\
\hline 0.0 & 0.00 & 2.4 & 0.00 & 2.0 & -0.02 & 23.9 & -0.02 & 1.9 & 0.15 & 45.4 & -0.01 \\
\hline 116.8 & 0.35 & 135.1 & 0.39 & 240.3 & 0.78 & 204.3 & 0.77 & 266.0 & 1.38 & 243.7 & 1.38 \\
\hline 129.8 & 0.39 & 246.4 & 0.79 & 361.2 & 1.37 & 339.1 & 1.37 & 406.6 & 2.18 & 450.8 & 2.98 \\
\hline 128.9 & 0.40 & 243.7 & 0.78 & 356.2 & 1.37 & 452.6 & 2.16 & 507.4 & 2.98 & 514.5 & 3.96 \\
\hline 120.4 & 0.39 & 231.9 & 0.78 & 338.9 & 1.37 & 447.8 & 2.16 & 504.4 & 2.98 & 510.9 & 3.96 \\
\hline 50.6 & 0.20 & 97.6 & 0.38 & 165.4 & 0.77 & 431.2 & 2.15 & 488.9 & 2.98 & 491.3 & 3.96 \\
\hline-17.1 & 0.00 & -26.2 & -0.01 & -45.5 & -0.02 & 246.4 & 1.36 & 344.7 & 2.18 & 347.3 & 2.96 \\
\hline - 79.7 & -0.20 & -146.6 & -0.41 & -252.7 & -0.81 & 117.9 & 0.76 & 199.3 & 1.38 & 123.5 & 1.37 \\
\hline-133.4 & -0.38 & -251.8 & -0.81 & -354.6 & -1.41 & -55.7 & -0.03 & -61.7 & -0.01 & -66.5 & -0.03 \\
\hline-65.8 & -0.20 & -113.5 & -0.41 & -176.7 & -0.81 & -218.4 & -0.82 & -282.9 & -1.40 & -235.1 & -1.42 \\
\hline 0.5 & 0.00 & 8.6 & -0.01 & 27.3 & -0.02 & -337.8 & -1.42 & -398.6 & -2.20 & -417.3 & -3.03 \\
\hline 132.6 & 0.39 & 242.6 & 0.78 & 355.4 & 1.37 & -429.5 & -2.23 & -469.8 & -3.00 & -481.3 & -4.02 \\
\hline-130.8 & -0.39 & -242.5 & -0.81 & -342.3 & -1.41 & -248.0 & -1.42 & -319.6 & -2.20 & -332.3 & -3.02 \\
\hline 134.4 & 0.39 & 244.0 & 0.78 & 352.2 & 1.37 & -123.8 & -0.82 & -187.8 & -1.40 & -121.2 & -1.42 \\
\hline-127.3 & -0.38 & -240.0 & -0.81 & -336.5 & -1.41 & 42.6 & -0.03 & 48.9 & 0.00 & 56.3 & -0.02 \\
\hline 134.4 & 0.39 & 242.4 & 0.77 & 349.1 & 1.37 & 442.2 & 2.16 & 486.1 & 2.98 & 487.2 & 3.96 \\
\hline-126.6 & -0.38 & -237.5 & -0.81 & -331.8 & -1.41 & -416.3 & -2.22 & -455.3 & -3.00 & -462.2 & -4.03 \\
\hline 134.6 & 0.39 & 244.0 & 0.78 & 346.1 & 1.37 & 437.6 & 2.16 & 478.7 & 2.98 & 474.8 & 3.96 \\
\hline-125.6 & -0.38 & -236.2 & -0.81 & -329.1 & -1.41 & -400.8 & -2.22 & -447.0 & -3.01 & -445.3 & -4.02 \\
\hline 134.6 & 0.39 & 243.9 & 0.78 & 345.6 & 1.37 & 434.6 & 2.16 & 473.4 & 2.98 & 460.8 & 3.96 \\
\hline-126.4 & -0.38 & -234.5 & -0.81 & -327.6 & -1.42 & -400.1 & -2.23 & -440.7 & -3.00 & -432.5 & -4.03 \\
\hline 133.9 & 0.39 & 242.1 & 0.78 & 342.3 & 1.37 & 430.8 & 2.16 & 469.6 & 2.98 & 447.6 & 3.96 \\
\hline-126.4 & -0.38 & -234.1 & -0.81 & -325.0 & -1.42 & -396.5 & -2.23 & -433.8 & -3.01 & -419.6 & -4.03 \\
\hline 134.6 & 0.39 & 241.8 & 0.78 & 344.0 & 1.37 & 427.8 & 2.16 & 463.8 & 2.98 & 436.7 & 3.97 \\
\hline-125.6 & -0.38 & -233.0 & -0.81 & -321.5 & -1.41 & -393.1 & -2.23 & -427.3 & -3.00 & -407.9 & -4.02 \\
\hline 134.4 & 0.39 & 241.8 & 0.77 & 341.7 & 1.37 & 426.2 & 2.15 & 462.0 & 2.98 & 426.3 & 3.97 \\
\hline-125.4 & -0.38 & -231.5 & -0.81 & $\mid-320.5$ & -1.42 & -390.7 & -2.23 & -423.7 & -3.01 & -398.0 & -4.03 \\
\hline 2.1 & 0.00 & 12.2 & -0.02 & 22.4 & -0.02 & 425.8 & 2.16 & 457.7 & 2.98 & 415.8 & 3.97 \\
\hline 68.6 & 0.19 & 125.6 & 0.38 & 203.1 & 0.77 & -386.8 & -2.22 & -418.4 & -3.00 & -384.8 & -4.03 \\
\hline 134.5 & 0.39 & 241.6 & 0.77 & 339.0 & 1.37 & 422.3 & 2.16 & 454.6 & 2.98 & 404.7 & 3.97 \\
\hline 66.3 & 0.19 & 115.2 & 0.37 & 183.0 & 0.77 & -385.7 & -2.23 & -416.5 & -3.00 & -374.6 & -4.03 \\
\hline-0.8 & 0.00 & -1.2 & -0.02 & - $\quad 6.7$ & -0.02 & 35.5 & -0.03 & 43.1 & -0.01 & 46.1 & -0.03 \\
\hline-65.0 & -0.20 & -114.0 & -0.41 & $\mid-186.1$ & -0.81 & 179.6 & 0.76 & 244.2 & 1.38 & 172.1 & 1.37 \\
\hline-.24 .0 & -0.38 & -232.1 & -0.81 & $\mid-319.6$ & -1.41 & 285.1 & 1.36 & 351.1 & 2.17 & 309.9 & 2.96 \\
\hline-62.4 & -0.20 & -104.6 & -0.41 & $\mid-165.7$ & -0.82 & 420.7 & 2.16 & 452.8 & 2.98 & 396.0 & 3.97 \\
\hline 2.4 & 0.00 & 12.7 & -0.02 & 23.9 & -0.02 & 256.4 & 1.36 & 328.7 & 2.18 & 261.3 & 2.96 \\
\hline 135.1 & 0.39 & 241.3 & 0.77 & 204.3 & 0.77 & 139.6 & 0.76 & 193.7 & 1.38 & 89.9 & 1.36 \\
\hline 133.9 & 0.39 & 240.6 & 0.77 & 339.1 & 1.37 & -14.0 & -0.03 & -24.3 & -0.01 & -37.4 & -0.03 \\
\hline 129.1 & 0.39 & 234.3 & 0.77 & 337.7 & 1.37 & -155.6 & -0.83 & -212.1 & -1.40 & -152.8 & -1.43 \\
\hline & & 0.9 & -0.01 & 328.7 & 1.37 & -254.0 & -1.43 & -314.9 & -2.20 & -289.3 & -3.03 \\
\hline & & & & & & -384.7 & -2.23 & -413.4 & -3.01 & -366.9 & -4.03 \\
\hline & & & & & & -224.3 & -1.43 & -278.9 & -2.20 & -234.5 & -3.03 \\
\hline & & & & & & -112.7 & -0.83 & -158.1 & -1.40 & -72.1 & -1.43 \\
\hline & & & & & & 35.7 & -0.03 & 45.4 & -0.01 & 48.5 & -0.03 \\
\hline & & & & & & 420.3 & 2.16 & 243.7 & 1.38 & 385.9 & 3.96 \\
\hline & & & & & & 417.9 & 2.16 & 450.8 & 2.98 & 383.4 & 3.96 \\
\hline & & & & & & 406.9 & 2.16 & 448.5 & 2.98 & 371.5 & 3.96 \\
\hline & & & & & & 0.9 & 0.01 & 437.5 & 2.97 & 1.0 & 0.37 \\
\hline
\end{tabular}

Tabelle A9: Zyklischer Belastungsablauf W7 